

\section{Micro-Macro and Rheology in sheared Granular Matter}

Abhinendra Singh 


\section{Samenstelling promotiecommissie :}

Prof. dr. Geert P.M.R. Dewulf (voorzitter) Universiteit Twente

Prof. dr. rer.-nat. Stefan Luding (promotor) Universiteit Twente

Dr. Vanessa Magnanimo (assistant promotor) Universiteit Twente

Prof. dr. Wolfgang Losert University of Maryland

Prof. dr. Devaraj van der Meer Universiteit Twente

Prof. dr. ir. Ton van den Boogaard Universiteit Twente

Dr. Brian Tighe Technische Universiteit Delft

Dr. Pierre Jop CNRS/Saint-Gobain, Paris, Frankrijk

Dr. Kuniyasu Saitoh Universiteit Twente

The work in this thesis was carried out at the Multi Scale Mechanics (MSM) group of the Faculty of Science and Technology of the University of Twente. This work is part of the research programme of the Foundation for Fundamental Research on Matter (FOM), which is financially supported by the Netherlands Organisation for Scientific Research (NWO).

Nederlandse titel :

Micro-macro en rheologie in granulaire materie

Cover design : Abhinendra Singh, Nishant Kumar (Software) and Rohit Shrivastava (Design and Implementation)

The background image is downloaded from http ://goo.gl/bf5nNH.

Publisher :

Abhinendra Singh, Multi Scale Mechanics, University of Twente, P.O. Box 217, 7500 AE Enschede, The Netherlands

Web : http: //www . utwente.nl/ctw/msm/

Email : a.singh-1@utwente.nl

Printer : Gildeprint, Enschede

(c) Abhinendra Singh, Enschede, The Netherlands, 2014

No part of this work may be reproduced by print, photocopy or any other means without the permission in writing from the publisher

ISBN : 978-90-365-3678-3

DOI : $10.3990 / 1.9789036536783$ 


\title{
MICRO-MACRO AND RHEOLOGY \\ IN SHEARED GRANULAR MATTER
}

\author{
PROEFSCHRIFT
}

ter verkrijging van

de graad van doctor aan de Universiteit Twente,

op gezag van de rector magnificus,

prof. dr. H. Brinksma,

volgens besluit van het College voor Promoties

in het openbaar te verdedigen

op vrijdag 14 mei 2014 om 16.45 uur

door

Abhinendra SINGH

geboren op 05 september 1983

te Dholpur, India 
Dit proefschrift is goedgekeurd door de promotor:

Prof. dr. rer.-nat. Stefan Luding

en de assistent-promotor:

Dr. Vanessa Magnanimo 
In the memory of my loving late Nanaji...

who made me, what I am today. 



\title{
Summary
}

\author{
Micro-Macro and Rheology \\ in sheared Granular Matter \\ by A. Singh
}

As a kid

I walked on the sand

but never sank in

My house stood on the ground,

but did not cave in

Made me think

soil is solid!

Growing older one day,

I read

about the Leaning Tower of Pisa

and I saw

a horrible landslide

The same soil

That lies beneath my foot

looked different than before

remains a mystery

is it solid or

is it liquid?

Soil, which is made up of countless interacting grains is a perfect example of granular material. The shape of the grains, the way they interact through contact, and the presence of humidity between them are all crucial to predict whether the soil beneath my house can withstand the load or if it would fail. When granular materials are sheared, the relative motion (flow) is confined to narrow regions (between large solid-like parts) called flowing zones. 
In the past couple of decades computer simulations, especially the Discrete Element Method (DEM) have evolved to become important tools to study granular matter. In this thesis, DEM simulations are used to study granular material in the critical state, by focusing inside the flowing zones induced by a special geometry called the split-bottom geometry. The aim of this work is to link the microscopic properties to the macroscopic bulk behavior as observed in experiments.

The thesis begins with the study of pairwise collisions between two elasto-plastic cohesive particles. A contact model, which takes all essential effects into account is introduced. With increasing impact velocity, a stick-rebound-stick-rebound behavior is observed. The first sticking range originates from the short-range non-contact attractive forces, while the second one appears due to the plasticity induced cohesion and dissipation.

Among the material properties influence on the macro-flow behavior, first focus is on the contact friction. Both the shear resistance of the material and the deviatoric fabric (structural anisotropy) first increase and then saturate with increasing friction, while the contact number density decreases. Increasing friction also increases heterogeneity in the spatial distribution of both the normal and tangential force network.

Next, a further level of complexity, cohesion is introduced. To determine the intensity of cohesive forces, a non-dimensional parameter called Bond number $B o$, which compares attractive forces to external compression forces is defined. $B o \approx 1$ captures the crossover from essentially noncohesive free-flowing granular assemblies $B o<1$ to cohesive ones $B o>1$. Various macroscopic and micro-structural features like the width of flowing zones and tails of force probability distributions are almost independent of cohesion for low Bond number,i.e., $B o<1$. Whereas, they get wider with increasing cohesion for high Bond number Bo $>1$.

As a next step, the effect of particle softness and gravity in the system are studied. So far in literature, the bulk behavior has been assumed to be independent of both. However our analysis, shows that the shear resistance of the material decreases systematically with increase in either softness or gravity. On the other hand, the shear resistance can be described as a unique power law, when analyzed against a non-dimensional number, which is the ratio of time scales related to softness and gravity. The structural anisotropy (deviatoric fabric) also shows a very similar behavior, that leads to an interesting interpretation that the shear resistance accompanies the anisotropy in the steady state contact network.

Finally, we look at the rheology of granular flows : simply put, how does the response of the system depend on the rate of shear. For low rates of deformation, the system is found to be an in almost rate independent regime. As the rotation rate is increased above a particular driving rate, the system enters a rate dependent regime. Both local shear resistance and structural (contact) anisotropy increase with increasing local strain rate. This shows that the shear resistance increases with strain rate mainly due to an increase in structural ani- 
sotropy, which indicates that the mesoscopic contact network dominates the behavior even for fast rate dependent flows, before the system enters the collisional regime for even faster strain-rate.

Using different tones composed in this thesis, a unique symphony can be orchestrated, which describes the flow behavior of soil on the Earth, as well as, on the Moon. In the end, the knowledge I gained increased my curiosity and at the end I have few answers but more questions than before. 


\section{Samenvatting}

\section{Micro-macro en reologie}

\section{in granulaire materie}

door A. Singh

Toen ik nog een kind was

Liep ik over het zand

Maar ik zakte er nooit in weg

Mijn huis stond op de grond

Maar het storte niet in

Did deek me denken

Grond is een vaste stof!

AMaar toen ik ouder werd

Hoorde ik

over de scheef staande toren van Pisa

en zag ik

Een verschrikkelijke aardverschuiving

ADezelfde grond

De grond waar ik op sta

Hij ziet er anders uit dan eerst

Het blijft een raadsel

Is het een vaste stof

of zoch vloeibaar?

Grond is een mooi voorbeeld van een granulaire materie, deze bestaat namelijk uit ontelbaar veel kleine korrels die onderlinge interactie met elkaar hebben. De vorm van deze korrels, de wijze van interactie en de mogelijke aanwezigheid van vloeistof zijn alleen cruciaal om het gedrag van dit soort materie te voorspellen. Dit samenspel maakt het moeilijk te voorspellen of mijn huis zal blijven staan of zal worden meegesleepd met een aardverschuiving. Als granulaire materie wordt afgeschoven, zal slechts een klein deel van de korrels bewegen, 
terwijl de meeste korrles op ongeveer dezelfde locatie zullen blijven liggen. Het gebied van de bewegende korrels noemen we ook wel het stromings gebied.

In de afgelopen decennia hebben comptersimulaties zich ontwikkeld tot belangrijke onderzoek instrumenten. In dit proefschrift wordt de Discrete Elementen Methode (DEM) gebruikt om granulaire materie in de kritieke toestand te simuleren. Met behulp van een "split-bottom"geometrie worden stromings gebieden gecreeerd, waarin de materie zich in de kritieke toestand bevindt. Het doel van dit onderzoek is een link te leggen tussen de microscopische eigenschappen en het macroscopische gedrag dat wordt gezien in vele experimenten.

Dit proefschrift begint met een gedetailleerde studie naar de paarse wijze botsing van twee deeltjes. Een eenvoudig contact model, dat toch alle essentiele effecten in ogenschouw neemt wordt ge $\tilde{A}^{-}$ntroduceerd. Met toenemende botsings-snelheden wordt een "stick-reboundstick-rebound"gedrag waargenomen. De eerste "stick"fase komt door de attractive krachten, terwijl de tweede "stick"fase komt door de cohesie en dissipatie die wordt vergroot door de plasticiteit.

Verderop in de proefschrift wordt het effect van deeltjes en systeem eigenschappen op het macroscopische bulk gedrag bestudeerd. Het doel is om de effecten van elke eigenschap te isoleren, zodat een duidelijk begrip van het complete elasto-plastische, wrijvingsvolle, cohesieve granulaire materie onder afschuiving wordt verkregen.

Als eerste wordt de aandacht gericht op het effect van wrijving. Zowel de afschuif weerstand van het materiaal als de structurele anisotropy, neemen initieel toe met toenemende wrijving, maar verzadigen later. Terwijl de contact dichtheid juist afneemt. Bij toenemende wrijving worden de "spatial distrubution"van zowel de normale als tangentiele krachten netwerken meer heterogeen.

In het tweede deel wordt de complexiteit verder verhoogd door cohesie te introduceren. Om de intensiteit van de cohesive krachten te kwantificeren word een de dimensieloze parameter, het Bond getal geintroduceerd. Dit getal vergelijkt de attractieve cohesieve krachten met de zwaartekrachten. Bo laat duidelijk de overgang zien van bijna niet chohesieve vrij stroomende granulare materie $B o<1$ tot cohesieve materie op $B o>1$. Verschillende macroscopische gedragingen, zoals de breedte van de stromings zones, zijn onafhankelijk van $B o$ voor $B o<1$, terwijl deze toenemen met $B o$ voor $B o>1$. "Micro-structural signatures" zoals de staarten van de kans dichtheid van de grote van de krachten laten een soortgelijke overgang zien.

Om nog een stap verder te gaan, worden de effecten van stijfheid en zwaartekracht bestudeerd. In de literatuur wordt tot heden verondersteld dat het macroscopische gedrag onafhankelijk is van beide. Onze analyse laat zien dat de afschuif weerstand van het materiaal systematisch afneemt met een toename zwaartekracht of afnemende stijfheid. De afshuif- 
weerstand kan beschreven worden met een unieke machtswet, wanneer deze geanalyseerd wordt als functie van de verhouding tussen de tijdsschalen geassocieerd met stijfheid en zwaartekracht. De structurele anisotropy laat een vergelijkbaar gedrag zien, wat leidt tot de interessante interpretatie dat de afschuif weerstand de anisotropie vergezeld in het contact netwerk.

Als laatste kijken we naar de rheology van dit soort stromingen. Simpel gezegd kijken we hoe het systeem reageert afhankelijk van de afschuifsnelheid. For langzame deformatie bevindt het systeem zich in een bijna snelheids onafhankelijk gebied. Wanneer de afschuifsnelheid verhoogd wordt, boven een bepaalde snelheid, wordt een snelheids afhangelijk gebied bereikt. Zowel de locale afschuif weerstand en de structurele anisotropy nemen toe met toenemende locale afschuifsnelheid. Dit laat zien dat de afschuif weerstand toeneemt met afschuifsnelehid voornamelijk door een toename in de structurele anistorpie. Dit geeft de indicate dat het mesoscopische contact netwerk het stromings gedrag domineert, zelfs voor snellere snelheids afhankelijke stromingen.

Uit de verschillende klanken in dit proefschrift kan een unieke symfonie gecomponeerd worden die het stromingsgedrag van grond beschrijft, op aarde zowel als op de maan. Uiteindelijk heeft de kennis die ik heb opgedaan mijn nieuwsgierigheid vergroot, en bij dit einde heb $i k$ weinig antwoorden maar meer vragen dan in het begin. 


\section{Acknowledgements}

I arrived in Enschede on 23 August 2009 and back then, I did not have a background in Granular Materials. However, today when I am writing the final chapter of my thesis, I feel content, happy and confident that I took the right decision. Regarding my interest towards research, I owe a big thanks to Dr. Ram Ramaswamy, who taught me that learning science can be fun too. The long conversations about research over coffee with him inspired me to think about a career in science. Dr. Rama Govindarajan plays an equally important role as she introduced me to the world of numerical research. Heartfelt thanks to $D r$. $G$. P. Raja Sekhar, for being my master thesis supervisor against all odds and helping me with the applications for Ph.D.

The memory goes back to the day of 9 March 2009 when I sent an email to Prof. Stefan Luding with the subject "Request for PhD position in your group". Following through the process of a Skype interview and sending in the recommendations, finally in June 2009 Stefan offered me a position. I was thrilled reading the email which stated that "You are the No. 1 on our list of candidates". After a master in Physics and my inclination towards research, I grabbed this opportunity without hesitation.

First of all I would like to thank Stefan, for accepting me to be a part of his group and introducing me to the research in granular materials. The very first thing that comes to my mind when I think about Stefan is that he is simply awesome as a supervisor. He gave me all the freedom to design my thesis. As a supervisor, he has always been available, patient, motivating, and supporting in all aspects. He has always shown keen interest towards all my questions and discussion, some of which have even lasted for 3-4 hours. Those fruitful discussions have helped me grow as a researcher. I absolutely adore his effective and efficient, yet cool attitude. I have always wondered so as to how does he manage to switch among various topics of discussions, yet remained focused always. Understanding my personal life, you even allowed me to visit Singapore bridging a talk in China so that I could spend some time with my newly wedded wife. Stefan, I have learnt a lot from you not only about Science, but also about how to be a nice person. I would also like to thank Gerlinde for being so warm and nice to me all the time. I still remember her super happy 
face when I told her about my marriage. She has always been very kind and has always been concerned about the welfare of my family. Thank you very much!

Vanessa, my co-advisor also deserves a big Thank You. Undoubtedly, she is the best person as a daily supervisor. Her insight from an engineering point has broadened my vision and approach in dealing with the problem. She was always been enthusiastic about new results and for the long discussions thereafter. She cannot be convinced easily which in turn has helped me to strengthen my concepts. Long lasting discussions with her about work, be it rheology, cohesion or friction were simply remarkable and intriguing. Her efforts in reading my papers and criticizing them made me improve my way to interpret the scientific data. This in turn helped me to present them in a clear and concise manner without which this thesis would have never seen the daylight!

The phenomenal collaboration with Kuni in the final year has been of significant help to me. I wish if it were started much before. You have noticeably helped me a lot in winding up my research in the final phase. I truly appreciate your feedback, corrections and enthusiastic discussions for the best. I have profited a lot from your insight in physics.

Sylvia has been my mom in The Netherlands. I have troubled you a lot, but you always welcomed me with arms wide open and a smiling face. When no one could solve it, my mom had a solution to all the questions and problems I faced. You had been the anchor for me and with you I never missed being away from home. Thanks a lot for talking to me about all my stupid personal/professional issues I ever had. Thanks to you and your family for showering so much of love everytime we met.

In no particular order, I am thankful to all my past and present colleagues of Multi Scale Mechanics group - Sebastian, Fatih, Vitaliy, Saurabh, Nico, Rohit, Sudeshna, Dinant, Wouter den Otter, Anthony, Thomas, Kazem, Nishant, Kay, Mateusz, Ibrahim, Lisa, Stefan, Deepak, Martin, Aurelien, Fabian. A noteworthy thanks to Dinant for helping me with the translation of the summary into Dutch. I thank Wouter den Breeijen, for helping me with the technical issues of system and also for giving me extra space on the Cluster. I enjoyed the interesting discussions with Fatih, which I truly missed in my final year. I embrace the special friendship with Mateusz who very enthusiastically wrote many scripts without which the data analysis would have been very difficult. Aurelien, thanks very much for all the wonderful discussions we had regardging religion, society, and science. Special thanks to Rohit and Nishant for working day and night on the cover page of this thesis. Rohit, you should have joined MSM earlier, may be a year before.

I would like to thank all the committee members for their interest in my research. I am grateful to Prof. Wolfgang Losert for taking the pains to arrive here all the way from US. Dr. Pierre Jop, thank you for appreciating my work and taking out time for the Skype conversation. I am thankful to Dr. Brian P. Tighe for immediately accepting the invitation for my defense. I feel honoured that Prof. Devaraj showed keen interest in my work since the very 
beginning. Prof. Ton, thank you for making me comfortable in the 2-hour long discussion over my thesis. I have thoroughly enjoyed every bit of discussion with all my collaboratorsMario Liu, Matthias Schröter, Shuiqing Li, Dirk and Reza. Zhu and Mengmeng Yang, thanks for making our trip to Beijing memorable. We will truly cherish all the moments.

Nishant is my dear friend whom I could trust and rely on at all times. You have always stood by me, when I was high, low, sober, drunk, exhausted, and excited. I appreciate your constant push so as to work hard and with sincerity. The quite yet active friendship of Shushil is something that I will cherish forever. Your willingness to help has always made me feel special. I learnt a lot from you. Ankit Verma, I have always enjoyed your company which comes as a surprise to me even. Our bonding grew with time and I am glad that we share such companionship. Thank you all three for the great and lovely times playing 29, badminton and tennis. Ankit Jain, thanks for cheering me up at all times. Thanks to Megha and Pradeep for visiting us.

I am blessed to have Pallavi and Reddy as a family away from home. Both of them are truly a gem of a person. The relationship that we share is beyond formality, and all the credit goes to the love and care that you have showered on me. Pallavi, just a thought of the delicious dosas that you cooked for me still makes my mouth watering. Thank you Reddy for the teachings, and yet being so cool and calm. On top of all, thank you for the most beautiful gift, Aadya. Thank you for introducing us to Arun Bhaiyya and Suryakanthi Babhi. Their hospitality was truly heart-warming. Suryaansh, you are a genius.

The small get-togethers with Sampada and Omkar were really enjoyable. I am grateful to Omkar for taking time out from his busy schedule and providing me with feedback comments on my piece of work. Amogh, you are special as you are the first one to call me Kaka! I have earned a few more friends on the tennis court. Hammad, I appreciate your warm and humble attitude at all times. Urooj, I am big fan of your cooking skills. Special thanks to - Neha-Saurabh, Chaithanya-Shodhan, Neeru-Hanumant, Neelam-Jitendra, MeenakshiChandu, Anne-Ravi, Antina-Vijaya, Zed, Stephan Ulrich, Arpita, Kartikeya, Naveen, Vivek.

Tasja, Kaman, PG, Phan Phi and Li were my first ever friends in the new country. You all made my stay in Netherlands comfortable and enjoyable. I owe a lot of great memories to you all. I am lucky to have friends like Ashish Goel, Breeta and Pretty who took time off and did the last minute revisions. A special mention of all my dear friends from India - Resham, Mohit, Anchal, Neeraj, Devanshu, Piyush, Tarun, Gopal, Gaurav, Rohit Omar and Praveen. Ashish Malik, your special efforts in travelling from Nancy to Paris just to meet me are truly appreciated.

Finally, I would like to thank my family. I am obliged to Maa and Papa for making me what I am today. Without your love, care and support all this would not have been possible. I know maa you would have had sleepless nights without me, but all your sacrifices have now finally paid off. I am grateful to my grandparents for the blessings they bestowed upon me. I would 
also like to thank my sisters and their husbands, Alka-Abhishek and Priyanka-Abhitesh, for their love and the belief they had in me. I love you my dear nephew Atharva, and my niece Ananya. A thank to all my cousins, uncles and aunts. The constant support and motivation of my in-laws has been very special. I truly appreciate the efforts of my brother-in-law, Varun who always took out time in the midst of his exams and submissions for editing the summary and conclusion. Last but not the least, my wife Shruti deserves a pat on her back for bearing with me in my tough times and listening to me when I was frustrated/irritated. Shruti, thanks a lot for all your sacrifices, your prayers and your invaluable support.

To everyone, who contributed and has been a part and parcel of my life. My sincere apologies if I missed to mention anyone. You mean no less to me, and I appreciate you all.

Abhinendra Singh Enschede, April 2014 


\section{Contents}

Summary $\quad$ v

$\begin{array}{ll}\text { Samenvatting } & \text { ix }\end{array}$

Acknowledgements $\quad$ xiii

1 Introduction 1

1.1 Granular Materials . . . . . . . . . . . . . . . . . . . . . . 1

1.2 Goal . . . . . . . . . . . . . . . . . . . 3

1.3 Story of the thesis . . . . . . . . . . . . . . . . 4

2 Granular Flow Review $\quad 7$

2.1 Slow Flows . . . . . . . . . . . . . . . . . . . 7

2.2 Fast Flows . . . . . . . . . . . . . . . . . . . . . . 15

2.3 Methodology ........................... 16

3 Contact model for sticking of adhesive mesoscopic particles 19

3.1 Introduction . . . . . . . . . . . . . . . . . . . . . . 21

3.2 Discrete Element Method . . . . . . . . . . . . . . . . . . 26

3.3 Coefficient of Restitution . . . . . . . . . . . . . . . . . 38

3.4 Elasto-plastic coefficient of restitution . . . . . . . . . . . . . 42

3.5 Conclusions . . . . . . . . . . . . . . . . . 52

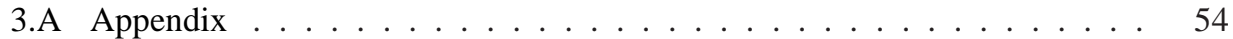

3.B Energy Picture . . . . . . . . . . . . . . . . . . 59

3.C Tuning of parameters to increase the plastic range . . . . . . . . . . 61

3.D Agglomerate compression and tension test . . . . . . . . . . . 63

4 Effect of friction and cohesion on behavior of granular materials 67

4.1 Introduction . . . . . . . . . . . . . . . . . . . 68

4.2 Model System Geometry . . . . . . . . . . . . . . . . . . . . . . . . . 68

4.3 Results . . . . . . . . . . . . . . . . . . . . 70

4.4 Discussion . . . . . . . . . . . . . . . . . . 80 
5 Effect of cohesion on shear banding in granular materials 83

5.1 Introduction and Background . . . . . . . . . . . . . . . . . . . . . . 84

5.2 Discrete element method simulation $(\mathrm{DEM}) \ldots \ldots \ldots$. . . . . . . 85

5.3 Results . . . . . . . . . . . . . . . . . . . . . . 87

5.4 Discussion and conclusion . . . . . . . . . . . . . . . 101

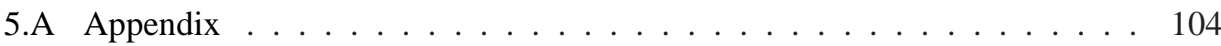

6 DEM simulations of granular rheology: Effects of gravity and contact stiffness.105

6.1 Introduction . . . . . . . . . . . . . . . . . . . . . 106

6.2 Discrete Element Method . . . . . . . . . . . . . . . . . . . . . . . . 107

6.3 Quasistatic state ....................... . 110

6.4 Dense inertial regime . . . . . . . . . . . . . . . . . . . . . . . 119

6.5 Discussion and Conclusion . . . . . . . . . . . . . . . . . 123

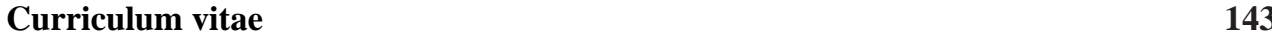




\section{Chapter 1}

\section{Introduction}

Matter is usually classified into solids, liquids, and gases. But what about granular matter? Dry sand flows in a hourglass. When poured into a container, it adapts to the shape of the container displaying a property of liquids, while at rest it appears to be "solid". At the level of a single grain of course it is a solid, but collections of a lot of grains together are granular material, with quite different properties.

Dune migration, landslides, avalanches, and silo instability are a few examples of systems where granular materials play an important role. Furthermore handling, and transport of these granular materials are central to many industries such as pharmaceutical, agricultural, mining and construction industries and pose many open questions to the researchers.

\subsection{Granular Materials}

In spite of the ubiquity of granular systems, understanding their behavior is a major challenge for science. Even in a seemingly simple system such as dry sand, the presence of large numbers of internal degrees of freedom lead to highly nonlinear effects, which makes it difficult to relate the microscopic grain level properties (known) to the macroscopic bulk behavior.

\section{Basic properties -}

In cases of misfortune when an earthquake hits, our home or office begins to vibrate. Alas, it is too late to think about the strength of the ground under our feet, because in many cases soil does not act as you expected. Normally it is a solid, but when it is fluidized the liquid like behavior of soil leads to destruction. Hence it is important to understand how and when soil 
flows. Lucretius (ca. 98 - 55 B.C.) was probably the first one to recognize this interesting behavior of soil-like materials, when he wrote "One can scoop up poppy seeds with a ladle as easily as if they were water and, when dipping the ladle, the seeds flow in a continuous stream "(text taken from Duran [45]).

This complex macroscopic behavior has many origins. First, a granular constituent is much larger than atoms and molecules composing it, this makes it insensitive to thermal fluctuations. The gravitational energy $m g d$ of a $1 \mathrm{~mm}$ sized sand grain and kinetic energy acquired by it (when raised by its own diameter) exceed the thermal energy $k_{B} T$ by many orders of magnitude $[37,72]$. Second, granular interactions are dissipative in nature. This means, kinetic energy is lost during collision due to inelasticity and friction at contact. This property distinguishes granular materials from ordinary liquid or gases, where the energy is conserved during collision between atoms or molecules. The athermal and dissipative nature of interactions lead to a system far away from equilibrium. Dissipation and irrelevance of temperature are primary reasons of difficulties faced while explaining granular materials using theories like thermodynamics and statistical physics.

Granular solid, liquid or gas? - An interesting feature of granular materials is the fact that they can behave as solids, liquids, or gases, depending on the way the material is driven $[55,72]$. Fig. 1.1 shows a typical flow obtained by pouring steel beads on a pile. Three distinct phases can be clearly observed: on the top is a dilute regime where the beads bounce in all directions, and collisions are the dominant interaction between them. This regime is referred as gaseous regime, and will not be touched in this thesis; interested readers should refer to [57] and references therein. Just below this gaseous regime, a semi-dilute phase exists, where the beads have enduring frictional contacts, but still flow past each other. Below this liquid phase, deep into the bulk of the heap is the solid phase, where the particles do not have much free space to move. In this phase, particles are almost static, they do not experience collisions, but have enduring contacts.

The coexistence of these diverse phases makes the behavior of granular materials rather complex, which is hard to be captured by a unique model. Given the wide presence and applications, a model which describes broad, general concepts that can explain all collective systems is particularly appealing to physicists, as well as mechanical and civil engineers.

A particular area of interest of many scientists is the flowing behavior of granular materials under shear, due to its application in geophysics for description and prediction of natural risks such as landslides, avalanches etc. The flowing behavior of granular materials is remarkably different from what one would expect from Newtonian fluids. When granular materials are sheared, the shear is not distributed homogeneously throughout the system, instead it gets localized to narrow regions called shear bands. In other words, only narrow regions between the large solid-like parts show flowing behavior.

Another field of interest in the granular community is 'Jamming', where granular materi- 


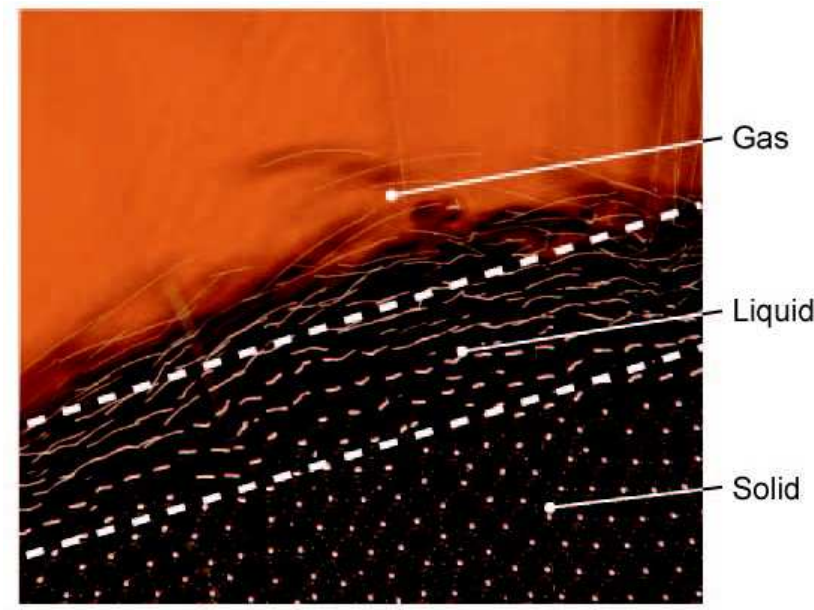

Figure 1.1: An image of steel beads poured on a pile illustrates the three distinct phases of granular material. Adapted with permission from [55]

als above a critical packing fraction (jamming point) are found to be mechanically stable with finite stiffness $[17,96,135,137,145,175,185,213,233]$. The belief in a jamming "point"was recently questioned by [17, 32, 134, 234].

\subsection{Goal}

To begin with the goal of this thesis, I would like you to consider a jar filled with sand grains. The sand grains behave like a solid, supporting the weight of the particles above. When the jar is tilted gently about an axis, above a critical angle, called the angle of repose, sand begins to move/flow. When one looks closely, the topmost layers flow like a liquid, while the bottom part is still solid: a shear band forms at the interface of the two. This is the simplest small-scale analog of what happens in natural large-scale granular flows like avalanches or land slides.

The aim of this thesis is to study the boundary between the liquid and solid phases by studying granular flows. How do the microscopic material and system parameters influence the macroscopic flow behavior of the bulk system? This question remains paradigmatic and will be addressed in the thesis. Citing the above example of sand grains in a jar, the onset of flow, i.e., the angle of repose would depend on many parameters. If one fills two separate jars with the same amount of rough and smooth particles, from intuition one can say that jar with rough sand will have higher a angle of repose. But then few questions emerge: how does the bulk macroscopic angle of repose change with microscopic roughness of the particles? Does the jar filled up of purely smooth (frictionless) grains have a zero angle of repose? Activating 


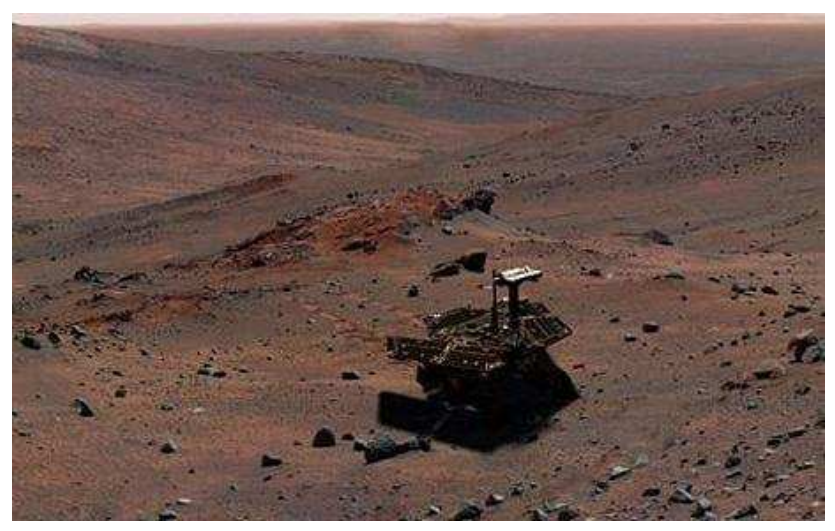

Figure 1.2: A synthetic image of the Spirit Mars Exploration Rover: Mars rover stuck in soil. Figure adopted from Ref. [55].

attractive forces at contact e.g. by simply adding some liquid even further complicates the picture.

A new question which becomes important for scientists interested in the geology of planets is, whether the external compression affects the flow behavior of the granular materials? In other words, can one assume the flow properties, on Moon or Mars to be the same as that found on Earth? Or does the soil found deep down Earth's surface have the same properties as soil found on Earth's surface? A wrong estimate of the failure property of soil can be dangerous, as shown in Fig. 1.2, which shows the Mars rover stuck in soil.

This thesis tries to answer the questions raised in this section, by focusing on how given, known micro-mechanical properties affect the unknown macroscopic continuum behavior of the bulk granular material.

\subsection{Story of the thesis}

To understand the flow behavior of granular material at the solid-liquid interface, we perform numerical simulations in the split-bottom geometry [54]. The focus of this thesis is to study the effect of material and system parameters on the bulk behavior of granular material.

A brief review of granular flows in various commonly found geometries is presented in Chapter two. We begin with a review of slow granular flows, where enduring contacts are dominant. Since Split-bottom cell is the geometry used throughout the thesis, the major works done in this geometry are briefly discussed. In later part of this chapter, fast granular flows are also discussed.

Interaction laws between the particles are at the heart of DEM simulations. To begin with, 
in Chapter three pairwise contacts and collisions between meso-particles are studied. A brief review of cohesive, elasto-visco-plastic contact models is presented. Using energy conservation arguments, the dependence of the coefficient of restitution on impact velocity is studied. A new sticking regime is observed, which is induced by a balance between nonlinear, history dependent cohesion and plastic dissipation.

The rest of the thesis deals with the flow behavior of granular matter under quasi-static shear in a split bottom ring shear cell, while in the last chapter both slow and fast flows are studied. The effect of particle friction and cohesion on steady state anisotropy is the focus of Chapter four. For noncohesive granular material, macroscopic friction and fabric anisotropy are found to behave similarly. Both are found to saturate after an initial increase with increasing contact friction, with the major contribution coming from the strong contact network. We analyze the probability distribution functions (PDFs) of both normal and tangential forces. For cohesive powders, shear stress becomes nonlinearly dependent on confining pressure. The contact network is found to be more isotropic for system with higher cohesion. This observation suggests that with changing cohesion, the contacts along compressive and tensile directions rearrange, such that total number of contacts stay the same.

Chapter five, deals with the effect of contact cohesion on slowly sheared dense, dry, frictionalcohesive powders. We study the effect of cohesion on the normal force network and velocity profiles in the steady state. A dimensionless number granular Bond number $(\mathrm{Bo})$ is used to estimate the strength of attractive cohesive forces. The mean force inside a shear band is independent of cohesion, while the heterogeneity and anisotropy of the force network are found to increase with cohesion. Bo $=1$ is found to be a control parameter for the shear banding phenomenon, which undergoes a transition from being cohesion independent for $B o<1$ to cohesion dependent for $B o>1$. The explanation for this transition is presented in this chapter.

For a long time, the macroscopic friction coefficient for a given material has been assumed to be independent of magnitude of gravity. Chapter six aims to test this assumption by studying frictional granular matter under slow shear with gravity varying over two orders of magnitude. The macroscopic friction coefficient is found to monotonically decrease with increasing gravity. A collapse of the data is observed on a unique curve when the ratio between forces due to gravity and contact stiffness is used as a scaling parameter. The contact anisotropy behaves in a similar way as the macroscopic friction, correlating with macroscopic friction. We further show that this correlation, which is found in slow granular flows can be further extended to dense inertial flows, but fails for rapid flows.

In chapter seven, the scope of the thesis broadens, and both fast and slow flows are studied. A three dimensional local rheology model is the focus of this chapter. Traditionally, extensive homogeneous volume or pressure conserving experiments have to be performed to study the 
critical state rheology. Here, from a single simulation a wide range in local strain rate, shear and normal stresses, and volume fractions can be extracted. In the steady state, the system is found to be heterogeneous, and the local rheology shows a transition from a quasistatic regime at low shear rate to an inertial regime, where the shear stress ratio increases with shear rate. The evolution of the microstructure of the material is well characterized by a suitable parametrization of the fabric tensor and the coordination number.

The thesis ends with conclusions and recommendations for future research. 


\title{
CHAPTER 2
}

\section{Granular Flow Review}

\begin{abstract}
We review flows of dense cohesionless granular materials, with a special focus on splitbottom geometries. We first discuss slow flows in basic and most common geometries, which is characterized by enduring contacts. Then a brief review of recent works on the flows in split-bottom geometry follows. Finally a description of fast flows is presented, where binary collisions are dominant mode of interaction. In the last section, methodology of the numerical technique used in this thesis is briefly introduced.
\end{abstract}

\subsection{Slow Flows}

The motion in assemblies of grains has to be first induced, in order to study granular flows. The flow can be achieved by imposing an external stress on the material, or by applying a shear to the material. In this chapter, the focus is on the dense liquid regime, which is most often encountered in applications. To begin with, only systems with dry grains and without any cohesive interactions are discussed here.

The work of illustrious scientist Coulomb, who first explained the yielding of granular material as a frictional process, laid the basics of slow granular flows. He was interested in prediction of soil failure for Civil Engineering applications. Few basic and most common geometries (Fig. 2.1), are discussed below:

2.1.0.0.1 Inclined plane One common flow geometry, the inclined plane is encountered in both geophysical and industrial contexts. The grains are poured from a large reservoir onto 


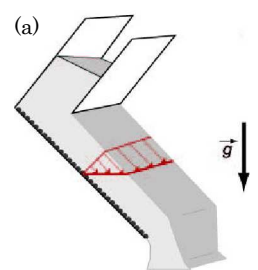

(c)

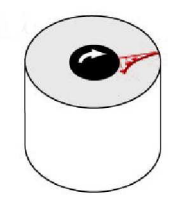

(b)

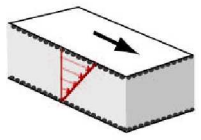

(d)

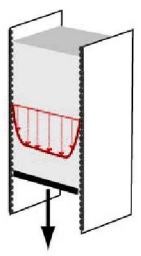

Figure 2.1: Four flow geometries (a) inclined plane; (b) plane shear; (c) Couette; (d) channel. Figures adapted from Ref. [55].

a chute plane placed at some defined angle with respect to gravity direction. The tilt angle of the plane, controls both the flow and stress acting between the particles. One interesting point is, lowering the tilt decreases the stress, while the resulting flow suddenly speed does suddenly drops to zero - below a certain threshold inclination, the flow stops; the packing jams.

2.1.0.0.2 Plane Shear Flows The plane shear geometry is one of the simplest ways to impose shear deformation. In this geometry, the material is sheared between two parallel plates; Numerically the stress distribution is found to be uniform inside the shear layer, however experimentally it is not achieved owing to the presence of gravity [118]. The most common method of inducing shear in this geometry is by imposing the wall velocity [10].

2.1.0.0.3 Couette Flows Couette flow is also classically referred as "Annular shear flow". This is one of the classical geometries used to study the flow behavior of complex fluids. In this geometry the material is sheared mainly due to relative motion between (concentric or conical) cylinders. In this geometry, shear is localized on a few particle layers close to the inner moving boundary $[91,118]$, which is robust in the sense that it exists independent of dimensionality and rotation rate $[91,123]$. The shear stress necessary to sustain the flow in most of the cases is independent of rate of rotation, though for some compressed systems a logarithmic dependence is found [61].

2.1.0.0.4 Channel Flows Vertical channel flow in principle is made up of two parallel walls filled up with material between them. The velocity profiles are reminiscent of a plug flow in the center part of the material, where velocity almost remains constant, hence the material is not sheared. Shear is localized in narrow shear bands close to the boundary with 
thickness of the order of 5-10 particle diameters [129]. Flow is found to be intermittent for some special cases [16], which can be associated with sudden appearance of load bearing force network configurations [150]. Jamming of particles at the orifice can also lead to complete arrest/blockage of the flow [160, 200], a problem which disappears for large enough orifice size.

One special and common property of the above mentioned setups is that the material undergoes dilation. This phenomenon was first observed by Osborne Reynolds [156], who named it dilatancy. He performed a rather simple experiment by filling a bag with water and grains, and observed that additional amount of grains can be added once the bag is deformed, i.e., the density of grains decreased upon shearing.

Another common feature of slow granular flows is localization of strain in shear bands of few particle diameters width. Shear bands have been studied extensively in geomechanics because of their role in natural hazards such as landslides and avalanches [41]. Capturing the width of a shear band with continuum models has been challenging because of the lack of a microscopic length scale reflecting the microstructure. As a result, micro-polar continuum models such as by Cosserat [48] have been put forward to regularize, i.e. get a finite width of the shear band.

Apart from the setups described above, another geometry proposed recently which allows one to impose an external deformation at constant rate is the split-bottom geometry [54]. In this geometry, stable shear bands of arbitrary width can be achieved allowing for a detailed study of microstructure within the shear band. Since the split-bottom geometry is the geometry studied in the whole thesis, a detailed description is given below.

\subsubsection{Split-Bottom Geometry}

In this section, a brief review of recent experimental, numerical and theoretical work on the flows in this geometry is presented.

\subsubsection{Description}

In the split-bottom geometry, the granular material is not sheared directly from the sidewalls, but from the bottom. The bottom of the setup that supports the weight of material above it is split in two parts, the two parts move relative to each other and creates a wide shear band away from sidewalls. The resulting shear band is robust, as the location of the shear band exhibits simple, mostly grain independent properties. This makes it a im-practicable device for measuring grain properties, but has advantages, as well be detailed and used in this thesis.

Two variants of the split-bottom geometries are popular: in experiments, cylindrical split- 


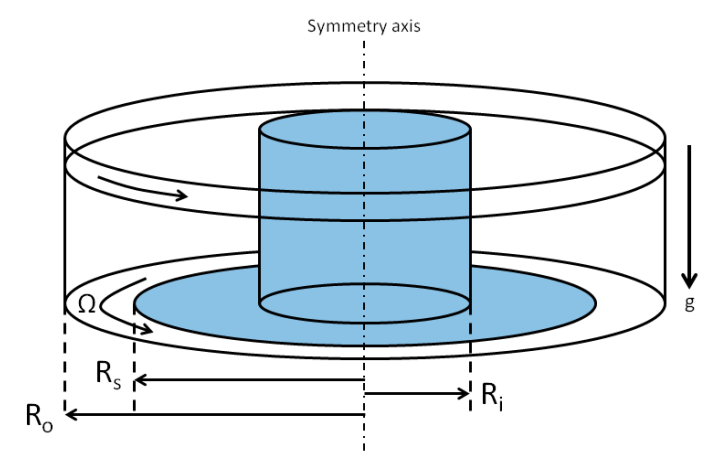

Figure 2.2: A sketch of our numerical setup consisting of a fixed inner part (light blue shade) and a rotating outer part (white). The white part of the base and the outer cylinder rotate with the same angular velocity $\Omega$ around the symmetry axis. The inner, split, and outer radii are given by $R_{i}=0.0147 \mathrm{~m}, R_{S}=0.085 \mathrm{~m}$, and $R_{o}=0.11 \mathrm{~m}$, respectively, where each radius is measured from the symmetry axis. The gravity $g$ points downwards as shown by arrow.

bottom shear cell is used, which is typically a Couette cell with a split at the bottom [31, 52$54,67]$, while a linear split-bottom cell is also used in some studies [38, 39, 159]. In this thesis, we use a cylindrical split-bottom shear cell, which is found to give good agreement with experiments [104].

Fig. 2.2 is a sketch of the cylindrical split-bottom shear cell used in this thesis. In this figure, the inner, split, and outer radii are given by $R_{i}, R_{S}$, and $R_{o}$, respectively, where the concentric cylinders rotate relative to each other around the symmetry axis (the dot-dashed line). The ring shaped split at the bottom separates the moving and static parts of the system, where a part of the bottom and the outer cylinder rotate at the same rate.

\subsubsection{Control Parameters}

The split-bottom geometry is characterized by three parameters: the split radius $R_{s}$, height of the granular layer $H$, and the rate of rotation $\Omega$ (of the outer cylinder and the base). The driving rate $\Omega$ is generally fixed in initial series of experiments, and the relative motion of the split with respect to the cylinder drives the flow. The thickness of granular layer $H$, is scanned in a series of experiments. Note that, the radius of the outer cylinder appears to be immaterial, if it is sufficiently large $[52,54]$. The interesting observation in the experiment is a universal shear zone, initiated at the bottom of the cell and becoming wider and moving inwards while propagating upwards in the system, as shown in Fig. 2.3.

The ratio of averaged azimuthal velocity of the grains, $v_{\theta} / r$ and external rate of rotation $\Omega$ is denoted by $\omega ; \omega=0$ signifies stationary grains, while $\omega=1$ corresponds to particles 
moving with the driving. The grains moving with $\omega$ between the two extremes correspond the flowing part, i.e. the shear band. Blue colored particles in Fig. 2.3 are practically static, red colored particles co-move with outer cylinder, while green colored particles denote the shear band.

\subsubsection{Shear deformation}

2.1.1.3.1 Shallow flows - We begin with the discussion of the flow profile observed at the free surface. As shown in Fig. 2.3, from the top view, it is evident that the shear band moves inwards with increasing filling height, and it also becomes wider without any upper bound [104].
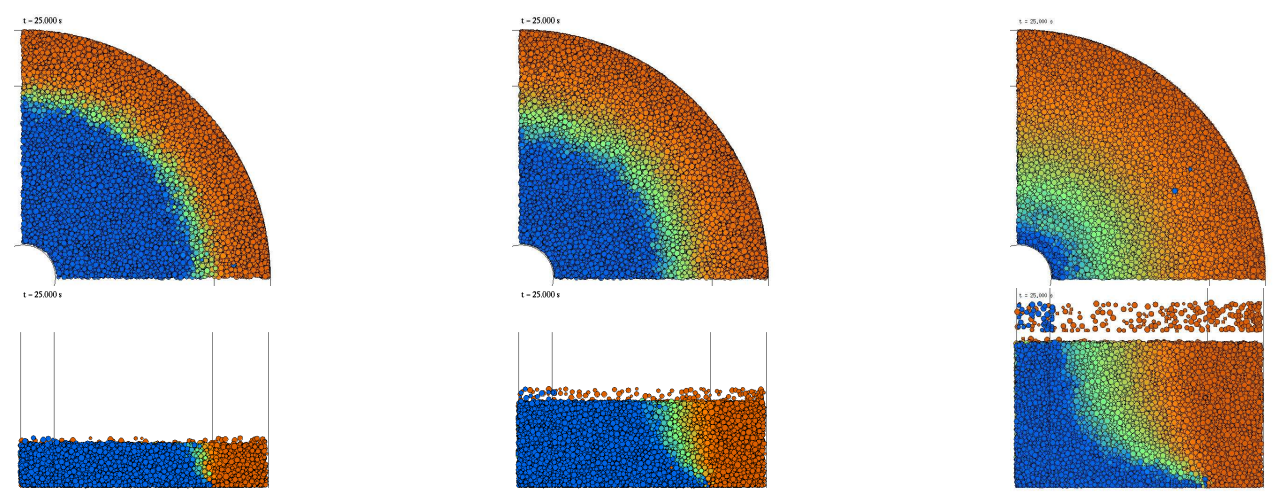

Figure 2.3: Snapshots from simulations with different filling heights seen from the top and from the front, and the number of particles being (Left) $N=16467$, (Middle) $N=34518$, and (Right) $N=60977$. The colors blue, green, orange and red denote particles with $r \mathrm{~d} \phi \leq$ $0.5 \mathrm{~mm}, r \mathrm{~d} \phi \leq 2 \mathrm{~mm}, r \mathrm{~d} \phi \leq 4 \mathrm{~mm}$, and $r \mathrm{~d} \phi>4 \mathrm{~mm}$, i.e. the displacement in tangential direction per second, respectively. The filling heights in these simulations are $H=0.018 \mathrm{~m}$, $0.037 \mathrm{~m}$, and $0.061 \mathrm{~m}$ (from left to right) Figure reprinted with permission from Ref. [104].

After proper rescaling, all bulk profiles collapse on a universal curve which can be extremely well fitted by

$$
\omega(r)=\frac{v_{\theta}(r)}{r \Omega_{o}}=A\left(1+\operatorname{erf}\left(\frac{r-R_{c}}{W}\right)\right),
$$

where erf denotes the error function, $r$ is the radial coordinate, $R_{c}$ the center position of the shear band (maxima of velocity gradient), and $W$ the width of the shear band. Accurate measurements of the tails of velocity rule out exponential tails, rather suggesting, that the strain rate is Gaussian-like, and the shear bands are completely determined by their centers $R_{c}$ and width $W$ [52]. Particle shape does not much influence the functional form of the velocity profiles, which contrasts the particle shape dependence found for wall-localized shear bands in Couette cell [123]. 
The center of the shear band is found to be independent of the material used [52]. Therefore, the relevant length scales for the position of shear band are $R_{S}$ and $H$. The fits to the velocity profile from simulations confirm this finding, and a simple relation

$$
R_{s}-R_{c} \propto H^{5 / 2}
$$

very well describes the behavior as shown in Fig. 2.4.

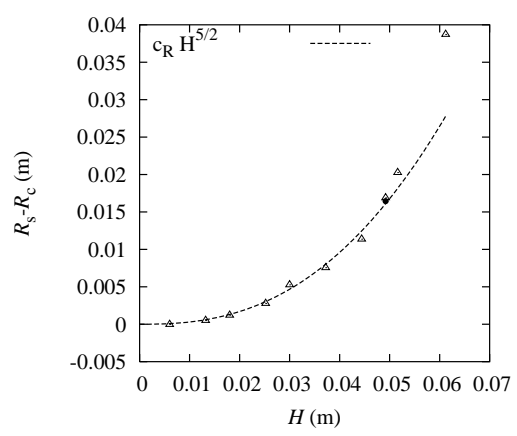

(a)

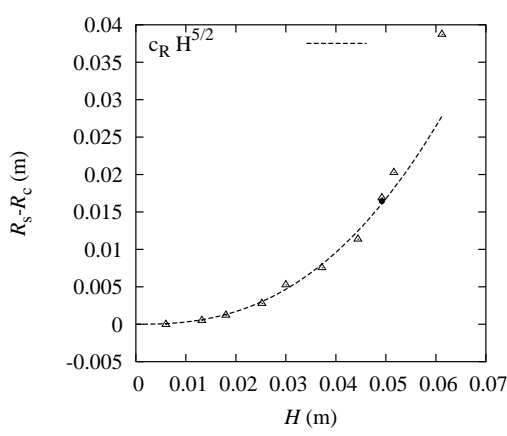

(b)

Figure 2.4: (Left) distance of the top-layer shearband center from the slit, both plotted against the filling height $H$. The open symbols are simulation results, the solid symbol is a simulation with slower rotation $f_{o}=0.005 \mathrm{~s}^{-1}$, and the line is a fit with constant $c_{R}=30$. (Right) width of the shearband from the same simulations; the line is a fit with $c_{W}=2 / 5$. Figure reprinted with permission from Ref. [104].

The width of the shear band $W$ depends on the grain properties, and is almost independent of $R_{S}$ [52]. Grain shape, size, and contact properties affect the width: spherical particles display wider shear bands compared to irregular ones of the same size. Rough particles display narrower shear bands compared to smooth particles [105]. Experimental data shows $W \sim$ $(H)^{2 / 3}$, while simulations show that the width of the shear band increases almost linearly with the filling height $W \sim H$, as shown in Fig. 2.4.

Experiments using colored beads [52] and MRI [31, 163], and numerical simulations [39, 103-105, 159] have shown that the flow profiles at fixed depth $h$ below the top surface $H$ can be expressed using Eq. 2.1. This allows to characterize $v_{\theta}(r)$ at a given $h$, the fits to simulations results help us to understand position and width of the shear band in the bulk. Very much like in the experiments, the behavior of the shear band within the bulk, see Figure 2.5 , deviates qualitatively from the behavior seen from the top. Instead of a slow motion of the shear band center inwards, the shear band rapidly moves inwards at small heights $h$, and reaches a saturation distance with small change closer to the surface. Again, a slower rotation does not affect the center but reduces the width. In the bulk, position of the shear band is very well predicted using variational principle by Unger et. al. [209]. Numerical 
study by Ries et. al. [159] showed that $W(h)$ can be described by the functional form as

$$
W(h)=W(H) \sqrt{1-(1-h / H)^{2}}
$$

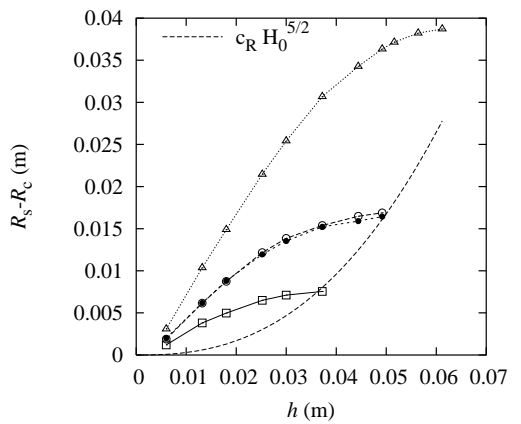

(a)

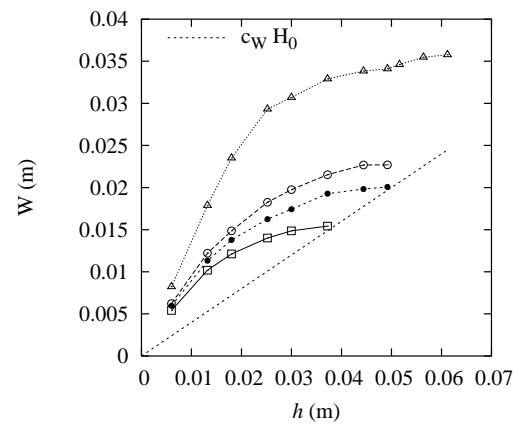

(b)

Figure 2.5: (Left) distance of the bulk shearband center from the slit. (Right) width of the shearband, both plotted against the height $h$. The open symbols are simulation results obtained with $f_{o}=0.01 \mathrm{~s}^{-1}$, the solid symbols are obtained with slower rotation $f_{o}=0.005 \mathrm{~s}^{-1}$. Squares, circles and triangles correspond to the filling heights $H=0.037 \mathrm{~m}, 0.049 \mathrm{~m}$, and $0.061 \mathrm{~m}$, respectively. The dashed curves are identical to those plotted in Fig. 2.4. Figure reprinted with permission from Ref. [104].

This thesis mainly focuses on moderately shallow flows in the split-bottom cell, below we give a brief overview on deep flows.

2.1.1.3.2 Deep flows When the ratio $H / R_{S}$ is small, the core material rests and moves together with the center disc. With increase in $H / R_{S}$, the shear band grows wider and moves inside. The most striking feature is that the core now precesses with a constant rate, hence material in the central part of the surface no longer rests on the disc. Precession is not simply the consequence of the overlap of two opposing shear zones, since before being eroded by shear, the inner core rotates as a solid blob for an appreciable time [53]. For various split radii, the onset of precession grows with $R_{s}$, while it is mainly controlled by the ratio $H / R_{s}$. For $H / R_{S}$ of order one, the whole surface rotates rigidly with the rotating drum, and shear is concentrated in the bulk. While, for $H / R_{S}<0.65$, hardly any precession is observed [53]. When $H / R_{S}$ is sufficiently large, the shear band is entirely confined to the bulk, and a dome-like structure is formed above the split [31, 53, 163, 209]. 


\subsubsection{Dilatancy}

The sheared granular material is known to dilate [156]. Sakaie et al. [163] presented results on evolution of the local packing fraction under shear in a split-bottom ring shear cell using Magnetic Resonance Imaging. They observed that the relative change in the local density in the flowing zone is rather strong. After long times, a large zone, with almost constant, low packing fraction forms, which coincides with the shear band. The local packing fraction remains constant, and independent of local strain rate, suggests that the density of the flowing granular material depends on total strain, similar to what was observed by Kabla [81].

\subsubsection{Segregation}

In this section, segregation studies concerning split-bottom ring shear cell are briefly reviewed. For more details, interested readers are suggested to read [139]. For dense sheared granular mixtures, there are three possible driving mechanisms to drive segregation: gravity, porosity, and velocity gradients. Hill et al. [67] studied segregation of mixture of particles in a split-bottom cell. They find that gravity alone does not drive segregation associated with particle size without a sufficiently large porosity or porosity gradient. A velocity gradient, however, appears to be capable of driving segregation associated with both particle size and material density. In a later study [51], they found that the direction of shear-driven segregation depends on the nature of the flow itself, collisional or frictional. Further studies by Harrington et al. [60] found suppression and emergence of segregation, which was attributed to the presence of a critical shear amplitude that brings about segregation.

\subsubsection{Reflection and exclusion of shear bands}

Unger et al. [207] studied refraction of shear bands in the layered granular materials. They found a new effect for shear bands that are created in layered granular materials. When two materials with different frictional properties are layered on top of each other, shear bands are refracted at the interface [207]. The phenomenon is in complete analogy with the refraction of light. The angle of refraction follows Snell's law from geometric optics. Tamás et al. [19] found that under natural pressure conditions i.e., in the presence of gravity, the shear band can also be deflected by the interface, so that the deformation of the high friction material is avoided. Tamás et al. [20] found that in a layered system with different effective frictions, the presence of material interface leads to a special type of "total internal reflection " of the shear band. However, unlike in optics the zone reflection occurs always at the critical angle of refraction. In case of shear bands this angle is defined by the ratio of the effective frictions of the two material layers. This special reflection also involves a part of the shear band trapped at the interface of the layers. 


\subsection{Fast Flows}

Description of fast dry granular flows, for example steady granular flow down an incline, has made much progress recently. A comprehensive review, see Ref. [55]; a brief review of the main results is presented here. Simple, steady state fluid like properties explains the bulk behavior. On a microscopic level, collisions are mixed between binary collision (as in granular gas) and enduring frictional contacts.

2.2.0.6.1 The inertial number - For the case of infinitely rigid particles (such as glass beads), a simple dimensionless parameter called the inertial number can be constructed using variables which play a role in the flow. The local pressure $p$, the local strain rate $\dot{\gamma}$, the mean particle diameter $d$ and the local density $\rho$ can be combined to give:

$$
I=\frac{\dot{\gamma} d}{\sqrt{p / \rho}}
$$

This number signifies the local 'fastness' of the flow. An elegant interpretation is presented in [118], where it is described as a ratio of two time scales in the granular flows. $1 / \dot{\gamma}$ is the timescale of (shear) strain induced rearrangements of particle in this flow, and $d / \sqrt{\frac{p}{\rho}}$ is the time a particle takes to move over a distance of order $d$, subjected to the force $p d^{2}$. The inertial number is also equivalent to the square root of the Savage number or Coulomb number [165]. It is important to mention that this dimensionless number assumes that particles are hard, otherwise the particle elasticity becomes relevant $[28,138]$.

2.2.0.6.2 Friction law For rigid grains, the shear stress is proportional to the pressure, with effective friction coefficient being a function of $I . \mu(I)$ is an empirical function, and involves the material parameters, given as:

$$
\mu(I)=\mu_{s}+\frac{\mu_{2}-\mu_{1}}{I_{0} / I+1},
$$

where $\mu_{s}$ is the friction coefficient in the limit of very small strain rate, $\mu_{2}$ is the saturation reached for high $I$, and $I_{0}$ is the typical inertial number (reference scale). The saturation of friction coefficient for infinitely large $I$ is supported by the experiments of steady granular front down an inclined plane [147]. This friction law successfully captures many aspects of rapid granular flows $[55,80,118]$.

2.2.0.6.3 Dilatancy law The local volume fraction in a flowing zone is found to decrease with increasing $I$ as

$$
\phi(\mathrm{I})=\phi_{\max }+\left(\phi_{\min }-\phi_{\max }\right) \mathrm{I}
$$


where typical values of $\phi_{\max }$ are close to RCP and $\phi_{\min }$ around 0.7 in two dimensions [35], and 0.55 in three dimensions [55].

Here, we recall few geometries, which were briefly discussed for slow flows in the context of fast flows.

Flow of grains on rough inclined plane has been investigated both experimentally and numerically [118]. Many of the observations can be captured by the local rheology. Using force balance across a flowing layer very well predicts Bagnold velocity profile [55]. In case of plane shear, the stress distribution is homogeneous in the flowing layer, and a linear velocity profile can be predicted. However for Couette flow, the stress distribution inside the lowing zone is similar to that of inclined plane. But the velocity profiles are found to be linear, instead of Bagnold type. Jop performed simulations of flows in split-bottom ring shear cell using the inertial number theory [79]. The center of the shear band in the bulk, and smooth transition to precession, and the dome flow were captured. The width of the shear band was found to scale with the rate of rotation, and for slow flows the shear band width was found to be zero.

The inability of local rheology to predict the width of the shear band for slow flows, and the violation of velocity profile prediction for Couette flow encourages for a non-local description $[119,148,149]$. Recently non-local theory with a fluidity parameter has successfully predicted various flow flow profiles [82]. A size-dependent non-local model introduced recently can predict finite width of shear band [64].

\subsection{Methodology}

\subsubsection{Discrete Element Method (DEM)}

The discrete element method, which allows to simulate large numbers of interacting particles, is the numerical method used in this thesis. We briefly summarize the principle of the method in this section.

A possibility to obtain information about the behavior of granular media is to perform careful experiments. An alternative are simulations with molecular dynamics (MD) or the discrete element method (DEM) [14, 34, 66, 92, 194, 211, 215]. Note that both methods are identical in spirit, however, different names are used by different group of researchers.

The elementary units of bulk granular material are mesoscopic particles which deform under external applied stress/force. Since the realistic modeling of the deformations of the particles is much too complicated, we relate the interaction force to the overlap $\delta$ of two particles. Note that the evaluation of the inter-particle forces based on the overlap may not be sufficient to account for the inhomogeneous stress distribution inside the particles. Consequently, the 
results of DEM simulations are of the same quality as the simple assumptions about the force-overlap relation [1, 34, 100, 102]. For details about DEM simulations readers are referred to [102]. A brief review of various contact models for normal force is presented in Chapter 2, hence is not presented here. Readers interested in contact models for tangential forces should read [102].

\subsubsection{Micro-macro transition}

For scientific research and industrial applications, the major challenge is to obtain continuum constitutive relations from experiments and numerical tests. In other words, the main goal is to find a connection between the microscopic properties and the macroscopic bulk behavior. Bridging the gap between the two involves the so-called micro-macro transition $[11,102$, $104,215]$.

2.3.2.0.4 global-local averaging Extensive "microscopic" simulations of many homogeneous small samples, i.e., so-called representative volume elements (RVE), have to be used to derive the macroscopic constitutive relations needed to describe the material within the framework of a continuum theory [215]. However, it is important to realize that the granular flows are heterogeneous in nature, hence the assumption of homogeneous samples inside a RVE might be misleading. An alternative is to do the local averaging at the level of few grain sizes or even smaller. The approach used in this study is to simulate an inhomogeneous geometry. In such a geometry, granular packings with contrasting properties and behavior co-exist, both high density static areas and dilated dynamic, flowing zones are found in the same system. Using adequate local averaging over equivalent volumes — inside which all particles are assumed to behave similarly, local constitutive relations within a certain parameter range can be obtained using a single numerical experiment. This method has been systematically applied in two-dimensional Couette ring shear cells [91, 92], and three dimensional split-bottom ring shear cells $[103,105]$. Especially in the three dimensional split-bottom ring shear cell, we take the advantage of gravity in the system and critical state yield stress at various various pressure levels can be obtained from a single simulation.

\subsubsection{Averaging and micro-macro procedure}

Translational invariance is assumed in the tangential $\phi$-direction, the averaging is performed over toroidal volumes, over many snapshots in time. leading to field $Q(r, z)$ as function of the radial and vertical positions. The averaging procedure has been explained in detail for 2D systems in [91, 92], and three dimensional systems in [103-105], and will not discussed here. The simulation runs for more than $50 \mathrm{~s}$. For the spatial and time averaging, only large times are taken into account, disregarding the transient behavior at the onset of shear. 
2.3.2.1.1 Stress Tensor From the simulations, one can calculate the stress tensor as

$$
\sigma_{i j}=\frac{1}{V}\left[\sum_{p \in V} m^{p}\left(v_{i}^{p}\right)\left(v_{j}{ }^{p}\right)-\sum_{c \in V} r_{i}^{c} f_{j}^{c}\right]
$$

with $p$ particles, mass $m^{p}$, velocity $v^{p}$, force $f^{c}$ and branch vector $r^{c}$. The velocity $v^{p}$ is relative to the mean streaming velocity inside the averaging volume $V$. The first term is the sum of kinetic energy fluctuations, and the second involves the dyadic product of contactforce with the contact-branch vector.

2.3.2.1.2 Fabric Tensor The quantity which describes the local network of contacts in a granular material is the fabric tensor [130,131], defined as

$$
F_{i j}=\frac{1}{V} \sum_{p \in V} V^{p} \sum_{c \in p} n_{i}^{c} n_{j}^{c}
$$

where $V^{p}$ is the particle volume which lies inside the averaging volume $V, n^{c}$ is the normal unit branch-vector pointing from center of particle $p$ to contact $c$.

For both stress and fabric tensors, we can calculate the eigenvalues and define the volumetric part $T_{\mathrm{V}}=\left(T_{1}+T_{2}+T_{3}\right) / 3$ (pressure $p$ and $F_{\mathrm{V}}$ for stress and fabric respectively) and deviatoric component as $T_{\mathrm{dev}}=\sqrt{\left(\left(T_{1}-T_{2}\right)^{2}+\left(T_{2}-T_{3}\right)^{2}+\left(T_{3}-T_{1}\right)^{2}\right) / 6}\left(\sigma_{\mathrm{dev}}\right.$ and $F_{\mathrm{dev}}$ for stress and fabric respectively).

The pressure is the isotropic stress, while $\sigma_{\mathrm{dev}}$ quantifies the normal stress difference. The volumetric fabric $F_{\mathrm{v}}$ represents the contact number density, while the deviatoric fabric $F_{\mathrm{dev}}$ quantifies anisotropy of the contact network.

In rest of the thesis, local averaging is applied to the steady state data from simulations with different particle and system properties to study their effect on the macroscopic bulk behavior. 


\title{
CHAPTER 3
}

\section{contact model for sticking of adhesive mesoscopic particles*}

\begin{abstract}
The interaction between realistic visco-elasto-plastic and adhesive meso-particles is the subject of this study. The goal is to define a simple, flexible and useful interaction model that allows to describe the multi-contact bulk behavior of assemblies of nonhomogeneous/non-spherical particles, e.g. with internal structures of the scale of their contact deformation. We attempt to categorize previous approaches and propose a simplified mesoscale normal contact model that contains the essential ingredients to describe an ensemble of particles, while it is not aimed to include all details of every single contact, i.e. the mechanics of constituent elementary, primary particles is not explicitly taken into account.
\end{abstract}

The model combines short-ranged, non-contact adhesive interactions with an elaborate, piece-wise linear visco-elasto-plastic adhesive contact law. Using energy conservation arguments, the binary collisions is studied and an analytical expression for the coefficient of restitution in terms of impact velocity is derived, for the special case of very small non-contact force. The assemblies (particles or meso-particles) stick to each other at very low impact velocity, while they rebound less dissipatively with increasing velocity, in agreement with previous findings for elasto-plastic spherical particles. For larger impact velocities we observe a second sticking regime. The first

*. Based on A. Singh, V. Magnanimo, and S. Luding. Contact model for sticking of adhesive mesoscopic particles. Powder Technology, Under Review, 2013 
sticking is attributed to dominating non-contact adhesive forces, while the high velocity sticking is due to a balance between the non-linearly increasing history dependent ahdesion and plastic dissipation. The model allows for a stiff, elastic core material, which produces a new rebound regime at even higher velocities.

The relevance of the model for various types of bulk materials is critically discussed with respect to features as: non-linear pressure dependent bulk stiffness, limit elasticity vs plasticity or non-perfect detachment under slow tension. 


\section{Nomenclature}

$m_{i} \quad: \quad$ mass of $i^{t h}$ particle.

$a_{i} \quad: \quad$ Radius of $i^{\text {th }}$ particle.

$m_{r} \quad$ : Reduced mass of two particles.

$\delta \quad:$ Contact overlap between particles.

$k \quad$ : Spring stiffness.

$v_{i} \quad$ : Relative velocity before collision.

$v_{f}:$ Relative velocity after collision.

$v_{i}{ }^{\infty} \quad$ : Relative velocity before collision at infinite separation.

$v_{f}{ }^{\infty}$ : Relative velocity after collision at infinite separation.

$v^{n} \quad$ : Normal component of relative velocity.

$e \quad:$ Coefficient of restitution.

$\epsilon_{i} \quad:$ Pull-in coefficient of restitution.

$e_{n} \quad: \quad$ Normal coefficient of restitution.

$\epsilon_{o} \quad:$ Pull-off coefficient of restitution.

$k_{1} \quad$ : Slope of loading plastic branch.

$k_{2} \quad: \quad$ Slope of unloading and re-loading elastic branch.

$k_{p} \quad: \quad$ Slope of unloading and re-loading limit elastic branch.

$k_{c} \quad:$ Slope of irreversible, tensile adhesive branch.

$v_{p} \quad$ : Relative velocity before collision for which the limit case of overlap is reached.

$\phi_{f} \quad$ : Dimensionless plasticity depth.

$\delta_{\max }:$ Maximum overlap between particles for a collision.

$\delta_{\max }^{p} \quad: \quad$ Maximum overlap between particles for the limit case.

$\delta_{0} \quad: \quad$ Force free overlap $\cong$ plastic contact deformation.

$\delta_{\min }:$ Overlap between particles at the maximum negative attractive force.

$\delta_{\mathrm{c}} \quad: \quad$ Kinetic Energy free overlap between particles.

$W_{\text {diss }}$ : Amount of energy dissipated during collision.

$\eta \quad$ : Dimensionless plasticity of the contact.

$\beta \quad$ : Adhesivity: dimensionless adhesive strength of the contact.

$\chi \quad$ : Scaled initial velocity relative to $v_{p}$.

$f_{a}:$ Non-contact adhesive force at zero overlap.

$\delta_{\mathrm{a}} \quad$ : Non-contact separation between particles at which attractive force becomes active.

$k_{c}{ }^{a} \quad$ : Strength of non-contact adhesive force.

\subsection{Introduction}

Flows of granular materials are ubiquitous in industry and nature. For this reason, the past decade has witnessed a strong interest in better understanding of their behavior. Especially, the impact of fine particles with particles/surfaces is a fundamental problem. The interaction 
force between two particles is a combination of elasto-plastic deformation, viscous dissipation, and adhesion - due to both contact and long-range non-contact forces. Pair interactions that can be used in bulk simulations with many contacts per particle are the focus, and we use the singular special case of pair interaction to understand them.

Different regimes are observed for two colliding particles: For example a particle can either stick to another particle/surface or it rebounds, depending upon the relative strength of adhesion and impact velocity, size and material parameters. This problem needs to be studied in detail, as it forms the base for understanding more complex, many-particle flows in realistic systems, related to e.g. astrophysics (dust agglomeration, Saturn's rings, planet formation) or industrial processes (handling of fine powders, granulation, filling and discharging of silos). Particularly interesting is the interaction mechanism for adhesive materials such as asphalt, ice particles or clusters/agglomerates of fine powders (often made of even smaller primary particles). Some materials can be physically visualized as having a plastic outer shell with a rather stiff, elastic inner core. Moreover, the analysis can be applied to particle-surface collisions in kinetic spraying, in which the solid micro-sized powder is accelerated towards a substrate. In cold spray, bonding occurs when impact velocities of particles exceed a critical value, that depends on various material parameters $[168,189,232]$ but for even larger velocities particles rebound [228, 229]. Due to the inhomogeneity of most realistic materials, their non-sphericity, and their surface irregularity, the goal is not to include all the possible details - but rather to catch the essential phenomena and ingredients, finding a compromise between simplicity and realistic contact mechanics.

\subsubsection{Contact Models Review}

Computer simulations have turned out to be a powerful tool to investigate the physics of particulate systems, especially valuable as there is no generally accepted theory of granular flows so far, and experimental difficulties are considerable. A very popular simulation scheme is an adaptation of the classical Molecular Dynamics technique called Discrete Element Method (DEM) (for details see Refs. [14, 34, 66, 92, 101, 102, 215]). It consists of integrating Newton's equations of motion for a system of "soft", deformable grains, starting from a given initial configuration. DEM can be successfully applied to adhesive particles, if a proper force-overlap model (contact model) is given.

Brilliantov et al. [23] investigated the collision of adhesive viscoelastic spheres and presented a general analytical expression for their collision dynamics, but we rather turn to plastic contact deformations in the following. The JKR model [78] is a widely accepted adhesion model for elastic spheres and gives an expression for the normal force. Later, Derjaguin et al. [40] considered that the attractive forces act only just outside the contact zone, where surface separation is small. One interesting model for dry adhesive particles was proposed by Molerus [120,121], which explained consolidation and non-rapid flow of adhesive 
particles in terms of adhesion forces at particle contacts. Thornton and Yin [198] compared the results of elastic spheres with and without adhesion and Thornton, later on in Ref. [197], extended this work to adhesive elasto-plastic spheres. Molerus's model was further developed by Tomas, who introduced a contact model [201-203] that couples elasto-plastic contact behavior with non-linear adhesion and hysteresis, which involves dissipation and a history (compression) dependent adhesive force. The contact model subsequently proposed by Luding [102, 109, 110] works in the same spirit as the one of Tomas [201], only reducing complexity by using piece-wise linear branches in an otherwise still highly non-linear contact model. In [102], the short ranged non-contact force beyond contact was not specified as in the present study. Complex details like a possible non-linear Hertzian law for small deformation, and non-linear load-unload hysteresis are over-simplified in the model as compared to Tomas [201]. This is partly due to the lack of experimental reference data or theories, but also to keep the model as simple as possible. The model contains the basic mechanisms, elasticity, plasticity and adhesion as relevant for fine, dry powders and shell-core materials. A possible connection between the microscopic contact model and the macroscopic, continuum description for adhesive particles was recently proposed by Luding and AlonsoMarroquin [107]. Walton et al. [218, 223] proposed a contact model which works in the same spirit as that of Luding [102] and Tomas [201], but separates the rate of pull-off force from the slope of tensile attractive force. Jiang et al. [75] experimentally investigated the force-displacement behavior of idealized bonded granules, which was later implemented in DEM in [76] to study the mechanical behavior of loose cemented granular material. Kempton et al. [84] proposed a meso-scale contact model combining linear hysteretic, simplified JKR and linear bonding force models, to simulate agglomerates of sub-particles. Recently a contact model is proposed by Thakur et al. [193] , which works in the same spirit as Luding's model, but treats loading and un/re-loading behaviors non-linearly.

When two particles collide, the behavior is intermediate between the extremes of perfectly elastic and fully inelastic, possibly fragmenting collisions. The elasticity of the collision can be best described by the coefficient of restitution, which is the ratio of magnitude of post-collision and pre-collision normal relative velocities of the particles. It quantifies the amount of energy not dissipated during the collision. For the case of plastic and viscoelastic collisions, it was suggested that dissipation should be dependent on impact velocity $[77,89$, 221], as can be realized by viscoelastic forces [25, 89, 99, 100] and follows from plastic deformations too [235]. An early experimental study on micrometer adhesive polystyrene latex spheres was done by Dahneke [36]. He observed sticking of adhesive particles for low velocities, and an increasing coefficient of restitution for velocity higher than a critical threshold. Wall et al. [217], confirmed these findings for highly mono-disperse ammonium particles. Thornton et al. [197] and Brilliantov et al. [23] presented adhesive visco-elastoplastic contact models in agreement with these experiments. Work by Sorace et al. [186] further confirms the sticking at low velocities for particle sizes of the order of a few mm. Jasevičius et al. [73, 74] have recently presented the rebound behavior of ultrafine silica 
particles using the contact model from Refs. [201-204].

\subsubsection{Model classification}

Since our focus is on dry particles, we do not review the diverse work involving liquid [65] or strong solid bridges [21] here. Even though oblique collisions between two particles are of practical relevance and have been studied in detail by Thornton et al. [196], here we focus on central normal collisions without loss of generality. Finally, we also disregard many details of non-contact forces, as e.g. due to van der Waals forces, for the sake of brevity, but will propose a simple mesoscale non-contact force model in section 3.2.3.

Based on our review of adhesive, elasto-visco-plastic contact models, we propose here a (partial) classification, by dividing them into three groups: (1) Academic, (2) Mesoscopic, and (3) Realistic, detailed contact models. Here we review adhesive elastic, and elastoplastic contact models only. A detailed review concerning the effects of various forces on adhesion of fine particles is nicely reviewed in [219].

1. Academic contact models allow for easy analytical solution, as for example the linear spring-dashpot model [100], or piece-wise linear models with constant unloading stiffness (see e.g. Walton and Braun [222] (constant coefficient of restitution mode)), which feature a constant coefficient of restitution, independent of impact velocity. Also the Hertzian visco-elastic models, belong to this class, even though they provide a velocity dependent coefficient of restitution, for a summary see [100]. However, no academic model describes particle deformation that would be practically relevant, as their range of strict validity is extremely limited.

2. Mesoscopic contact models are a compromise, (i) still rather easy to implement, (ii) aimed for fast ensemble/bulk-simulations with many particles and various materials, but (iii) not matching all the minute contact details of every single contact, they are often piece-wise linear, e.g., with a variable unloading stiffness or with an extended adhesive force (Walton and Braun [222] (variable coefficient of restitution mode), Luding [102], Walton [223], Thakur et al. [193]).

3. Realistic, full-detail contact models have (i) the most realistic, but often rather complicated formulation, (ii) can reproduce with similar precision the pair interaction and the bulk behavior, but (iii) are valid only for the limited class of materials they are particularly designed for, since they do include all the minute details of these interactions. As a few examples, there are:

(a) visco-elastic models: Walton [220], Brilliantov [23, 24], Haiat [59];

(b) adhesive elastic models: JKR [78], DMT [40], Thornton and Yin [198];

(c) adhesive elasto-plastic models: Molerus[120], Thornton and Ning [197], Tomas [201-204]. 
Since our main goal is to define and apply contact models to simulate the bulk behavior of assemblies of many fine particles (for which no valid realistic model is available), we focus on the second class, mesoscopic models.

\subsubsection{Focus and Overview of this study}

In particular, we study the dependence of the coefficient of restitution for two meso-particles on impact velocity and contact/material parameters, for a wide range of impact velocities, using a generalized version of the contact model by Luding [102], extended by a non-contact force term. We observe sticking of particles at low velocity, which is consistent with previous theoretical and experimental works [186, 197, 217]. Pasha et al. [142] recently also reproduced the low velocity sticking using an extension of a similar, simpler model [109]. Above a certain small velocity, dissipation is not strong enough to dissipate all relative kinetic energy and the coefficient of restitution increases. We want to understand the full regime of relative velocities, and thus focus also on the less explored intermediate and high velocity regimes, as easily accessible in numerical simulations. In the intermediate regime, we observe a decrease of the coefficient of restitution, as observed previously for idealized particles, see e.g. Refs. [23, 197], but with different functional behavior as predicted by some realistic models, a property that can be tuned by simple modifications to the mesoscopic model. With further increase in impact velocity, we find a second interesting sticking regime due to the increasing adhesive dissipation with respect to plastic dissipation. Finally, since the physical systems under consideration also are viscous in nature, we conclude with some simulations with added viscous damping.

An exemplary application of our model that leads to the unexpected high velocity sticking and rebound regimes is, among others, the study of coating processes in cold sprays, where researchers are interested in the effect of the impact velocity on the deposition efficiency of the powder on a substrate. In this process, bonding/coating happens when the impact velocity of the particles exceeds a critical velocity, with values in the order $10^{2} \mathrm{~m} / \mathrm{s}$. Interestingly, when the velocity is further increased the particles do not bond to the substrate anymore, with a decrease of the deposition efficiency (inverse of coefficient of restitution) [228]. Schmidt et al. [168] have used numerical simulations to explore the effect of various material properties on the critical velocity, while Zhou et al. [229] studied the effect of impact velocity and material properties on the coating process. The data show that properties of both particle and substrate influence the rebound, the details of which go beyond the scope of this study. Using our model we can explore the dependence of the deposition efficiency on the impact velocity, helping the interaction between different communities.

In section 3.2, we introduce the DEM simulation method and the basic normal contact models, which are further elaborated on in the following section 3.3, where the coefficient of restitution is computed. Dimensionless contact parameters are proposed in section 3.4.1, the 
dependence on contact adhesion is described in section 3.4.2, the effect of viscosity in section 3.A.1, and some asymptotic solutions are given in section 3.A.2. The study is concluded in section 3.5 .

\subsection{Discrete Element Method}

The elementary units of granular materials are mesoscopic grains, which deform under stress. Since the realistic and detailed modeling of real particles in contact is too complicated, it is necessary to relate the interaction force to the overlap $\delta$ between two particles. Note that the evaluation of the inter-particle forces based on the overlap may not be sufficient to account for the inhomogeneous stress distribution inside the particles and possible multi-contact effects [77]. This price has to be paid in order to simulate larger samples of particles with a minimal complexity and still taking various physical contact properties like non-linear contact elasticity, plastic deformation or load-dependent adhesion into account.

\subsubsection{Equations of Motion}

If all forces acting on a spherical particle $p$, either from other particles, from boundaries or externally, are known - let their vector sum be $f_{p}$, then the problem is reduced to the integration of Newton's equations of motion for the translational degrees of freedom (the rotational degrees are not considered here since we focus on normal forces) for each particle:

$$
m_{p} \frac{\mathrm{d}^{2}}{\mathrm{~d} t^{2}} r_{p}=f_{p}+m_{p} g
$$

where, $m_{p}$ is the mass of particle $p, r_{p}$ its position, $f_{p}=\sum_{c} f_{p}^{c}$ is the total force due to all contacts $c$, and $g$ is the acceleration due to volume forces like gravity.

With tools from numerical integration, solving the equations of motion, as nicely described in textbooks as $[1,146]$, is a straightforward exercise. The typically short-ranged interactions in granular media allow for further optimization by using linked-cell (LC) or alternative methods in order to make the neighborhood search more efficient. However, this is not of concern in this study, since only normal pair collisions are considered.

\subsubsection{Normal Contact Force Laws}

Two spherical particles $i$ and $j$, with radii $a_{i}$ and $a_{j}, r_{i}$ and $r_{j}$ being the position vectors respectively, interact if they are in contact so that their overlap,

$$
\delta=\left(a_{i}+a_{j}\right)-\left(r_{i}-r_{j}\right) \cdot n
$$

is positive, $\delta>0$, with the unit vector $n=n_{i j}=\left(r_{i}-r_{j}\right) /\left|r_{i}-r_{j}\right|$ pointing from $j$ to $i$. The force on particle $i$, from particle $j$, at contact $c$, can be decomposed into a normal 


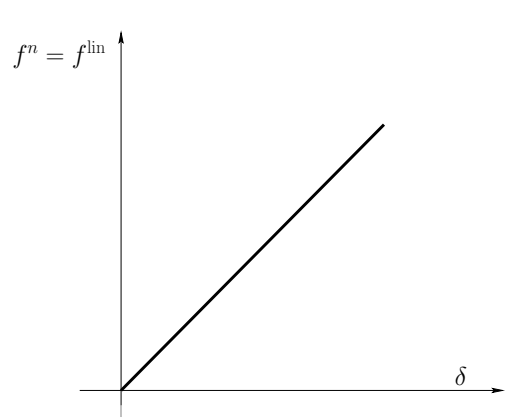

(a)

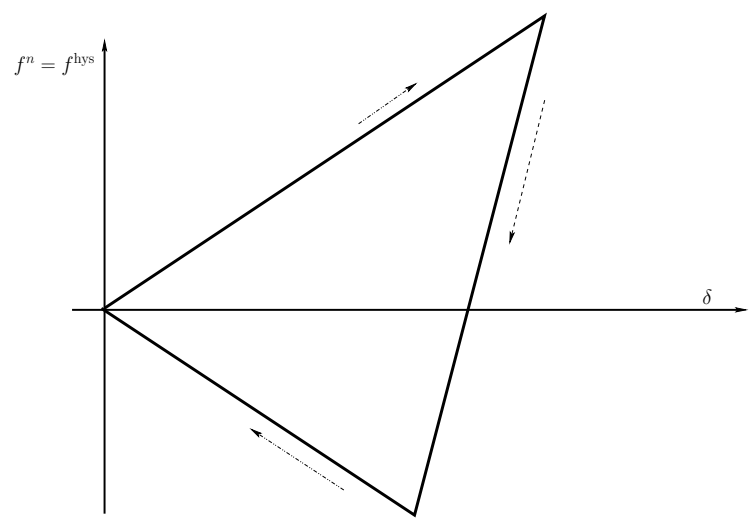

(b)

Figure 3.1: Schematic plots of (a) the linear normal contact model for a perfectly elastic collision, and (b) the force-overlap relation for an elasto-plastic adhesive collision

and a tangential part as $f^{c}:=f_{i}^{c}=f^{n} n+f^{t} t$, where $n \cdot t=0, n$ and $t$ being normal and tangential parts respectively. In this chapter, we focus on frictionless particles, that is only normal forces will be considered, for tangential forces and torques, see e.g. Ref. [102] and references therein.

In the following, we discuss various normal contact force models, as shown schematically in Fig. 3.1. We start with the linear contact model (Fig. 3.1(a)) for non-adhesive particles, before we introduce a more complex contact model that is able to describe the realistic interaction between adhesive, inhomogeneous, slightly non-spherical particles (Fig. 3.1(b)).

\subsubsection{Linear Normal Contact Model}

Modelling a force that leads to an inelastic collision requires at least two ingredients: repulsion and some sort of dissipation. The simplest normal force law with the desired properties is the damped harmonic oscillator

$$
f^{n}=k \delta+\gamma_{0} v^{n}
$$

with spring stiffness $k$, viscous damping $\gamma_{0}$, and normal relative velocity $v^{n}=-\vec{v}_{i j} \cdot \vec{n}=$ $-\left(\vec{v}_{i}-\vec{v}_{j}\right) \cdot \vec{n}=\dot{\delta}$. This model (also called linear spring dashpot (LSD) model) has the advantage that its analytical solution (with initial conditions $\delta(0)=0$ and $\dot{\delta}(0)=v_{0}^{n}$ ) allows the calculations of important quantities very easily [100]. For the non-viscous case, the linear normal contact model is given schematically in Fig. 3.1a.

The typical response time (contact duration) and the eigenfrequency of the contact are related 
as

$$
t_{c}=\frac{\pi}{\omega} \quad \text { and } \quad \omega=\sqrt{\left(k / m_{r}\right)-\eta_{0}^{2}}
$$

with the rescaled damping coefficient $\eta_{0}=\gamma_{0} /\left(2 m_{r}\right)$, and the reduced mass $m_{r}=m_{i} m_{j} /\left(m_{i}+\right.$ $m_{j}$ ). From the solution of the equation of a half-period of the oscillation, one also obtains the coefficient of restitution

$$
e_{n}^{\mathrm{LSD}}=v_{f} / v_{i}=\exp \left(-\pi \eta_{0} / \omega\right)=\exp \left(-\eta_{0} t_{c}\right)
$$

which quantifies the ratio of normal relative velocities after $\left(v_{f}\right)$ and before $\left(v_{i}\right)$ the collision. Note that in this model $e_{n}$ is independent of $v_{i}$. For a more detailed review on this and other, more realistic, non-linear contact models, see [100, 102] and references therein.

The contact duration in Eq. (3.4) is also of practical and technical importance, since the integration of the equations of motion is stable only if the integration time-step $\Delta t$ is much smaller than $t_{c}$. Note that $t_{c}$ depends on the magnitude of dissipation: In the extreme case of an over-damped spring (high dissipation), $t_{c}$ can become very large (which renders the contact behavior artificial [99]). Therefore, the use of neither too weak nor too strong viscous dissipation is recommended.

\subsubsection{Adhesive Elasto-Plastic Contacts}

Here we apply a variation to previously proposed piece-wise linear hysteretic model [100 $102,202,222]$ as an alternative to non-linear spring-dashpot models or more complex hysteretic models [197, 201-203, 216]. It reflects permanent plastic deformation, which might take place at the contact, and stronger attractive (adhesive) forces, both depending nonlinearly on the maximal compression force.

In Fig. 3.2, the normal force at contact is plotted against the overlap $\delta$ between two particles. The force law can be written as

$$
f^{\text {hys }}= \begin{cases}k_{1} \delta & \text { if } k_{2}\left(\delta-\delta_{0}\right) \geq k_{1} \delta \\ k_{2}\left(\delta-\delta_{0}\right) & \text { if } k_{1} \delta>k_{2}\left(\delta-\delta_{0}\right)>-k_{c} \delta \\ -k_{c} \delta & \text { if }-k_{c} \delta \geq k_{2}\left(\delta-\delta_{0}\right)\end{cases}
$$

with $k_{1} \leq k_{2} \leq k_{p}$, respectively the initial loading stiffness, the un-/re-loading stiffness and the elastic limit stiffness. The latter defines the limit force branch $k_{p}\left(\delta-\delta_{0}^{p}\right)$, as will be motivated below in more detail, and $k_{2}$ is interpolating between $k_{1}$ and $k_{p}$, see Eq. (5.9). For $k_{c}=0$, the above contact model reduces to that proposed by Walton and Braun [222], which leads to a variable coefficient of restitution

$$
e_{n}^{\mathrm{WB}}=\sqrt{k_{1} / k_{2}},
$$

as proposed by Walton and Braun [222]. 


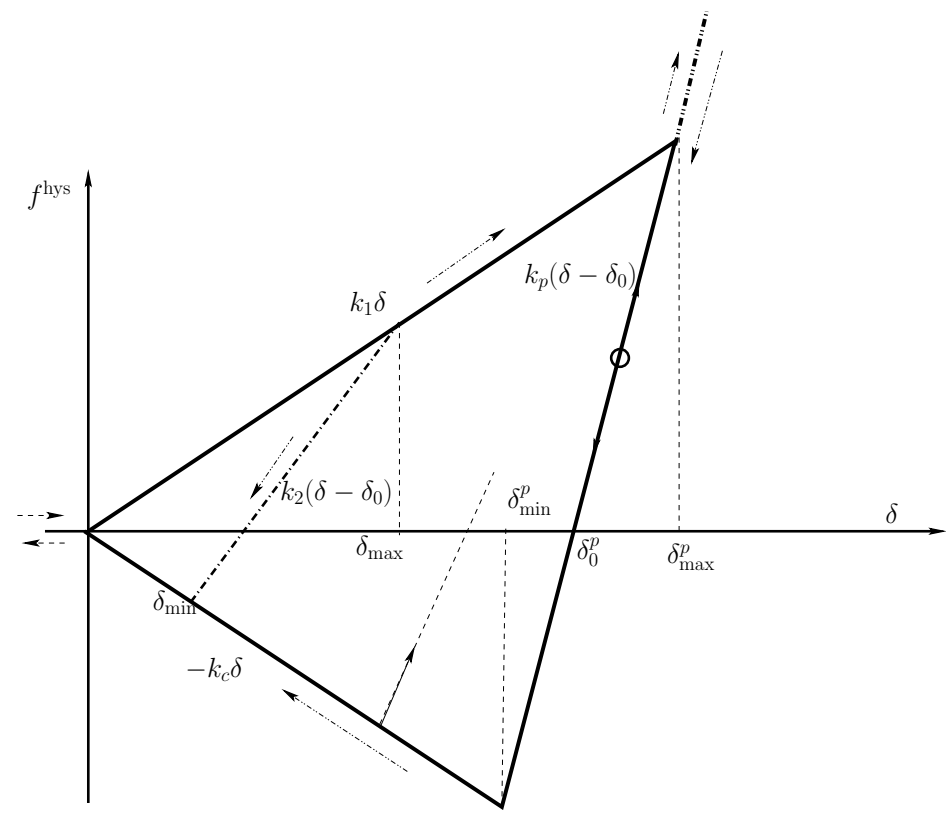

Figure 3.2: Schematic graph of the piece-wise linear, hysteretic, and adhesive forcedisplacement model in normal direction.

During the initial loading the force increases linearly with overlap $\delta$ along $k_{1}$, until the maximum overlap $\delta_{\max }=v_{i} \sqrt{m_{r} / k_{1}}$ (for binary collisions) is reached, which is a history parameter for each contact. During unloading the force decreases along $k_{2}$, see Eq. (5.9), from its maximum value $k_{1} \delta_{\max }$ at $\delta_{\max }$ down to zero at overlap

$$
\delta_{0}=\left(1-k_{1} / k_{2}\right) \delta_{\max },
$$

where $\delta_{0}$ resembles the permanent plastic contact deformation. Reloading at any instant leads to an increase of the force along the (elastic) branch with slope $k_{2}$, until the maximum overlap $\delta_{\max }$ (which was stored in memory) is reached; for still increasing overlap $\delta$, the force again increases with slope $k_{1}$ and the history parameter $\delta_{\max }$ has to be updated.

Unloading below $\delta_{0}$ leads to a negative, attractive (adhesive) force, which follows the line with slope $k_{2}$, until the extreme adhesive force $-k_{c} \delta_{\min }$ is reached. The corresponding overlap is

$$
\delta_{\min }=\frac{\left(k_{2}-k_{1}\right)}{\left(k_{2}+k_{c}\right)} \delta_{\max } .
$$

Further unloading follows the irreversible tensile branch, with slope $-k_{c}$, and the attractive force $f^{\text {hys }}=-k_{c} \delta$.

The lines with slope $k_{1}$ and $-k_{c}$ define the range of possible force values and departure from 
these lines takes place in the case of unloading and re-loading, respectively. Between these two extremes, unloading and re-loading follow the line with slope $k_{2}$. A non-linear un-/reloading behavior would be more realistic, however, due to a lack of detailed experimental informations, the piece-wise linear model is used as a compromise; also it is easier to implement. The elastic $k_{2}$ branch becomes non-linear and ellipsoidal, when a moderate normal viscous damping force is active at the contact, as in the LSD model.

In order to account for realistic load-dependent contact behavior, the $k_{2}$ value is chosen dependent on the maximum overlap $\delta_{\max }$, i.e. particles are more stiff for larger previous deformation and the dissipation is dependent on deformation. The dependence of $k_{2}$ on overlap $\delta_{\max }$ is chosen empirically as linear interpolation (in the same spirit as Walton and Braun [222]):

$$
k_{2}\left(\delta_{\max }\right)= \begin{cases}k_{p} & \text { if } \delta_{\max } / \delta_{\max }^{p} \geq 1 \\ k_{1}+\left(k_{p}-k_{1}\right) \delta_{\max } / \delta_{\max }^{p} & \text { if } \delta_{\max } / \delta_{\max }^{p}<1\end{cases}
$$

where $k_{p}$ is the (maximal) elastic stiffness, and

$$
\delta_{\max }^{p}=\frac{k_{p}}{k_{p}-k_{1}} \phi_{f} \frac{2 a_{1} a_{2}}{a_{1}+a_{2}},
$$

is the plastic flow limit overlap, with $\phi_{f}$ the dimensionless plasticity depth, $a_{1}$ and $a_{2}$ being the radii of the two particles. This can be further simplified to

$$
\delta_{0}^{p}=\phi_{f} a_{12}
$$

where $\delta_{0}^{p}$ represents the plastic contact deformation at the limit overlap, and $a_{12}=\frac{2 a_{1} a_{2}}{a_{1}+a_{2}}$ is the reduced radius. In the range $\delta_{\max }<\delta_{\max }^{p}$, the dependence of $k_{2}$ can also be written as follows:

$$
k_{2}=k_{1}+\frac{\left(k_{p}-k_{1}\right)}{k_{1} \delta_{\max }^{p}} f^{\max }
$$

where $f^{\max }=k_{1} \delta_{\max }$, which is same as equation 4 in [222] with $S=\frac{\left(k_{p}-k_{1}\right)}{k_{1} \delta_{\max }^{p}}$.

From energy balance, one can define the "plastic" limit velocity

$$
v_{p}=\sqrt{k_{1} / m_{r}} \delta_{\max }^{p},
$$

below which the contact behavior is elasto-plastic, and above which the perfectly elastic limit-branch is reached. Impact velocities larger than $v_{p}$ can have consequences, as discussed next. 
In summary, the adhesive, elasto-plastic, hysteretic normal contact model is defined by the four parameters $k_{1}, k_{p}, k_{c}$ and $\phi_{f}$ that, respectively, account for the initial plastic loading stiffness, the maximal, plastic limit (elastic) stiffness, the adhesion strength, and the plastic overlap-range of the model; it also involves an empirical choice for the non-linear, loaddependent, intermediate elastic branch stiffness $k_{2}$.

\subsubsection{Motivation of the original contact model}

To study a collision between two ideal, homogeneous spheres, one should refer to realistic, full-detail contact models with a solid experimental and theoretical foundation [78, 197, 202]. These contact models feature a small elastic regime and the particles increasingly deform plastically with increasing, not too large deformation (overlap). During unloading, their contacts end at finite overlap due to flattening. Otherwise, besides many smaller model details there exist various such models. However, one has to consider also the non-contact forces that are often neglected for very large particles, but become dominant and hysteretic as well as long-ranged for rather small spheres [202]. A mesoscopic model that compromises on the details of the contact model, but follows the flat contact detachment philosophy was recently proposed in Ref. [142].

The mesoscopic contact model used here was originally proposed by Luding [102] and follows a different approach in two respects: (i) it introduces a limit to the plastic deformation of the particles/material for various reasons as summarized below in subsection 3.2.2.4, and (ii) the contacts are not idealized as perfectly flat, and thus do not have to lose mechanical contact immediately at un-loading, as will be detailed in the subsection 3.2.2.5.

Note that a limit to the slope $k_{p}$ that resembles a different contact behavior at large deformations has various physical and numerical reasons:

(0) due to the wide probability distribution of forces in bulk granular matter, only few contacts should reach the limit, which should not effect much the collective behavior;

(i) in many particle systems, for large deformations the particles cannot be assumed to be spherical anymore, as they deform plastically or even could break;

(ii) from the macroscopic point of view, too large deformations would lead to volume fractions larger than unity, which for most materials (except highly micro-porous, fractal ones) would be unaccountable;

(iii) at small deformation, contacts are due to surface roughness realized by multiple surface asperities and at large deformation, the single pair point-contact argument breaks down and multiple contacts of a single particle can not be assumed to be independent anymore;

(iv) numerically in a bulk simulation the time step is chosen such that it is well below the minimal contact duration of all the contacts. If $k_{2}$ is not limited this minimum could become very small so that the required time step would have to be reduced below practical values, 
only because of some extreme contacts.

Following two subsections discuss the two major differences of the present piece-wise linear model as compared to other existing models: (i) the elastic limit branch, and (ii) the elastic re-loading or non-contact-loss, as well as their reasons, relevance and possible changes/tuning - in cases needed.

\subsubsection{Shortcomings, physical relevance and possible tuning}

In the context of collisions between perfect homogeneous elasto-plastic spheres, a purely elastic threshold/limit and enduring elastic behavior after a sharply defined contact-loss are indeed questionable, as the plastic deformation of the single particle cannot become reversible/elastic. Nevertheless, there are many materials that support the idea of a more elastic behavior at very high impact velocity (or contact force).

In the following, we will list some of these cases and extensively comment on them.

3.2.2.4.1 Mesoscopic contact model applied to real materials: First we want to recall that the present model is mainly aimed to reproduce the behavior of multi-particle systems of realistic fine and ultra-fine powders, which are typically non-spherical and often mesoscopic in size with internal micro-structure and micro-porosity on the scale of typical contact deformation. For example, think of clusters/agglomerates of primary nano-particles that form fine micron-sized secondary powder particles, or other fluffy materials. The primary particles are possibly better described by other contact models, but in order to simulate a reasonable number of secondary particles one cannot rely on this bottom-up approach and better uses a meso-contact model. During the bulk compression of such a system, the material deforms plastically and its internal porosity reduces. Plastic deformation diminishes if the primary particles are elastic/stiff and the material has become dense, almost non-porous. Beyond this point the system deform more elastically, i.e. the stiffness becomes high and the (irrecoverable) deformations are much smaller than initially.

In their compression experiments of granular beds with micrometer sized granules of microcrystalline cellulose, Persson et al. [143] found that a contact model where a limit on plastic deformation is introduced can very well describe the bulk behavior. Experimentally they observe a strong elasto-plastic bulk-behavior for the assembly at low compression strain/stress. In this phase the height of the bed decreases, irreversibly and almost linearly with the applied load, while the deviation from linearity strongly increases beyond a certain strain/stress, with a dramatic increase of the stiffness of the aggregate. They associate the change in the behavior to the loss of porosity and the subsequent more elastic bulk response to the particles that are now closely in touch with each other. In this new, considerably denser configurations, not much more void reduction is allowed and thus the behavior gets more elastic. While the elastic limit in the contact model does not affect the description of the bulk behavior in the 
first part, the threshold is found to play a key role in order to reproduce the material stiffening (see Fig. 8 in Ref. [143]).

Note that in an assembly of particles, not all the contacts will reach the limit branch and deform elastically simultaneously. That is, even if few contacts are in the elastic limit, the system will always retain some plasticity, hence the assembly will never be fully elastic.

3.2.2.4.2 Application to pair interactions: Interestingly, the contact model in Sec. 3.2.2.2 is suitable to describe the collision between pairs of particles, when special classes of materials are considered, such that the behavior at high velocity and thus large deformation drastically changes.

(i) Core-shell materials. The model is perfectly suited for plastic core-shell materials, such as asphalt or ice particles, having a "soft" plastic outer shell and a rather stiff, elastic inner core. For such materials the stiffness increases with the load due to an increasing contact surface. For higher deformations, contact between the inner cores can take place, which turns out to be almost elastic when compared to the behavior of the external shell. The model was successfully applied to model asphalt, where the elastic inner core is surrounded by a plastic oil or bitumen layer [136]. Alternatively, the plastic shell can be seen as the range of overlaps, where the surface roughness and inhomogeneities lead to a different contact mechanics as for the more homogeneous inner core.

(ii) Cold spray. An other interesting system that can be effectively reproduced by introducing an elastic limit in the contact model is cold spray. Researchers have experimentally and numerically shown that spray-particles rebound from the substrate at low velocity, while they stick at intermediate impact energy [122, 168, 189, 232]. Wu et al. [228] experimentally found that rebound re-appears with a further increase in velocity (Fig. 3 in Ref. [228]). Schmidt et al. [168] relate the decrease of the deposition efficiency (inverse of coefficient of restitution) to a transition from a plastic impact to hydrodynamic penetration (Fig. 16 in Ref. [168]). Recently Moridi et al. [122] numerically studied the sticking and rebound processes, by using the adhesive elasto-plastic contact model of Luding [102], and their prediction of the velocity dependent behavior is in good agreement with experiments.

(iii) Sintering. As an additional example, we want to recall that the present meso-contact model has already been applied to the case of sintering, see Ref. [110]. For large deformations, large stresses, or high temperatures, the material goes to a fluid-like state rather than being solid. Hence, the elasticity of the system (nearly incompressible melt) determines its limit stiffness, while $\phi_{f}$ determines the maximal volume fraction that can be reached.

All the realistic situations described above clearly hint at a modification in the contact phenomenology that can not be described anymore by an elasto-plastic model beyond some threshold in the overlap/force. The limit stiffness $k_{p}$ and the plastic layer depth $\phi_{f}$ in our model allow the transition of the material to a new state. Dissipation on the limit branch 
- which otherwise would be perfectly elastic - can be taken care of, by a viscous damping force (as the simplest option). Due to viscous damping, the unloading and re-loading will follow different paths, so that the collision will never be perfectly elastic, which is in agreement with the description in Jasevičius et al. $[73,74]$ and will be shown below in subsection 3.A.1.

Finally, note that an elastic limit branch is surely not the ultimate solution, but a simple first model attempt - possibly requiring material- and problem-adapted improvements in the future.

3.2.2.4.3 Tuning of the contact model: The change in behavior at large contact deformations is thus a feature of the contact model which allows us to describe many special types of materials. Nevertheless, if desired, without changing the model, the parameters can be tuned in order to reproduce the behavior of materials where the plasticity keeps on increasing with deformation. The limit-branch where plastic deformation ends is defined by the dimensionless parameters plasticity depth, $\phi_{f}$, and (maximal) elastic stiffness, $k_{p}$. Owing to the flexibility of the model, it can be tuned such that the limit overlap is set to a much higher value which is never reached by the contacts. When the new value of $\phi_{f}^{\prime}$ is chosen, a new $k_{p}{ }^{\prime}$ can be calculated to describe the behavior at higher overlap (as detailed in Appendix 3.C). In this way the model with the extended $\phi_{f}^{\prime}$ exhibits elasto-plastic behavior for a higher velocity/compression-force range, while keeping the physics of the system for smaller overlap identical to the one with small $\phi_{f}$.

\subsubsection{Irreversibility of the tensile branch}

Finally we discuss a feature of the contact model in [102], that postulates the irreversibility, i.e. partial elasticity, of the tensile $k_{c}$ branch, as discussed in Sec. 3.2.2.2. While this is unphysical in some situations, e.g. for homogeneous plastic spheres, we once again emphasize that we are interested in non-homogeneous, non-spherical meso-particles, as e.g. clusters/agglomerates of primary particles in contact with internal structures of the order of typical contact deformation.

Only for ideal, perfect, elasto-plastic adhesive spheres that experience a large enough tensile force, the particles detach with a (perfect) flattened surface due to plasticity. In almost all other cases, the shape of the detaching surfaces and the subsequent behavior depend on the relative strength of plastic, attractive, and other contact mechanisms. Moreover, other details like particle rotation can also play a role. Few examples include core-shell materials [136], assemblies of micro-porous fine powders [143] or atomic nanoparticles [191]. We first briefly discuss the case of ideal elasto-plastic adhesive particles and later describe the behavior of many particle systems, which is the main focus of this work.

For ideal homogeneous particles with radius of the order of millimeters [197], a permanently 
flattened surface is created during deformation of the order of micrometers and the plastic dissipation during mechanical contact is dominant with respect to the van der Waals force. When the particles detach during unloading, the force suddenly drops to zero from the tensile branch. During non-contact, further un- and re-loading involves no force; when the contact is re-established it also is assumed to be mostly elastic, following the previous contactunloading path. Thus re-established contacts have little or no plastic deformation until the (previously reached) maximum overlap is reached again - from when on strong plasticity kicks in again.

On the other hand for ultra-fine ideal spherical particles of order of macro-meters [202, 204, 205], the van der Waals force is much stronger and unloading adhesion is due to purely non-contact forces. Therefore, the non-contact forces do not vanish and even extend beyond the mechanical first contact distance. The contact model of Tomas [202, 204] is reversible for non-contact and features a strong plastic deformation for the re-established contact - in contrast to the previous case of large particles.

The contact model by Luding [102] follows similar considerations as others, except for the fact that the mechanical contact does not detach, as discussed next. The irreversible, elastic re-loading before complete detachment can be seen as a compromise between small and large particle mechanics, i.e. between weak and strong attractive forces. It also could be interpreted as a premature re-establishment of mechanical contact, e.g., due to a rotation of the particles. Detachment and remaining non-contact is only then valid if the particles do not rotate relative to each other; in case of rotations, both sliding and rolling degrees of freedom can lead to a mechanical contact much earlier than in the ideal case of a perfect normal collision of ideal particles. In the spirit of a mesoscopic model, the irreversible contact model is due to the ensemble of possible contacts, where some behave like imagined in the ideal case, whereas some behave strongly different, e.g., due to some relative rotation. However, there are several other good reasons to consider an irreversible unloading branch.

In the case of asphalt (core-shell material with a stone core and bitumen-shell), dependent on the composition of the bitumen, it can contain a considerable amount of fine solids that will behave softly for loading, but rather stiff for re-loading (elastic $k_{2}$ branch). (Bitumen with fine fillers is referred to as "mastic", but a more detailed study of this class of materials goes beyond the scope of this study.) [136]

For atomistic nano-particles and for porous particles, one thing in common is the fact the typical deformations can be much larger than the primary particles inhomogeneities and that the adhesion of the primary particles is very strong, which leads to their re-arrangements (see Fig. 5 in Ref. [191]). That is the bulk material will deform plastically (irreversibly) even if the primary particles can be assumed to be perfectly elastic. We can not assume permanent ideal flattening and full loss of mechanical contact during unloading for the mesoscopic particles: Many contacts between the primary particles and surface inhomogeneities will be 


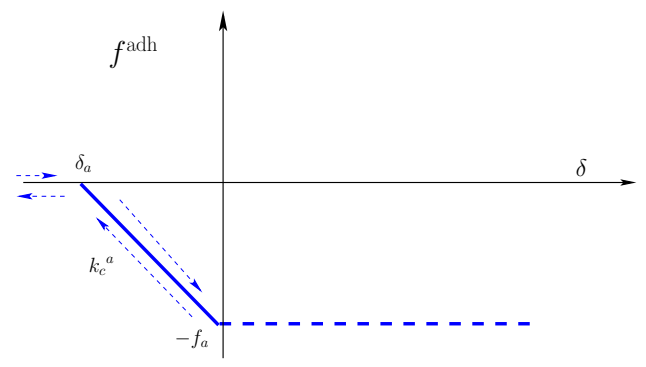

(a)

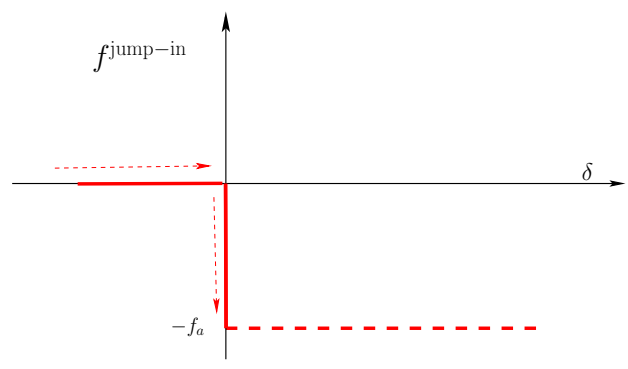

(b)

Figure 3.3: Schematic plots of (a) the non-contact adhesive force-overlap relation and (b) the non-contact jump-in force-overlap relation.

lost, but - due to their strong attraction - many others will keep their contacts. Strong clusters of primary particles will remain intact and can form bridges (threads) or clumps during unloading - which either keep the two surfaces in contact beyond the idealized detachment point or can lead to an elastic repulsion due to a clump-particle sitting between the surfaces (see Fig. 3 in Ref. [102]).

When re-loading, the (elastic) connecting elements influence the response; rearrangements of the primary particles (and clusters) happen on the surface, leading to its reshaping leaving a surely non-flat contact surface.

Also in this case, as often mentioned for granular systems, the interaction of several elastic particles does not imply bulk elasticity of the assembly, due to rearrangements. Thus, in the present model an irreversible tensile branch is assumed, without distinction between the behavior before and after the first contact-loss-point other than the intrinsic non-linearity in the model: The elastic stiffness for re-loading $k_{2}$ decreases the closer it comes to $\delta=0$; in the present version of the contact model, $k_{2}$ for unloading from the $k_{1}$ branch and for re-loading from the $k_{c}$ branch are exactly matched.

As a final remark, for almost all models on the market - due to convenience and numerical simplicity, in case of complete detachment $\delta<0$ - the contact is set to its initial state, since it is very unlikely that the two particles will touch again at exactly the same contact point as before. On the other hand in the present model a long-range interaction is introduced, in the same spirit as [201, 204]. Reloading in the non-contact $(\delta<0)$ regime is perfectly elastic, as it refers to the non-contact of surfaces and is due only to long-range attractive (van der Waals) forces, as will be detailed in the next subsection. 


\subsubsection{Non-contact normal force}

It has been shown in many studies that long-range interactions are present when dry adhesive particles collide, i.e. forces are present even for negative overlap $\delta$ [102, 197, 204, 205]. In the previous section, we have studied the force laws for contact overlap $\delta>0$. In this section we introduce a description for non-contact, long range, adhesive forces, focusing on the two non-contact models schematically shown in Fig. 3.3 - both piece-wise linear in the spirit of the mesoscopic model - namely the reversible model and the jump-in (irreversible) noncontact models (where the latter could be seen as an idealized, mesoscopic representation of a liquid bridge, just for completeness). Later, in the next section, we will combine noncontact and contact forces.

\subsubsection{Reversible Adhesive force}

In Fig. 3.3(a) we consider the reversible attractive case, where a (linear) van der Waals type long-range adhesive force is assumed. The force law can be written as

$$
f^{\mathrm{adh}}= \begin{cases}-f_{a} & \text { if } \delta>0 \\ -k_{c}^{a} \delta-f_{a} & \text { if } 0 \geq \delta>\delta_{a} \\ 0 & \text { if } \delta_{a}>\delta\end{cases}
$$

with the range of interaction $\delta_{a}=-f_{a} / k_{c}^{a}$, where $k_{c}^{a}>0$ is the adhesive strength of the material and $f_{a}>0$ is the (constant) adhesive force magnitude, active for overlap $\delta>0$ in addition to the contact force. When $\delta=0$ the force is $-f_{a}$. The adhesive force $f^{\text {adh }}$ is active when particles are closer than $\delta_{a}$, when it starts increase/decrease linearly along $-k_{c}^{a}$, for approach/separation respectively. In the rest of the chapter, for the sake of simplicity and without loss of generality, the adhesive strength will be chosen as coincident with the contact adhesive stiffness in Sec. 3.2.2.2, that is $k_{c}^{a}=k_{c}$.

\subsubsection{Jump-in (Irreversible) Adhesive force}

In Fig. 3.3(b) we report the behavior of the non-contact force versus overlap when the approach between particles is described by a discontinuous (irreversible) attractive law. The jump-in force can be simply written as

$$
f^{\text {jump-in }}=\left\{\begin{array}{ll}
0 & \text { if } \delta<0 \\
-f_{a} & \text { if } \delta \geq 0
\end{array} .\right.
$$

As suggested in previous studies [23, 78, 197], there is no attractive force before the particles come into contact; the adhesive force becomes active and suddenly drops to a negative value, $-f_{a}$, at contact, when $\delta=0$. The jump-in force resembles the limit case $k_{c}^{a} \rightarrow \infty$ of Eq. (3.15). Note that the behavior is defined here only for approach of the particles. We assume 
the model to be irreversible, as in the unloading stage, during separation, the particles will not follow this same path (details will be discussed below).

\subsection{Coefficient of Restitution}

As already mentioned, we can quantify the amount of dissipated energy relative to the incident kinetic energy in terms of the coefficient of restitution $e$, by using the expression $1-e^{2}$.

When we consider a pair collision, with particles approaching from infinite distance, the coefficient of restitution can be defined as

$$
e=\frac{v_{f}^{\infty}}{v_{i}^{\infty}}
$$

and further decomposed as

$$
e=\frac{v_{f}^{\infty}}{v_{f}} \frac{v_{f}}{v_{i}} \frac{v_{i}}{v_{i}^{\infty}}=\epsilon_{o} e_{n} \epsilon_{i},
$$

where three different regimes have been introduced to describe the pair interaction. $\epsilon_{i}$ and $\epsilon_{o}$ are the pull-in and pull-off coefficients of restitution, that describe the non-contact parts of the interaction $(\delta<0)$, for approach and separation of particles respectively, while $e_{n}$ is the coefficient of restitution defined for particle in contact $(\delta>0) . v_{i}^{\infty}$ and $v_{f}^{\infty}$ denote the approach and separation velocities at infinity distance, when the (short- and long-range) interaction force is zero. $v_{i}$ is the approaching velocity at zero contact overlap $\delta=0$ (start of contact) and $v_{f}$ is the separation velocity at zero overlap $\delta=0$ when the particles are separating (end of contact).

In the following, we will first analyze each term in Eq. (3.17b) separately, based on energy considerations. Then we will show combined contact models using the non-contact and contact components described in sections 3.2.2-3.2.3 and provide the coefficient of restitution for this wide class of models.

\subsubsection{Pull-in coefficient of restitution}

In order to describe the pull-in coefficient of restitution $\epsilon_{i}$ we focus on the two non-contact models proposed in Sec. 3.2.3, as simple interpretations of the adhesive force during the approach of the particles.

When the reversible adhesive contact model is used, the energy conservation argument

$$
\frac{1}{2} m_{r} v_{i}^{\infty 2}=\frac{1}{2} f_{a} \delta_{a}+\frac{1}{2} m_{r} v_{i}^{2}
$$

yields the following expression for $\epsilon_{i}$ :

$$
\epsilon_{i}^{\mathrm{adh}}=\frac{v_{i}}{v_{i}^{\infty}}=\sqrt{1-\frac{f_{a} \delta_{a}}{m_{r} v_{i}^{\infty}}}=\sqrt{1+\frac{f_{a}^{2} / k_{c}}{m_{r} v_{i}^{\infty 2}}} .
$$


The pull-in coefficient of restitution increases with increasing adhesive force magnitude $f_{a}$ due to increase in attractive force and decreases with the adhesive strength of the material $k_{c}$. On the other hand, if the irreversible adhesive jump-in model is implemented, a constant value $\epsilon_{i}^{\text {jump-in }}=1$ is obtained, as $f^{\text {jump-in }}=0$ for $\delta<0$ and the velocity is constant $v_{i}=v_{i}^{\infty}$.

\subsubsection{Normal coefficient of restitution}

When focusing on the contact coefficient of restitution $e_{n}$ and writing the energy balance between the start and end of contact interaction, we get

$$
\frac{1}{2} m_{r} v_{i}^{2}=W_{\text {diss }}+\frac{1}{2} m_{r} v_{f}^{2}
$$

and

$$
e_{n}=\frac{v_{f}}{v_{i}}=\sqrt{1-\frac{2 W_{d i s s}}{m_{r} v_{i}^{2}}},
$$

where $W_{\text {diss }}$ denotes the amount of energy dissipated during the collision.

If the linear contact model (see Sec. 3.2.2.1) is considered in the absence of viscous damping (LS), $W_{\text {diss }}$ is zero, hence the normal coefficient of restitution $e_{n}^{\mathrm{LS}}=1$. On the other hand, for either viscous damping or in the case of adhesive elasto-plastic contacts (see Sec 3.2.2.2), there is finite dissipation. As $W_{\text {diss }}$ is always positive, the normal coefficient of restitution is always smaller than unity, i.e. $e^{\mathrm{LSD}}<1$ and $e_{n}^{\mathrm{HYS}}<1$. The coefficient of restitution for the linear spring dashpot model is given in Eq. (3.5), while the elasto-plastic contact model will be discussed below.

\subsubsection{Pull-off coefficient of restitution}

The pull-off coefficient of restitution is defined for particles that lose contact and separate, using the adhesive reversible model as described in section 3.2.3.1.

By assuming energy conservation

$$
\frac{1}{2} m v_{f}^{\infty 2}=\frac{1}{2} f_{a} \delta_{a}+\frac{1}{2} m v_{f}^{2},
$$

we obtain the following expression

$$
\epsilon_{o}=\frac{v_{f}^{\infty}}{v_{f}}=\sqrt{1+\frac{f_{a} \delta_{a}}{m v_{f}^{2}}}=\sqrt{1-\frac{f_{a}^{2} / k_{c}}{m v_{f}^{2}}} .
$$

Similarly to what already seen for Eq. 3.18b, the pull-off coefficient of restitution depends on both the adhesive force magnitude $f_{a}$ and strength $k_{c}$, other than the separation velocity 


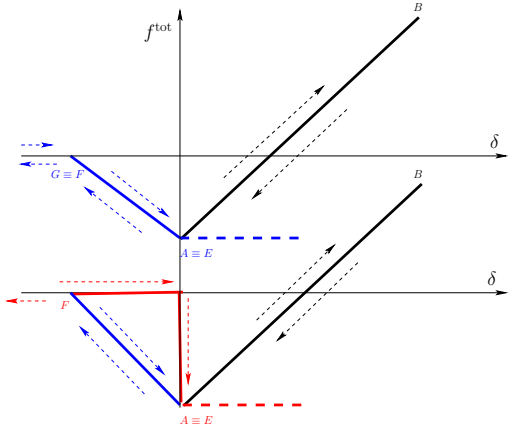

(a)

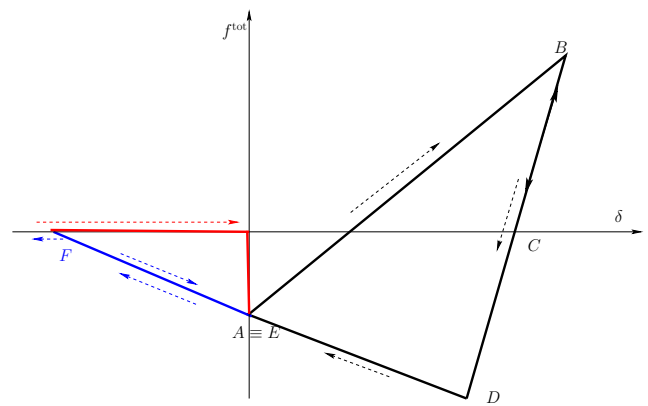

(b)

Figure 3.4: (a) Reversible and irreversible non-contact forces, where the top blue line (for negative overlap) represents the former and the bottom red line (for negative overlap) the latter. The black line for positive overlap represents the linear contact force as superimposed on the non-contact force. (b) Force-displacement law for elasto-plastic, adhesive contacts superimposed on the irreversible non-contact adhesive force.

$v_{f}$. As the particles feel an attractive force during unloading, part of their kinetic energy is lost and hence $\epsilon_{o}<1$ in Eq. (3.20b).

It is worthwhile to notice that the force-overlap picture described above, with $\epsilon_{o}$ defined as in Eq. (3.20b) refers to a system with sufficiently high impact velocity, so that the particles can separate with a finite kinetic energy at the end of collision. That is

$$
v_{f}^{2}>f_{a}^{2} /\left(m k_{c}\right)=:\left(v_{f}^{a}\right)^{2}
$$

or, equivalently, $v_{i}^{\infty}>v_{f}^{a} /\left(e_{n} \epsilon_{i}\right)$, where $v_{f}^{a}$ denotes the maximum relative velocity at which particles actually can separate. On the other hand, if the kinetic energy reaches zero before the separation, e.g. during the unloading path, the particles start re-loading along the adhesive branch until the value $\delta=0$ is reached and they follow contact law defined for $\delta>0$ again.

\subsubsection{Combined contact laws}

The contact and non-contact models described in previous sections 3.3.1, 3.3.2 and 3.3.3 can be combined in order to obtain the overall description of the system behavior, during approach, contact and final separation of the particles.

For example, the combination of the pull-in, the linear normal and the pull-off components leads to a reversible adhesive linear contact model, as shown schematically in the upper part of Fig. 3.4a, with coefficient of restitution $e=\epsilon_{o} e_{n}^{\mathrm{LSD}} \epsilon_{i}^{\mathrm{adh}}$. On the other hand, by combining the irreversible (jump-in) pull-in, the linear normal and the (reversible) pull-off 


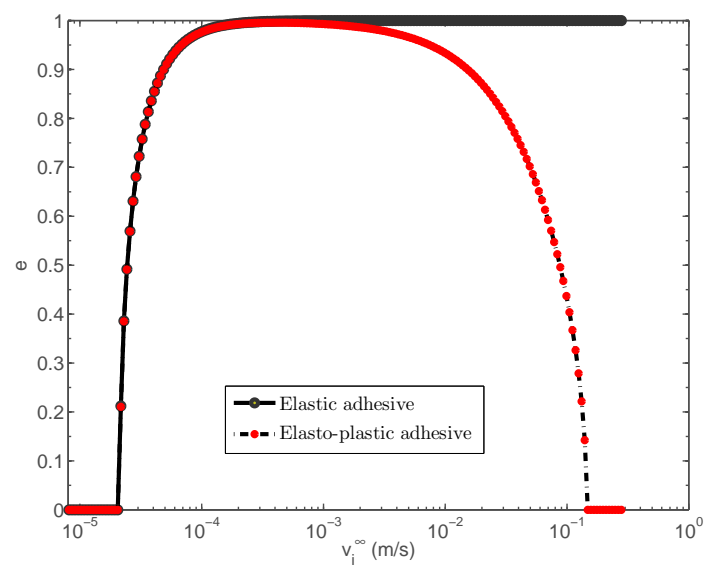

Figure 3.5: Restitution coefficient $e$ plotted as a function of the impact velocity $v_{i}^{\infty} e_{n} \rightarrow e$ and $v_{i} \rightarrow v_{i}^{\infty}$ for irreversible elastic-adhesive and elasto-plastic adhesive spheres (as given in the inset). Parameters used here are $k_{1}=10^{2} \mathrm{Nm}^{-1}, k_{p}=5 \times 10^{2} \mathrm{Nm}^{-1}, k_{c}=10^{2} \mathrm{Nm}^{-1}$, and $f_{a}=5 \times 10^{-7} \mathrm{~N}$, which leads to the low-velocity sticking limit $v_{f}^{a}=2.1 \times 10^{-5} \mathrm{~m} / \mathrm{s}$, for particles with radius $1.1 .10^{-3} \mathrm{~m}$, density $2000 \mathrm{~kg} / \mathrm{m}^{3}$, and mass $m=5.6 \times 10^{-6} \mathrm{~kg}$.

components (see schematic in the lower part of Fig. 3.4a) we get coefficient of restitution $e=\epsilon_{o} e_{n}^{\mathrm{LSD}} \epsilon_{i}^{\text {jump-in }}$.

In the following we will focus on the combination of the irreversible pull-in with the adhesive elasto-plastic and the (reversible) pull-off parts, leading to an irreversible adhesive elastoplastic model, see Fig. 3.4b, with $e=\epsilon_{o} e_{n}^{\mathrm{HYS}} \epsilon_{i}^{\mathrm{jump}-\mathrm{in}}=\epsilon_{o} e_{n}^{\mathrm{HYS}}$. For this special case we want to analyze the influence of the adhesive component/parameters on the overall behavior.

In Fig. 3.5, we plot the coefficient of restitution $e$ as a function of impact velocity for both the irreversible elastic $\left(e_{n}=e_{n}^{\mathrm{LSD}}\right)$ and the irreversible elasto-plastic $\left(e_{n}=e_{n}^{\mathrm{HYS}}\right)$ contact models. We observe that for low velocity the system behaves in a similar fashion in both cases, showing an initial sticking regime, in agreement with previous experimental and numerical results [186, 197, 217]. At higher velocities, a significant difference appears: for elastic adhesive spheres, $e$ keeps increasing and approaches unity while, for elasto-plastic adhesive spheres, $e$ starts decreasing at intermediate velocity until it becomes zero at higher velocity. This difference is related with the sources of dissipation in the two models. In the irreversible elastic case, energy is dissipated only due to the pull-off of the particles, which is significant in low velocity range only. On the contrary, for irreversible elasto-plastic spheres, dissipation takes place during both, pull-off and normal contact, stages. The latter, new, effect is negligible for low velocity (hence the two models coincide) but it becomes important for large impact velocity, leading to a second, high velocity, sticking regime, (that will be discussed in detail below). 


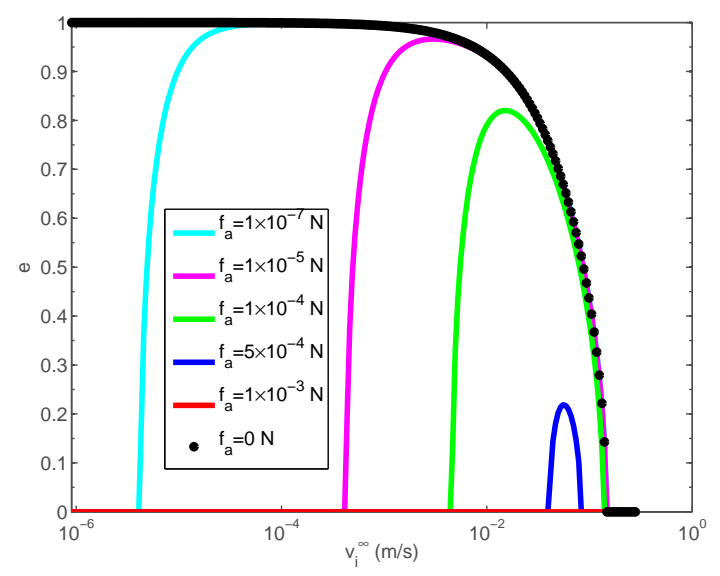

Figure 3.6: Restitution coefficient plotted as function of impact velocity without viscous dissipation. Different lines correspond to the analytical expression in Eq. (3.28) with different non-contact adhesion $f_{a}$ as given in the inset. Black circles represent the numerical solution results for $f_{a}=0$, where all other parameters are the same as in Fig. 3.5.

Furthermore, in Fig. 3.6, we focus on the strength of the non-contact adhesion $f_{a}$ and we plot $e$ against the impact velocity for different $f_{a}$. We observe that for $f_{a}=0, e_{n} \approx 1$ at low velocities, while, for finite $f_{a}$, the particles stick to each other with $e_{n}=0$. The critical velocity $v_{f}^{a}$ required to separate the particles increases with $f_{a}$. For extreme values of $f_{a}$ the particles stick in the whole range of velocities.

It is interesting to notice that for very low $f_{a}$ and low impact velocities the behavior is independent of the adhesive force magnitude (cyan line and black circles lie on top of each other in Fig. 3.6). In the further sections we restrict our analysis to this range of $f_{a}$ and impact velocity.

\subsection{Elasto-plastic coefficient of restitution}

In the following we will restrict our analytical study on the coefficient of restitution to the range of moderate and large impact velocity, where the contribution of weak non-contact adhesive forces $f_{a} \rightarrow 0$ can be neglected. Furthermore, we disregard viscous forces in order to allow for a closed analytical treatment. The coefficient of restitution will be computed and its dependence on the impact velocity $v_{i}$ and the adhesive stiffness $k_{c}$ is considered for two cases $v_{i}<v_{p}$ and $v_{i} \geqslant v_{p}$, with $v_{p}$ defined in Eq. (3.14). 


\subsubsection{Theory and dimensionless parameters}

\subsubsection{Initial relative velocity $v_{i}<v_{p}$}

When $v_{i}<v_{p}$ the particles after loading, unload with slope $k_{2}$ and the system deforms along the path $0 \rightarrow \delta_{\max } \rightarrow \delta_{0} \rightarrow \delta_{\min } \rightarrow 0$, as described in the Sec. 3.2.2.2 and shown in Fig. 3.2.

The initial kinetic energy (at $\delta=0$ overlap) is completely transformed to potential energy at the maximum overlap $\delta_{\max }$,

$$
\frac{1}{2} m_{r} v_{i}^{2}=\frac{1}{2} k_{1} \delta_{\max }^{2}
$$

The direction of relative velocity is reversed at $\delta_{\max }$, unloading starts with slope $k_{2}$ and some part of the potential energy is converted to kinetic energy at the force-free overlap $\delta_{0}$,

$$
\frac{1}{2} m_{r} v_{0}^{2}=\frac{1}{2} k_{2}\left(\delta_{\max }-\delta_{0}\right)^{2},
$$

which, using Eq. (3.8), can be written as

$$
\frac{1}{2} m_{r} v_{0}^{2}=\frac{1}{2} k_{1} \delta_{\max }\left(\delta_{\max }-\delta_{0}\right) \text {. }
$$

Further unloading, below $\delta_{0}$, leads to attractive forces. The kinetic energy at $\delta_{0}$ is partly converted to potential energy at $\delta_{\min }$

$$
\frac{1}{2} m_{r} v_{\min }^{2}+\frac{1}{2} k_{2}\left(\delta_{\min }-\delta_{0}\right)^{2}=\frac{1}{2} m_{r} v_{0}^{2} .
$$

The total energy is finally converted to only kinetic energy at the end of the collision (overlap $\delta=0)$

$$
\frac{1}{2} m_{r} v_{f}^{2}-\frac{1}{2} m_{r} v_{\min }^{2}=-\frac{1}{2} k_{c} \delta_{\min }^{2},
$$

that, when combined with $(3.22 \mathrm{~d})$, gives

$$
\frac{1}{2} m_{r} v_{f}^{2}-\frac{1}{2} m_{r} v_{0}^{2}=-\frac{1}{2} k_{c} \delta_{\min } \delta_{0}
$$

Using Eqs. (3.22a), (3.22c), and (3.22f) with the definitions of $\delta_{\min }$ and $\delta_{0}$, and dividing by the initial kinetic energy, we obtain the coefficient of restitution

$$
e_{n}^{(1)}=\frac{v_{f}}{v_{i}}=\sqrt{\frac{k_{1}}{k_{2}}-\frac{k_{c}}{k_{1}} \frac{\left(k_{2}-k_{1}\right)}{\left(k_{2}+k_{c}\right)} \frac{\left(k_{2}-k_{1}\right)}{k_{2}}}
$$

with $k_{2}=k_{2}\left(\delta_{\max }\right)=k_{2}\left(v_{i}\right)$, as defined in Eq. (5.9). 


\subsubsection{Initial relative velocity $v_{i} \geqslant v_{p}$}

When the initial relative velocity $v_{i}$ is large enough such that $v_{i} \geqslant v_{p}$, the estimated maximum overlap $\delta_{\max }=v_{i} \sqrt{m_{r} / k_{1}}$ is greater than $\delta_{\max }^{p}$. Let $v_{1}$ be the velocity at overlap $\delta_{\max }^{p}$. The system deforms along the path $0 \rightarrow \delta_{\max }^{p} \rightarrow \delta_{\max } \rightarrow \delta_{0} \rightarrow \delta_{\min } \rightarrow 0$, see Fig. 3.2. The initial relative kinetic energy is not completely converted to potential energy at $\delta=\delta_{\max }^{p}$, hence

$$
\frac{1}{2} m_{r} v_{i}^{2}=\frac{1}{2} m_{r} v_{1}^{2}+\frac{1}{2} k_{1}\left(\delta_{\max }^{p}\right)^{2}
$$

and the loading continues with the slope $k_{p}$ until all kinetic energy equals zero at $\delta=\delta_{\max }>$ $\delta_{\max }^{p}$

$$
\frac{1}{2} m_{r} v_{1}^{2}+\frac{1}{2} k_{1}\left(\delta_{\max }^{p}\right)^{2}=\frac{1}{2} k_{p}\left(\delta_{\max }-\delta_{0}\right)^{2},
$$

the relative velocity changes sign at $\delta_{\max }$, the contact starts to unload with slope $k_{p}$. The potential energy is completely converted to kinetic energy at $\delta_{0}$, such that the equality

$$
\frac{1}{2} m_{r} v_{0}^{2}=\frac{1}{2} k_{p}\left(\delta_{\max }-\delta_{0}\right)^{2}
$$

or

$$
\frac{1}{2} m_{r} v_{0}^{2}=\frac{1}{2} k_{1} \delta_{\max }^{p}\left(\delta_{\max }^{p}-\delta_{0}\right)+\frac{1}{2} m_{r} v_{1}^{2}
$$

holds. Further unloading, still with slope $k_{p}$, leads to attractive forces until $\delta_{\min }^{p}$ is reached, where the kinetic energy is partly converted to potential energy

$$
\frac{1}{2} m_{r} v_{\min }^{2}+\frac{1}{2} k_{p}\left(\delta_{\min }^{p}-\delta_{0}\right)^{2}=\frac{1}{2} m_{r} v_{0}^{2} .
$$

The unloading continues along $k_{c}$ and the total energy at $\delta_{\min }^{p}$ is finally converted to only kinetic energy at the end of collision ( $\delta=0$ overlap), so that

$$
\frac{1}{2} m_{r} v_{f}^{2}-\frac{1}{2} m_{r} v_{\min }^{2}=-\frac{1}{2} k_{c}\left(\delta_{\min }^{p}\right)^{2} .
$$

Using Eqs. (3.24c) and (3.24d) in Eq. (3.24f) gives

$$
\frac{1}{2} m_{r} v_{f}^{2}-\frac{1}{2} m_{r} v_{0}^{2}=-\frac{1}{2} k_{c} \delta_{\min }^{p} \delta_{0}
$$

Combining Eqs. (3.24a), (3.24b), (3.24c), (3.24g) with the definitions of $\delta_{\min }^{p}$ and $\delta_{0}$, and dividing by the initial kinetic energy, we obtain the coefficient of restitution

$$
e_{n}^{(2)}=\sqrt{1+\left[-1+\frac{k_{1}}{k_{p}}-\frac{k_{c}}{k_{1}} \frac{\left(k_{p}-k_{1}\right)^{2}}{\left(k_{p}+k_{c}\right) k_{p}}\right] \frac{v_{p}^{2}}{v_{i}^{2}}}
$$

with $v_{p} / v_{i}<1$. 


\subsubsection{Dimensionless Parameters}

For a more general description, a few dimensionless parameters can be defined:

$$
\begin{aligned}
& \text { Plasticity }: \eta=\frac{k_{p}-k_{1}}{k_{1}}, \\
& \text { Adhesivity : } \beta=\frac{k_{c}}{k_{1}}, \\
& \text { Scaled initial velocity }: \chi=\frac{\delta_{\max }}{\delta_{\max }^{p}} \sim \frac{v_{i}}{v_{p}} .
\end{aligned}
$$

The final dimensionless number, given here for the sake of completeness, but not used in this subsection, is the ratio of maximum velocity at which particles stick due to adhesion only to the initial relative velocity of the particles. $\psi_{a}=v_{a} / v_{i}^{\infty} \ll 1$.

Using Eqs. (3.26a), (3.26b) and (3.26c) in Eq. (5.9), one can define

$$
k_{2}(\chi)=\left\{\begin{array}{ll}
k_{p} & \text { if } \chi \geq 1 \\
k_{1}(1+\eta \chi) & \text { if } \chi<1
\end{array},\right.
$$

while the coefficients of restitution, $e_{n}^{(1)}$ in Eq. (3.23) and $e_{n}^{(2)}$ in Eq. (3.25) become

$$
e_{n}^{(1)}(\eta, \beta, \chi<1)=\sqrt{\frac{1}{1+\eta \chi}-\frac{\beta \eta^{2} \chi^{2}}{(1+\eta \chi)(1+\beta+\eta \chi)}}
$$

and

$$
e_{n}^{(2)}(\eta, \beta, \chi \geq 1)=\sqrt{1+[A(\eta, \beta)-1] \frac{1}{\chi^{2}}},
$$

with

$$
A(\eta, \beta)=\left[e_{n}^{(1)}(\eta, \beta, \chi=1)\right]^{2}
$$

\subsubsection{Qualitative Description}

In Fig. 3.7, the analytical prediction for the coefficient of restitution, from Eqs. (3.28) and (3.29), is compared to the numerical integration of the contact model, for different scaled initial velocities $\chi$. We confirm the validity of the theoretical prediction for the coefficient of restitution in the whole range.

For very small $\eta \chi<10^{-3}, e_{n}$ can be approximated as $e_{n}^{(1)} \approx 1-\frac{\eta \chi}{2}$, which gives a good agreement to 2 orders of magnitude in velocity. With increasing initial relative velocity $v_{i}$, 


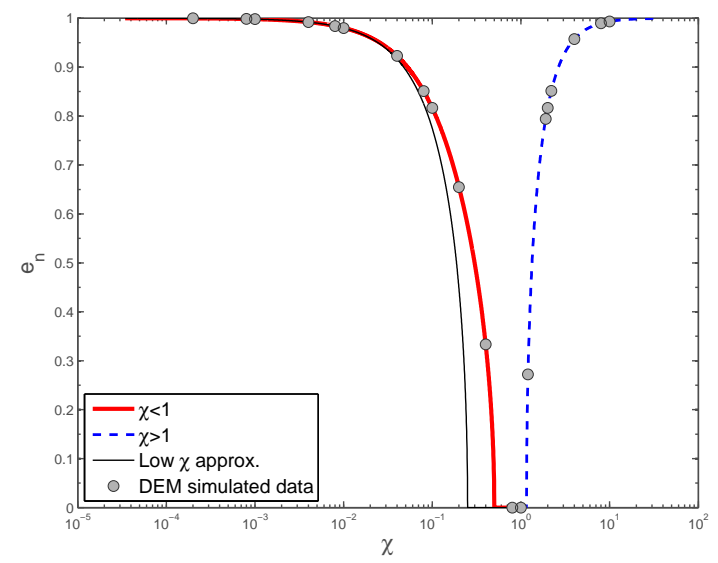

Figure 3.7: Restitution coefficient plotted as a function of the scaled initial velocity $\chi$ for a collision without viscous and non-contact forces. The thick red line correspond to the analytical expressions in Eq. (3.28), the dashed blue line to Eq. (3.29), and circles are DEM simulations, while the thin black line represents the low velocity approximation results for the same material parameters as in Fig. 3.5, i.e. $\eta=4$ and $\beta=1$.

dissipation increases faster than the initial kinetic energy leading to a faster convex decrease of $e_{n}^{(1)}$. The coefficient of restitution $e_{n}^{(1)}$ becomes zero when a critical scaled initial velocity $\chi_{c}^{(1)}$ (see Eq. (3.32)) is reached. At this point, the amount of dissipated energy is equal to the initial kinetic energy, leading the particles to stick. The coefficient of restitution remains zero until a second critical scaled initial velocity $\chi_{c}^{(2)}$ is reached, i.e. sticking behavior is observed for $\chi_{c}^{(1)} \leq \chi \leq \chi_{c}^{(2)}$. Finally, for $\chi>\chi_{c}^{(2)}$, the dissipated energy remains constant (plastic limit is reached), while the initial kinetic energy increases. As a result, the kinetic energy after collision increases and so does the coefficient of restitution $e_{n}$. Existence of sticking at such high velocities is recently reported by Kothe et al. [86], where authors studied the outcome of collisions between sub-mm-sized dust agglomerates in micro-gravity. ${ }^{1}$ The increase in $e_{n}$ with the velocity is because of a limit on maximum plastic overlap, thereby making the plastic dissipation to be constant for $\delta>\delta_{\max }^{p}$.

Certainly, this is in contrast to what is expected and observed in experiments on some types of elasto-plastic granular materials. We point out that this behavior shows up only at very high velocities (x-axis in Fig. 3.7 has increased by 2 orders of magnitude). Moreover, the definition of $e_{n}$ might become questionable at that high velocity, as the particles can fragment/break and physics of the system has to be reconsidered. At such large deformations the particles cannot be assumed to be spherical anymore. On the other hand, the increase of

1. Note that this is the regime where the physics of the contact changes and dependent on the material and other considerations, modifications to the contact model could/should be applied, however, this goes beyond the scope of this study. 


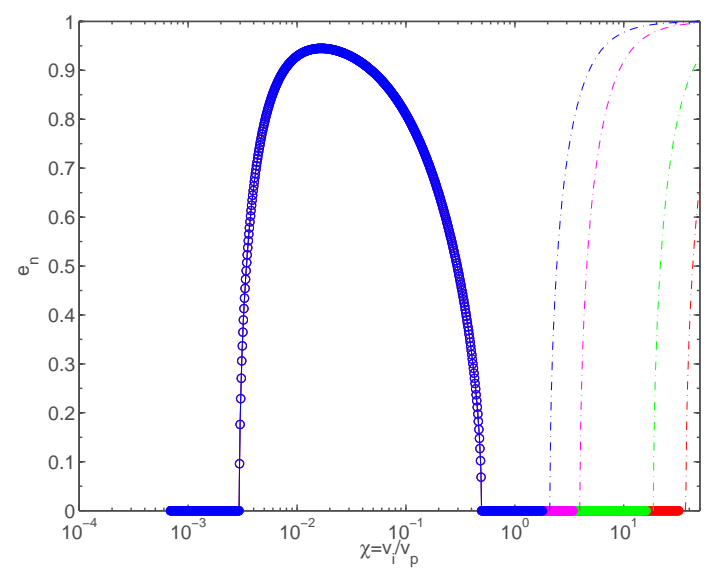

Figure 3.8: Restitution coefficient plotted as a function of the scaled initial velocity $\chi$. The blue solid line corresponds to the solution for $\chi<1$, with $\phi_{f}=0.05$, while the dashed lines with different colors represent the behavior once limit overlap is reached. Magenta, green and red represent $e_{n}$ for $\phi_{f}{ }^{\prime}=0.1, \phi_{f}{ }^{\prime}=0.5$ and $\phi_{f}{ }^{\prime}=1.0$ respectively, where the latter is the fully plastic case, as if one would set $\delta_{0}^{p}=a$, where $a$ is the particle radius. Note that $k_{p}$ is adapted as described in appendix 3.C.

$e_{n}$ for high velocity is a familiar observation in the cold spray community $[168,189,232]$. After a given (critical) velocity the spray particles adhere to the substrate, and they do so for a range of impact velocities, while increasing velocity further leads to unsuccessful deposition, i.e. the particles will bounce from the substrate. The sticking phenomenon of the material has been extensively studied experimentally and numerically [168, 189, 228, 229, 232].

As explained in the previous section 3.2.2.3, a pre-determinate threshold $\delta^{p}$ max on the plastic regime is the reason for the anomalous elastic high velocity behavior of the coefficient of restitution. The model can be tuned by choosing a higher $\phi_{f}$, re-calculating $k_{p}$, thereby keeping the behavior plastic in the whole range of interest for the velocity and the collision dynamics up to $\delta_{\max }^{p}$ unchanged (see appendix 3.C). Nevertheless, we consider a reference value for $\phi_{f}$ (and $k_{p}$ ) based on the maximum volume fraction of the multi-particle granular assembly, such that the macroscopic behavior is reasonable and in good agreement with the experiment, e.g. based on our simulations on split-bottom ring shear cell [107]. Also, in a typical DEM simulation, the maximum overlap is $5-10 \%$ of the reduced particle radius. This is illustrated in Fig. 3.8, where we plot $e_{n}$ against the normalized velocity $\chi=\frac{v_{i}}{v_{p}}$, for different values of $\phi_{f}$, with $v_{p}$ calculated from the same reference stiffness input parameters $\phi_{f}$ and $k_{p}$. Reference $\phi_{f}$ is chosen to be 0.05 , which would mean $\delta_{0}^{p}=0.05 a$. For low velocity the different curves lie on the top of each other, showing that the collision dynamics stays unaffected by the change in $\phi_{f}$. The onset of the increase in $e_{n}$ shifts to right with increasing $\phi_{f}$, providing a fully plastic regime at high $\chi$ if desired. 


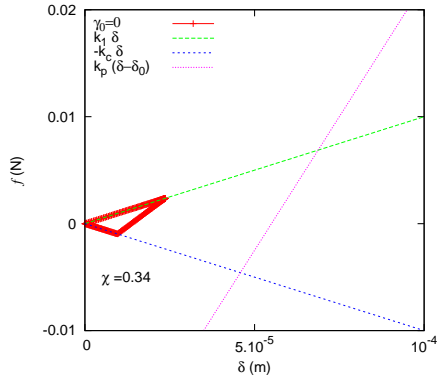

(a)

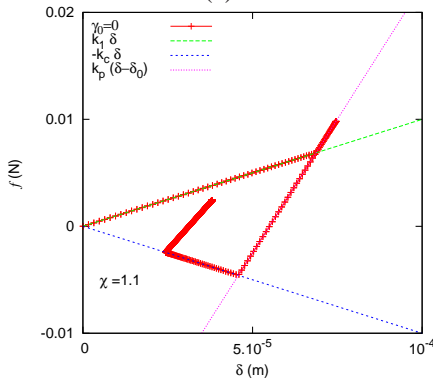

(c)

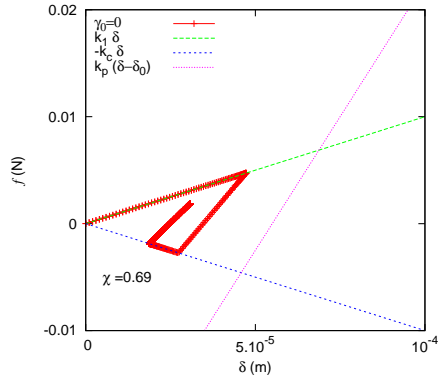

(b)

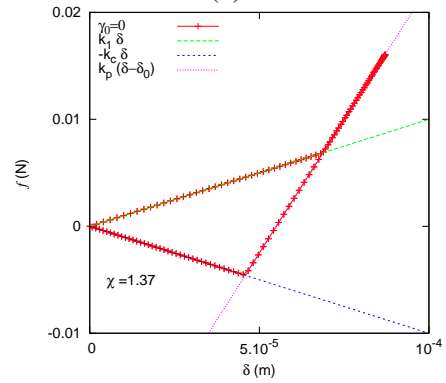

(d)

Figure 3.9: Contact force during one collision, plotted against the overlap for different scaled initial velocities $\chi=0.34,0.69,1.1$, and 1.37 , respectively. The three straight lines represent the plastic branch, with slope $k_{1}$, the adhesive branch, with slope $-k_{c}$, and the limit branch with slope $k_{p}$, for $k_{1}=10^{2} \mathrm{Nm}^{-1}, k_{p}=5 \times 10^{2} \mathrm{Nm}^{-1}, k_{c}=10^{2} \mathrm{Nm}^{-1}$ and $\phi_{f}=0.05$, i.e. $\eta=4$ and $\beta=1$

In Fig. 3.9, we compare the variation of the force with overlap in the various regimes of $\chi$ as discussed above for $\phi_{f}=0.05$. For very small $\chi$, the unloading slope $k_{2} \approx k_{1}$, (see Fig. 3.9a for a moderately small $\chi=0.34$ ), and the amount of dissipated energy is small, increasing with $\chi$. The kinetic energy after collision is almost equal to the initial kinetic energy, i.e. $e_{n} \sim 1$, see Fig. 3.7. In Figs. 3.9b and 3.9c, the force-overlap variation is shown for sticking particles, for the cases $\chi_{c}^{(1)}<\chi<1$ and $1<\chi<\chi_{c}^{(2)}$, respectively (more details will be given in the following subsection). Finally, in Fig. 3.9d, the case $\chi>\chi_{c}^{(2)}$ is displayed, for which the initial kinetic energy is larger than the dissipation, resulting in the separation of the particles. The corresponding energy variation is described in detail in the appendix.

\subsubsection{Sticking regime limits and overlaps}

In this section we focus on the range of $\chi_{c}^{(1)}<\chi \leq \chi_{c}^{(2)}$, where the particles stick to each other and calculate the critical values $\chi_{c}^{(1)}$ and $\chi_{c}^{(2)}$. Also we assume $\beta$ to be large enough so that sticking is possible, as we show in later section that for a given $\eta$ a minimum $\beta=\beta^{*}$ 
is required for particles to stick. When $\chi=\chi_{c}^{(1)}$ all initial kinetic energy of the particles is dissipated during the collision. Hence the particles stick and $e_{n}$ becomes zero:

$$
e_{n}^{(1)}\left(\eta, \beta, \chi_{c}^{(1)}\right)=0
$$

which leads to

$$
\beta \eta^{2} \chi^{2}-\eta \chi-(1+\beta)=0
$$

Only the positive $\chi$ solution is physically possible, as particles with negative initial relative velocity cannot collide, so that

$$
\chi_{c}^{(1)}=\frac{1}{2 \beta \eta}[1+\sqrt{1+4 \beta(1+\beta)}] .
$$

For larger $\chi>\chi_{c}^{(1)}$, the dissipation is strong enough to consume all the initial kinetic energy, hence the particles loose kinetic energy at a positive, finite overlap $\delta_{\mathrm{c}}$, see Fig. 3.9b. The contact deforms along the path $0 \rightarrow \delta_{\max } \rightarrow \delta_{0} \rightarrow \delta_{\min } \rightarrow \delta_{\mathrm{c}}$. Thereafter, in the absence of other sources of dissipation, particles keep oscillating along the same slope $k_{2}$. In order to compute $\delta_{\mathrm{c}}$, we use the energy balance relations in Eqs. (3.22), and conservation of energy along $\delta_{\min } \rightarrow \delta_{\mathrm{c}}$, as described by Eq. (3.22e)

$$
\frac{1}{2} m_{r} v_{f}^{2}-\frac{1}{2} m_{r} v_{0}^{2}=-\frac{1}{2} k_{c}\left\{\delta_{\min } \delta_{0}-\frac{1}{2} k_{c} \delta_{\mathrm{c}}^{2}\right\},
$$

with $v_{f}=0$ at the overlap $\delta_{c}$. Re-writing in terms of $k_{c}$ and $\delta_{\max }$ leads to

$$
k_{c} \delta_{\mathrm{c}}^{2}+\left\{\frac{k_{1}^{2}}{k_{2}}-\frac{k_{c}\left(k_{2}-k_{1}\right)^{2}}{k_{2}\left(k_{2}+k_{c}\right)}\right\} \delta_{\max }^{2}=0
$$

and thus to the sticking overlap

$$
\frac{\delta_{\mathrm{c}}^{(1)}}{\delta_{\max }^{p}}=\frac{\delta_{\max }}{\delta_{\max }^{p}} \sqrt{\frac{\left(k_{2}-k_{1}\right)^{2}}{k_{2}\left(k_{2}+k_{c}\right)}-\frac{k_{1}^{2}}{k_{2} k_{c}}} .
$$

In terms of dimensionless parameters, as defined earlier, one gets

$$
\frac{\delta_{\mathrm{c}}^{(1)}}{\delta_{\max }^{p}}=\chi \sqrt{\frac{\eta^{2} \chi^{2}}{(1+\eta \chi)(1+\beta+\eta \chi)}-\frac{1}{\beta(1+\eta \chi)}}=\frac{\chi}{\sqrt{\beta}}\left|e_{n}^{(1)}\right|,
$$

where $\left|e_{n}^{(1)}\right|$ denotes the absolute value of the result from Eq. (3.28).

For larger initial relative velocities, $\chi \geq 1$, the coefficient of restitution is given by Eq. (3.29), so that the second critical $1<\chi_{c}^{(2)}$ can be computed setting

$$
e_{n}^{(2)}\left(\eta, \beta, \chi_{c}^{(2)}\right)=0
$$




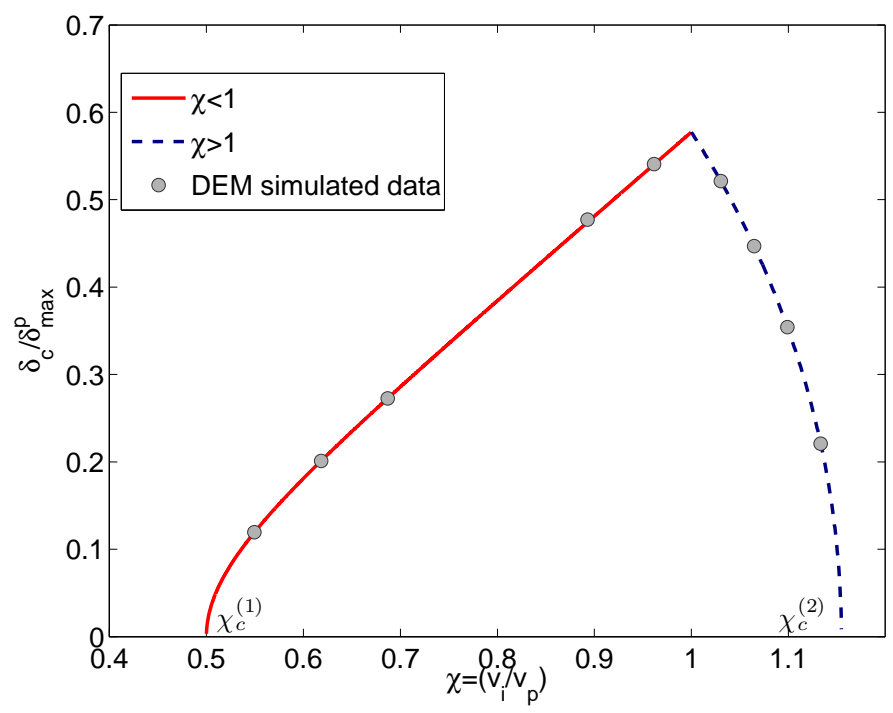

Figure 3.10: Kinetic energy-free contact overlap $\delta_{\mathrm{c}}$ plotted as a function of the scaled initial velocity $\chi=\frac{v_{i}}{v_{p}}$; the increasing branch corresponds to $\chi<1$, while the decreasing branch corresponds to $\chi>1$. The dots are simulations for $\eta=4$ and $\beta=1$, as in Fig. 3.9, which yields $\delta_{\mathrm{c}}^{\max } / \delta_{\max }^{p}=(1 / 3)^{1 / 2}$ in Eq. (3.39).

or

$$
\left[\frac{1}{1+\eta}-\frac{\beta \eta^{2}}{(1+\eta)(1+\beta+\eta)}-1\right] \frac{1}{\chi^{2}}=1 .
$$

Again, only the positive solution is physically possible, so that

$$
\chi_{c}^{(2)}=\sqrt{1-\frac{1}{1+\eta}+\frac{\beta \eta^{2}}{(1+\eta)(1+\beta+\eta)}}
$$

is the maximum value of $\chi$ for which particles stick to each other. For $\chi \leqslant \chi_{c}^{(2)}$ particles deform along the path $0 \rightarrow \delta_{\max }^{p} \rightarrow \delta_{\max } \rightarrow \delta_{0} \rightarrow \delta_{\min } \rightarrow \delta_{\mathrm{c}}$ and then keep oscillating with $k_{2}$ stiffness, $\delta_{\mathrm{c}}$ being one of the extrema of the oscillation, see Fig. 3.9c. From Eq. (3.24e), applying conservation of energy along $\delta_{\min } \rightarrow \delta_{\mathrm{c}}$, we get

$$
\frac{1}{2} m_{r} v_{f}^{2}-\frac{1}{2} m_{r} v_{0}^{2}=-\frac{1}{2} k_{c} \delta_{\min } \delta_{0}+\frac{1}{2} k_{c} \delta_{\mathrm{c}}^{2},
$$

with $v_{f}=0$, and re-writing in terms of $k_{c}$ and $\delta_{\max }^{p}$ leads to

$$
\frac{\delta_{\mathrm{c}}^{(2)}}{\delta_{\max }^{p}}=\sqrt{\left[\frac{\left(k_{p}-k_{1}\right)^{2}}{k_{p}\left(k_{p}+k_{c}\right)}-\frac{k_{1}^{2}}{k_{c} k_{p}}+\frac{k_{1}}{k_{c}}\right]-\frac{m_{r}}{k_{c}} \frac{v_{i}^{2}}{\left(\delta_{\max }^{p}\right)^{2}}} .
$$


In terms of the dimensionless parameters, this yields

$$
\frac{\delta_{\mathrm{c}}^{(2)}}{\delta_{\max }^{p}}=\sqrt{\frac{\eta^{2}}{(1+\eta)(1+\beta+\eta)}+\frac{\eta}{\beta(1+\eta)}-\frac{\chi^{2}}{\beta}}=\frac{\chi}{\sqrt{\beta}}\left|e_{n}^{(2)}\right|
$$

where $\left|e_{n}^{(2)}\right|$ denotes the absolute value of the result from Eq. (3.29).

In Fig. 3.10, the evolution of $\delta_{c} / \delta_{\max }^{p}$ with $\chi$ is reported, showing perfect agreement of the analytical expressions in Eqs. (3.34) and (3.38), with the numerical solution of a paircollision. In the sticking regime, the stopping overlap increases with $\chi$, and reaches a maximum at $\chi=1$,

$$
\delta_{\mathrm{c}}^{\max } / \delta_{\max }^{p}=\sqrt{\frac{\beta \eta^{2}-\eta-\beta-1}{\beta(1+\eta)(1+\eta+\beta)}}
$$

which depends on the the adhesivity $\beta$ and the plasticity $\eta$ only. For $\chi>1$, dissipation gets weaker, relatively to the increasing initial kinetic energy, and $\delta_{\mathrm{c}}^{(2)} / \delta_{\max }^{p}$ decreases until it reaches 0 for $\chi=\chi_{c}^{(2)}$.

\subsubsection{Dependence on Adhesivity $\beta$}

In the previous subsections, we studied the dependence of the coefficient of restitution $e_{n}$ on the scaled initial velocity $\chi$ for fixed adhesivity $\beta$, whereas here the dependence of $e_{n}$ on $\beta$ is analyzed.

A special adhesivity $\beta^{*}$ can be calculated such that $e_{n}=0$ for $\chi=1$, which is the case of maximum dissipation and leads to sticking only at exactly $\chi=1$. From Eq. (3.28), we get

$$
1+\beta^{*}+\eta-\beta^{*} \eta^{2}=0
$$

so that

$$
\beta^{*}=\frac{1}{\eta-1} .
$$

In Fig. 3.11, we plot the coefficient of restitution as function of the scaled initial velocity $\chi$ for different values of adhesivity $\beta$. For $\beta<\beta^{*}$, in Fig. 3.11, the coefficient of restitution $e_{n}$ decreases with increasing $\chi<1$, reaches its positive minimum at $\chi=1$, and increases for $\chi>1$. In this range, the particles (after collision) always have a non-zero relative separation velocity $v_{f}$. When $\beta=\beta^{*}, e_{n}$ follows a similar trend, becomes zero at $\chi=1$, and increases with increasing scaled initial velocity for $\chi>1$. This is the minimum value of adhesivity for which $e_{n}$ can become zero and particles start to stick to each other. For $\beta=\beta^{*}$, the two critical values coincide, $\chi_{c}^{(1)}=\chi_{c}^{(2)}=1$. If $\beta>\beta^{*}, e_{n}$ decreases and becomes zero at $\chi=\chi_{c}^{(1)}<1$, it remains zero until $\chi=\chi_{c}^{(2)}>1$, and from there increases with increasing initial velocity. Hence, we can conclude that the sticking of particles and then range of velocity for which this happens, is affected by material properties of both particles. Indeed Zhou et al. [229] presented similar conclusion about deposition efficiency in cold spray. 


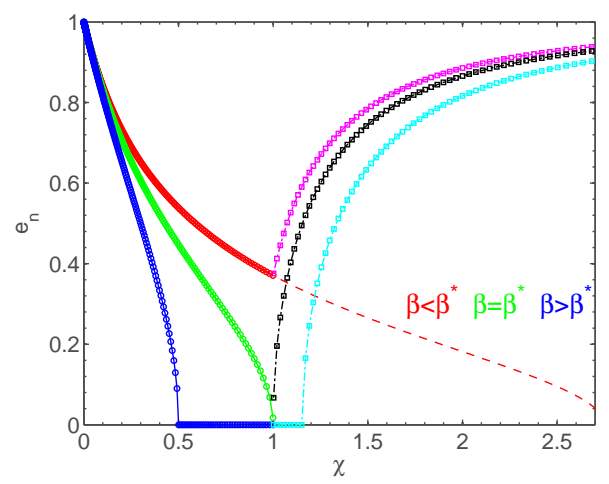

Figure 3.11: Coefficient of restitution $e_{n}$ plotted against the scaled initial velocity $\chi$. Circles with different colors correspond to different adhesivity $\beta$ (red for $\beta<\beta^{*}$, green for $\beta=\beta^{*}$ and blue for $\beta>\beta^{*}$ ) for $\chi \leq 1$, while magenta, black and cyan squares correspond to the respective values of $\beta$ for $\chi>1$. Other parameters used are $k_{1}=10^{2}, k_{p}=5 \times 10^{2}$, and different $k_{c}$ (all in units of $\mathrm{Nm}^{-1}$ ), i.e. $\eta=4$ and $\beta / \beta^{*}=1 / 3,1$, and 3 , with $\beta^{*}=1 / 3$. The dashed red line represents the solution with the tuned fully plastic model with a new $\phi_{f}{ }^{\prime}=0.5$ and newly calculated $k_{p}{ }^{\prime}$, see Appendix 3.C.

\subsection{Conclusions}

Various classes of contact models for non-linear elastic, adhesive and elasto-plastic particles, are reviewed in this chapter. Instead of considering the well understood models for perfect spheres of homogeneous (visco) elastic or elasto-plastic materials, here we focus on a special class of mesoscopic adhesive elasto-plastic models, aimed to describe the overall behavior of assemblies of realistic (different from perfectly homogeneous spheres) fine particles.

The contact model by Luding [102] is extended and generalized by adding short-ranged (non-contact) interactions. The model is critically discussed and compared to alternative approaches which are classified in Sub-Section 3.1.2. The influence of the model parameters on the overall impact behavior is discussed, focusing on the irreversible, adhesive, elastoplastic part of the model and combining all the elements (non-contact, hysteretic, contact and viscous dissipation) at the end. The model is simple yet it catches the important features of particle interactions that affect the bulk behavior of a granular assembly, like elasticity, plasticity and contact adhesion. It is mesoscopic in spirit, i.e. it does not resolve all the details of every single contact, but it is designed to represent the ensemble of many contacts in a bulk system. The goal is to propose a rich, flexible and multi-purpose granular matter model, which is realistic and allows to involve large numbers of particles.

When the dependence of the coefficient of restitution, $e$, on the relative velocity between particles is analyzed, two sticking regimes, $e=0$, show up. These are related to different 
sources of dissipation in the system.

(i) As previously reported in the literature (see e.g. Refs. [23, 36, 74, 186, 197]) the particles stick to each other at very low impact velocity. This can happen due to irreversible shortrange non-contact interaction. The threshold velocity, below which the particles stick, is directly related to the magnitude of the non-contact adhesive force $f_{a}$. (ii) With increasing velocity, $e$ increases and then decreases until the second sticking regime is reached. This is a result of the plastic/adhesive dissipation mechanisms in the hysteretic contact model.

At small impact velocity, the details of the contact model are of minor importance. At higher velocities, for a sufficiently low value of jump-in force $f_{a}$ the contribution of the (irreversible) non-contact forces can be neglected. In the limit of weak $f_{a}$ and for moderate-high velocities, the contact component of the coefficient of restitution $e_{n}$ is examined analytically using simple energy conservation arguments.

The results are derived in a closed analytical form, by phrasing the behavior in terms of dimensionless parameters (plasticity, adhesivity and initial velocity) and the range of impact velocities of the second sticking regime is predicted.

For still increasing relative velocity, beyond the sticking region, $e_{n}$ starts increasing again. This regime involves a change of the physical behavior of the system and resembles for example material with an elastic core like asphalt (stone with bitumen layer). Completely plastic behavior can be reproduced by the same model without any change, just by tuning two input parameters $k_{p} \hat{\mathrm{A}}$ and $\phi_{f}$, such that low velocity collision dynamics is kept unaffected but the maximal plastic overlap is reached only at much higher impact velocities. This modification provides the high velocity sticking regime for high velocity, as expected for plastic material. The existence of a high velocity rebound, as predicted by our model, has been observed experimentally and numerically in cold spray [168, 189, 228, 229, 232] and can be expected for elastic core with a thin plastic shell. As shown in appendix 3.C, the model is flexible enough to model the "unusual" behavior (which might be relevant to other applications described above), as well as the well-known decrease of the coefficient of restitution with impact velocity.

In the sticking regime, due to the lack of dissipation on the unloading / re-loading branch the sticking particles oscillate around their equilibrium position. However, the real collision between two-particles is dissipative in nature. Since viscosity hinders analytical solutions, a few simulation results with viscosity are presented. With viscosity, the unloading/re-loading is not reversible elastic anymore. Thus with time the particles undergo a damped oscillation and approach a static contact with finite overlap.

In the last part of the chapter a section is dedicated to the asymptotic behavior of $e_{n}$ at high impact velocities. We observe that the asymptotic behavior is directly related to the choice of the interaction law and its details. For low adhesivity, the coefficient of restitution is found 
to decay as $e_{n} \sim v_{i}{ }^{-1 / 2}$, which is due to the empirical choice of the unloading stiffness in the model. Further analysis on this feature is possible in the future, when new data from modern experimental techniques involving fine powders or core-shell materials becomes available for numerical calibration and validation [86].

The application of the present extended model to many-particle systems (bulk behavior) is the final long-term goal, see Ref. [107] as an example, where the non-contact forces were disregarded. An interesting question that remains unanswered concerns a suitable analogy to the coefficient of restitution (as defined for pair collisions) relevant in the case of bulk systems, where particles can be permanently in contact with each other over long periods of time, and where impacts are not the dominant mode of interaction.

The interest of widely different communities viz. granular physics, particle technology, interstellar dust, asphalt or cold-spray in the dependence of restitution coefficient/deposition efficiency on impact velocity is considerable. We hope our study helps to connect these widely different communities by providing an overview and in particular a flexible multipurpose contact model valid in many practically relevant situations.

\section{A Appendix}

In this appendix, we present in detail the effect of viscous damping, different interpolations. We also present asymptotic soultions with different interpolations. Finally we also show the unloading and re-loading behavior of mesoscopic particles. Section 3.A.1 is devoted to show the effect of viscous damping on the coefficient of restitution. Section 3.A.2 is devoted to the asymptotic behavior of the coefficient of restitution as function of the impact velocity. In section 3.A.3, we discuss the effect of a linear and square-root interpolations on the coefficient of restitution as a function of impact velocity. In section 3.B, we show the time evolution of the kinetic and potential energies during the collision. In section 3.C, we present a method to tune the slope of plastic branch $k_{p}{ }^{\prime}$ for any given $\phi_{f}{ }^{\prime}$, such that the collision dynamics for lower plastic deformation $\delta_{0}<\delta^{p}{ }_{0}$ is intact. Finally in section 3.D, we present the unloading and re-loading behavior of an agglomerate, i.e. its effective, mesoscopic force-displacement relation, which clearly is different from the contact force law applied at the primary particle contacts.

\section{A.1 Effect of Viscosity}

Since real physical systems also can have additional dissipation modes that are, e.g., viscous in nature, in this section we study the behavior of the collision when viscosity is present $\left(\gamma_{0}>0\right)$ and compare it with the non-viscous case $\left(\gamma_{0}=0\right)$. Note that any non-linear viscous damping force can be added to the contact laws introduced previously, however, for the sake of simplicity we restrict ourselves to the simplest linear viscous law as given as second term 
in Eq. (3.3). However it is important to choose the correct viscous damping term for the force law to get the correct behavior as explained in [89, 100, 108, 220]. In Fig. 3.12, we plot the contact force against the overlap, and the overlap against time, during collisions for a constant value of $\chi=1$ and different $\beta$, for $\gamma_{0}=5 \times 10^{-3}$.

When $\beta<\beta^{*}$, see Fig. 3.12a and Fig. 3.12b, the contact ends when the adhesive force $-k_{c} \delta$ goes back to zero, for both cases, with and without viscosity. This is since the viscosity is relatively small and does not contribute enough to the total dissipation to make the particles stick.

For the critical adhesivity $\beta=\beta^{*}$, reported in Fig. 3.12c, without viscosity the overlap between the particles still goes down to exactly zero at the end of the collision, with all kinetic energy dissipated. For $\gamma_{0}>0$, dissipation brings this marginal collision case into the sticking regime and the particles stay in contact at $\delta>0$. This can be seen clearly in Fig. 3.12d, where the particles undergo a damped oscillatory motion with amplitude depending on the residual velocity $v_{f}$ (the amplitude is very small due to small residual velocity).

For larger values $\beta>\beta^{*}$, the overlap does not reach 0 , neither for $\gamma_{0}=0$ nor for $\gamma_{0}>0$, see Fig. 3.12e. In both cases, the particles stick and remain in contact with a finite overlap. Without viscosity, the particles keep oscillating along the slope $k_{2}$, while in the case with viscosity the oscillation is damped and kinetic energy vanishes. During loading and unloading the apparent slope changes with time due to the additional viscous force that leads to the dissipation of energy. Waiting long enough, for some oscillation cycles, the particles stick to each other with a finite overlap and zero relative kinetic energy. The difference is displayed in Fig. 3.12f, where for $\gamma_{0}=0$ the particles keep oscillating with constant amplitude, whereas, for $\gamma_{0}>0$, the particles undergo a damped oscillatory motion, until the velocity becomes 0 at $\delta>0$. The time evolution of the overlap in Fig. 3.12f resembles that of the displacement evolution in Ref. [62], where the authors studied sticking of particles in Saturn's rings. ${ }^{2}$

\section{A.2 Asymptotic Solutions}

In this subsection, we focus on the case $\chi \leq 1$, and study the asymptotic behavior of the coefficient of restitution as function of the impact velocity.

For the sake of simplicity, let us start with an elasto-plastic system without adhesion, i.e.

2. In general, one could add a viscous law that is proportional to $k_{2}-k_{1}$ or to a power of overlap $\delta$, such that the jump-in viscous force in (e) at the beginning of the contact is not there, however, we do not go into this detail. 


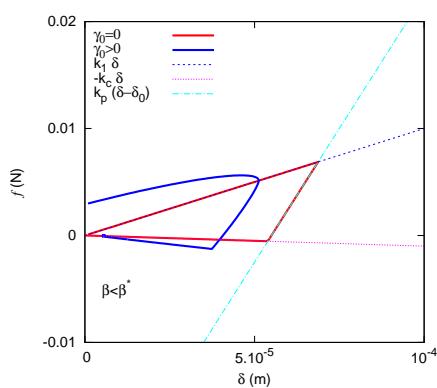

(a)

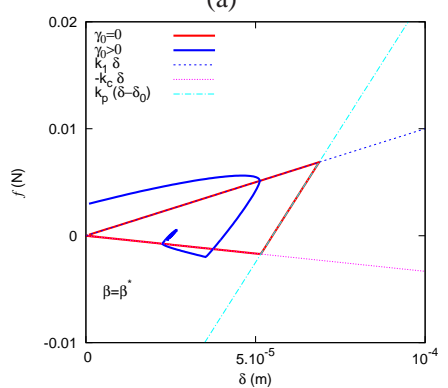

(c)

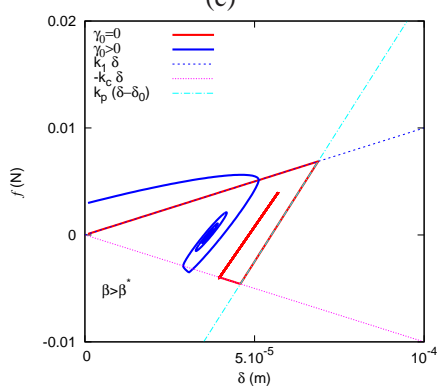

(e)

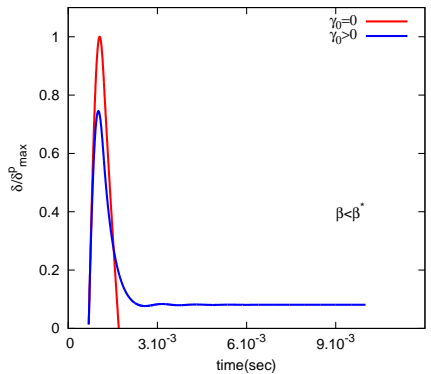

(b)

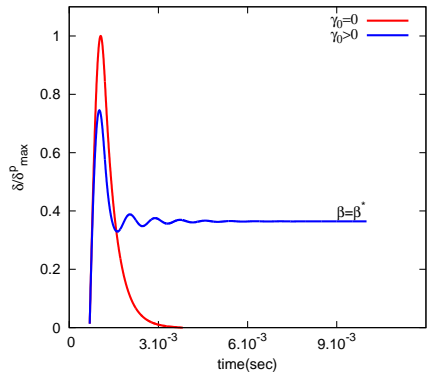

(d)

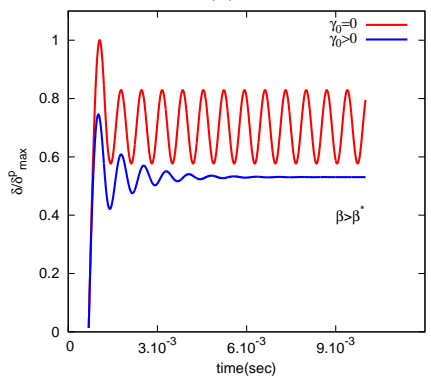

(f)

Figure 3.12: (a), (c), (e) Contact forces plotted against overlap and (b), (d), (f) time evolution of $\delta / \delta_{\max }^{p}$ for pair collisions with parameters $k_{1}=10^{2}, k_{p}=5 \times 10^{2}$ and different $k_{c}=10$, 33.33, and 100, (units $\mathrm{Nm}^{-1}$ ), i.e. with $\eta=4, \beta<\beta^{*}, \beta=\beta^{*}$ and $\beta>\beta^{*}$, for the same situations as shown in Fig. 3.11. The red and blue lines represent the data in the presence and absence of viscosity respectively, where $\gamma_{0}=5 \times 10^{-3}$, (unit $\mathrm{Nm}^{-1} \mathrm{sec}$ ). 
$k_{c}=0$, in Eq. (3.28) such that

$$
e_{n}^{(1)}(\eta, \beta=0, \chi<1)=\sqrt{\frac{1}{1+\eta \chi}},
$$

inserting the definitions of $\eta, \beta$ and $v_{p}$,

$$
e_{n}^{(1)}\left(\beta=0, v<v_{p}\right)=\sqrt{\frac{1}{1+\frac{k_{p}-k_{1}}{k_{1}} \frac{v_{i}}{\sqrt{\frac{2 k_{1}}{m} \delta_{\max }^{p}}}}},
$$

using Eq. (3.13), where we defined $S=\frac{k_{p}-k_{1}}{k_{1} \delta_{\max }^{p}}$ and assuming $\omega_{o}=\sqrt{\frac{2 k_{1}}{m}}$, we get

$$
e_{n}^{(1)}\left(\beta=0, v<v_{p}\right)=\sqrt{\frac{1}{1+\frac{S v_{i}}{\omega_{0}}}} .
$$

Eq. (3.41c) is exactly the same as Eq. (5) in [222]. For non-cohesive particles, and in the range $v<v_{p}$ we get exactly the same solution as Walton and Braun [222].

Further to study the asymptotic solution

$$
e_{n}^{(1)}(\eta, \beta=0, \chi<1)=\sqrt{\frac{1}{1+\eta \chi}} \approx(\eta \chi)^{-1 / 2}
$$

with the approximation valid for $\eta \chi \gg 1$. Since the scaled velocity is moderate, $\chi<1$, the condition requires a large plasticity, i.e., a strong difference between the limit stiffness and the plastic loading stiffness, $\eta \gg 1$ (or $k_{p} \gg k_{1}$ ). In Fig. 3.13, we plot the coefficient of restitution against the scaled initial velocity $\chi$ for three different values of $\eta=k_{p} / k_{1}$, together with the power law prediction of Eq. (3.42). We observe, that for the smallest $\eta$ (red circle and line), the approximation is far from the data, while for higher $\eta$, the approximation works well even for rather small velocities $\chi \approx 0.1$.

Next, when studying the elasto-plastic adhesive contact model, $\beta>0$ and $\beta \ll 1$, again, we restrict ourselves to values of $\eta$ such that asymptotic condition $\eta \chi \gg 1$ is satisfied. Hence, Eq. (3.28) can be approximated as

$$
e_{n}^{(1)}(\eta, \beta, \chi<1) \approx \sqrt{\frac{1}{\eta \chi}-\beta},
$$

as long as $\eta \chi \gg \beta \geq 0$ and $\frac{1}{\eta}>\beta$ holds.

In Fig. 3.14, we plot the coefficient of restitution against the scaled initial velocity $\chi$ for different values of $\beta$ and superimpose the approximation, Eq. (3.43). For small $\beta$ and large $\chi$, 


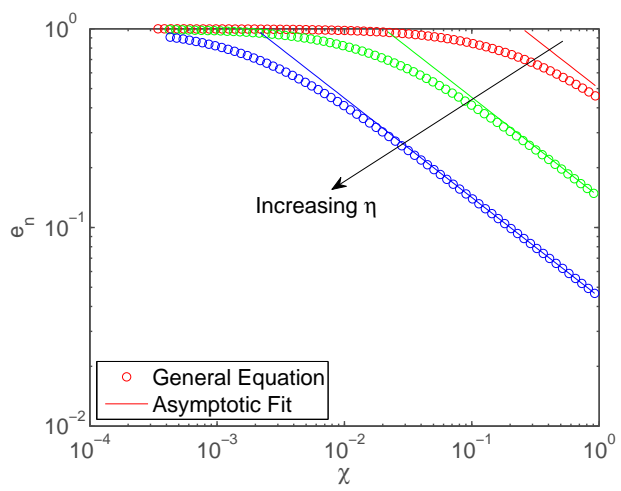

Figure 3.13: The coefficient of restitution is plotted against the scaled initial velocity $\chi$ in $\log \log$-scale for $\beta=0$ and three values of $\eta=5,50$, and 500, with the other parameters as in Fig. 3.7. Red, green and blue circles denote, respectively, the solution of Eq. (3.42), while the solid lines represent the approximation for high scaled impact velocity and large plasticity $\eta \gg 1$.

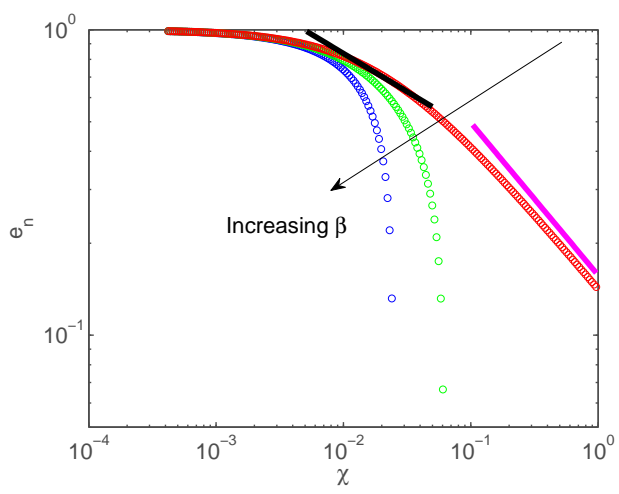

Figure 3.14: Log-log plot of the coefficient of restitution against the scaled initial velocity $\chi$ for four different values of $\beta=0.01,0.1$, and 1.0, with $\eta=50$. Red, green and blue circles denote the respective solutions of the general equation, Eq. (3.28), solid black line represents power law $e_{n} \sim v^{-1 / 4}$, while magenta line denotes $e_{n} \sim v^{-1 / 2}$. 
one observes good agreement between the full solution and the approximation. Differently, for the highest values of $\beta$ the approximation is not valid. Due to the adhesive force, for large $\chi$, with increasing $\beta$, the deviation from the $\chi^{-1 / 2}$ power law becomes increasingly stronger, leading to the sticking regime, as discussed in the previous subsections. On the other hand, for smaller velocities, one observes a considerably smaller power-law, resembling the well-known $\chi^{-1 / 4}$ power law for plastic contacts, as indicated by the dashed line in Fig. 3.14.

\section{A.3 Dependence on interpolation}

The choice of the interpolation rule for the unloading stiffness $k_{2}$ in Eq. (3.27) is empirical. Therefore, for $\delta_{\max } / \delta_{\max }^{p}<1$, a different choice could be:

$$
k_{2}\left(\delta_{\max }\right)=k_{1}(1+\eta \sqrt{\chi}) .
$$

Inserting Eq. (3.44) into Eq. (3.23) leads to a different expression for the normal coefficient of restitution $e_{n}^{(1)}$, which for high values of $\eta \sqrt{\chi}$, and for small $\beta$, reduces to

$$
e_{n} \propto \sqrt{\eta}(\chi)^{-1 / 4}
$$

A similar power law prediction for moderate velocities has been previously obtained by Thornton et al. in Ref. [197], using a non-linear Hertzian loading and unloading. Fig. 3.15 shows the agreement between the power law approximation $\chi^{-1 / 4}$ and Eq. (3.23) with the alternative interpolation rule (3.44), for moderate velocities. The choice of different interpolation laws for $k_{2}$ shows the flexibility of the model and requires input from experiments to become more realistic. The convexity of linear interpolation for zero cohesion is very similar to that of low $\beta$ in Fig. 3.11.

\section{B Energy Picture}

This appendix shows the energies of two particles during contact, where the difference between the different branches of the contact model, namely irreversible/unstable or reversible/elastic, will be highlighted.

In Fig. 3.16, the time-evolution of kinetic and potential energy is shown; the graphs can be viewed in parallel to Figs. 3.9a and 3.9b. In Fig. 3.16a, we plot the kinetic and potential energy of the particles against time for low initial velocity $\chi<\chi_{c}^{(1)}$, corresponding to Fig. $3.9 \mathrm{a}$, for which dissipation is so weak that particles do not stick. The kinetic energy decreases from its initial value and is converted to potential energy (the conversion is complete at $\delta_{\max }$ ). Thereafter, the potential energy drops due to the change between the loading and unloading slope from $k_{1}$ to $k_{2}$. The potential energy decreases to zero (at the force-free overlap $\delta_{0}$ ), where it is converted to (less) kinetic energy. Then the kinetic energy decreases further due 


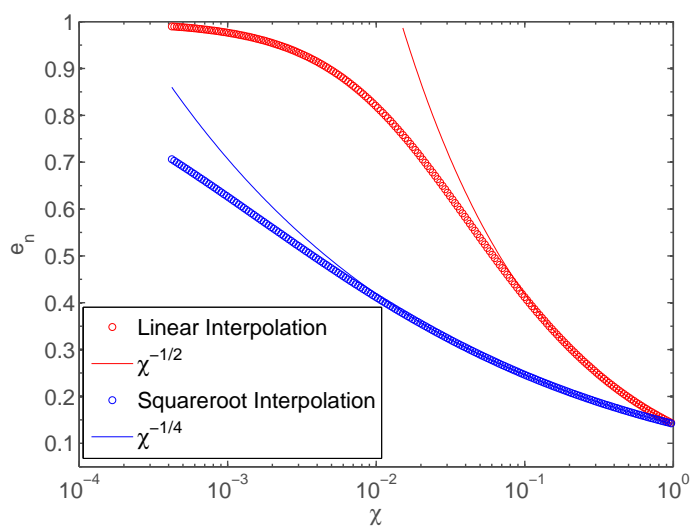

Figure 3.15: Semi-log plot of the coefficient of restitution as function of the scaled initial velocity $\chi$, using different interpolation rules for $k_{2}$, for pair collisions with $\eta=50$ and $\beta=0$. The symbols denote the solutions of the general equation, Eq. (3.42) with linear interpolation (red circles) or square root interpolation (blue circles), as given in Eq. (3.44). The red and blue solid lines represent the approximations for high impact velocity $e_{n} \sim \chi^{-1 / 2}$ and $e_{n} \sim \chi^{-1 / 4}$.

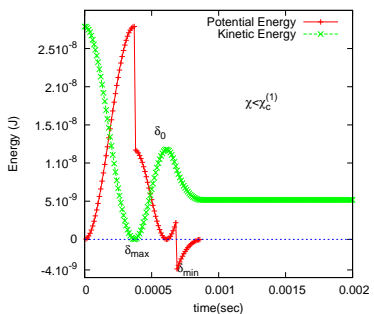

(a)

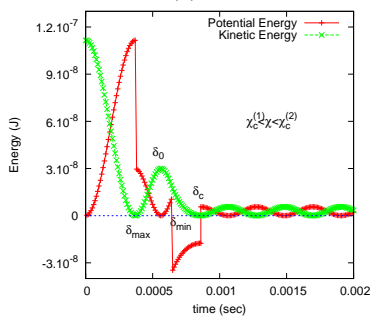

(c)

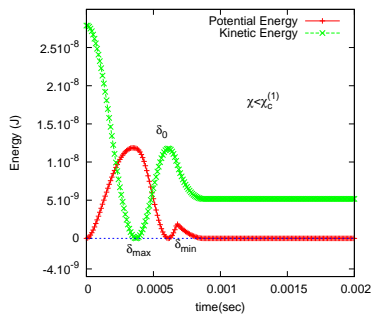

(b)

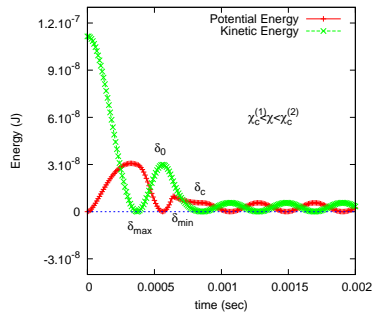

(d)

Figure 3.16: (a,c) Kinetic and (irreversible, plastic, "potential") energy of the particles, and $(b, d)$ kinetic and available (elastic) potential energy (for re-loading) of the particles, plotted against time for pair collisions with $k_{1}=10^{2} \mathrm{Nm}^{-1} \cdot k_{p}=5 \times 10^{2} \mathrm{Nm}^{-1}$, and $k_{c}=10^{2} \mathrm{Nm}^{-1}$, i.e. $\eta=4$ and $\beta=1$. The initial velocity $\chi$ is $\chi=0.34(\mathrm{a}, \mathrm{b})$ and $\chi=0.69(\mathrm{c}, \mathrm{d})$, in the regimes defined in the inset of each plot. 
to the acting adhesive force. At $\delta_{\min }$ the increasing potential energy drops to a negative value due to the change in unloading slope from $k_{2}$ to the adhesive (instable) slope $-k_{c}$. From there it increases from this minimum, negative value to zero, for $\delta=0$. From here the kinetic energy remains constant and the potential energy stays at zero, since the particles are separated.

In Fig. 3.16b, we plot the time evolution of kinetic and potential energy that the particles would have if un-/re-loading would take place at that moment, along the branch with slope $k_{2}$, namely the available (elastic) potential energy. This energy increases from zero at $t=0$, and reaches a maximum when the kinetic energy becomes zero (note that it is not equal to the initial kinetic energy due to the plastic change of slope of $k_{2}$.) Thereafter, the available potential energy decreases to zero at the force-free overlap $\delta_{0}$. For further unloading, the available potential energy first increases and then drops rapidly on the unstable branch with slope $-k_{c}$. The change in sign of the unloading slope, from $k_{2}$ to $-k_{c}$, is reflected in the kink in the curve at $\delta_{\min }$. Note, that comparing Figs. 3.16a and 3.16b, the available potential energy always stays positive, while the total, plastic "potential" energy drops to negative values after the kink at $\delta_{\min }$.

Figs. 3.16c and 3.16d show the time evolution of kinetic and potential energy (total and available, respectively) for an initial velocity $\chi_{c}^{(1)}<\chi<\chi_{c}^{(2)}$ in the sticking regime, see Fig. 3.9b. In Fig. 3.16c, a similar trend as that of Fig. 3.16a is observed until the potential energy becomes negative at $\delta_{\min }$. The difference to the case of smaller impact velocity is that at this point, the kinetic energy is less than the magnitude of the negative potential energy and hence first reaches zero, i.e., the particles stick. At this point, the (plastic) potential energy increases and jumps to a positive value indicating the change in sign of the unloading slope from $-k_{c}$ to $k_{2}$. Finally, it oscillates between this positive value at $\delta_{\mathrm{c}}$, exchanging energy with the kinetic degree of freedom. When the available potential energy is plotted in Fig. 3.16d, a similar trend as that of Fig. $3.16 \mathrm{~b}$ is observed up to the kink at $\delta_{\min }$. Here, the two energies have comparable values when they reach $\delta_{\min }$ and the kinetic energy decreases to zero with a non-zero available potential energy, which causes the contact to re- and un-load along $k_{2}$.

\section{C Tuning of parameters to increase the plastic range}

We assume that the reference dimensionless plasticity depth be $\phi_{f}$, which is e.g. calculated based on the maximal volume fraction related arguments of a multi-particle assembly, and $k_{p}$ be the reference limit stiffness. We propose a new $\phi_{f}{ }^{\prime}>\phi_{f}$ which represents the new dimensionless plasticity depth (our choice or calculated based on another volume fraction) and a new value of $k_{p}{ }^{\prime}$ such that the tuned model resembles the reference for $\delta_{0}<a_{12} \phi_{f}$ and becomes plastic for $a_{12} \phi_{f}<\delta_{0}<a_{12} \phi_{f}^{\prime}$. At $\delta_{0}=a_{12} \phi_{f}$, with $a_{12}$ being reduced radius, Eq. 


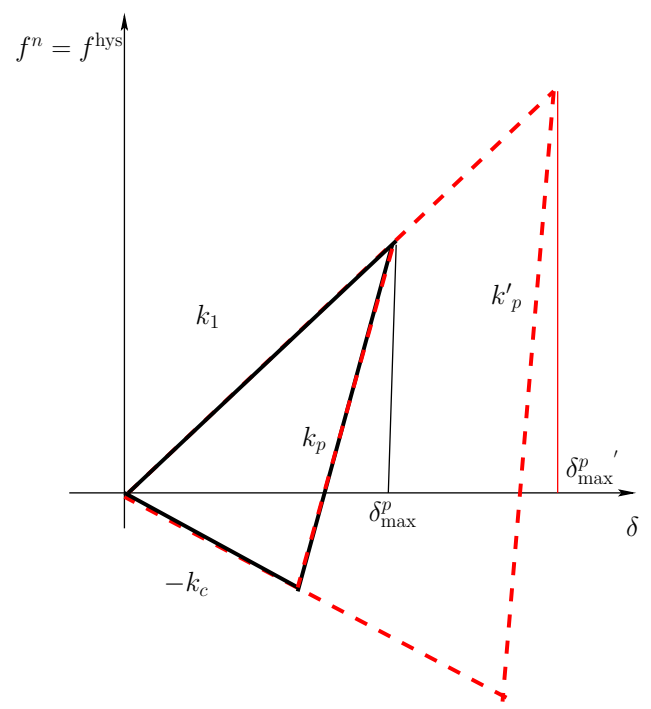

Figure 3.17: Force-displacement law for elasto-plastic, adhesive contacts superimposed on the irreversible contact force law. The black solid line represents the force law for reference input parameters $\phi_{f}$ and $k_{p}$, while the dashed red line represents the same for a new chosen $\phi_{f}^{\prime}$ and newly calculated $k_{p}{ }^{\prime}$ resembling a wider plastic regime of the particle deformation.

(3.12) reads

$$
k_{p}=k_{2}=k_{1}+\left(k_{p}^{\prime}-k_{1}\right) \delta_{\max }^{p} / \delta_{\max }^{p},
$$

since all parameters except $\phi_{f}$ and $k_{p}$ remain unchanged. Using this definition in Eq. (3.12) we arrive at

$$
\frac{\left(k_{p}-k_{1}\right)^{2}}{k_{p} \phi_{f}}=\frac{\left(k_{p}{ }^{\prime}-k_{1}\right)^{2}}{k_{p}{ }^{\prime} \phi_{f}^{\prime}}
$$

which gives the new limit stiffness

$$
k_{p}{ }^{\prime}=\frac{2 k_{1}+A B+\sqrt{(A B)^{2}+4 k_{1} A B}}{2},
$$

where $A=\frac{\left(k_{p}-k_{1}\right)^{2}}{k_{p}}$ and $B=\frac{\phi_{f}{ }^{\prime}}{\phi_{f}}$.

Using Eq. (3.48), we can calculate values of the new limit plastic stiffness $k_{p}{ }^{\prime}$ for any given $\phi_{f}{ }^{\prime}$, such that the collision dynamics for lower plastic deformation $\delta_{0}<\delta^{p}$ is intact, while the range of plastic deformation is enhanced, depending on the chosen $\phi_{f}{ }^{\prime}>\phi_{f}$. 


\section{D Agglomerate compression and tension test}

Goal of this appendix is to show the unloading and re-loading behavior of an agglomerate, i.e. its effective, mesoscopic force-displacement relation, which clearly is different from the contact force law applied at the primary particle contacts. We will report incomplete detachment and partly/weaker elastic response for re-loading after various different compressive and tensile loading amplitudes.

The system considered here is an agglomerate (cubic) of size $L_{0}=0.115$, made of $N=1728$ primary particles of diameter $d_{0}=0.01$ (with some variation in size to avoid monodisperse artefacts), just like in Ref. [102]. The cubic sample was first compressed (pressure-sintered) with a dimensionless wall stress $d_{0} p_{s} / k_{p}=0.02$ to form a stable, rather dense agglomerate or "tablet". The stress is first released to a value $2 \cdot 10^{-5}$, i.e. $p_{r} / p_{s}=10^{-3}$ for all walls. Then various uni-axial, unconfined tension/compression tests are carried out applying either further tension or compression starting from the released state of the sample [102]. The simulation parameters are same as in Ref. [102] (table 2), except for the cohesion that is set here to a rather small intensity, $k_{c} / k_{p}=0.2$, rolling and sliding friction coefficients that are double as large, $\mu_{r}=\mu_{o}=0.2$, and viscous damping of those degrees of freedom, $\gamma_{r} / \gamma=$ $\gamma_{o} / \gamma=0.1$, which also is larger than that of the reference situation.

The force-displacement curves for the tests at different amplitudes are shown in Figs. 3.18 and 3.20 for tension and compression tests respectively. All simulations in Figs. 3.18 and 3.20 start from the same configuration, i.e. the released state mentioned above and is indicated by the black circle at point $(0,0)$. These plots represent the mesoscopic contact model of agglomerates consisting of multiple primary particles and their geometrical surface configurations and change in shape during the tests.

Fig. 3.18 shows the force-displacement curve for an unconfined uniaxial tension test. The black arrow shows the unloading/tension path, and finally arrows with different colors show the re-loading paths for different deformation amplitudes, as given in the inset. Each of the tests, when it reaches the original strain at zero, is then repeated for three more cycles. Note that repeated cyclic loading remains on the same branch with positive slope, displaying the elastic nature of the contact, while it is not completely, perfectly detached. The contact surface is changing plastically by restructuring of the primary particles and surely is not flat, see Fig. 3.19, as one would expect for ideal, homogeneous, plastic materials. For the largest amplitude, the behavior is not perfectly elastic anymore, since the first plastic effects show up. For deformations as large as 0.2 of the primary particle diameter, $d_{0}$, before re-loading (arrow with positive slope on the red curve) has mostly, but not completely lost mechanical contact. The complete detachment of the assembly happens for much higher amplitude, than what is expected from a 2 particle interaction. Note that the contact model of the primary particles is behaving elasto-plastically $\left(\phi_{f}=0.05\right)$ on the scale of only $0.05 d_{0}$; the reversible, elastic un-/re-loading is thus not due to the primary particle contact model, since it stretches 


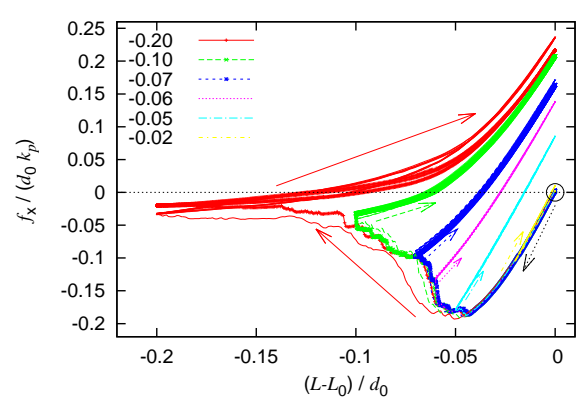

Figure 3.18: Dimensionless force-displacement curve for an unconfined uni-axial tension test (negative horizontal axis), with the various different deformation amplitudes $D_{x}$ given in the inset. The downward arrow indicates the direction of first tensile unloading, while the upwards-right arrows indicate the change of force during re-loading. Except for the red curve, all these branches are reversible, for repeated un-/re-loading.

to four times $\phi_{f} d_{0}$ and even higher displacements. Finally, in order to confirm that this is not an effect of viscosity, qualitatively, the thick lines are simulations performed four times slower than those with thin lines.

In Fig. 3.19, a few snapshots during the tensile deformation are presented. The first snapshot corresponds to the undisturbed sample, while the others are increasing tensile deformation amplitudes. Note that these deformations are much larger than in Fig. 3.18. The contact is completely lost only at the extreme, final deformation in Fig. 19(g). In Fig. 3.19, it is also visible that the contact surface has developed a roughness of the size of several primary particles; the first visible gap is opened at a total deformation of $D_{x} \sim d_{0}$, and the contact is lost only at $D_{x} \sim 8 d_{0}$, when the last of the thin threads breaks. The elastic, irreversible tension branch, however, is strongly developed only for much smaller $D_{x} \sim d_{0} / 5$.

Complementing the tension test above, Fig. 3.20 shows the behavior of the same sample during compression cycles. The values given in the inset indicate the amplitude of un-/reloading. The smallest amplitudes remain elastic throughout, while plastic deformation kicks in for $D_{x}>0.1$ (see the red curve). However, the unloading and re-loading take place on the same branch, i.e. a new elastic branch (e.g. for $D_{x}=0.2$ ). For even larger amplitudes, e.g. the yellow curve with $D_{x}=0.3$, the continuous damage/plastic destruction of the sample (by considerable irreversible re-arrangement during each cycle). Again, thick lines indicate simulations four times slower, which shows a small quantitative difference, but qualitative agreement even for the largest amplitude/rate. The snapshots in Fig. 3.21 show the continuous plastic deformation of the sample at large strains. 


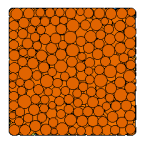

(a)

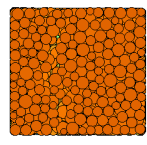

(b)

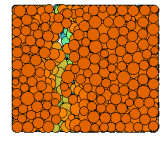

(c)

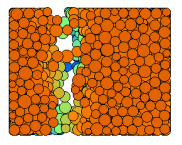

(d)

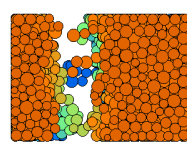

(e)

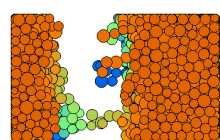

(f)

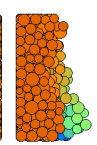

(g)

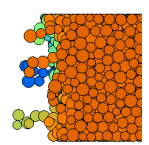

Figure 3.19: Snapshots of the tablet-sample during (large) tensile deformations for $D_{x}=(L-$ $\left.L_{0}\right) / d_{0}=0$ (a), 0.81 (b), 1.8 (c), 3.1 (d), 4.7 (e), 7.4 (f), and $8.6(\mathrm{~g})$. The primary particles are colored according to their distance from the viewer (red, green, blue is increasing distance).

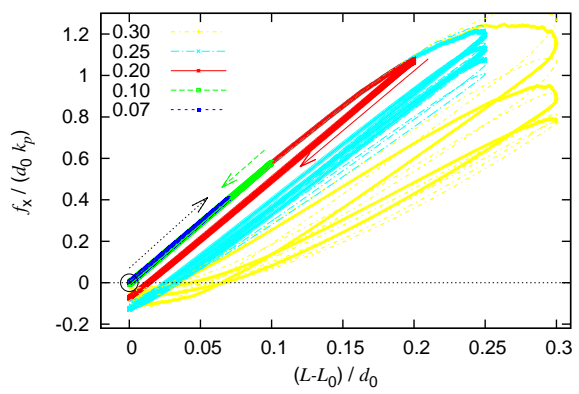

Figure 3.20: Dimensionless force-displacement curve for the same sample as in Fig. 3.18, but under compressive initial loading and un-/re-loading. The values in the inset indicate the maximal amplitudes $D_{x}$. 


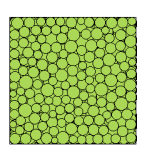

(a)

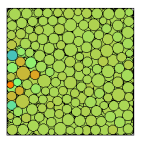

(b)

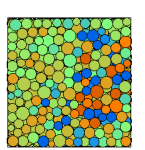

(c)

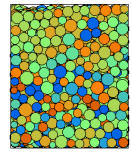

(d)

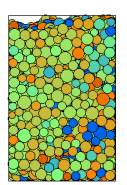

(e)

Figure 3.21: Snapshots of the tablet sample during compression at (large) deformations $D_{x}=\left(L-L_{0}\right) / d_{0}=0(\mathrm{a}), 0.01$ (b), 0.3 (c), 0.8 (d), and 1.8 (e). The color code is small stress (green) and compressive/tensile larger stress (red/blue) averaged/isotropically per primary particle. 


\title{
CHAPTER 4
}

\section{Effect of friction and cohesion on behavior of granular materials*}

\begin{abstract}
The effect of particle friction and cohesion on the steady state shear strength and the contact anisotropy of a granular material is studied using Discrete Element Simulations (DEM). For non-cohesive frictional material, when shear stress and fabric anisotropy are analyzed locally, as functions of pressure and shear rate, they are found to increase with contact friction; and saturation is reached for high friction with the strong contact network dominating. From a microscopic point of view, we analyze the probability distribution functions (PDFs) of both normal and tangential forces. They behave in a similar fashion, i.e., the probability of weak forces and heterogeneity are increasing with friction.

For cohesive powders, the relation between shear stress and confining pressure becomes non-linear. Interestingly the contact number density stays almost unaffected, while the structure anisotropy of the contact network decreases with increasing cohesion, hinting at a redistribution within the contact network without affecting the total number of contacts.
\end{abstract}

*. Based on A. Singh, V. Magnanimo, and S. Luding. Effect of friction and cohesion on anisotropy in quasistatic granular materials under shear. AIP Conference Proceedings, 1542(1):682-685, 2013 


\subsection{Introduction}

What do sand, rice, coffee powders and cocoa powder have in common? They all are granular materials: a collection of non-Brownian, macroscopic particles with dissipative interactions. Their intrinsic nature leads to great deal of interesting phenomena like segregation, jamming, clustering, arching and shear-band formation [72]. It is now well established that the microstructure of granular matter plays a significant role in the overall constitutive behavior. Finding a connection between the continuum behavior and kinematics at microscale is a great challenge and involves the so-called micro-macro transition [11, 102-105, 215]. On this respect, particle simulations are relatively recent powerful tools that allow to track individual particles with complex interaction by solving Newton's laws of motion. The micro-macro transition is often applied on small, seemingly homogeneous representative volume elements (RVE)s. An alternative is to use an inhomogeneous geometry where static and dynamic, flowing zones i.e. high density and dilated zones co-exist - at various confining pressure levels. In these kind of systems, by local averaging over adequate representative volume elements (RVE)s: inside which all particles are assumed to behave similarly, one can obtain local continuum relations covering a wide range of system states. Such a procedure has been performed systematically in two-dimensional Couette ring shear cell [91, 92], and three dimensional split-bottom ring shear cell [103-106]. One special property of this set-up is the fact that a wide, stable shear band initiates at the bottom split and remains far away from side walls. The free surface of the split-bottom shear cell allows to scan a range of confining pressure, due to weight of the material, and is determined by the filling height.

Under shear, the microstructure of a grain packing develops anisotropic features in both stress and contact network. The anisotropy depends on preparation history of the material, and at the same time on the contact and particle properties such as stiffness, roughness and angularity of particles $[5-8,190,195]$. In this chapter, we study the effect of particle contact properties ( friction and cohesion) on the steady state macroscopic properties of the system. Further we analyze the problem at the microlevel and study the effect of particle friction on the steady state behavior, by investigating the probability distribution (PDFs) of forces along the eigen-directions of the local strain rate tensors.

The chapter is organized in 4 main parts. Section 4.2 describes the model system specifying the geometry, particle properties and interaction laws. In section 4.3, the results from samples with different inter-particles friction on the force distribution are presented. Finally, 4.4 is the section dedicated to discussion of results.

\subsection{Model System Geometry}

In this section, the methodology of our molecular dynamics types simulations is briefly discussed. The details about particle properties are briefly presented in Sec. 4.2.1 and we show 
our numerical setup in Sec. 4.2.2. In Sec. 4.2.3, we briefly discuss our averaging methodology.

\subsubsection{Numerical simulations}

Discrete Element Methods (DEM), provide numerical solutions of Newton's equations of motion based on the specification of particle properties viz. stiffness, density, radius and an interaction law like Hertzian/Hookean [1, 33]. Simulation methodology and material parameters used in this study are the same as in our previous work [105]. The normal contact force is related to overlap through a linear contact law, $f_{n}=k_{n} \delta_{n}$, with stiffness $k_{n}$, if $\delta \geq 0$. In tangential direction, the force $f_{t}=k_{t} \delta_{t}$ is also proportional to the tangential displacement of the contact point, with a stiffness $k_{t}$. The tangential force is limited by Coulombs law for sliding $f_{t} \leq \mu_{p} f_{n}$, i.e. for $\mu_{p}=0$ tangential forces do not exist. For more details on the contact model see [105] and references therein.

The system is filled with $N \approx 37000$ spherical particles with density $\rho=2000 \mathrm{~kg} / \mathrm{m}^{3}=$ $2 \mathrm{~g} / \mathrm{cm}^{3}$. The average size of particles is $a_{0}=1.1 \mathrm{~mm}$, with a homogeneous size-distribution of the width $1-\mathcal{A}=1-\langle a\rangle^{2} /\left\langle a^{2}\right\rangle=0.18922$ (with $a_{\min } / a_{\max }=1 / 2$ ). The stiffness constant of the particles is $k=100 \mathrm{Nm}^{-2}$. The rolling and torsion friction are inactive. The normal and tangential viscosities are $\gamma_{n}=0.002 \mathrm{~kg} \mathrm{~s}^{-1}$ and $\gamma_{t} / \gamma_{n}=1 / 4$. In order to study the influence of contact friction, we analyzed the system for the following set of friction coefficients:

$\mu_{\mathrm{p}} \in[0.0,0.005,0.01,0.02,0.05,0.1,0.2,0.5,1.0]$

To study the effect of contact cohesion, an adhesive elasto-plastic contact model [102] is used to simulate cohesive particles, involving an elastic limit stiffness $k_{p}=500 \mathrm{Nm}^{-1}$, a plastic stiffness $k_{1}=100 \mathrm{Nm}^{-1}$, and an adhesive "stiffness" $k_{c}$. The simulations were run for different values of the non-dimensional cohesive strength $\beta=k_{c} / k_{1}=[0,0.1,1]$, in order to focus on effect of cohesion the particle friction is set artificially small to $\mu_{\mathrm{p}}=0.01$.

\subsubsection{Simulation Setup}

We perform numerical simulation in the same setup described earlier in Sec. 2.1.1.1. It consists of two concentric cylinders, with inner and outer cylinder radii as $R_{i}=0.0147 \mathrm{~m}$ and $R_{O}=0.11 \mathrm{~m}$ respectively, with a split radius being $R_{S}=0.085 \mathrm{~m}$ (as shown in Fig. 2.2). The concentric cylinders rotate relative to each other around the symmetry axis (the dotdashed line). The ring shaped split at the bottom separates the moving and static parts of the system, where a part of the bottom and the outer cylinder rotate at the same rate. The system is filled with $N \approx 3.7 \times 10^{4}$ spherical particles with density $\rho=2000 \mathrm{~kg} / \mathrm{m}^{3}=2 \mathrm{~g} / \mathrm{cm}^{3}$ up to height $H$. The cylindrical walls and the bottom are roughened due to some (about $3 \%$ of the total number) attached/glued particles, as explained in [103-105]. When there is a relative 
motion at the split, a shear band propagates from split position $R_{S}$ upwards and inwards and remains far away from cylinder-walls and bottom in most cases. Since we are interested in the quasi-static regime, the rotation rate of outer cylinder is chosen to be $\frac{\Omega}{2 \pi}=0.01 \mathrm{~s}^{-1}$, such that the inertial number $I \ll 1[118]$.

\subsubsection{Averaging and micro-macro procedure}

The averaging procedure used in this study as same as discussed briefly in Sec. 2.3.2.1. From the simulations, we calculate stress and fabric tensors, which are represented as $\sigma_{i j}$ and $F_{i j}$ respectively. It is important to mention that for the rotation rate $\frac{\Omega}{2 \pi}=0.01 \mathrm{~s}^{-1}$, the contribution of the kinetic stress to the total shear stress $\sigma$ is much smaller compared to the contact stress. Hence, we ignore the dynamical stress and concentrate on the contact stress.

For both stress and fabric tensors, we can calculate the eigenvalues and define the volumetric part $T_{\mathrm{v}}=\left(T_{1}+T_{2}+T_{3}\right) / 3$ (pressure $p$ and $F_{\mathrm{V}}$ for stress and fabric respectively) and deviatoric component as $T_{\mathrm{dev}}=\sqrt{\left(\left(T_{1}-T_{2}\right)^{2}+\left(T_{2}-T_{3}\right)^{2}+\left(T_{3}-T_{1}\right)^{2}\right) / 6}\left(\sigma_{\mathrm{dev}}\right.$ and $F_{\mathrm{dev}}$ for stress and fabric respectively). The pressure is the isotropic stress, while $\sigma_{\mathrm{dev}}$ quantifies the normal stress difference. The volumetric fabric $F_{\mathrm{V}}$ represents the contact number density, while the deviatoric fabric $F_{\mathrm{dev}}$ quantifies anisotropy of the contact network.

\subsection{Results}

For a given confining stress (pressure) and preparation history, the material can only resist shear up to a certain deviatoric (shear) stress, called the "yield stress", beyond which it fails $[90,170,171]$. When yield points $\left(p^{(y)}, \sigma^{(y)}\right.$ dev $)$ are collected in the $\sigma_{\mathrm{dev}}-p$-plane, a yield locus can be identified, that fully describes the failure behavior of the material, i.e. its transition from static to dynamic state. In addition, when the material is sheared continuously for a long time, it reaches a steady state which is characterized by a steady state yield stress, i.e. the stress needed to keep the material in motion, $\left(p^{(c)}, \sigma_{\operatorname{dev}}^{(c)}\right)$, also referred to as the critical state yield or "termination" locus. For simple non-cohesive granular materials, the termination locus can be predicted from a Coulomb type criterion as a straight line with a slope that can be called the (critical) steady state macroscopic friction coefficient $s_{D}:=$ $\left(\sigma^{(c)} \operatorname{dev}\right) / p^{(c)}$. When cohesion is introduced at the contact, a more complicated picture appears as described in Ref. [107].

When the material fails, shear strain gets localized in regions called as shearband that, in case of the split-bottom shear cell, is stable, rather wide with error-function shape, but nevertheless stays away from the walls in our system. In order to identify the established steady state shearbands, Luding in $[103,105]$ found that for $f_{0}=0.01$, only part of the system with local shear rate above a threshold $\dot{\gamma}^{*}=0.08$ can be assumed to be in the critical state. Hence for this study we only consider data with local shear rate above a threshold $\dot{\gamma}^{*}$ 
In the following sections, we study the effect of particle friction coefficient on the macroscopic behavior of the material by studying its effect on stress and fabric tensors. At the same time, we also study how particle friction affects the microscopic properties of material such as the probability density functions (PDFs) $P\left(f^{*}\right)$ in the steady state. Later the effect of contact cohesion on the steady state macroscopic behavior is also briefly discussed.

\subsubsection{Effect of particle friction on macroscopic behavior}

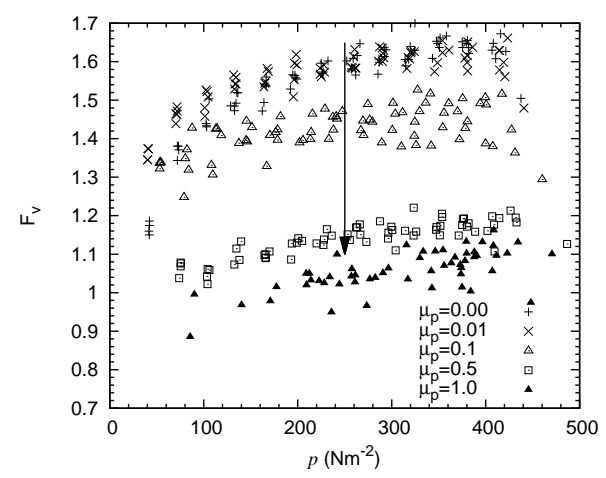

Figure 4.1: Volumetric fabric $F_{\mathrm{v}}$ plotted against pressure $p$. The different symbols corresponds to data from simulations with different particle friction coefficients, as given in the inset.

In Fig. 4.1, we plot the volumetric fabric against pressure. For a given $\mu_{\mathrm{p}}, F_{\mathrm{v}}$ shows a slight increase with pressure ( small drop is observed at the highest pressure level). Corresponding to a value of pressure, different points correspond to different values of local strain rate in the system. With increasing $\mu_{p}, F_{\mathrm{V}}$ decreases. Enhanced friction at contact increases the stability of the systematic and reduces the number of contacts required to achieve a stable configuration, leading to reduction in the contact number density.

Fig. 4.2 shows the variation of deviatoric fabric $F_{\mathrm{dev}}$ against pressure $p$. Opposite to the volumetric fabric, the fabric anisotropy increases with friction. That can be related to the decrease in $F_{\mathrm{v}}$ : As the packing becomes looser anisotropy becomes stronger. Upon shearing the probability of particle contacts to establish in favorable directions could be higher due to presence of empty voids in systems with larger $\mu_{\mathrm{p}}$.

In Fig. 4.3, we plot the shear stress ratio $s_{D}$ against the contact friction coefficient $\mu_{\mathrm{p}}$. We observe that for $\mu_{\mathrm{p}}=0, s_{D}$ is non-zero due to interlocking between the particles [144]. $s_{D}$ increases rapidly and reaches an asymptote at high $\mu_{\mathrm{p}}$. 


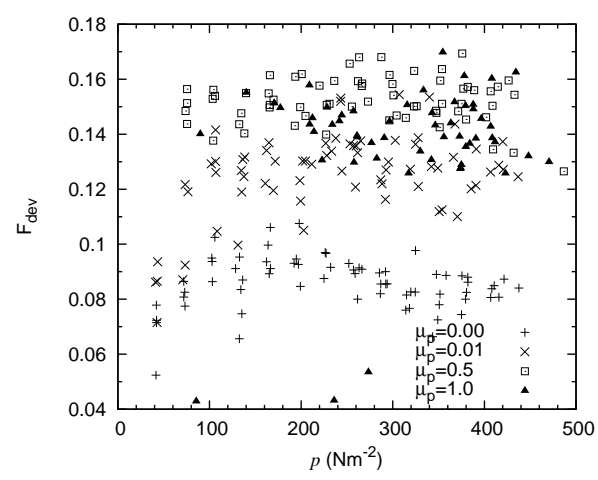

Figure 4.2: Deviatoric fabric $F_{\text {dev }}$ plotted against pressure $p$. The different symbols corresponds to simulations using different particle friction coefficients, as given in the inset.

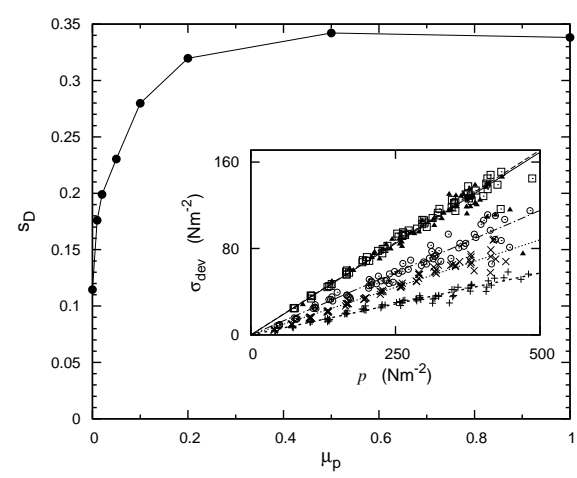

Figure 4.3: Shear stress ratio $s_{D}$ plotted as function of particle friction coefficient. The inset shows deviatoric (shear) stress $\sigma_{\text {dev }}$ plotted against pressure $p$. The different symbols correspond to simulations using different particle friction coefficients $+\left(\mu_{\mathrm{p}}=0\right), \mathrm{x}\left(\mu_{\mathrm{p}}=\right.$ $0.01), \mathrm{o}\left(\mu_{\mathrm{p}}=0.05\right), \square\left(\mu_{\mathrm{p}}=0.5\right)$ and $\mathbf{\Delta}\left(\mu_{\mathrm{p}}=1.0\right)$.

4.3.1.0.1 Contribution of strong and weak contacts Many studies such as $[3,4,154$, $188,195]$, have suggested that the contact network can be partitioned into two complementary subnetworks: a strong subnetwork that accounts for the whole deviatoric stress, and a weak subnetwork that contributes only to the mean stress. Moreover, the strong subnetwork develops higher structural anisotropy compared to the weak subnetwork, which remains almost isotropic. In this section we re-examine our data from previous section in terms of strong and weak force- transmission networks and contribution each makes to both stress and fabric tensors for different values of particle friction $\mu_{\mathrm{p}}$.

Fig. 4.4 illustrates the relative contributions of subnetworks with $f^{*}<1$ and $f^{*}>1$ to the 
deviatoric stress $\sigma_{\mathrm{dev}}$ and deviatoric fabric $F_{\mathrm{dev}}$, together with the overall network. Fig. 4.4a clearly show that contribution of the weak subnetwork to $\sigma_{\text {dev }}$ is negligible. In Fig. $4.4 \mathrm{~b}$ we plot the contribution of weak and strong subnetworks to $F_{\mathrm{dev}}$. In agreement with previous studies [154, 195], a much stronger fabric anisotropy characterizes the strong subnetwork, while the weak subnetwork is mostly isotropic. It is noteworthy that $F_{\mathrm{dev}}$ for both subnetworks is positive because of the definition of deviator, but we expect the main eigenvectors of strong and weak subnetworks to have orthogonal directions. As an initial attempt, for a given particle friction, we assume the deviatoric fabric to be independent of pressure and depend only on $\mu_{\mathrm{p}}$, i.e., $F_{\mathrm{dev}}\left(\mu_{\mathrm{p}, \mathrm{p}}\right)=F_{\mathrm{dev}}\left(\mu_{\mathrm{p}}\right)$.

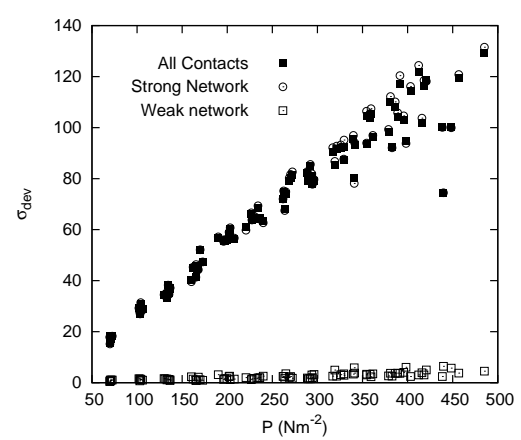

(a)

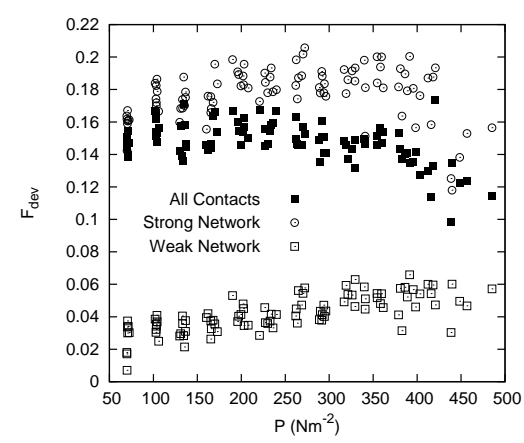

(b)

Figure 4.4: Contribution of weak, and strong subnetworks to (a) $\sigma_{\operatorname{dev}}$ and (b) $F_{\operatorname{dev}}$ plotted against pressure $P$.

Fig. 4.5a displays the normalized shear stress $s_{D}=\sigma_{\operatorname{dev}} / P$ as a function of $\mu_{\mathrm{p}}$ for the two subnetworks and the overall system. We observe that for the whole range of particle friction $\mu_{\mathrm{p}}$, the strong subnetwork carries almost all $s_{D}$. System with $\mu_{\mathrm{p}}=0$ has finite $s_{D}$ due to interlocking of contacts. Whole network and the strong subnetwork show similar variation in $s_{D}$ as a function of $\mu_{\mathrm{p}}$, i.e. an initial increase and then saturation for high $\mu_{\mathrm{p}}$.

Fig. $4.5 \mathrm{~b}$, displays $F_{\mathrm{dev}}$ as a function of $\mu_{\mathrm{p}}$ for the strong and weak subnetworks and the complete network. We observe that the strong subnetwork carries higher anisotropy than the overall network. It is interesting to note that, similarly $F_{\mathrm{dev}}$ also starts with a non-zero value for zero $\mu_{\mathrm{p}}$ i.e. the contact network for frictionless particles is also anisotropic. It increases with initial increase in $\mu_{\mathrm{p}}$ until it saturates for high $\mu_{\mathrm{p}} \geq 0.3$.

\subsubsection{Effect of particle friction on force distribution}

To better understand the macroscopic observations in the previous section, we focus on its micromechanical origin by looking at the probability distribution function of forces. Micro- 


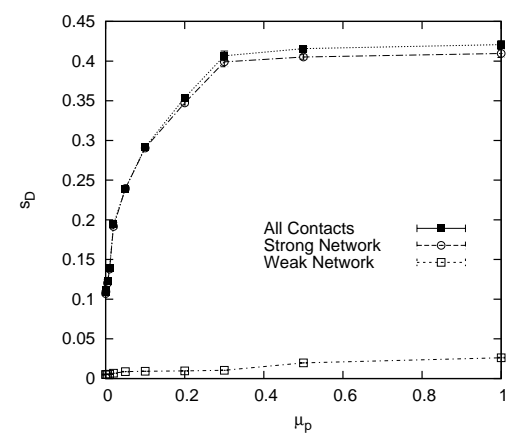

(a)

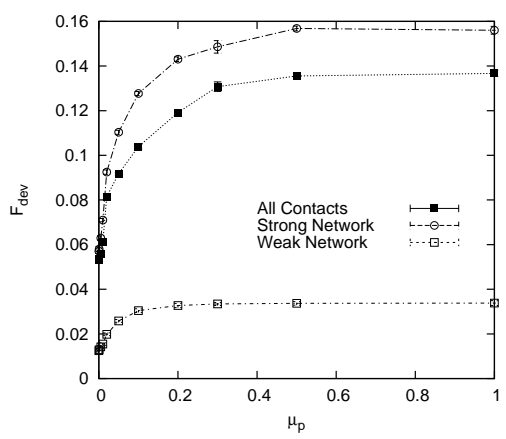

(b)

Figure 4.5: Contribution of weak, and strong subnetworks to (a) $s_{D}=\sigma_{\mathrm{dev}} / P$ and (b) $F_{\mathrm{dev}}$ plotted against contact coefficient of friction $\mu_{\mathrm{p}}$.

scopically, the nature of internal force network is of great importance in understanding various macroscopic observations. One important quantitative way to study this is to measure $P(f)$ of the normal forces $f$ between neighboring particles, which carries the information about the inhomogeneities in the force network.

Pioneering work of Liu et al. [97] using carbon paper technique indicated that the number of contacts carrying a given force decreased as the magnitude of the force increased. From the work of $[8,18,27,49,124,133,174]$, it was shown that the probability distribution is also sensitive to preparation history, packing disorder and various particle properties like contact friction.

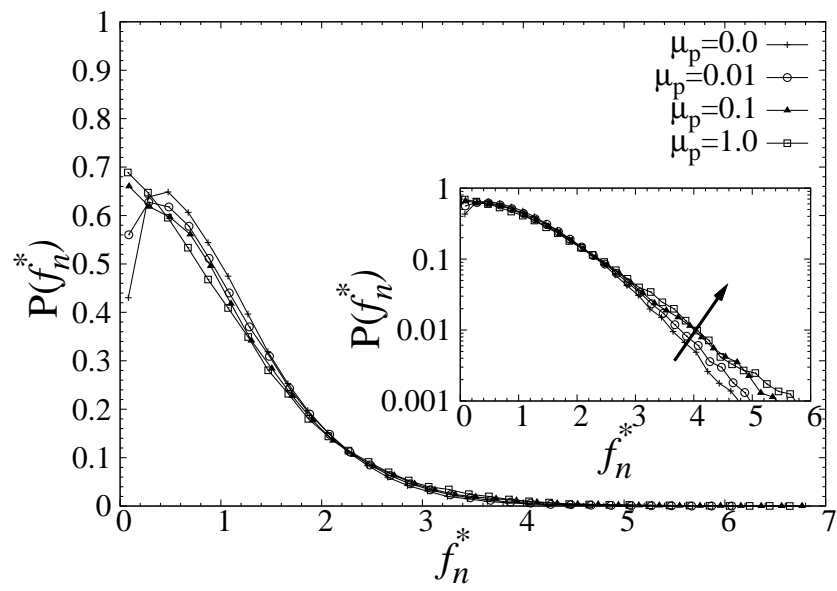

Figure 4.6: Probability density functions of normalized normal forces for the data inside the shear band. The inset shows the same on a log-linear scale. 
4.3.2.0.2 Normal forces The probability distributions for normalized normal forces $f^{*}=$ $f /\langle f\rangle$ are shown in Fig. 4.6 for different values of $\mu_{\mathrm{p}}$ (for the sake of clarity we show only 4 values of $\left.\mu_{\mathrm{p}}\right)$. We observe a very similar behavior for $P\left(f^{*}\right)$ in the range of strong forces $f^{*}>1$, a decrease in $P\left(f^{*}\right)$ with increasing $f^{*}$. However, the contact friction affects the distribution of weak forces $f^{*}<1$, as for $\mu_{\mathrm{p}}=0.0$ and 0.01 , the distribution bends down as $f^{*} \rightarrow 0$, but does not tend to zero, while an opposite is observed for high $\mu_{\mathrm{p}}$. The difference in shape of $P\left(f^{*}\right)$ for $f^{*}<1$ was also observed by Silbert et al. [174] while comparing threedimensional packings, and Azéma et al. [8, 9] for three-dimensional packings of spheres and polyhedra. This shows that the fraction of contacts carrying weak forces increases with increasing $\mu_{\mathrm{p}}$, even though the total number of contacts decrease. The inset of the figure also shows that with increasing friction, the tail of distribution in Fig. 4.6 gets longer (from $\mu_{\mathrm{p}}=$ 0.0 to 1.0 ), stating that the inhomogeneity in the contact network increases with friction. Moreover, the tail of the distribution getting wider with friction reflects the effect on force chains, that get stronger with increasing $\mu_{\mathrm{p}}$.

Furthermore, we look at $P\left(f^{*}\right)$ along the principal eigen-directions of the local strain rate tensor of the overall network [46, 47]. Fig. 4.7 displays $P\left(f^{*}\right)$ of forces for contacts aligned with the compressive directions of local strain rate tensor normalized by the mean force of overall network. We observe that the tail of the distribution gets longer, with increasing $\mu_{\mathrm{p}}$, i.e. the increase in particle friction leads to inhomogeneity in the forces along the compressive direction. For all range of particle friction, tensile subnetwork always stays more homogeneous than the compressive subnetwork, and as shown in Fig. 4.8 no clear trend in the tail of the distribution is observed with increasing $\mu_{\mathrm{p}}$. This implies that friction mainly affects the force distribution along the compressive direction.

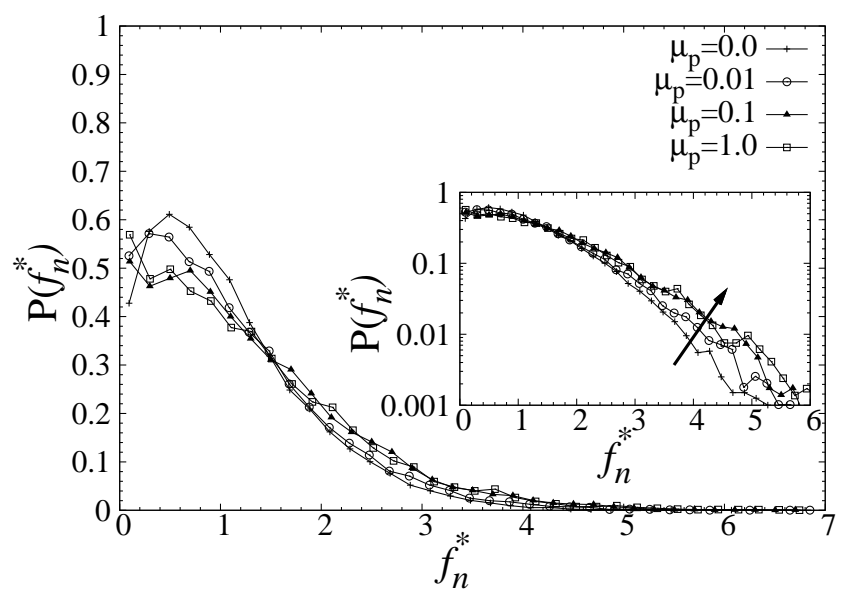

Figure 4.7: Probability density functions of normal forces along the compressive direction normalized by overall normal forces for the data inside the shear band. The inset shows the same on a log-linear scale. 


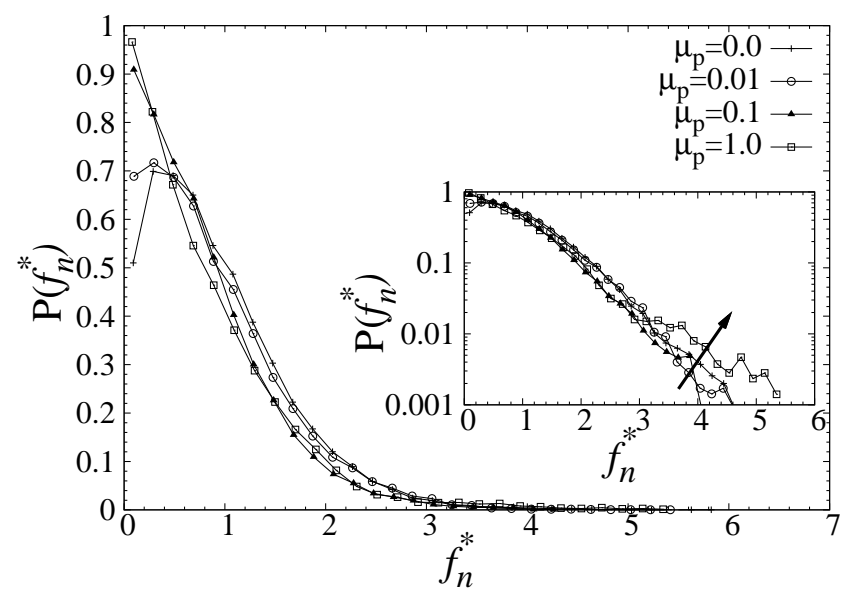

Figure 4.8: Probability density functions of normal forces along the tensile direction normalized by overall normal forces for the data inside the shear band. The inset shows the same on a log-linear scale.

4.3.2.0.3 Tangential forces Fig. 4.9 shows the distribution of the tangential forces, that behaves very similar to $P\left(f^{*}\right)$ for normal forces, with a friction dependent cup for $f^{*}<1$.

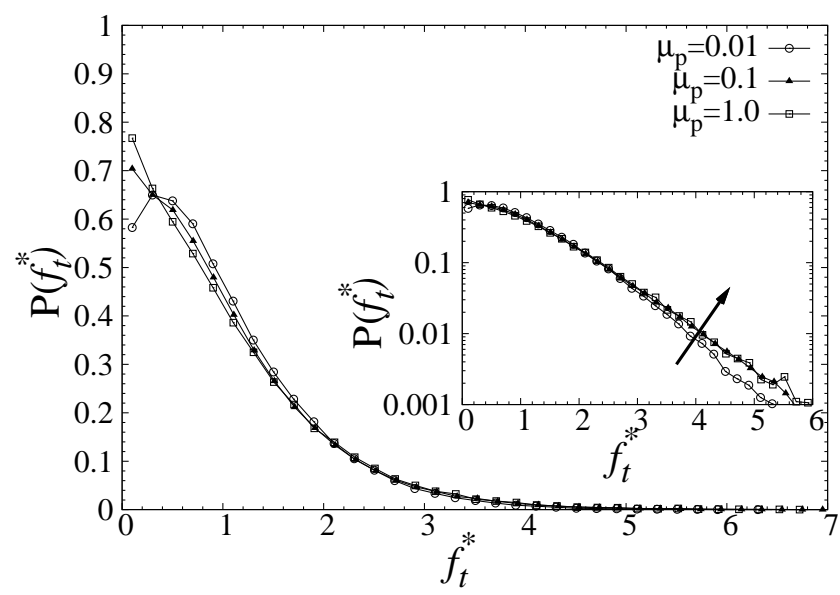

Figure 4.9: Probability density functions of normalized tangential forces for the data inside the shear band. The inset shows the same on a log-linear scale.

For completeness, we also analyze the probability distribution of tangential forces along the compressive and tensile directions, as done previously. Surprisingly, the behavior is now different between the normal and tangential components, as the latter does not show any trend with friction in the compressive direction (Fig. 4.10). This implies that friction does not affect the tangential forces along the eigenvectors, but only the whole distribution of 
forces, displaying a different mechanism between normal and tangential forces.

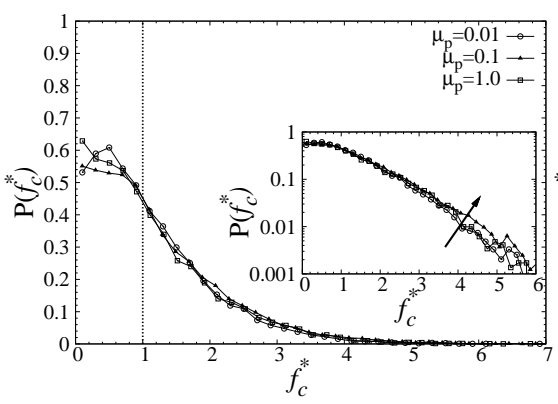

(a)

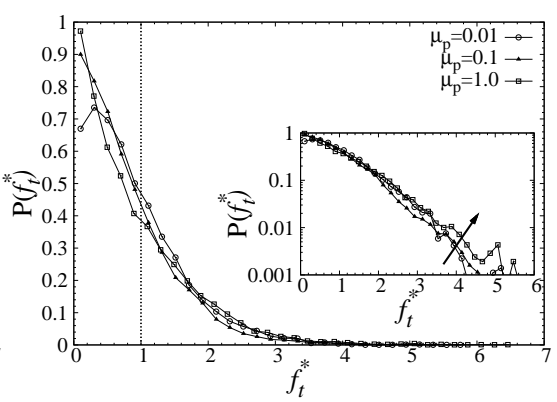

(b)

Figure 4.10: Probability density functions of normalized tangential forces along (Left) compressive and (Right) tangential directions, for the data inside the shear band. The inset shows the same on a log-linear scale.

\subsubsection{Anisotropy of force networks}

Fig. 4.11 shows the $P\left(f^{*}\right)$ for normal forces along the eigen-directions for different values of $\mu_{\mathrm{p}} . P\left(f^{*}\right)$ of overall network lies in between the $P\left(f^{*}\right)$ of forces in compressive and tensile directions. For all values of $\mu_{\mathrm{p}}$, we observe that the $P\left(f^{*}\right)$ of weak forces $\left(f^{*}<1\right)$ along the tensile direction is higher compared to that in the compressive direction, which is intuitive as the majority of contacts will have small forces in the tensile direction. However, as the value of force increases, $P\left(f^{*}>1\right)$, the probability along the compressive directions overcomes the one in tensile direction. We also observe that, with increasing $\mu_{\mathrm{p}}$, the difference between the distributions along compressive and tensile directions increases, meaning that the anisotropy in force network increases.

Fig. 4.12 displays the same comparison for tangential forces. Again, no trend of $P\left(f_{t}^{*}\right)$ can be inferred from the figure, as a confirmation that friction does not affect the behavior of the tangential components along the eigenvectors.

\subsubsection{Effect of contact cohesion on macroscopic behavior}

In Fig. 4.13, we plot the (shear) deviatoric stress $\sigma_{\mathrm{dev}}$ against pressure $p$, for different cohesive-parameters $\beta$. With increasing $\beta$, the relation between shear stress $\sigma_{\operatorname{dev}}$ and pressure becomes non-linear, as studied in more detail in Ref. [107]. Here, we focus on the effect of particle cohesion on the volumetric and deviatoric fabric. The data collapse when $F_{\mathrm{v}}$ is plotted against $p$ for different $\beta$, as shown in Fig. 4.15.

In Fig. 4.14, we plot $F_{\mathrm{dev}}$ against pressure $p$. The non-cohesive case $(\beta=0)$ is identical to 


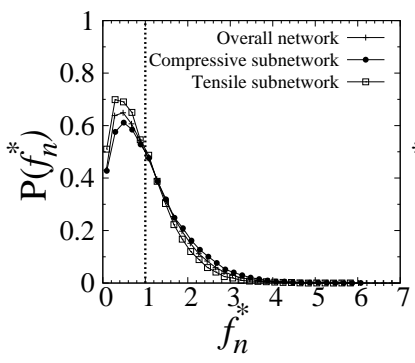

(a)

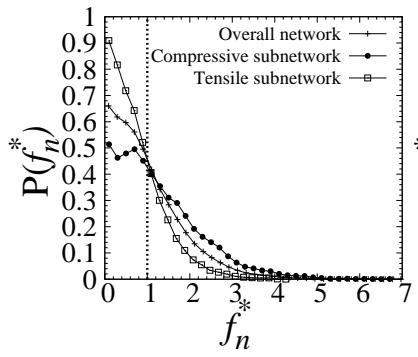

(c)

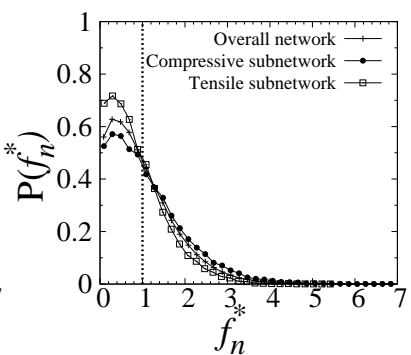

(b)

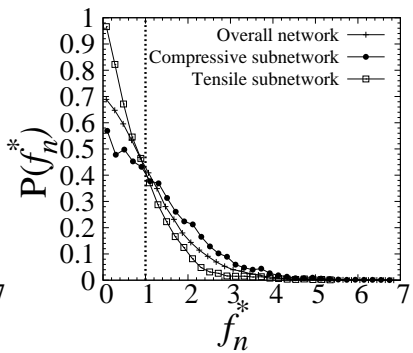

(d)

Figure 4.11: Probability density functions of normal forces along the compressive, tensile directions, and overall network for $\mu_{\mathrm{p}}=$ (a) 0 , (b) 0.01 , (c) 0.1 , and (d) 1.0 in linear scale for the data inside the shear band. The forces are normalized by the mean normal force of the overall network.

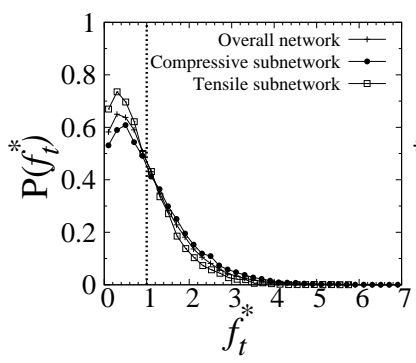

(a)

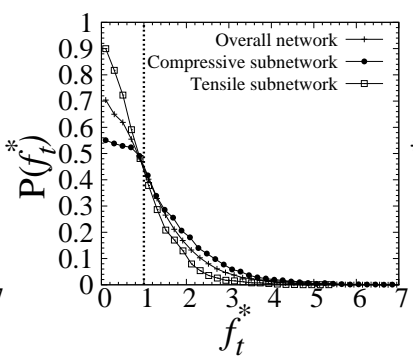

(b)

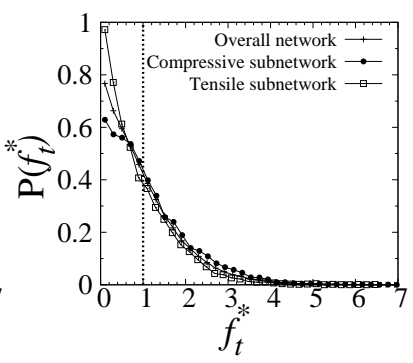

(c)

Figure 4.12: Probability density functions of tangential forces along the compressive, tensile directions, and overall network for $\mu_{\mathrm{p}}=$ (a) 0.01 , (b) 0.1 , and (c) 1.0 in linear scale for the data inside the shear band. The forces are normalized by the mean tangential force of the overall network. 


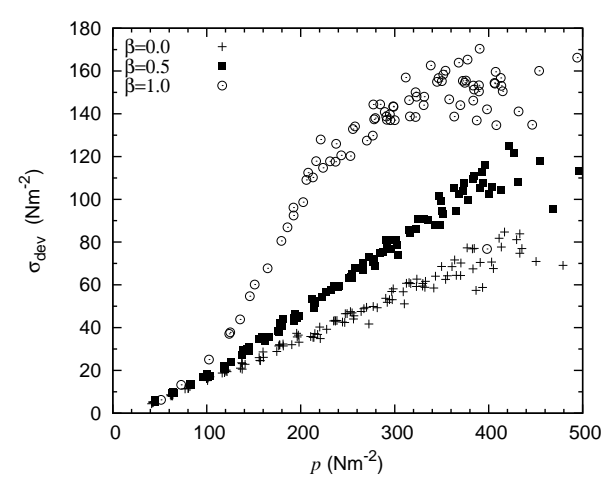

Figure 4.13: Shear stress $\sigma_{\operatorname{dev}}$ plotted against pressure $p$. Different symbols corresponds to simulations using different particle cohesion parameters $\beta$, as given in the inset.

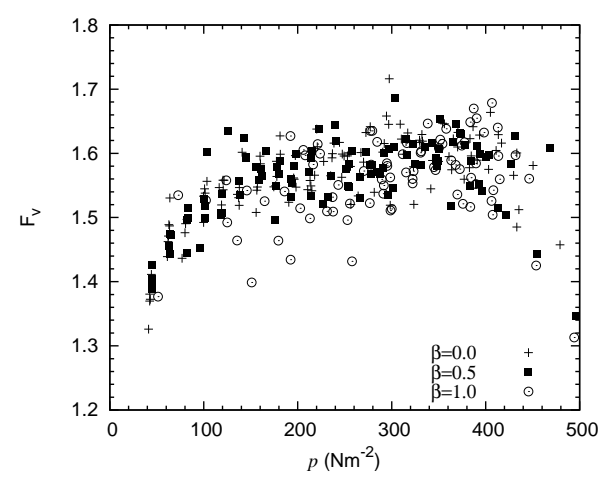

Figure 4.14: Volumetric fabric $F_{\mathrm{v}}$ plotted against pressure $p$. Different symbols corresponds to simulations using different particle cohesion parameters $\beta$, as given in the inset.

previous analysis, i.e., an increase followed by slight decrease for the highest pressure. Interestingly, for the intermediate $\beta=0.5, F_{\mathrm{dev}}$ is found to decrease with increasing $p$, differently from $F_{\mathrm{v}}$ : With the increase in cohesion, contacts redistribute themselves more isotropically, with different contact orientations, even though the total number of contacts remains almost unaffected.

For the strongest cohesion $\beta=1.0$, a different behavior is observed: $F_{\mathrm{dev}}$ first decreases with pressure, starting at low pressure where the structural anisotropy is almost unaffected by cohesion; at higher pressure, a slight increase/saturation trend is observed, even though the fluctuations are too large to conclude on this.

For cohesive particles, the strength of adhesive contact force is pressure dependent [107]. Hence the probability of loosing a contact or building up a new contact becomes pressure 


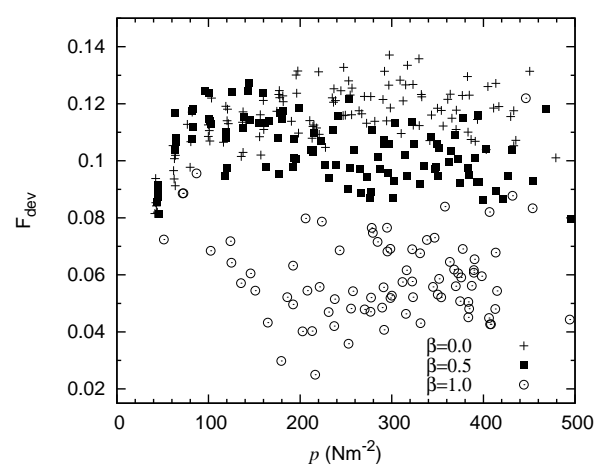

Figure 4.15: Deviatoric fabric $F_{\text {dev }}$ plotted against pressure $p$. Different symbols corresponds to simulations using different particle cohesion parameters $\beta$, as given in the inset.

dependent. With increasing cohesion, the particles will have a tendency to stick and stay together, hence less contacts are lost in tensile direction. The compressive direction is much more affected than the tensile direction, which leads to decrease in anisotropy.

\subsection{Discussion}

The effect of micro-mechanical parameters on the macroscopic flow properties of a granular material have been studied by means of the discrete element method (DEM). Different features have been highlighted, when varying contact friction and cohesion. The effect of contact friction on both the macroscopic and microscopic properties of a granular material have been studied in search of the connection between them.

In case of non-cohesive materials, the termination locus (critical state shear stress) is a linear function of pressure, as predicted by the Mohr-Coulomb criterion. While analyzing the contact network, as a split in weak and strong subnetworks, we observe that the latter carries the majority of the stress and fabric anisotropy, in agreement with previous studies [154, 195], with values increasing and then saturating with friction. Looking into the microscopic properties, we find that friction affects the probability distribution of normal and tangential forces in a very similar fashion: the increase of contact friction leads to an increase of the heterogeneity (width of the PDF) in both the force networks. However, when the focus moves on the anisotropy, i.e., the probability distributions along compressive and tensile directions, a systematic increase in anisotropy (difference between the distribution mean values along the two directions) is seen with increasing friction, but only for normal forces. Surprisingly, no similar trend is present in these directions for the tangential forces. Thus, friction influences the behavior of the force network in both its components, moving from a Gaussian-like bell-shaped distribution, with a peak around the mean, for weak friction to an 
exponential type with continuously decreasing probability with force, for stronger friction.

The relation between shear stress and pressure becomes non-linear when cohesion is introduced at the contacts. The contact network is not affected by cohesion, since contact number density remains unaffected by cohesion. The fabric anisotropy is found to decrease with cohesion. This hints at a possible redistribution of contacts along tensile and compressive directions, while keeping the total number of contacts to be unaffected. Hence cohesion affects the spatial orientation of contacts but not the total number of contacts.

A comparison of strength of force chains for different cohesion and friction deserves further investigation in the future. 


\title{
Chapter 5
}

\section{Effect of cohesion on shear banding in granular materials*}

\begin{abstract}
Very often when dealing with powder technology, a fundamental point is raised, what is the effect of contact cohesion on the bulk behavior? A dimensionless parameter to determine the intensity of cohesive forces is the granular Bond number (Bo). Using DEM simulations, we investigate the effect of contact cohesion on slowly sheared dense, dry, frictional-cohesive powders in a split bottom Couette cell. Because of the geometry a wide stable shear band forms and the steady state becomes the focus. The shear banding phenomenon is independent of cohesion for Bond number Bo $<1$ and dependent on cohesion for Bo $\geq 1$, when cohesive forces start to play an important role. Inside the shear band, we find that the mean normal contact force is independent of cohesion, while the forces carried by contacts along the (compressive and tensile) eigen-directions of the local strain rate are cohesion dependent. Forces carried by contacts along the compressive and tensile directions are symmetric about the mean force, while the force along the neutral direction is like the mean total force. The anisotropy of the force network increases with cohesion. Cohesion also increases the heterogeneous structures in both compressive and tensile directions.
\end{abstract}

*. Based on A. Singh, V. Magnanimo, K. Saitoh, and S. Luding. Effect of cohesion on shear banding in quasistatic granular material. Phys. Rev. E, Under Review, 2014 


\subsection{Introduction and Background}

Granular materials such as sand and limestone, neither behave like elastic solids nor like normal fluids, which makes their motion difficult to predict. When they yield under slow shear, the relative motion is confined to narrow regions (between large solid-like parts) called shear bands $[22,70,167]$. Shear bands are observed in many complex materials, which range from foams [83] and emulsions [68, 140] to colloids [42] and granular matter [13, 22, 54, 70, $91,92,113,116,117,125,132,172]$. There has been tremendous effort to understand the shear banding in flow of cohesionless grains $[13,22,54,64,70,91,92,113,116,117,125$, 132, 172]. However, real granular materials often experience inter-particle attractive forces because of different physical origins: van der Waals force due to atomic forces for small grains [29, 151, 210], capillary forces due to presence of humidity [65, 115], solid bridges [21], coagulation of particles [63], and many more.

The question, arises how does the presence of attractive forces affect shear banding? So far, only a few attempts have been made to answer this question, concerning dense metallic glasses [93, 187], adhesive emulsions [15, 30], cemented granular media [50], wet granular media [114, 169] and clayey soils [231]. Recently, rheological studies on adhesive and nonadhesive emulsions $[15,30,155]$ reported that the presence of attractive forces at contact affects shear banding by affecting flow heterogeneity and wall slip.

Another unique yet not completely understood feature of granular materials is their highly heterogeneous contact force distribution. The heterogeneity in the force distribution has been observed in both physical experiments $[18,70,97,98,111,124]$ and numerics $[95,154,176$, 184]. While huge effort has been made to understand the force distribution of cohesionless particles $[18,70,95,97,98,111,124,154,174]$, only limited studies have aimed to understand the same for particles with attractive interactions [56, 153, 157, 206, 210, 230]. Richefeu et al. [158] studied the stress transmission in wet granular system subjected to isotropic compression. Gilabert et al. [56] focussed on a two-dimensional packing made of particles with short-range interactions (cohesive powders) under weak compaction. Yang et al. [230] studied the effect of cohesion on force structures in a static granular packing by changing the particle size. In a previous study [107],the effect of dry cohesion at contact on the critical state yield stress was studied. The critical-state yield stress shows a peculiar non-linear dependence on the confining pressure related to cohesion. But the microscopic origin was not studied.

In this chapter, we report the effect of varying attractive forces at contact on the steady state flow behavior and the force structure in sheared dry cohesive powders. Discrete Element Method (DEM) simulations are used to investigate the system at micro (partial) and macro level. In order to quantify the intensity of cohesion, a variation of the granular Bond number $[56,128,161]$ is introduced. We find that this dimensionless number very well captures the transition from a gravity/shear-dominated regime to the cohesion-dominated regime. To 
understand this further we look at the effect of cohesion on the mean force and anisotropy, by investigating the forces along the eigen-directions of the local strain rate tensor. Intuitively, one would expect only the tensile direction to be affected by cohesion, but the real behavior is more complex. We also discuss the probability distributions and heterogeneities of the forces in different directions to complete the picture.

The chapter is organized in four main parts. Section 5.2 describes the model system in detail specifying the geometry, details of particle properties, interaction laws and the micro-macro procedure. In section 5.3, the velocity profiles and shear band from samples with different contact cohesion are presented. In the same section, the force anisotropy and probabilities are studied too. Finally, section 5.4 is dedicated to the discussion of the results, conclusions and an outlook.

\subsection{Discrete element method simulation (DEM)}

In this section, we explain our DEM simulations. We introduce a model of cohesive grains in Sec. 5.2.1 and show our numerical setup in Sec. 5.2.2. In Sec. 5.2.3, we introduce a control parameter, i.e. global Bond number, which governs the flow profiles and structure of the system.

\subsubsection{Model}

Discrete Element Methods (DEM), provide numerical solutions of Newton's equations of motion based on the specification of particle properties viz. stiffness, density, radius and a certain type of interaction laws like Hertzian/Hookean [1, 33]. Simulation methodology and material parameters used in this study are the same as in our previous work [107]. The adhesive elasto-plastic contact model [102] is used to simulate cohesive bulk flow. The model is discussed in detail in Chap. 3, and is briefly touched below.

Briefly, in the adhesive elasto-plastic contact model, three physical phenomena: elasticity, plasticity and adhesion are quantified by three material parameters $k_{p}, k_{1}$, and $k_{c}$, respectively. $k_{1}$ together with $k_{p}$ and $k_{c}$ signifies the intensity of plastic dissipation, and adhesion force respectively. Plasticity disappears for $k_{1}=k_{p}$ and adhesion vanishes for $k_{c}=0$. The hysteretic force is introduced by allowing the un- and re-loading stiffness $k_{2}$ to depend on history of the deformation, which interpolates linearly between these two extremes $\left(k_{1}\right.$ and $k_{p}$ ) (for details see Eq. 1 in [102]). The overlap $\left(\delta_{0}\right)$ when the unloading force reaches zero, resembles the permanent plastic deformation and depends nonlinearly on the previous maximal force. The negative forces reached by further unloading are attractive, adhesion forces, which also increase nonlinearly with the previous maximal compression force experienced. The maximal adhesion force is given by

$$
f_{\mathrm{m}}=-k_{c} \delta_{\min }
$$


with $\delta_{\min }=\frac{k_{2}-k_{1}}{k_{2}+k_{c}} \delta_{\max }$.

Three physical phenomena: elasticity, plasticity and cohesion are quantified by three material parameters $k_{p}, k_{1}$, and $k_{c}$, respectively. Plasticity disappears for $k_{1}=k_{p}$ and cohesion vanishes for $k_{c}=0$.

The contact friction is set to $\mu=0.01$, i.e. artificially small, in order to be able to focus on the effect of contact cohesion only. In order to study the influence of contact cohesion, we analyzed the system for the following set of adhesivity parameters $k_{c}$ :

$$
k_{c} \in[0,5,10,25,33,50,75,100,200] \mathrm{Nm}^{-1},
$$

which has to be seen in relation to $k_{1}=100 \mathrm{Nm}^{-1}$. Other parameters, such as $f_{a}$ [178] and $\phi_{f}[102,178]$ are not changed here.

\subsubsection{Split-bottom ring shear cell}

We perform numerical simulation in the same setup described earlier in Sec. 2.1.1.1. It consists of two concentric cylinders, with inner and outer cylinder radii as $R_{i}=0.0147 \mathrm{~m}$ and $R_{O}=0.11 \mathrm{~m}$ respectively, with a split radius being $R_{S}=0.085 \mathrm{~m}$ (as shown in Fig. 2.2). The concentric cylinders rotate relative to each other around the symmetry axis (the dotdashed line).

The ring shaped split at the bottom separates the moving and static parts of the system, where a part of the bottom and the outer cylinder rotate at the same rate. The system is filled with $N \approx 3.7 \times 10^{4}$ spherical particles with density $\rho=2000 \mathrm{~kg} / \mathrm{m}^{3}=2 \mathrm{~g} / \mathrm{cm}^{3}$ up to height $H$. The cylindrical walls and the bottom are roughened due to some (about $3 \%$ of the total number) attached/glued particles, as explained in [103-105]. When there is a relative motion at the split, a shear band propagates from split position $R_{S}$ upwards and inwards and remains far away from cylinder-walls and bottom in most cases. Since we are interested in the quasi-static regime, the rotation rate of outer cylinder is chosen to be $\frac{\Omega}{2 \pi}=0.01 \mathrm{~s}^{-1}$, such that the inertial number $I \ll 1$ [118]. The simulation runs for more than $50 \mathrm{~s}$.

\subsubsection{Dimensionless number}

Intensity of cohesion can be quantified by a ratio of the maximum attractive force to a typical force scale in the system. For example, Nase et al. [128] introduced the granular Bond number under gravity, which compares the maximum attractive force at contact with the weight of a single grain. For plane shear without gravity, others $[56,161]$ used a ratio between the maximum attractive force and the average force due to the confining pressure. In our analysis, we introduce a global Bond number as

$$
\text { Bo }=\frac{f_{\mathrm{m}}}{\langle f\rangle},
$$


where $f_{\mathrm{m}}$ and $\langle f\rangle$ are the maximum allowed attractive force reached at a contact (given by the contact model, Eq. (5.1)) and the mean force per contact reached close to the bottom, respectively. For the calculation of mean force $\langle f\rangle$, layer of two particles diameters which is few particle diameter away from bottom is chosen. Because the shear band initiates from the bottom, we choose the mean force $\langle f\rangle$ at the bottom to understand the effect of cohesion on these shear bands.

It is important to mention that the mean compressive force (at the bottom) corresponds to the weight of the material above, whereas the maximum attractive force corresponds to the pull-off force, which is directly related to the surface energy of the particles. These two material and particle properties are easily accessible experimentally.

The Bond number is a measure of the importance of adhesive forces compared to compressive forces. A low Bond number indicates that the system is relatively unaffected by attractive force effects; a high number (typically larger than one) indicates that attractive forces dominate. Intermediate numbers indicate a non-trivial balance between the two effects.

In parallel with the global Bond number as defined above, we also define two variants of this quantity locally. A local simulation based Bond number $B o_{l}^{s}(P)=f_{\mathrm{m}}^{s}(P) /\langle f(P)\rangle$ can be define by comparing the maximum attractive force reached at a given pressure (which can be less than or equal to the maximum allowed attractive force given by contact model) with the mean force at that pressure (subscript $l$ represents the local quantity, while superscript $s$ denotes that this definition takes input from simulation data). Another variant of this $B o_{l}^{a}(P)$ is defined in Appendix, which compares analytical prediction for maximum attractive force with mean force at that pressure.

Figure 5.1 displays the global Bond number $B o$ and the mean values of $B o_{l}^{s}(P)$ and $B o_{l}^{a}(P)$ (averaged over different pressure) as functions of the adhesivity parameter $k_{c}$, where they are comparable with each other. For the sake of simplicity in the rest of this chapter, we use the global Bond number $B o$ to quantify the intensity of cohesion.

\subsection{Results}

In this section, we present our results of DEM simulations. In Sec. 5.3.1, we analyze the flow profiles and shear banding in our system. In Sec. 5.3.2, we study distributions and structures of force chain networks in shear bands. In Sec. 5.3.3, we explain anisotropic features of the force chain networks.

\subsubsection{Effect of cohesion on the flow profiles}

Figure 5.2 displays both top- and front-view of samples with the same filling height, i.e. the same number of particles, and different global Bond numbers, $B o=$ (left) 0 and (right) 4.86, 


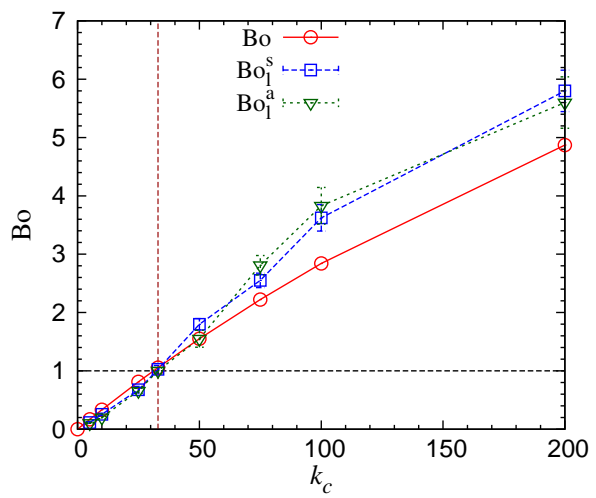

Figure 5.1: (Color online) Variants of granular Bond number plotted against cohesive strength $k_{c}$, where the red circles represent the global Bond number $B o$, while the blue squares and green triangles represent the average values of $B o_{l}^{s}(P)$ and $B o_{l}^{a}(P)$, respectively.

respectively, where the color code represents the azimuthal displacement rate of particles. From the front-view of particles, the shear bands (green colored area) move inwards and get wider with increasing "height", while the shear band also moves inwards and becomes wider with increasing "cohesion".

Figure 5.3 shows the non-dimensional angular velocity profiles at the top surface against radial coordinate normalized with mean particle diameter $\langle d\rangle$, where we assume translational invariance in the azimuthal direction and take averages over the toroidal volumes as well as many snapshots in time [91]. The angular velocity profile can be well approximated by an error function

$$
\omega=A_{1}+A_{2} \operatorname{erf}\left(\frac{r-R_{c}}{W}\right)
$$

as in the case of non-cohesive materials $[43,52,54,103,105]$, where $R_{c}$ and $W$ are the position and width of the shear band, respectively. Here, we use the dimensionless amplitudes, $A_{1}=A_{2} \approx 0.5$, for the whole range of the global Bond numbers, while we use $A_{1}=0.58$ and $A_{2}=0.42$ for the strong cohesion with $B o=4.86$. We summarize the dimensionless amplitudes, $A_{1}$ and $A_{2}$, in Table 5.1. Figure 5.4 plots the position of shear band relative to the split at bottom $R_{S}-R_{c}$ and the width of shear band $W$ at the top surface against the global Bond number. Here, within the error-bars, both the position and width are independent of cohesion if $B o<1$. However, the shear band moves inside and becomes wider with the global Bond number if $B o>1$.

Both the position and width of shear band also depend on the height $(z)$ in the system. Figure 5.5 displays the non-dimensional position and width of the shear band for different values of $B o$ as functions of the height scaled by the filling height, i.e. $z / H$. In this figure, the shear band moves closer to the inner cylinder and gets wider while approaching to the top layer, 

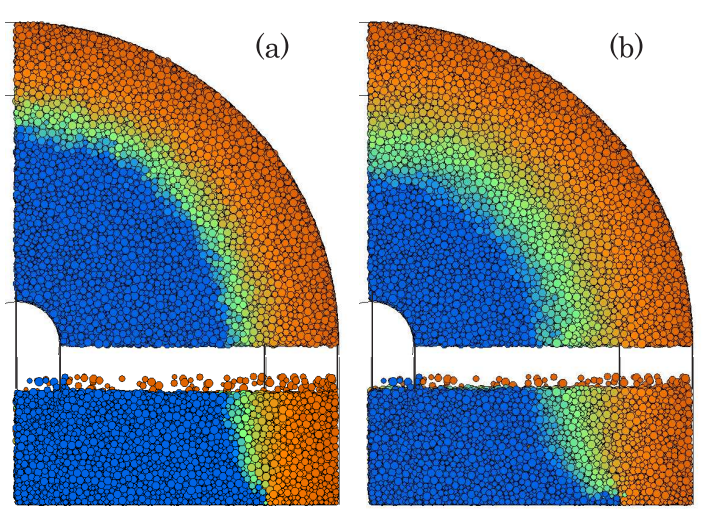

Figure 5.2: (Color online) Snapshots from simulations with different cohesion strengths, but the same number of mobile particles $N=34518$, seen from the top (Top) and from the front (Bottom). The material is (a) without cohesion $B o=0$, and (b) with strong cohesion $B o=4.86$. The colors blue, green, and orange denote the particles with displacements in tangential direction per second $r \mathrm{~d} \phi \leq 0.5 \mathrm{~mm}, r \mathrm{~d} \phi \leq 2 \mathrm{~mm}, r \mathrm{~d} \phi \leq 4 \mathrm{~mm}$, and $r \mathrm{~d} \phi>4 \mathrm{~mm}$, respectively

which is consistent with the previous cohesive and non-cohesive studies [43, 52, 54, 103, 105, 107, 159]. In Fig. 5.5a, the lines are the prediction by Unger et al. [209]:

$$
z=H-R_{c}\left\{1-\frac{R_{s}}{R_{c}}\left[1-\left(\frac{H}{R_{S}}\right)^{\beta}\right]\right\}^{1 / \beta},
$$

where the exponent is given by $\beta=2.5$ for cohesionless particles. If the global Bond number is less than one, our numerical data show very good agreement with Eq. (5.5). Above $B o=1$, however, the exponent $\beta$ decreases with the global Bond number as in Table 5.1. Note that Eq. (5.5) slightly deviates from the results near the top surface if the cohesion is strong $(B o=2.22$ and 2.85). In Fig. 5.5b, the lines are the prediction by Ries. et al. [159]:

$$
W(z)=W_{\text {top }}\left[1-\left(1-\frac{z}{H}\right)^{2}\right]^{\gamma},
$$

where $W_{\text {top }}$ is the width at the top surface and the exponent is given by $\gamma=0.5$ for cohesionless particles. If $B o<1$, Eq. (5.6) with $W_{\text {top }}=0.012$ and $\gamma=0.5 \pm 0.1$ well agrees with our results. However, if $B o>1$, both the width $W_{\text {top }}$ and exponent $\gamma$ increase with the global Bond number as in Table 5.1. In addition, Eq. (5.6) deviates from the results near the top layer if the cohesion is strong $(B o=2.22$ and 2.85), where the width initially increases with the height, but saturates above $z / H \simeq 0.6$. Hence for $B o>1$, we choose width at that height to be $W_{\text {top }}$ and use $\gamma=0.66$ and 0.7 for $B o=2.22$ and 2.85 , respectively.

From the above results, we find that the cohesive forces between particles drastically affect the flow profiles. Eqs. (5.5) and (5.6) very well predict the position and width of the 
shear bands, respectively for $B o<1$. For large $B o$ these equations do not work anymore at large heights since the shear band interferes with the inner cylinder. The shear band, which is a large velocity gradient, is caused by sliding motions of particles. However, cohesive forces strongly connect the particles in contacts (in other words, the cohesive forces promote collective motions of particles) and prevent them from sliding. As a result, the velocity gradient is smoothed and the width of shear-band is broadened. This observation is consistent with previous studies on adhesive dense emulsions [141]. Interestingly, such an effect of cohesion is suppressed if the global Bond number is less than one, where our numerical data agrees well with previous theoretical/numerical studies on non-cohesive particles [159, 209]. Hence, we show that the global Bond number, $B o$, captures the transition between essentially non-cohesive free-flowing granular assemblies $(B o<1)$ to cohesive ones $(B o>1)$.

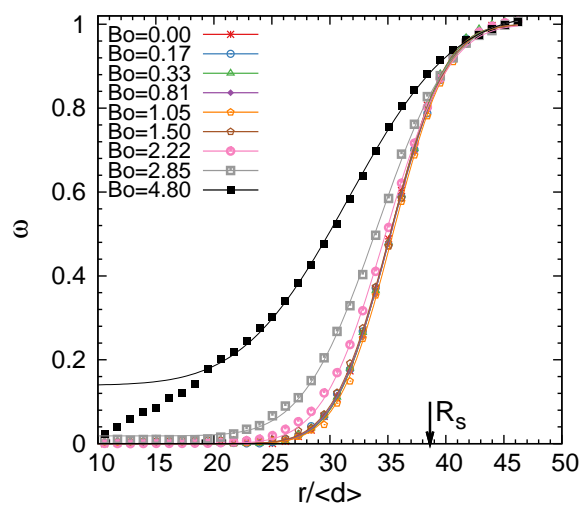

Figure 5.3: (Color online) Non-dimensional angular velocity profile $\omega$ at the top surface plotted against the radial coordinate $r$ scaled by the mean diameter $\langle d\rangle$. Different symbols represent different values of the global Bond number given in the inset, where the solid lines represent the corresponding fits to Eq. (5.4).

\subsubsection{Structure and distribution of forces in shear bands}

To understand the microscopic origin of the anomalous flow profiles of cohesive aggregates, we study structures of force chains and statistics of the interparticle normal contact forces. Recently Wang et al. [224] reported the shape of probability distribution as an indicator for transition of flow from quasistatic to inertial flows. In this section, we us similar philosophy to determine if there is any change in the shape of PDFs as the cohesive strength is increased.

Figure 5.6 shows force chains of positive ((a) and (b)) and negative ((c) and (d)) normal forces in the systems with low cohesion ((a) and (c)) and strong cohesion ((b) and (d)). Grey color shows the weak forces, while red and blue colors show the strong positive and negative forces respectively. The strong or weak positive forces are forces larger or smaller 


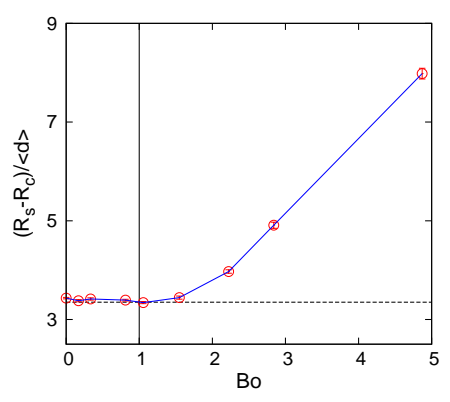

(a)

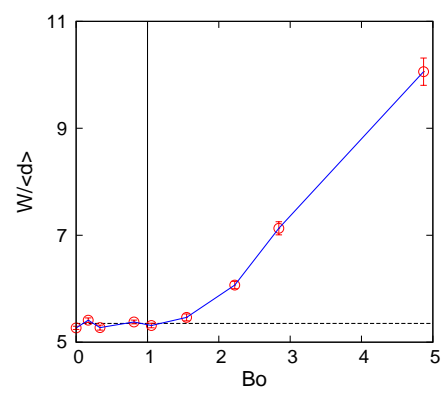

(b)

Figure 5.4: (Color online) (a) Position and (b) width (both scaled by mean particle diameter) of shear band at the top surface plotted against the global Bond number. Symbols with error-bars are the data, while the lines are only a guide to eye.

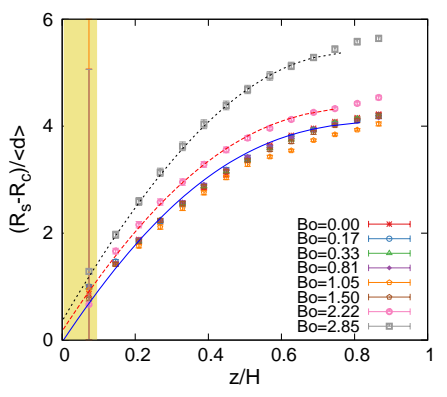

(a)

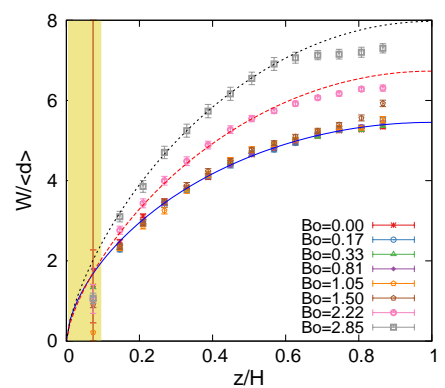

(b)

Figure 5.5: (Color online) (a) Position and (b) width (both scaled by mean particle diameter) of shear band in the cell plotted against height $z$ scaled by the filling height $H$. Different symbols correspond to values of the global Bond number given in the inset. The lines in (a) and (b) are the predictions, Eqs. (5.5) and (5.6), respectively.

than the mean positive force $f_{\text {pos }}$. A similar approach is adopted to identify the strong/weak negative forces. In this figure, we observe that both the positive and negative forces are fully developed in the cohesive system ((b) and (d)), where the intensity of the positive/negative force inside of the shear band is much stronger than that outside. In addition, the strong (positive/negative) force chains are percolated through the shear band region. As explained in Sec. 5.3.3, we can also see that the positive and negative force chains are aligned in their preferred directions, i.e. compressive and tensile directions, respectively.

Figure 5.7 displays scatter plots of the interparticle forces against overlaps between the particles in contacts, where each point corresponds to a contact and different colors represent different height, i.e. pressure level in the system. The left and right columns are the results 


\begin{tabular}{|l|l|l|l|l|l|l|l|}
\hline Bo & $A_{1}$ & $A_{2}$ & $H$ & $\beta$ & $\frac{z}{H}$ range & $W_{\text {top }}$ & $\gamma$ \\
\hline 0 & $0.50 \pm 0.0005$ & $0.500 \pm 0.0005$ & 0.0365 & 2.52 & $0.1-1$ & 0.0117 & 0.507 \\
0.17 & $0.50 \pm 0.0005$ & $0.499 \pm 0.0005$ & 0.0365 & 2.52 & $0.1-1$ & 0.0118 & 0.523 \\
0.33 & $0.49 \pm 0.0007$ & $0.500 \pm 0.0007$ & 0.0365 & 2.512 & $0.1-1$ & 0.0118 & 0.555 \\
0.81 & $0.49 \pm 0.0008$ & $0.500 \pm 0.0008$ & 0.0361 & 2.494 & $0.1-1$ & 0.0119 & 0.583 \\
1.05 & $0.49 \pm 0.001$ & $0.501 \pm 0.001$ & 0.0359 & 2.510 & $0.1-1$ & 0.0120 & 0.582 \\
1.50 & $0.49 \pm 0.002$ & $0.501 \pm 0.002$ & 0.0364 & 2.453 & $0.1-0.8$ & 0.0126 & 0.613 \\
2.22 & $0.49 \pm 0.003$ & $0.501 \pm 0.003$ & 0.0368 & 2.367 & $0.1-0.6$ & 0.0138 & 0.667 \\
2.85 & $0.49 \pm 0.005$ & $0.502 \pm 0.005$ & 0.0369 & 2.259 & $0.1-0.6$ & 0.0160 & 0.713 \\
\hline
\end{tabular}

Table 5.1: Table showing filling height of the system $H$, and fitting range $z / H$ for Eqs. (5.5) and (5.6), together with the fit parameters $A_{1}, A_{2}$ in Eq. (5.4), $\beta$ in Eq. (5.5), $W_{\text {top }}$ and $\gamma$ in Eq. (5.6).

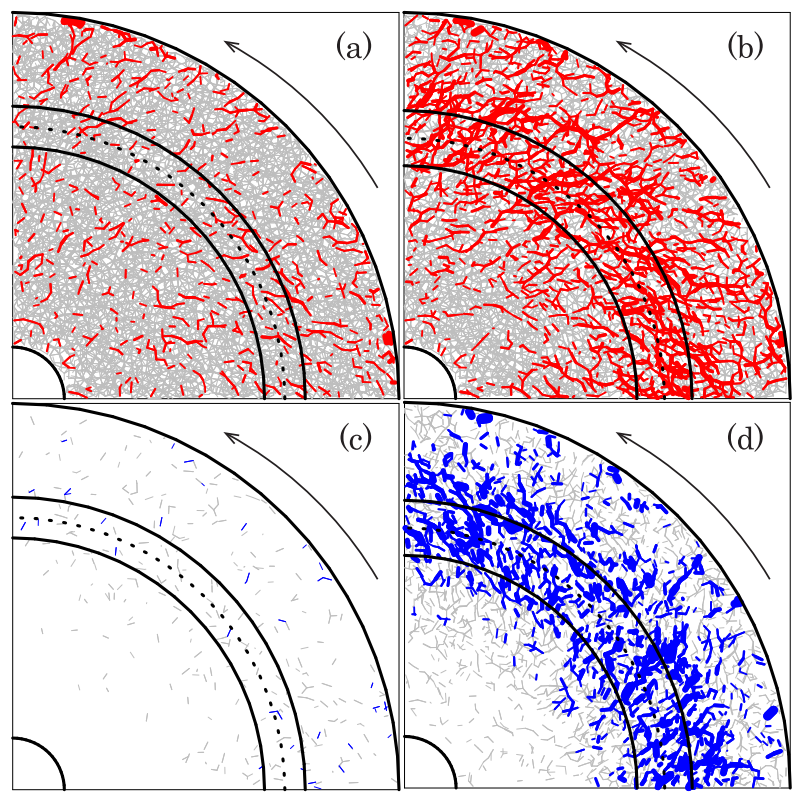

Figure 5.6: (Color online) Force chain networks of positive normal forces for $B o=(\mathrm{a})$ 0.33 and (b) 2.85, and negative normal forces for $B o=$ (c) 0.33 and (d) 2.85 at height $0.02<z<0.05 \mathrm{~m}$, respectively.

of inside and outside of shear bands, respectively. The lower $z$ is, the higher is the average force (or overlap), to sustain a pressure due to the weight of the particles. For almost all values of $B o$, the density of points towards unloading $k_{p}$ branch inside the shear band is higher compared to the points outside. We also observe that with increasing $B o$, the most contacts (except for small pressure) drift towards and collapse around the limit branch of the contact 

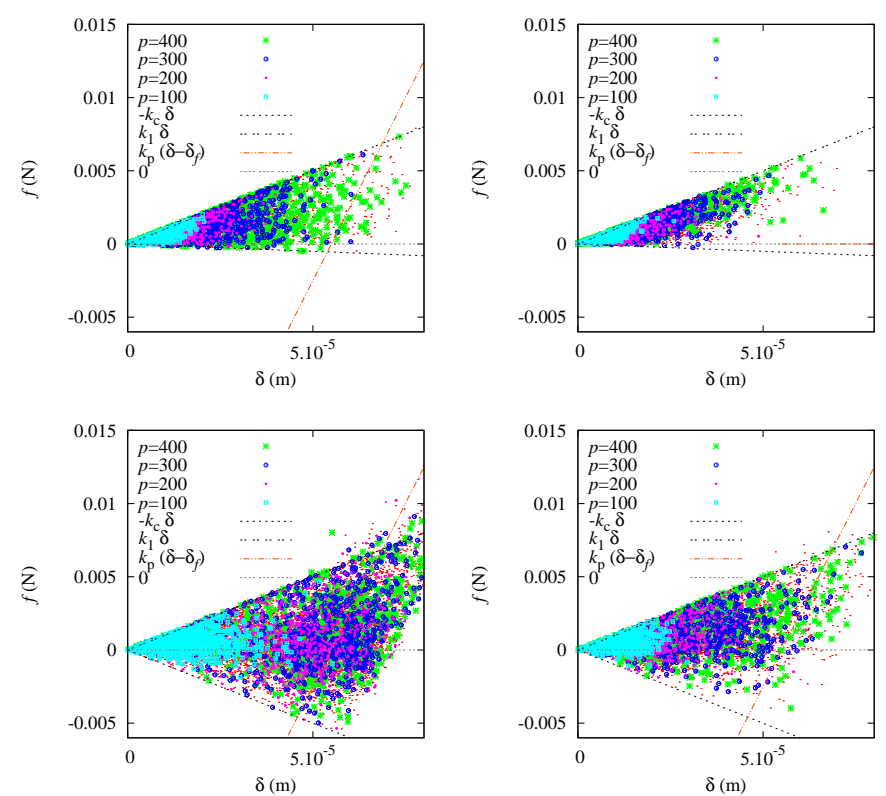

Figure 5.7: (Color online) Scatter plots of overlaps and forces between all contacts inside (left) and outside (right) of the shear bands for different $B o=0.33$ and 2.85. The different symbols represent a zoom into the vertical ranges $z=8 \mathrm{~mm} \pm 1 \mathrm{~mm}$ (green stars), $15 \mathrm{~mm}$ $\pm 1 \mathrm{~mm}$ (blue circles), $22 \mathrm{~mm} \pm 1 \mathrm{~mm}$ (magenta dots), $29 \mathrm{~mm} \pm 1 \mathrm{~mm}$ (cyan squares), with approximate pressure as given in the inset. Note that the points do not collapse on the line $k_{p}\left(\delta-\delta_{f}\right)$ due to the finite width of the size distribution: pairs of larger than average particles fall out of the indicated triangle. Radial range $0.075 \mathrm{~m} \leq r \leq 0.085 \mathrm{~m}$ signifies data points inside the shear band, while the radial range $0.055 \mathrm{~m} \leq r \leq 0.065 \mathrm{~m}$ signifies the data points outside the shear band.

model (especially inside shear band). This implies, the cohesive forces are more pronounced in shear bands rather than the outside.

\subsubsection{Mean force and overlap in shear bands}

Figure 5.8 displays the mean normal forces, $\langle f\rangle$, in shear bands against pressure, $P$, for different values of the global Bond number, where the solid line is the prediction by Shaebani et al. [173] for non-cohesive granular systems as

$$
\langle f\rangle=\frac{4 \pi\left\langle a^{2}\right\rangle}{\phi C g_{2}}\langle P\rangle
$$

with the $2^{\text {nd }}$ moments of size distribution $\left\langle a^{2}\right\rangle$, coordination number $C$, volume fraction $\phi$, and mean pressure $\langle P\rangle$. Notably, the mean normal force is almost independent of cohesion 


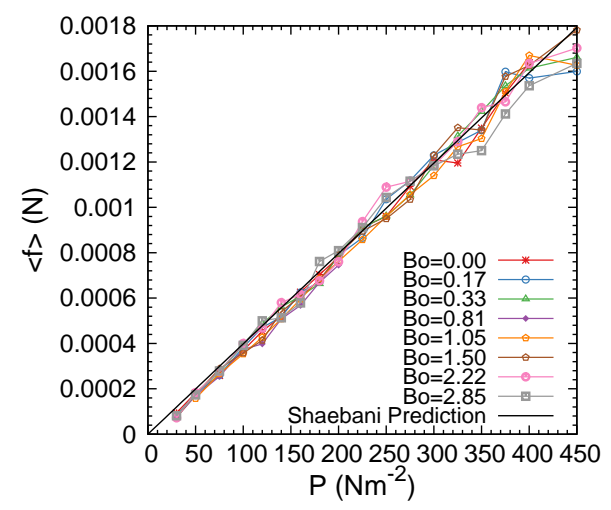

Figure 5.8: (Color online) The mean normal force inside of the shear band plotted against pressure, where different symbols represent the global Bond number (as given in the inset) and the solid line is given by Eq. (5.7).

and linearly increases with pressure as in the cases of static non-cohesive $[124,176]$ and cohesive systems [230]. We also observe that for low pressure, Eq. (5.7) slightly over predicts the value of the mean force, while for higher pressure the prediction well captures the data.

While the mean value is insensitive to cohesion, the mean positive and negative normal forces, $\left\langle f_{\text {pos }}\right\rangle$ and $\left\langle f_{\text {neg }}\right\rangle$, strongly depend on cohesion. Figure 5.9 shows the mean positive and negative forces against pressure for different values of $B o$, where the intensities of the mean positive and negative forces increase with cohesion as we observed the fully developed positive/negative force chains in the cohesive system (Fig. 5.6). Note that the mean positive force is linear against pressure and independent of cohesion below $B o=1$, while its dependence on pressure becomes nonlinear above $B o=1$. Though the origin of this nonlinearity is not clear, it is readily understood that cohesion enhances the collective motion of the particles, i.e. the particles rearrange less and the system is in a mechanically constrained state. Because the increase of cohesion also increases the magnitude of negative forces, both the positive and negative force chains remain strong to balance each other. It is noteworthy that in Fig. 5.7, the increase of $B o$ increases the density of points in both positive and negative extremes, inside the shear band.

The cohesive force seems not to affect the average number of contacts, see Ref. [179], where we reported that cohesion had practically no effect on the contact number density (volumetric fabric) in the same system. Fig. 5.10 shows the fractions of repulsive and attractive contacts against pressure for different Bond numbers, together with the overall coordination number. An increase of cohesion generates more attractive contacts while it decreases the number of repulsive contacts. Interestingly, the overall mean force remains independent of cohesion and contacts simply redistribute between the repulsive and attractive directions. 


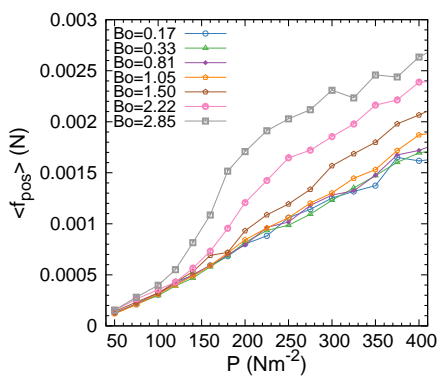

(a)

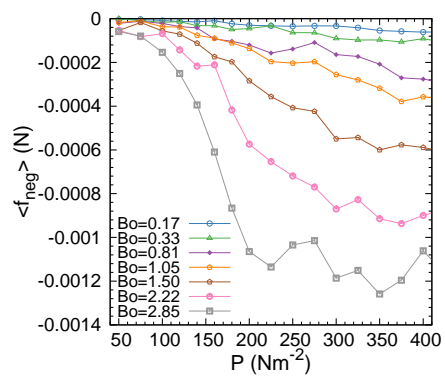

(b)

Figure 5.9: (Color online) The mean (a) positive and (b) negative forces inside the shear band plotted against pressure, where different symbols represent the global Bond number (as given in the inset).

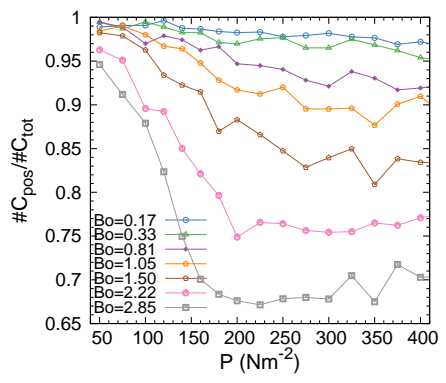

(a)

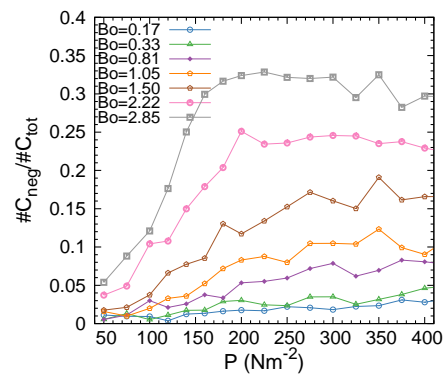

(b)

Figure 5.10: (Color online) The fractions of (a) positive and (b) negative contacts inside the shear band plotted against pressure, where different symbols represent the global Bond number (as given in the inset). 


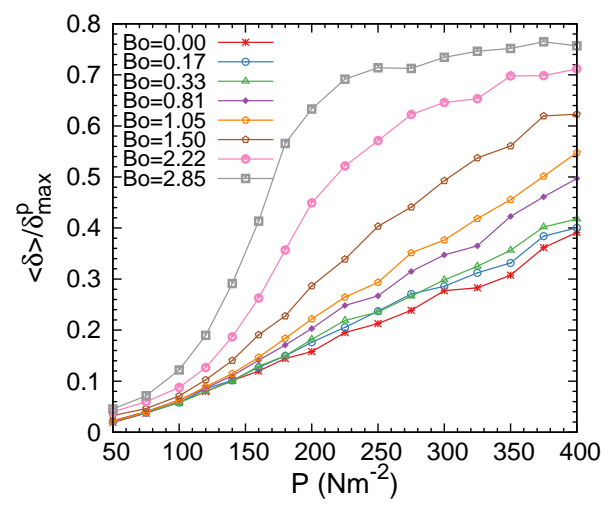

Figure 5.11: (Color online) Normalized mean overlap $\frac{<\delta>}{\delta_{\max }^{p}}$ inside the shear band plotted against pressure, where different symbols represent the global Bond number (as given in the inset).

In contrast to the mean force, the mean overlap between particles in contact depends on cohesion non-linearly as shown in Fig. 5.11. In our model of cohesive particles [102], overlaps are positive for both positive and negative forces. It is worth mentioning that for low $B o$, the time evolution of $\langle\delta\rangle$ saturates quickly, while for $B o=1.5,2.22$ it takes longer to longer to reach the steady state due to the plastic increase of the overlap in average [107].

\subsubsection{PDFs of forces and structures of strong force chains in shear bands}

The distributions of forces are also strongly affected by cohesion. Figure 5.12 shows the PDFs of normal forces in shear bands for different pressure and cohesion, where the forces are scaled by the mean normal force, i.e. $f^{*} \equiv f /\langle f\rangle$. As can be seen, the PDF in shear band for cohesion-less particles is almost independent of pressure (Fig. 5.12a), while it depends on pressure if the cohesive forces are very strong (Fig. 5.12b). Figure 5.13 displays the variations of the PDFs for different intensities of cohesion, where we find that the PDF becomes broad with increasing cohesion and this trend is more pronounced for $B o>1$. Therefore, the strong cohesion, which leads the system to "mechanically frustrated state", induces large fluctuations of positive/negative forces. We note that Yang et al. [230] also found similar trends in static three-dimensional packings for small sized particles, where the PDF becomes broader, as particle size decreases, i.e. cohesion increases. Broadening of the PDFs was also observed by Luding et al. [110] during cooling down of a sintered system.

The cohesive forces change not only the shapes of the PDFs, but also the asymptotic behaviors of the PDFs, i.e. the structures of strong force chains. At first, we fit their tails by a 


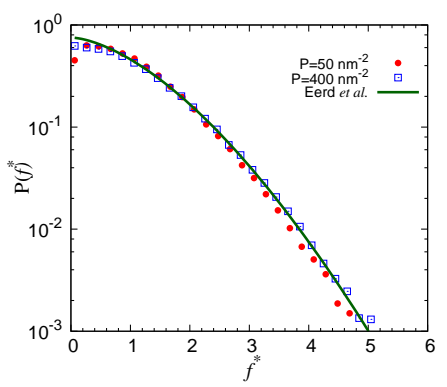

(a)

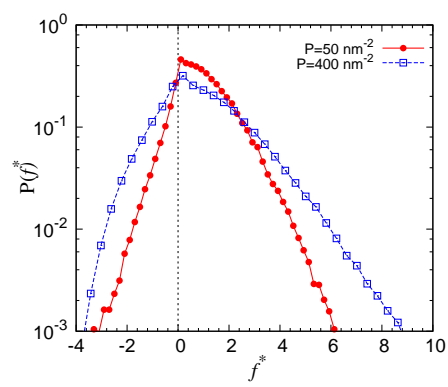

(b)

Figure 5.12: (Color online) Probability distribution of the normalized force for (a) cohesionless $B o=0$ and (b) highly cohesive $B o=2.85$ systems at different pressures in the system. Different symbols represent value of local pressure (as given in the inset).

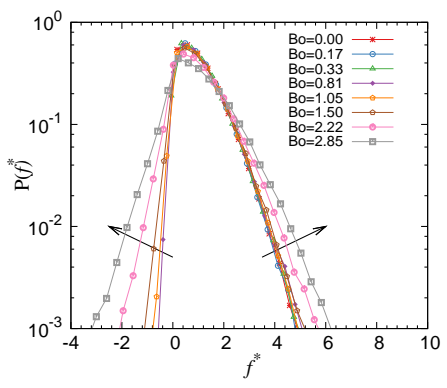

(a)

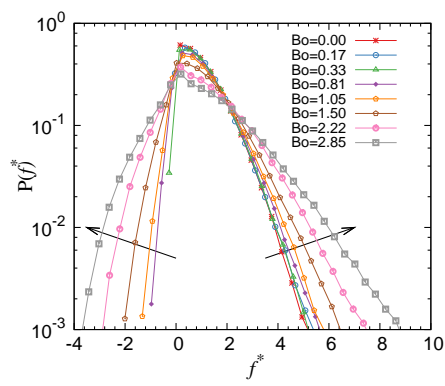

(b)

Figure 5.13: (Color online) Probability distribution of normalized force $f^{*}$ for (a) low pressure $p=50 \mathrm{Nm}^{-2}$ (close to top) and (b) high pressure $p=400 \mathrm{Nm}^{-2}$ (close to bottom) in the system for data inside the shear band. Different symbols represent the global Bond number $B o$ (as given in the inset). 


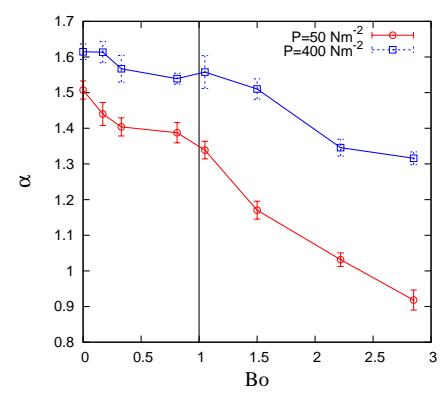

(a)

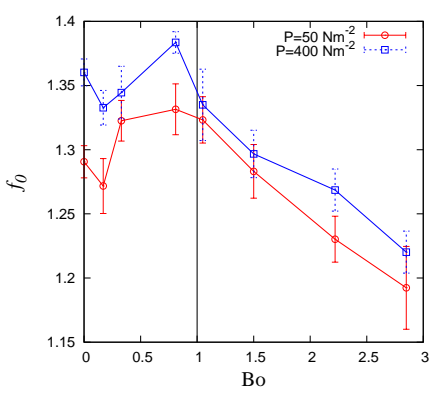

(b)

Figure 5.14: (Color online) Fit parameters (a) $\alpha$ and (b) $f_{0}$ plotted against Bond number $B o$. Different symbols represent value of local pressure (as given in the inset).

stretched exponential function [212]

$$
P\left(f^{*}\right) \sim e^{-\left(f^{*} / f_{0}\right)^{\alpha}}
$$

with a characteristic force $f_{0}$ and a fitting exponent $\alpha$. Figure 5.14 displays the characteristic force and the exponent against the global Bond number $B o$. If $B o<1$, we obtain $f_{0}=$ $1.4 \pm 0.1$ and $\alpha=1.6 \pm 0.1$, which is very close to that predicted by Eerd et al. [212] for three-dimensional cohesionless ensemble generated by MD simulations. For Bo $>1$, however, both the characteristic force and fitting exponent decrease with increasing cohesion. The decreasing fitting exponent hints at stronger fluctuations in the force distribution. A Gaussian tail of the probability distribution would indicate a more homogeneous random spatial distribution of forces. The deviation towards an exponential distribution can be linked to an increase in heterogeneity in the spatial force distribution; as mentioned in previous studies $[112,152,233]$. Therefore, we conclude that the tail of the PDF becomes more exponential with increasing cohesion, which implies a heterogeneous spatial distributions of strong forces.

Also we observe that the fitting exponent decreases with increasing pressure, which again implies that at high pressure spatial distribution is more heterogeneous compared to that for low pressure.

\subsubsection{Anisotropy of force chain networks in shear bands}

In the case of simple shear, there are two non-zero eigenvalues of the strain rate tensor, which are equal in magnitude but opposite in sign, and the third eigenvalue is zero. The plane containing the eigen-vectors with non-zero eigenvalues is called the "shear plane", where the eigen-vector with zero eigenvalue is perpendicular to this plane (parallel to the shear band). We call the eigen-directions with positive, negative, and zero eigenvalues as the 


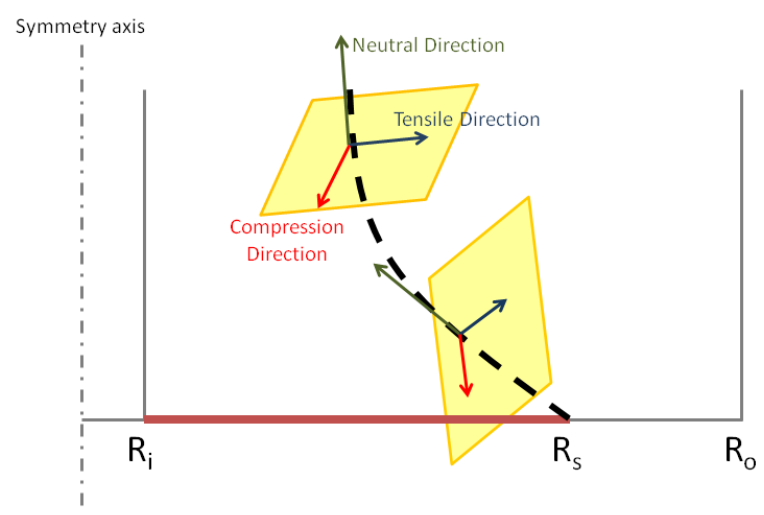

Figure 5.15: (Color online) A sketch showing the shear band as dotted line, shear plane, and three eigen-directions of the strain rate tensor. Grey lines show inner and outer cylinders, while solid brown line shows the split, dashed black line shows the shear band which initiates at the split at bottom and moves towards inner cylinder as it moves towards the top. Green arrow represents the eigen-direction for neutral eigenvalue of the strain rate tensor, which is tangential to the shear band, perpendicular to this vector is the shear plane (yellow shaded region), which contains the eigen-directions for compression (red arrow) and tensile (blue arrow) eigenvalues.

compressive, tensile, and neutral directions, respectively. Since the compressive and tensile directions are associated with loading and unloading of contacts, respectively, it is intuitive that in the absence of any external force, the mean force would be positive in compressive direction, negative in tensile direction, and almost zero in neutral direction.

In our system, both compressive forces and shear play a combined role, where the neutral direction gets a contribution from external compressive force only, while the two principal (compressive and tensile) directions get contributions from both shear and external compressive force. Because the cohesive force is activated by unloading, it should affect the force along the tensile direction. Note that the shear band here is not vertical, instead its orientation changes with depth as shown in the schematic in Fig. 5.15. In this figure, the eigen-direction of the neutral (zero) eigenvalue (green arrow) moves with the shear band. This turning of the neutral eigen-direction makes the shear plane tilt as well (which is shown by the yellow shaded regions). To extract the contacts aligned along these directions at a given pressure in the system, we first calculate the local strain rate tensor and extract the three eigen-directions $\mathbf{n}_{\gamma}$. Next, we look for contacts with unit contact vector $\mathbf{n}_{\mathrm{c}}$, which satisfy the condition $\left|\mathbf{n}_{\mathrm{c}} \cdot \mathbf{n}_{\gamma}\right|$ $\geq 0.9$. The contacts which satisfy the condition for compressive eigen-direction are termed compressive, and tensile and neutral contacts are defined similarly. The forces carried by compressive, tensile, and neutral contacts are denoted by $f_{\text {com }}, f_{\text {ten }}$, and $f_{\text {neu }}$ respectively. 


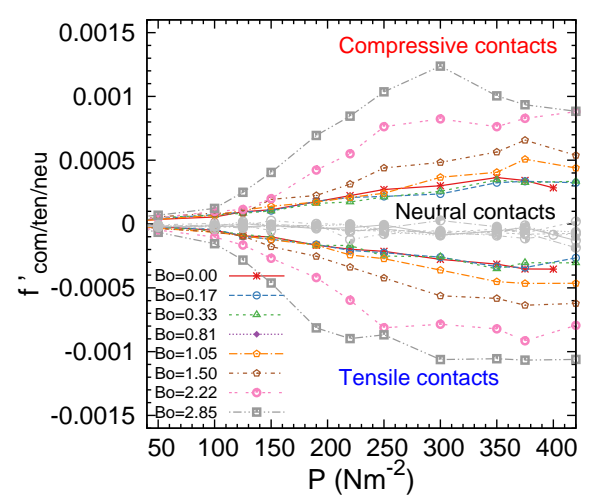

Figure 5.16: (Color online) Difference between the mean forces in different eigen-directions of the strain rate tensor subtracted from overall mean force plotted against local pressure in the system. Different symbols represent the global Bond number Bo (as given in the inset).

Figure 5.16 shows the mean forces relative to overall local mean force, $f_{\text {com } / \text { ten } / \text { neu }}^{\prime} \equiv$ $\left\langle f_{\text {com } / \text { ten } / \text { neu }}\right\rangle-\langle f\rangle$, plotted against pressure for different values of Bo. We find that $f_{\text {com }}^{\prime}(>$ 0 ) and $f_{\text {ten }}^{\prime}(<0)$ are symmetric about zero, and $f_{\text {neu }}^{\prime} \simeq 0$. Because the mean force along the neutral direction is independent of $B o$, the cohesion does not affect the neutral direction (due to the absence of shear in this direction). However, $f_{\text {ten }}^{\prime}$ decreases with pressure and cohesion, while $f_{\text {com }}^{\prime}$ increases to keep the mean overall force to stay independent of cohesion. Both positive and negative forces are present in all directions. However, the positive and negative forces dominate in the compressive and tensile directions, respectively. The anisotropy of forces is more pronounced with increasing pressure and cohesion, as observed in Fig. 5.6.

Next, we study the PDFs of forces in the compressive, tensile, and neutral directions. Figure 5.17 displays the PDFs along each direction for non-cohesive $B o=0$ and highly cohesive $B o=2.85$ systems, where the forces along different directions are normalized by the overall mean force. In a non-cohesive system (Fig. 5.17a), we observe that for $f^{*}<1$, the PDF along the tensile direction is higher compared to that for the compressive direction, which is intuitive as the majority of contacts will have smaller forces in the tensile direction. For $f^{*}>1$, however, the PDF along the compressive direction is higher compared to that along the tensile direction, as force along the compressive direction should be stronger compared to that along the tensile direction [199]. For a highly cohesive system (Fig. 5.17b), a similar behavior is observed for positive forces, while for small positive and negative forces, due to attractive forces the probability is higher along the tensile direction compared to the compressive direction. The PDFs of forces in the neutral direction lie in between those in compressive and tensile directions, suggesting a close to average distribution of forces in the neutral direction. 


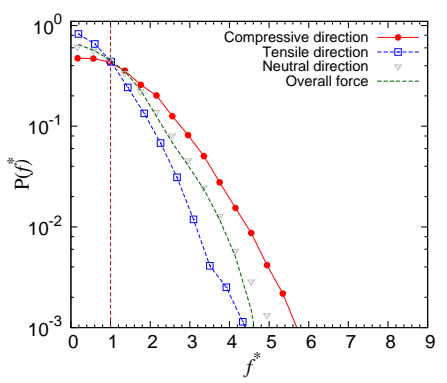

(a)

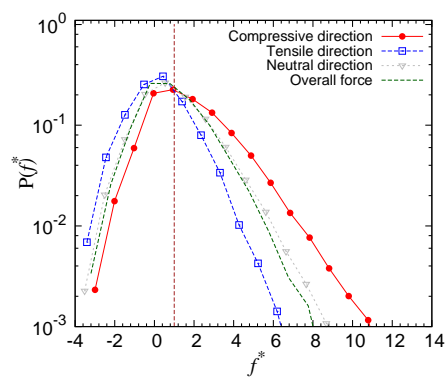

(b)

Figure 5.17: (Color online) Probability distributions of normalized forces $f^{*}=f /\langle f\rangle$ in compressive, tensile, and neutral directions inside the shear bands. Here, we show the results for high pressure in (a) non-cohesive and (b) high cohesive systems. The PDFs of overall normalized forces are also shown.

Figure 5.18 shows the variations of the PDFs along compressive and tensile directions for different values of $B o$. If $B o<1$, the PDFs collapse on top of each other. However, the PDFs get wider with increasing cohesion above $B o=1$ (such widening is more prominent for positive and negative forces in the compressive and tensile directions, respectively). Again, we confirm that strong cohesion leads to an increases of positive and negative forces in the compressive and tensile directions, respectively. Therefore, the force distributions in the principal directions gets more heterogeneous with increasing cohesion for $B o>1$, and hence the heterogeneity of the overall force structure increases.

The results in this section, suggest that for low Bo, compressive forces and shear dominates and governs the distribution of forces along compressive and tensile directions. The forces respond to external compression and shear, i.e., due to shear, particles can rearrange and avoid very large forces. In contrast, for high $\mathrm{Bo}$, cohesion dominates over external compression and the contact forces respond mainly to cohesion and shear. Due to the sticky nature of cohesive forces, rearrangements of the contact network become difficult, and very large contact forces as well as strong sticking forces occur together, and hence the contact network becomes more heterogeneous.

\subsection{Discussion and conclusion}

In this chapter, we have studied the effect of cohesion on shear banding in dry cohesive powders. We used a dimensionless parameter the global Bond number Bo to quantify how strong cohesive forces are relative to compressive forces. We found that $B o \simeq 1$, very well predicts the transition from a free-flowing, non-cohesive system to a cohesive system. Interestingly, we found that many quantities of the system show a transition at $B o \approx 1$. 


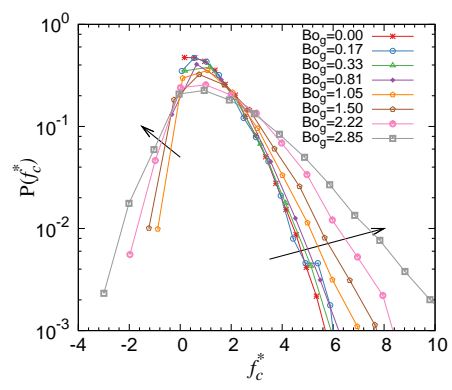

(a)

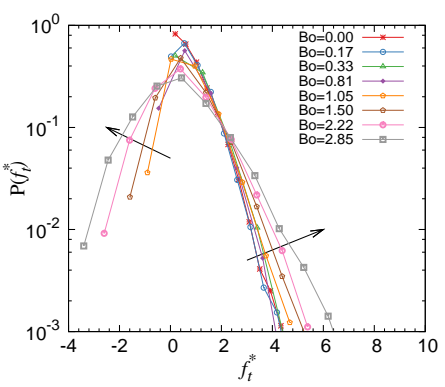

(b)

Figure 5.18: (Color online) Probability distributions of normalized forces in (a) compressive $\left(f_{c}^{*}=f_{c} /\langle f\rangle\right)$ and (b) tensile $\left(f_{t}^{*}=f_{t} /\langle f\rangle\right)$ directions inside the shear bands. Here, we show the results for high pressure and different strength of cohesion, where different symbols represent the global Bond number $B o$ (as given in the inset).

5.4.0.0.1 Shear band Width and center position of the shear band for $B o<1$ stay fairly the same as for non-cohesive material, and show a dependence on cohesion only for $B o \geq 1$. Cohesive forces tend to keep the particles in contact to be connected, i.e. the cohesive forces assist the "collective motion" of particles. As a result, the shear band i.e. the velocity gradient, tends to be flattened. Therefore, the width of the shear band increases with the strength of cohesion, i.e. the Bond number. This would imply that, presence of attractive forces works against the localization of shear.

5.4.0.0.2 Forces and their direction dependence The mean force $\langle f\rangle(P)$ (with $P \propto H-$ $z$ ) is found to be independent of cohesion, like the number of contacts. With increasing $B o$, stronger attractive negative forces are possible at the contact (which is intuitive). However, these negative forces must be balanced by some positive forces to maintain the same overall mean force. Therefore, the positive forces also must become larger as compared to noncohesive systems.

Because we apply shear, compressive/tensile contacts are induced in the system in compressive/tensile eigen-directions of the local strain rate tensor. However, there exists a direction along which no shear takes place. We observe that the mean force along this direction remains unaffected by cohesion, which implies that cohesive forces in the system are induced by shear. Both negative and positive forces are influenced along both tensile and compressive directions.

The mean force carried by contacts along compressive and tensile directions is symmetric about mean overall force. For $B o \leq 1$, this difference i.e. anisotropy of the force network is independent of cohesion, while for $B o>1$ the anisotropy in the force network increases with cohesion. Macroscopically, this anisotropy in force is directly related to the shear stress, we 
observe that the trend in force anisotropy is very similar to the trends found in the shear stress in previous work [107].

5.4.0.0.3 Force probability distribution Since granular systems are known to be heterogeneous in nature, we also analyzed the effect of cohesion on the force probability distributions. For non cohesive systems, no prominent effect of pressure on force distributions could be seen. For high cohesion $B o>1$, pressure affected the distribution of forces, by making the tails longer as compared to the case for $B o \leq 1$. The distribution of forces showed that cohesion makes the force distribution wide, and more symmetric. Splitting up the force distributions along the compressive and tensile directions revealed that, for $B o \leq 1$, the distributions are almost independent of cohesion. For higher $B o$, cohesion broadens the force distributions along tensile direction, which in turn affects the distribution along the compressive direction. This suggests, an increase in heterogeneity in forces for $B o>1$ along compressive and tensile directions. For low $B o$, the dynamics helps the particles to rearrange and avoid very strong forces. In contrast, for high $B o$, cohesion induces stickiness at the contacts so that rearrangements are suppressed, increasing heterogeneity of the system, which is evident from longer tails of the probability distribution for system with higher $B o$.

In conclusion, we have reported that both the flow profiles properties of the system (shear banding) and the force structure are unaffected by cohesion for $B o \leq 1$. In contrast, for $B o>1$, cohesion strongly affects the flow, the anisotropy, and the internal force structure. Attractive forces have been found to reduce shear localization for $B o>1$. In the same regime, cohesion also promoted heterogeneity of the forces. These two observations independently are consistent with previous studies with attractive forces, concerning rheology [30] and force structures for static packings [230].

As speculation for a wider view, our results can be interpreted as follows: In the language of statistical mechanics, $B o$ corresponds to a "control parameter" and $B o=1$ to a "critical point". The critical changes in the characteristic force and the fitting exponent show a small pressure dependence, which could be better predicted using a pressure dependent local Bond number. Since the local $B o$ are close to the global $B o$, the system can be classified by the latter. In our case, the macroscopic properties (position and width of shear-bands) and structural signatures (the tails of the PDFs) gradually increase from $B o=1$. This implies, that this increase, behaves like a "second-order transition". Confirming this would need a further detailed study. Also, experiments performed with controlled cohesive strength would be exciting to confirm and validate our results. Finally it would be interesting to reproduce our findings with different contact models, such as capillary bridges or even simpler linear contacts models. 


\section{A Appendix}

In this appendix, we present the dependence of maximum attractive on local pressure in the system.

5.A.0.0.4 Maximum attractive force The extreme loading and unloading branches are reflected by the outer triangle in Fig. 1 in [102]. Starting from a realized maximum overlap during loading, $\delta_{\max }<\delta_{\max }^{p}$, the unloading happens within the triangle, as can be characterized by a branch with stiffness

$$
k_{2}=k_{1}+\left(k_{p}-k_{1}\right) \delta_{\max } / \delta_{\max }^{p}
$$

(as given in [178]). The elastic, reversible force along this branch is given by $k_{2}\left(\delta-\delta_{0}\right)$ $[102,178]$. The intermediate stiffness $k_{2}$ follows from a linear interpolation between $k_{1}$ and $k_{p}$, as explained in $[102,178]$. The corresponding maximal attractive force is $f_{\mathrm{m}}=$ $-k_{c} \delta_{\min }=-k_{c} \frac{\left(k_{2}-k_{1}\right)}{\left(k_{2}+k_{c}\right)} \delta_{\max }$. If we assume that the maximal overlap $\delta_{\max }^{p}$ is realized under a given external (compressive) pressure $p_{\max }$, then we can infer $\frac{p}{p_{\max }}=\frac{\delta_{\max }}{\delta_{\max }^{p}}$, with pressure $p$ being $p=k_{1} \delta_{\max } / A, A$ being a representative area. This leads to realized maximal attractive force being

$$
f_{\mathrm{m}}=-k_{c} \frac{\left(k_{2}-k_{1}\right)}{\left(k_{2}+k_{c}\right)} \frac{p}{p_{\max }} \delta_{\max }^{p}
$$

Using Eq. (5.9) in Eq. (5.10), we get

$$
f_{\mathrm{m}}=-k_{c} \frac{\left(k_{p}-k_{1}\right) \frac{p_{\max }}{k_{1}}\left(\frac{p}{p_{\max }}\right)^{2}}{k_{c}+k_{1}+\left(k_{p}-k_{1}\right) \frac{p}{p_{\max }}} .
$$

This definition can be used to define a local Bond number as $B o_{l}^{a}(P)=f_{\mathrm{m}}(P) /\langle f(P)\rangle$, where mean force at that pressure is as discussed in Sec. 5.2.3. This Bond number would be compared with various other definitions in Sec. 5.2.3. 


\title{
Chapter 6
}

\section{DEM simulations of granular rheology: Effects of gravity and contact stiffness. ${ }^{*}$}

\begin{abstract}
The chapter presents a characterization of the shear failure behavior for an idealized granular material, under different gravity fields and contact stiffness conditions, where the response is conventionally assumed to be independent of both. A series of Discrete Element simulations are performed on a frictional granular assembly in a split-bottom geometry varying over a wide range of particle softness and gravity. We show that these two properties affect the flow behavior in a very similar fashion. In the steady state, the macroscopic friction decreases systematically with an increase in either particle softness or gravity and the ratio between forces due to gravity and contact stiffness is a suitable non-dimensional parameter to describe the bulk behavior of the material. This trend is traced back to the anisotropy in the contact network, leading to a linear relation between macroscopic friction and deviatoric fabric in the steady state. Interestingly, when the rotation rate of the system is increased the same relation holds, stating that the two properties are related not only in the quasi-static but also in the dense inertial regime.
\end{abstract}

*. Submitted. 


\subsection{Introduction}

Matter is usually classified into solids, liquids, and gases. Granular matter like sand or coffee beans have special properties and do not meet this classification. When poured into a container, it adapts to the shape of the container displaying a property of liquids, at rest it appears solid. While a single grain is clearly solid, granular assemblies have quite different properties. Understanding and modelling these common observations are difficult tasks, so that granular flows have been subject of interest for decades $[45,55,72]$. The flowing behavior of granular materials is important due to its application in geophysics for description and prediction of natural hazards such as landslides, avalanches etc. [55].

Most common granular flows, such as hopper, chute flows and landslides are dense, and can be termed quasi-static flows. There are many ways in which slow granular flows are different from Newtonian flows [226]. For example in slow granular flows the particles have enduring contacts, and inter-particle frictional force is the main source of dissipation. For this kind of flows the relative motion is confined to narrow regions (between large solid-like parts) called shear bands $[22,70,167]$. The flowing regime can be characterized by means of the effective friction coefficient, which is the ratio of shear to compressive stress, that depends on particle and contact properties $[12,190]$. In the case of slow flows, this ratio is known to be independent of local strain rate $[28,55,118,165]$.

In most of the granular flows, like geophysical situation or industrial application, gravity is the main driving force for the flow. Still the effect of changing gravitational acceleration on slow granular flows is largely unexplored. Moreover, this may be particularly important in understanding of the geology on other planets [69]. A poor understanding of failure behavior of soil found on planetary bodies can lead to problems like that of a Mars exploration vehicle getting stuck in granular material [225].

The current understanding of gravitational granular flows, is based on the studies performed under Earth's gravity. Only a couple of studies have been performed on the effect of gravity on granular flows. Klein and White examined the dynamic flow in a tumbler under low gravity on a parabolic flight [85]. They found that the dynamic angle of repose of the flowing layer decreases as $g$ increases. Brucks et al. [26] performed centrifuge experiments at gravity levels larger than Earth's gravity and confirmed that the dynamic angle of repose decreases with increase in $g$. Alshibli et al. [2] investigated the effect of loading condition and confining pressure on the peak friction angle in a conventional triaxial compression test in microgravity and found that peak friction angle in low gravity is higher compared to that found on the Earth consistent with the others. Recent microgravity experiments by Murdoch et al. $[126,127]$ in a Taylor-Couette setup confirm that shear bands can form in the presence of weak gravity fields, just as on earth.

Motivated by these findings, in this paper, we numerically investigate the dependence of the 
bulk behavior of slow granular flows on the gravitational level. Using three-dimensional Discrete Element Method (DEM) simulations, we simulate cohesionless frictional granular material in a split-bottom ring shear cell, as introduced in Ref. [54] and vary gravity by two orders of magnitude. Our simulations explore a range of values, which covers both gravity smaller and larger than Earth's to answer the question, whether or not a unique law could describe the flow behavior on Earth, Moon and Mars. This also enables us to understand the flow properties of granular materials in a broad range of confining pressures, ranging from very low to very high stress levels. Additionally, we study the effect of the normal stiffness of the particles on the bulk behavior, where normal stiffness provides a particle-level effect, while gravity is a macroscopic modification. We find that they have opposite effect at mesoscopic (local) scale, and the macroscopic material behavior can be well described when the ratio between forces due to gravity and contact stiffness is used as a scaling parameter.

We organize this paper as follow: We explain our methods in Sec. 6.2 and show our results for quasistatic state and dense inertial regime in Secs. 6.3 and 6.4, respectively. In Sec. 6.5, we discuss and conclude our results.

\subsection{Discrete Element Method}

In this section, we present our numerical simulations (Sec. 6.2.1) and setup (Sec. 6.2.2). We also summarize various time scales associated with the system (Sec. 6.2.5).

\subsubsection{Model}

We use DEM simulations of soft frictional particles in three-dimension. The normal force between the particles in contact is given by $f_{n}=-k_{n} \delta_{n}-\eta_{n} v_{n}$, where $k_{n}, \delta_{n}, \eta_{n}$, and $v_{n}$ are the normal stiffness, particle overlap, normal viscosity coefficient, and relative velocity in normal direction, respectively. Similarly, the tangential force is introduced as $f_{t}=-k_{t} \delta_{t}-\eta_{t} v_{n}$, where $k_{t}=2 k_{n} / 7, \delta_{t}, \eta_{t}=\eta_{n} / 4$, and $v_{t}$ are the tangential stiffness, relative displacement in tangential direction, tangential viscosity coefficient, and relative velocity in tangential direction, respectively. We also introduce Coulomb's friction between the particles, where the tangential force $f_{t}$ is switched to the sliding force $f_{s}=-\mu_{p}\left|f_{n}\right|$ with the particle friction coefficient $\mu_{p}=0.01$, when $f_{t}$ exceeds the critical value, i.e. $\left|f_{t}\right|>\mu_{p}\left|f_{n}\right|$ [102].

To study the effect of particle softness on macroscopic behaviors, we change the normal stiffness $k_{n}$, as well as the tangential one $k_{t}=2 k_{n} / 7$, within the range $10 \mathrm{~N} / \mathrm{m} \leq k_{n} \leq 10^{4} \mathrm{~N} / \mathrm{m}$. In our simulations, the time increment $\Delta t$ for numerical integrations of the equation of motion is adjusted such that $\Delta t$ is much smaller than the contact duration to ensure accurate dynamic integration [102]. 


\subsubsection{Split-bottom ring shear cell}

We perform numerical simulation in the same setup described earlier in Sec. 2.1.1.1. It consists of two concentric cylinders, with inner and outer cylinder radii as $R_{i}=0.0147 \mathrm{~m}$ and $R_{o}=0.11 \mathrm{~m}$ respectively, with a split radius being $R_{s}=0.085 \mathrm{~m}$ (as shown in Fig. 2.2). The concentric cylinders rotate relative to each other around the symmetry axis (the dotdashed line). The ring shaped split at the bottom separates the moving and static parts of the system, where a part of the bottom and the outer cylinder rotate at the same rate. The system is filled with $N \approx 3.7 \times 10^{4}$ spherical particles with density $\rho=2000 \mathrm{~kg} / \mathrm{m}^{3}=2 \mathrm{~g} / \mathrm{cm}^{3}$ up to height $H$. The cylindrical walls and the bottom are roughened due to some (about $3 \%$ of the total number) attached/glued particles, as explained in [103-105]. When there is a relative motion at the split, a shear band propagates from split position $R_{S}$ upwards and inwards and remains far away from cylinder-walls and bottom in most cases. Since we are interested in the quasi-static regime, the rotation rate of outer cylinder is chosen to be $\frac{\Omega}{2 \pi}=0.01 \mathrm{~s}^{-1}$, such that the inertial number $I \ll 1[118]$.

\subsubsection{Local averaging}

One of the goals of current research in the granular community is to derive macroscopic continuum theory that can take into account the given micro-mechanical properties. Finding a connection between the two scales involves the so-called micro-macro transition [11, 91, 226], often applied on small, seemingly homogeneous representative volume elements (RVE)s [215]. An alternative is to use an inhomogeneous geometry where static and dynamic, flowing zones i.e. high density and dilated zones co-exist - at various confining pressure levels like e.g. in chute flows [226]. In these systems, by local averaging over adequate representative volume elements (RVE)s inside which all particles are assumed to behave similarly, one can obtain local continuum relations covering a wide range of system states. Such a procedure has been developed systematically e.g. in a two-dimensional Couette ring shear cell [91], and a three dimensional split-bottom ring shear cell [103, 107]. The free surface of the split-bottom shear cell allows to scan a range of confining pressures, due to weight of the material, and its maximum is determined by the filling height.

\subsubsection{Averaging and micro-macro procedure}

The averaging procedure used in this study as same as discussed briefly in Sec. 2.3.2.1. From the simulations, we calculate stress and fabric tensors, which are represented as $\sigma_{i j}$ and $F_{i j}$ respectively. It is important to mention that for the rotation rate $\frac{\Omega}{2 \pi}=0.01 \mathrm{~s}^{-1}$, the contribution of the kinetic stress to the total shear stress $\sigma$ is much smaller compared to the contact stress. Hence, we ignore the dynamical stress and concentrate on the contact stress.

For both stress and fabric tensors, we can calculate the eigenvalues and define the volu- 
metric part $T_{\mathrm{v}}=\left(T_{1}+T_{2}+T_{3}\right) / 3$ (pressure $p$ and $F_{\mathrm{V}}$ for stress and fabric respectively) and deviatoric component as $T_{\mathrm{dev}}=\sqrt{\left(\left(T_{1}-T_{2}\right)^{2}+\left(T_{2}-T_{3}\right)^{2}+\left(T_{3}-T_{1}\right)^{2}\right) / 6}\left(\sigma_{\mathrm{dev}}\right.$ and $F_{\mathrm{dev}}$ for stress and fabric respectively). The pressure is the isotropic stress, while $\sigma_{\mathrm{dev}}$ quantifies the normal stress difference. The volumetric fabric $F_{\mathrm{v}}$ represents the contact number density, while the deviatoric fabric $F_{\mathrm{dev}}$ quantifies anisotropy of the contact network.

\subsubsection{Time Scales}

We characterize the dynamics of the system by different time scales focusing on various quantities. At first, we define two microscopic time scales as

$$
T_{c}=2 \pi \sqrt{\frac{\langle m\rangle}{k_{n}}}, \quad T_{\eta}=\frac{\langle m\rangle}{\eta_{n}},
$$

related to the contact duration and the viscous damping between two particles in contact, respectively, where $\langle m\rangle$ is the mass of a particle with mean diameter $\langle d\rangle$. Next, two time scales associated with external forces, i.e. the gravity and external rotation rate, can be introduced as

$$
T_{g}=\sqrt{\frac{\langle d\rangle}{g}}, \quad T_{\Omega}=\frac{2 \pi}{\Omega},
$$

respectively, where $T_{g}$ is the time taken by a particle with zero initial velocity to fall a distance $\langle d\rangle / 2$.

The time scales, Eqs. (6.1) and (6.2), are given by the material constants and applied external forces, respectively, so that these are constants throughout the system. In this sense, the time scales, $T_{c}, T_{\eta}, T_{g}$, and $T_{\Omega}$, are global. On the other hand, we can introduce two macroscopic time scales related to the local shear rate $\dot{\gamma}$ and pressure $p$ as

$$
T_{\dot{\gamma}}=\frac{1}{\dot{\gamma}}, \quad T_{p}=\langle d\rangle \sqrt{\frac{\rho}{p}},
$$

where $T_{p}$ represents the time pressure would take to push a particle away by a distance $\langle d\rangle / 2$. As shown in the following subsections, the spatial distributions of pressure and shear rate are inhomogeneous due to gravity and shear band/localization. Therefore, in contrast to the global time scales, $T_{\dot{\gamma}}$ and $T_{p}$ are local field variables with certain dependence on space.

The time scales can be combined in some dimensionless numbers that give indications of dominance of one of the time scales. For example, the inertial number, $I \equiv T_{p} / T_{\dot{\gamma}}=$ $\dot{\gamma}\langle d\rangle \sqrt{p / \rho}$, which is widely used in previous studies $[35,55,80]$, provides an estimate of the local rapidity of the flow. For $I \ll 1$, the flow is quasistatic, where particles interact via enduring contacts and inertial effects are negligible. For $I \sim 1$, the flow is in the dense inertial regime, and for $I \gg 1$, the flow is in the rapid, collisional gas like state. 
Table 6.1: Table showing $g$ (in units of $\mathrm{ms}^{-2}$ ), particle stiffness $k_{n}$ (in units of $\mathrm{Nm}^{-1}$ ), and various time scales associated with the system, as discussed in the main text (in units of s). The values of these time scales are the average values reported at $z=2\langle d\rangle, H / 2, H-2\langle d\rangle$ in the center of the shear band.

\begin{tabular}{lllllllll}
\hline$g$ & $\Omega$ & $k_{n} \times 10^{2}$ & $T_{c}$ & $T_{g}$ & $T_{\dot{\gamma}}$ & $T_{p}$ & $I \times 10^{-3}$ & $\kappa$ \\
\hline 0.5 & 0.005 & 1 & 0.002 & 0.0663 & $25,20,10$ & $0.017,0.025,0.05$ & $0.7,1.2,5$ & 0.00002 \\
\hline 1 & 0.01 & 1 & 0.002 & 0.0469 & $10.87,7.8,2.73$ & $0.032,0.0153,0.0125$ & $1.3,2,4$ & 0.0005 \\
\hline 2 & 0.01 & 1 & 0.002 & 0.0332 & $10.67,7.47,2.67$ & $0.009,0.011,0.022$ & $0.75,2,1.4$ & 0.0034 \\
\hline 5 & 0.01 & 1 & 0.002 & 0.0210 & $10.28,7.37,2.60$ & $0.005,0.007,0.014$ & $2,0.8,0.9$ & 0.001 \\
5 & 0.01 & 5 & 0.0009 & 0.0210 & $10.61,7.55,2.10$ & $0.005,0.008,0.014$ & $2.5,0.8,1.3$ & 0.00005 \\
\hline 20 & 0.01 & 1 & 0.002 & 0.0105 & $9.67,7.03,2.58$ & $0.0029,0.004,0.008$ & $1,0.5,0.9$ & 0.001 \\
20 & 0.01 & 4 & 0.010 & 0.0105 & $10,7.12,2.66$ & $0.0029,0.004,0.008$ & $0.2,0.4,0.7$ & 0.0001 \\
\hline 50 & 0.01 & 1 & 0.002 & 0.0066 & $8.67,6.64,2.5$ & $0.0074,0.0027,0.002$ & $80,34,72$ & 0.0025 \\
50 & 0.01 & 10 & 0.0006 & 0.0066 & $10.06,7.12,2.6$ & $0.0019,0.0022,0.0046$ & $50,30,60$ & 0.00024 \\
\hline 10 & 0.01 & 1 & 0.002 & 0.0148 & $9.98,7.02,2.61$ & $0.004,0.006,0.01$ & $1.1,0.6,1.5$ & 0.0004 \\
10 & 0.01 & 10 & 0.0006 & 0.0148 & $9.09,8.17,2.63$ & $0.004,0.006,0.01$ & $1.4,0.6,1.1$ & 0.00005 \\
10 & 0.01 & 100 & 0.0002 & 0.0148 & $10.71,7.28,2.79$ & $0.004,0.005,0.011$ & $1.2,0.6,0.9$ & 0.000005 \\
\hline 10 & 0.1 & 1 & 0.002 & 0.0148 & $1.12,0.7,0.23$ & $0.004,0.006,0.009$ & $15,6,9$ & 0.0004 \\
10 & 0.5 & 1 & 0.002 & 0.0148 & $0.25,0.15,0.05$ & $0.004,0.005,0.01$ & $75,26,60$ & 0.0004 \\
10 & 1.0 & 1 & 0.002 & 0.0148 & $0.12,0.07,0.02$ & $0.004,0.005,0.02$ & $150,50,1020$ & 0.0004 \\
10 & 2.0 & 1 & 0.002 & 0.0148 & $0.0210 .03,0.008$ & $0.004,0.006,0.018$ & $300,160,1500$ & 0.0004 \\
\hline
\end{tabular}

Table 6.1 shows typical values of various timescales for our simulations with different normal stiffness and gravity. For slow flows with a rotation rate $\Omega / 2 \pi=0.01 \mathrm{~s}^{-1}$ and the gravity $g \geq 1 \mathrm{~ms}^{-2}$, the inertial number is well below 1 . The inertial number is in the same range, if we use the lower gravity and rotation rate, $g=0.5 \mathrm{~ms}^{-2}$ and $\Omega / 2 \pi=0.005 s^{-1}$, respectively. As the rotation rate increases, the inertial number becomes comparable to 1 .

\subsection{Quasistatic state}

In this section, we present our results on the analysis of macroscopic rheology in a quasistatic state. We will extend our analysis to dense inertial flows in Sec. 6.4. At first, we study the local stress, rheology and the macroscopic friction coefficient in Secs. 6.3.1, 6.3.2 and 6.3.3, respectively. We also show the results of local volume fraction in Sec. 6.3.4, and connect the rheology to the microscopic structure tensor in Sec. 6.3.5.

\subsubsection{Local stress and shear bands}

Figure 6.1 shows the local shear stress, $\tau(r, h)$, plotted against the local pressure, $p(r, h)$, at different positions, i.e. with different local shear rates, $\dot{\gamma}(r, h)$. We observe that for a given pressure, $\tau$ is higher for larger $\dot{\gamma}$, however for $\dot{\gamma}>\dot{\gamma}_{c}$ (with $\dot{\gamma}_{c} \approx 0.08 \mathrm{~s}^{-1}$ ), $\tau$ becomes almost independent of the local strain rate. This means that the slope of the shear stress-pressure 


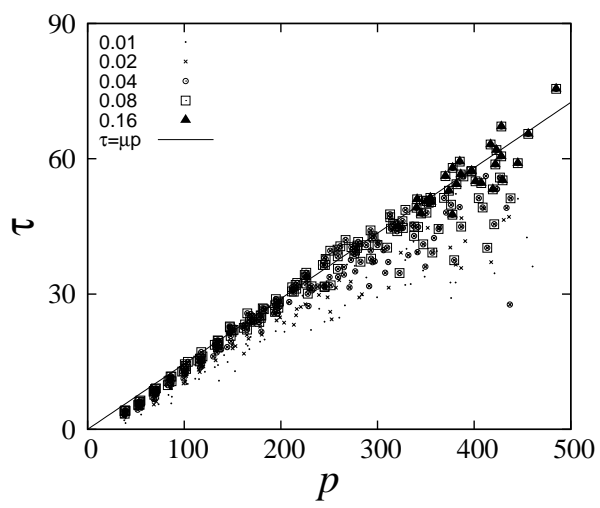

Figure 6.1: (Color online) The local shear stress, $\tau(r, h)$, plotted against the local pressure, $p(r, h)$, for different values of the local shear rate, $\dot{\gamma}(r, h)$ as given in the inset, under the gravity, $g=10 \mathrm{~ms}^{-2}$.

curve is almost constant for all data points with strain rate larger than the threshold value. In other words, if the dimensionless shear length $l_{\gamma}=t_{a v} \dot{\gamma}$ [103] exceeds unity, i.e. neighboring particles are sheared about one particle diameter, the shear deformation can be assumed to be fully established, which is a concept of the critical flow regime [103]. Ries et al. [159] showed that a minimum strain is required to reach the critical state, which is a unique state regardless of the preparation history of the material [227]. MRI experiments from Sakaie et al. [164] also showed that the location where the strain is above a cutoff coincides with the dilatancy zone, which on the other hand coincides with the region inside the shear band. Our previous works $[103,107]$ showed, that for rotation rate $\Omega / 2 \pi=0.01 \mathrm{~s}^{-1}, \dot{\gamma}_{c} \approx 0.08 \mathrm{~s}^{-1}$ is the shear-rate above which the shear-bands are well established. Since we are interested in the flowing behavior in the steady state, in the rest of the paper, we analyze only the data in shear bands,

$$
\dot{\gamma}(r, h)>\dot{\gamma}_{c}(\Omega) \equiv \frac{4 \Omega}{\pi} .
$$

\subsubsection{Rheology}

To understand the rheology and dilatancy in the system, we analyze the relations between the local shear stress and shear rate, and the local pressure and shear rate, respectively. Figure 6.2 displays the local shear stress, $\tau(r, h)$, and pressure, $p(r, h)$, plotted against the local shear rates above the cutoff shear rate, $\dot{\gamma}(r, h)>\dot{\gamma}_{c}(\Omega)$ for $\Omega / 2 \pi=0.01 \mathrm{~s}^{-1}$. Here, the local shear rates are restricted to relatively small values (due to small rate of rotation) and we find that both the shear stress and pressure are fairly constant against the shear rate. Such states with small shear rates can be assumed to be in quasistatic state, where both the shear stress and pressure are almost independent of the strain rate in accordance with Refs. [44, 166, 192]. 

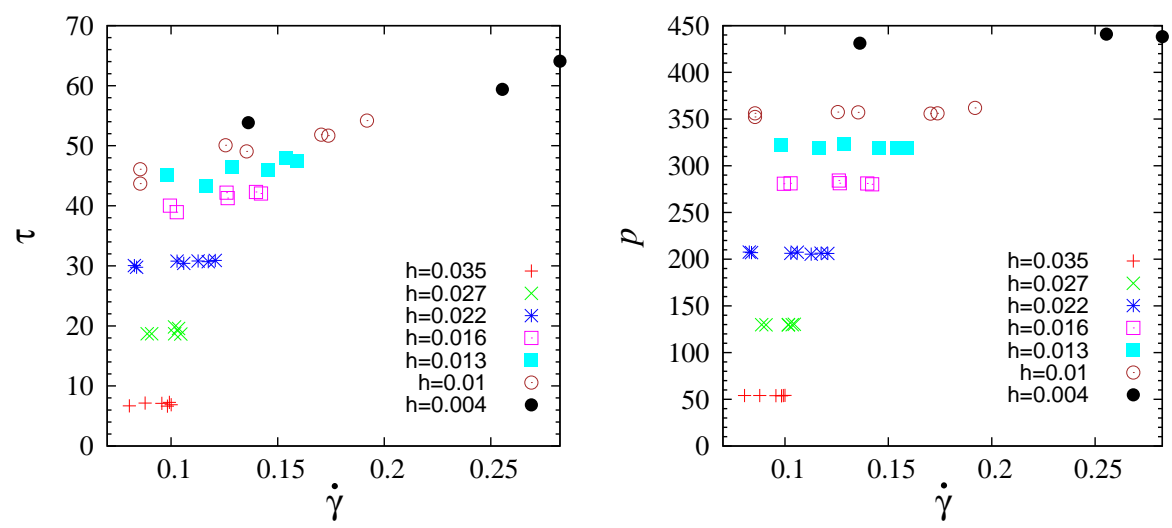

Figure 6.2: (Color online) The (Left) local shear stress, $\tau(r, h)$, and (Right) pressure, $p(r, h)$, plotted against the local shear rate, $\dot{\gamma}(r, h)$. Different symbols show different height $h$ in the system as given in the inset.

Note that both the shear stress and pressure increase as the height decreases, which is due to the weight of the material above a layer at height $h$. Fig. 6.3 displays the local pressure against the height, where the pressure is well approximated by

$$
p(r, h) \simeq \rho g(H-h)
$$

\subsubsection{Friction coefficient}

In a quasistatic state, both the local shear stress and pressure do not much depend on the local shear rate, which means that the local friction coefficient, or shear stress ratio defined as

$$
\mu(r, h) \equiv \frac{\tau(r, h)}{p(r, h)}
$$

also does not depend on the local strain rate, a property of the quasistatic state. In previous studies, the friction coefficient has been assumed to be independent of both the particle stiffness and gravity. However, the particles used in many previous studies were extremely rigid and there are few works systematically investigating the dependence of the flow behavior on gravity. Thus, we study the dependence of the local friction coefficient on the particle stiffness and gravity, restricting ourselves to quasistatic states so that we can neglect the weak dependence on the local strain rate.

Figure 6.4a displays the shear stress-pressure curve for different values of normal stiffness, $k_{n}$, where the softness of the particles drastically decreases the shear stress. Figure $6.4 \mathrm{~b}$ displays the shear stress-pressure curve for different values of gravity, $g$, where the shear 


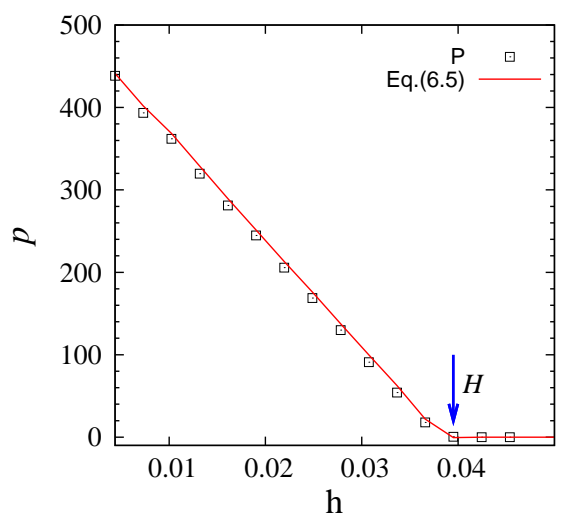

Figure 6.3: (Color online) The local pressure, $p(r, h)$, plotted against the height $h$ in the system, under the gravity, $g=10 \mathrm{~ms}^{-2}$. The line is the approximation given by Eq. 6.5.

stress under higher gravity is always smaller than that under lower gravity. From these results, the local friction coefficient in a quasistatic state, $\mu_{0}$, certainly depends on both softness and gravity.

\subsubsection{Linear approximation}

To understand the dependence of the macroscopic friction coefficient in a quasistatic state on the softness and gravity, we estimate it as the slope of a linear fitting function for the shear stress against pressure, i.e.

$$
\tau(r, h) \simeq \mu_{0}^{\text {global }} p(r, h),
$$

where $\mu_{0}^{\text {global }}$ is a global friction coefficient which depends neither on the shear rate nor on pressure.

Figure 6.5a displays the global friction coefficient $\mu$ plotted against gravity $g$ for different values of the normal stiffness, $k_{n}$, as given in the inset. We observe that $\mu$ decreases with increasing gravity, while it increases with increasing $k_{n}$. Figure $6.5 \mathrm{~b}$ shows the global friction coefficient with different values of the normal stiffness, $k_{n}$, and gravity, $g$, where all results of $\mu_{0}^{\text {global }}$ are collapsed if we introduce the global compressibility,

$$
\kappa \equiv\left(\frac{T_{c}}{T_{g}}\right)^{2}=\frac{\langle m\rangle g}{k_{n}\langle d\rangle},
$$

defined as the square of the ratio between the two time scales $T_{c}$ and $T_{g}$. The global compressibility, $\kappa$, provides a global measure of compressibility of the bulk material. A high $\kappa$ signifies that the bulk material is compressible, which comes from very high confinement by the gravity or low contact stiffness at particle level. On the other hand, when $\kappa$ is small, the 

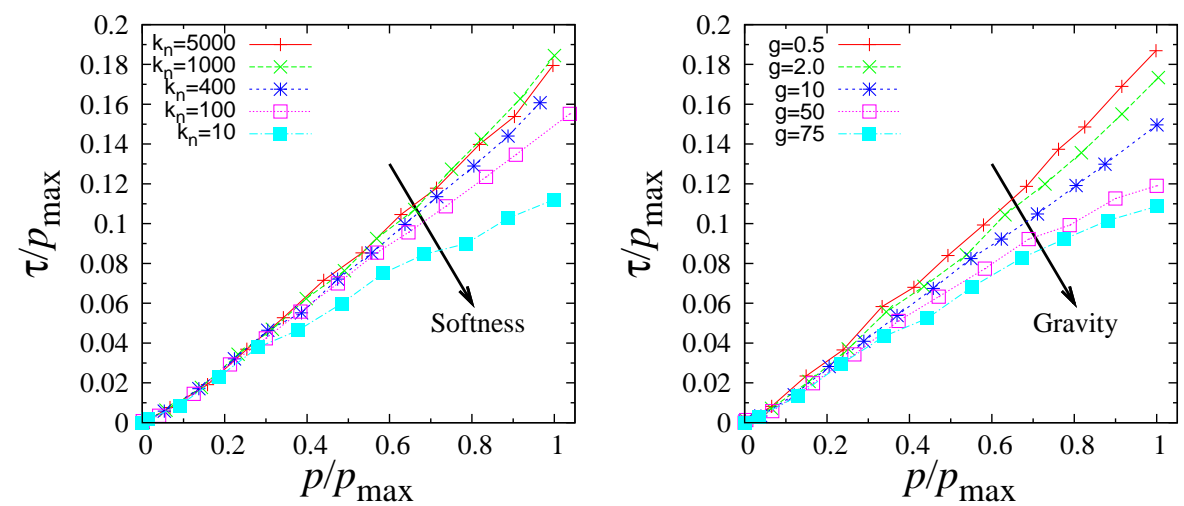

Figure 6.4: (Color online) (Left) The local shear stress plotted against the local pressure for different values of the normal stiffness as given in the inset in units of $\mathrm{kg} / \mathrm{s}^{2}$. Here, the gravity is fixed to $g=10 \mathrm{~m} / \mathrm{s}^{2}$. (Right) The local shear stress plotted against the local pressure for different values of the gravity as given in the inset in units of $\mathrm{m} / \mathrm{s}^{2}$. Here, the normal stiffness is fixed to $k_{n}=10 \mathrm{~m} / \mathrm{s}^{2}$. Both $\tau(r, h)$ and $p(r, h)$ are scaled by the maximum pressure $p_{\max }(r, h)$, respectively. Both $\tau(r, h)$ and $p(r, h)$ are plotted in the center of the shear band.

average overlap is very small compared to the particle diameter, which means that the bulk material is closer to being the rigid limit.

In Fig. 6.5b, the solid line is given by

$$
\mu_{0}^{\text {global }}=\mu_{r}^{\text {global }}-\left(\frac{\kappa}{\kappa_{0}}\right)^{\alpha},
$$

where $\mu_{r}^{\text {global }}$ is the global friction coefficient in the rigid particle limit, and the exponent and characteristic global compressibility are given by $\alpha \simeq 0.4 \pm 0.01$ and $\kappa_{0} \simeq 3.55$, respectively.

Note that Klein and White [85] and Brucks et al. [26] showed similar dependence of the macroscopic friction coefficient on gravity, where they found this dependence might be coming from cohesive forces in micro-gravity and/or a load-dependent interparticle friction coefficient. However, they focused on the dynamic flows, while our results are for slow granular flows and no cohesive force or load-dependent friction was implemented in any of the DEM simulation data presented here.

\subsubsection{Nonlinearity}

In the shear stress-pressure curves for different softness and gravity (Fig. 6.4), the dependence of shear stress on pressure slightly "bends" with increasing softness and gravity. This 

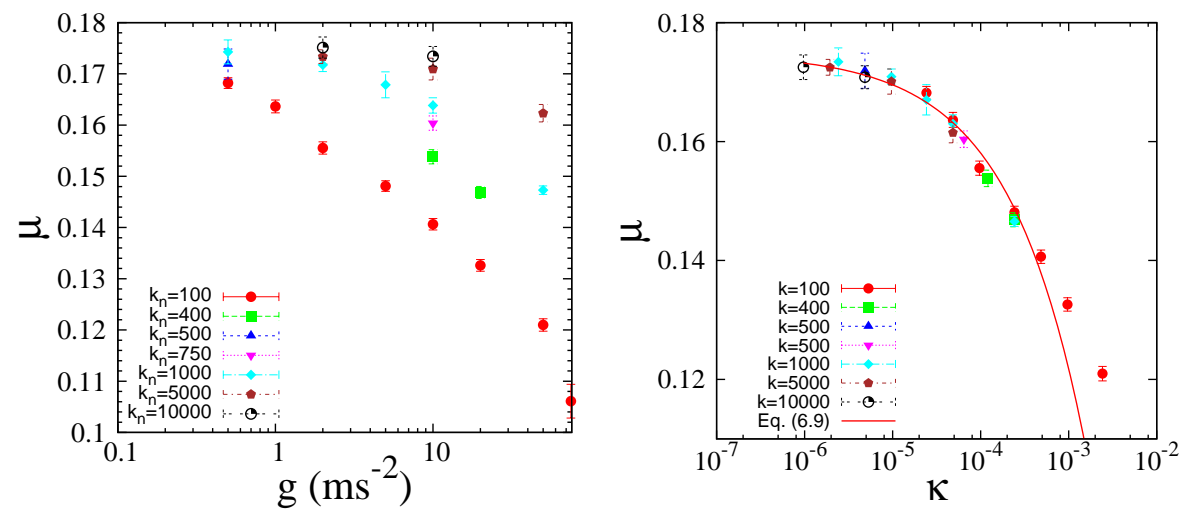

Figure 6.5: (Color online) The global friction coefficient, $\mu_{0}^{\text {global }}$, plotted against (Top) gravity $g$, and (Bottom) the global compressibility, $\kappa=m g /\left(k_{n}\langle d\rangle\right)$, on a log-linear scale for different values of the normal stiffness and gravity as shown in the inset. The solid line represent the corresponding fit to Eq. (6.9).

means that the friction coefficient depends on the pressure and the shear stress becomes a nonlinear function of pressure, i.e.

$$
\tau(r, h)=\mu_{0}^{\text {local }}(p) p(r, h)
$$

where $\mu_{0}^{\text {local }}(p)$ is a local friction coefficient which depends on pressure, but not on the shear rate, since we excluded large strain-rate data.

Figure 6.6 shows the local friction coefficient with different values of the normal stiffness and gravity, where all results of $\mu_{0}^{\text {local }}(p)$ are well collapsed if we introduce the local compressibility,

$$
p^{*} \equiv\left(\frac{T_{c}}{T_{p}}\right)^{2}=\frac{p\langle d\rangle}{k_{n}},
$$

defined as the ratio between two time scales, $T_{c}$ and $T_{p}$. Therefore, we rewrite the local friction coefficient as a function of the local compressibility, $\mu_{0}^{\text {local }}\left(p^{*}\right)$. In this figure, we scanned through a wide range of $p^{*}$ by systematically varying $g$ and $k_{n}$, and observe that for $p^{*}<5 \times 10^{-4}, \mu_{0}^{\text {local }}\left(p^{*}\right)$ is almost constant, while for higher values $\mu_{0}^{\text {local }}\left(p^{*}\right)$ decreases with $p^{*}$ up to $p^{*} \approx 0.1$. This dependence can be written in the form,

$$
\mu_{0}^{\text {local }}\left(p^{*}\right)=\mu_{\mathrm{r}}^{\text {local }}-\left(\frac{p^{*}}{p_{\sigma}^{*}}\right)^{\beta_{1}}
$$

where $\mu_{\mathrm{r}}^{\text {local }}=0.172$ is the value of macroscopic friction in the rigid limit, which is in fair agreement with contact dynamics simulations [208]. The exponent is found to be $\beta_{1} \approx$ 


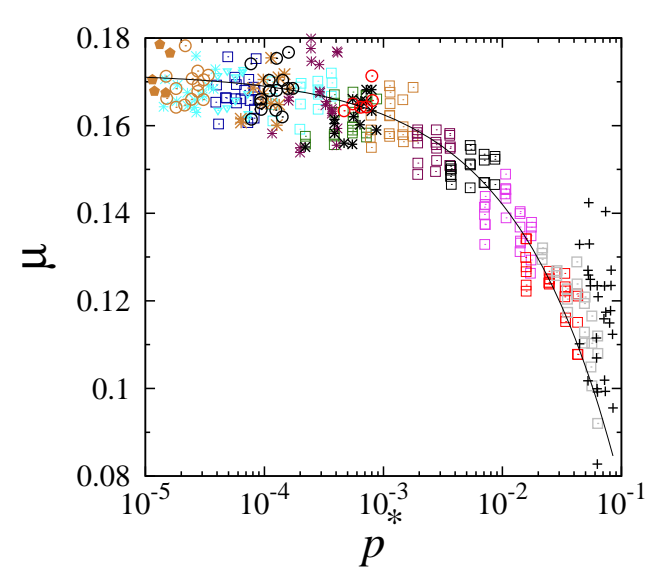

Figure 6.6: (Color online) The local friction coefficient, $\mu_{0}^{\text {local }}\left(p^{*}\right)$, plotted against the local compressibility, $p^{*}$, on a log-linear scale. Different symbols represent different values of $\kappa$ as given in the inset of Fig. 6.7, while the solid line represent the corresponding fit to Eq. (6.12).

$0.5 \pm 0.04$ and the characteristic local compressibility is $p_{1}^{*}=10.08 \pm 0.2$. As one extreme of $p^{*}$, at $p^{*}=0.1$, the average overlap is almost $10 \%$ relative to the mean particle diameter, that is the soft particle limit. The upper bound of $\mu_{0}^{\text {local }}\left(p^{*}\right)$ is the low compression case, where the average overlap is much smaller relative to the particle diameter, and where $\mu_{0}^{\text {local }}\left(p^{*}\right)$ is almost double as large as for $p^{*} \approx 0.1$.

From Eqs. (6.5), (6.8), and (6.11), the global and local compressibilities, $\kappa$ and $p^{*}$, are connected by a relation

$$
p^{*}\left(h^{*}\right)=\kappa\left(H^{*}-h^{*}\right)
$$

or

$$
\kappa=\frac{2}{H^{*}}\left(\frac{1}{H^{*}} \int_{0}^{H^{*}} p^{*} d h^{*}\right),
$$

where we introduced the scaled heights, $h^{*} \equiv h /\langle d\rangle$ and $H^{*} \equiv H /\langle d\rangle$. Therefore, the global compressibility, $\kappa$, is proportional to the height average of the local compressibility, $p^{*}$.

\subsubsection{Local volume fraction}

In Fig. 6.7, the local volume fraction $v$ is plotted against the local compressibility, $p^{*}$, where the packing is rather loose for lower $p^{*}$ and tends to a critical value $v_{c}=0.642$. The data can be very well fitted by

$$
p^{*}=a^{*}\left(v-v_{\mathrm{c}}\right)
$$




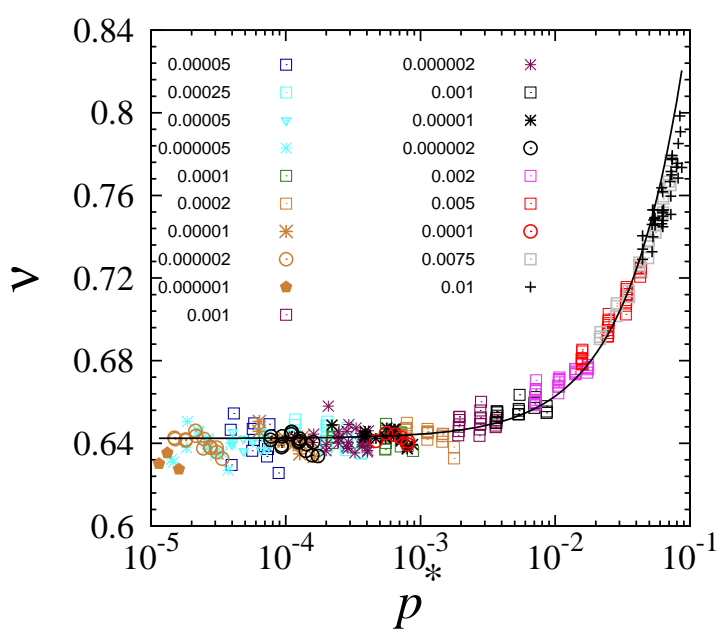

Figure 6.7: (Color online) The local volume fraction, $v(r, h)$, in the system plotted against the local compressibility, $p^{*}$, on a semi-logarithmic scale. Different symbols represent different values of $\kappa$ as given in the inset. The solid line represents the corresponding fit to Eq. (6.15).

with and $a^{*}=0.48$. Interestingly, no significant difference in volume fraction $v$ is observed for $p^{*}<10^{-3}$, while for $p^{*}>10^{-3}$ within the fluctuations, $v$ begins to increase almost linearly with $p^{*}$ (the curvature is due to logarithmic axis). The relation between $v$ and $p^{*}$ is well established in the case of static packings [58, 71, 233]. Here we show that the same relation holds for a slow granular flow, involving considerable finite strain rates.

\subsubsection{Local structures}

The shear resistance in a dense granular flow often accompanies the microscopic anisotropy. Previous experiments have also shown that persistent shear leads to the buildup of a deviatoric fabric in the system $[111,214]$. To relate such a structure to the rheology in a quasistatic state, we analyze the fabric tensor. The second invariant of the fabric tensor quantifies anisotropy of the contact network in the system.

\subsubsection{Anisotropy}

Figure 6.8 displays the local deviatoric fabric, $F_{\mathrm{dev}}(r, h)$, plotted against the local compressibility, $p^{*}$, where $F_{\mathrm{dev}}(r, h)$ for different values of the particle stiffness and gravity is well collapsed on a unique curve (solid line). This dependence can be written in a similar fashion as Eq. (6.12),

$$
F_{\mathrm{dev}}\left(p^{*}\right)=F_{\mathrm{dev}}^{r}-\left(\frac{p^{*}}{p_{F}^{*}}\right)^{\beta_{2}},
$$




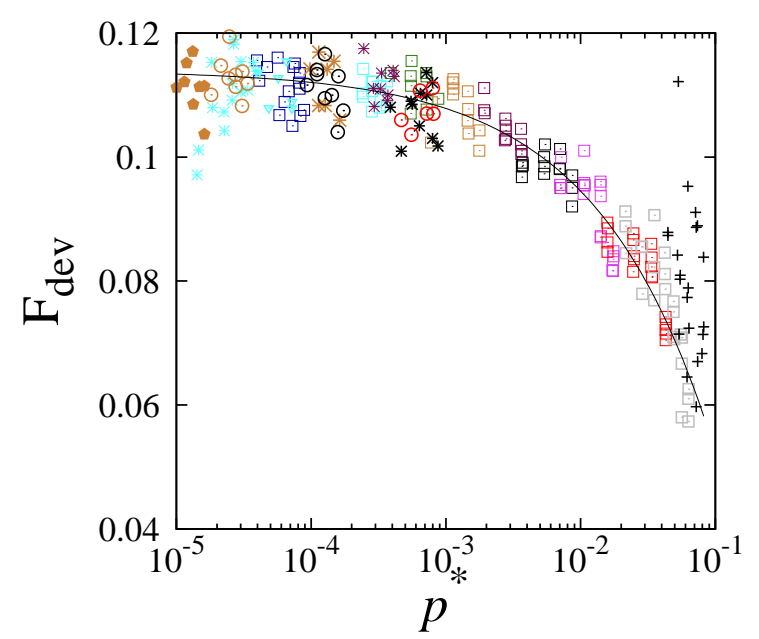

Figure 6.8: (Color online) The local deviatoric fabric, $F_{\mathrm{dev}}(r, h)$, plotted against the local compressibility, $p^{*}$, on a log-linear scale. Different symbols represent different values of $\kappa$ as given in the inset of Fig. 6.7. The solid line represent the corresponding fit to Eq. (6.16).

where $F_{\mathrm{dev}}^{r}$ is the anisotropy of contact network in the rigid limit, the exponent is found to be $\beta_{2} \approx 0.5 \pm 0.03$, and $p_{F}^{*} \approx 26.34 \pm 0.6$.

In Figs. 6.6 and 6.8, we observe that the the local shear resistance and the local anisotropy of the contact network in a quasistatic state show very similar dependence on the local compressibility. In Fig. 6.9, we plot $\mu_{0}\left(p^{*}\right)$ against $F_{\operatorname{dev}}\left(p^{*}\right)$ for different values of $\kappa$, where one can see a linear relation between

$$
\mu_{0}^{\text {local }}\left(p^{*}\right)=\mu_{\text {iso }}+b F_{\mathrm{dev}}\left(p^{*}\right)
$$

where $\mu_{\text {iso }}=0.01 \pm 0.01 \approx 0$ is the friction coefficient in the (extrapolated) limit of an isotropic contact network and $b=1.38$ is a constant of proportionality. The shear resistance accompanies the anisotropy in the contact network. This observation supports the idea presented in recent studies $[87,88,94,236]$, where the authors claim that fabric is an essential ingredient to uniquely characterize the critical state of a granular system.

\subsubsection{Shape factor}

Moreover we compare the shape factor $\left(\frac{T_{2}}{T_{1}}\right)$ for stress and fabric tensors, where $T_{2}$, and $T_{1}$ are the eigenvalues of the deviatoric tensors.

In Fig. 6.10a, we plot the shape factor for the stress tensor. We observe that it is slightly below zero. In Fig. 6.10b, the shape factor for fabric tensor is fluctuates around zero. These two observations suggest that the fabric and stress tensors behave differently even though 


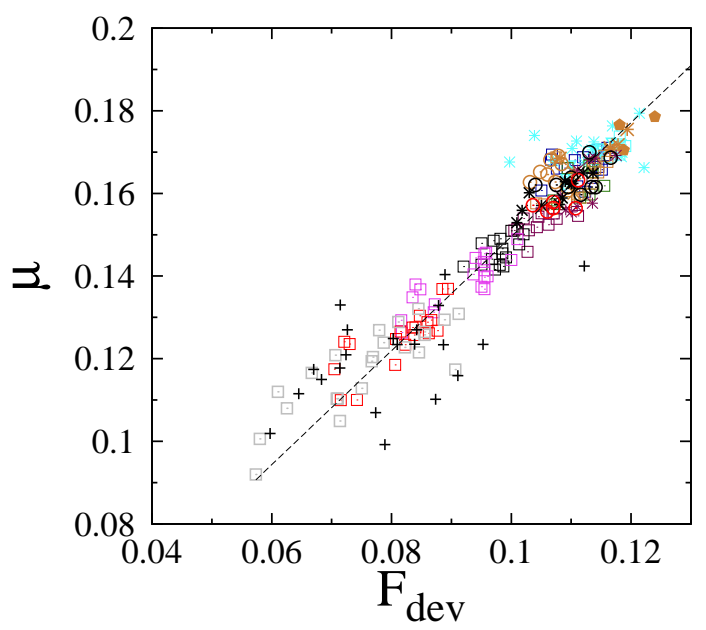

Figure 6.9: (Color online) The local friction coefficient, $\mu_{0}^{\text {local }}\left(p^{*}\right)$, plotted against the local deviatoric fabric, $F_{\mathrm{dev}}(r, h)$, for different values of $\kappa$. Different symbols represent different values of $\kappa$ as given in the inset of Fig. 6.7. The solid line represent the corresponding fit to Eq. (6.17).

they are proportional in magnitude (norm) as shown in Fig. 6.9. The fabric tensor is in a planar state very much like the strain rate, whereas stress is in a partly triaxial state, as expected for a solid-like material. $F_{2} / F_{1}$ tends to positive values of larger $p^{*}$, establishing the difference between structure and stress tensors.

\subsection{Dense inertial regime}

In the previous section, we showed that the friction coefficient and deviatoric fabric are strongly correlated in a quasistatic state. Motivated by this, we check this correlation in the dense inertial regime. In Sec. 6.4.1 we show the dilatancy law in the dense inertial regime. In Secs. 6.4.2, and 6.4.3 we extend our analysis of the friction coefficient and anisotropy to the dense inertial regime. In Sec. 6.4.4, we examine the correlation between the rheology and microscopic structure in the dense inertial regime.

\subsubsection{Dilatancy Law}

Figure 6.11 displays the local volume fraction plotted against inertial number $I$. The quasistatic state data for different gravity and stiffness (from Sec. 6.3) is superimposed with the different external rotation rate data for a given gravity $g=10 \mathrm{~ms}^{-2}$ and stiffness $k_{n}=$ $100 \mathrm{Nm}^{-1}$. Two trends show up: (a) Different gravity and softness data for low $I \approx 0.001$ show an increasing trend, which is mainly due to $1 / \sqrt{P}$ in $I$. However $v$ does not scale 

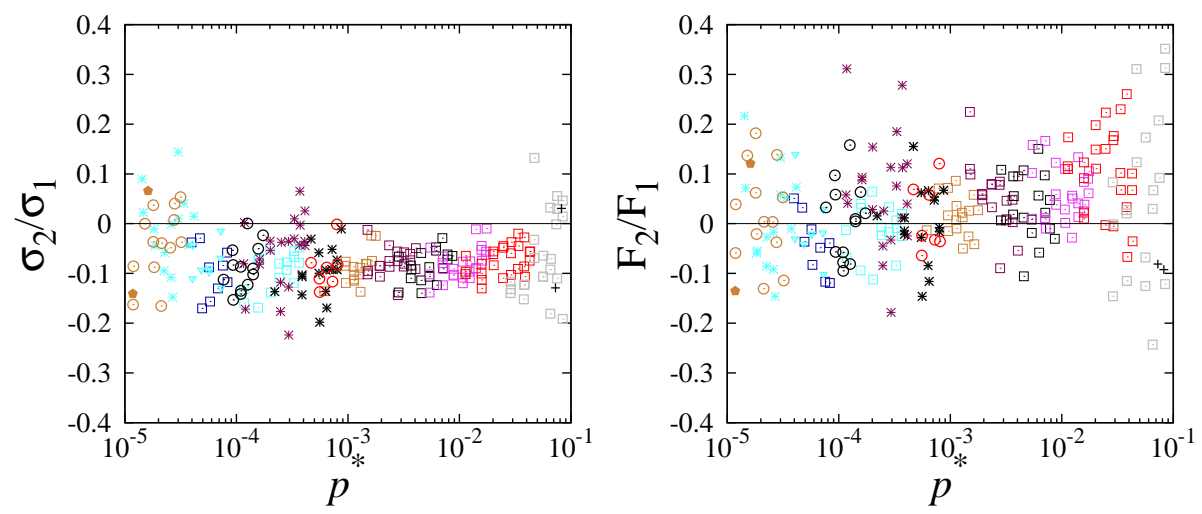

Figure 6.10: (Color online) Shape factor for (a) stress, and (b) fabric plotted against dimensionless pressure $p^{*}$. Different symbols represent different values of $\kappa$ as given in the inset of Fig. 6.7.

with $I$. This data was found to scale with $p^{*}$, as explained previously. (b) $v$ calculated from simulations with different external rates of rotation for gravity $g=10 \mathrm{~ms}^{-2}$ and stiffness $k_{n}=100 \mathrm{Nm}^{-1}$ however show a different trend. We observe that $v$ decreases approximately linearly with increasing $I$. This data very well collapses on the relation:

$$
v\left(I, p^{*}\right)=v_{0}^{\text {local }}\left(p^{*}\right)-b_{v} I
$$

which includes both the compressibility and inertial effect, with $v_{0}^{\text {local }}\left(p^{*}\right)$ as given b y Eq. (6.15) and $b_{v}=0.9 \pm 0.05$. For $I \leq 0.01, v$ stays almost constant, and the system is in almost rate independent quasistatic regime, which was explored in the previous section. However for $I>0.01$, the inertial effects begin to dominate and the system is found to dilates.

\subsubsection{Friction coefficient}

In this section, we analyze the dependence of friction coefficient $\mu$ on inertial number $I$.

Figure 6.12 displays the local friction coefficient plotted against inertial number $I$. The quasistatic state data for different gravity and stiffness (from Sec. 6.3) is superimposed with the different external rotation rate data for a given gravity $g=10 \mathrm{~ms}^{-2}$ and stiffness $k_{n}=$ $100 \mathrm{Nm}^{-1}$. Once again, two trends show up: (a) Different gravity and softness data for low $I \approx 0.001$ does not scale with $I$. This data was found to scale with $p^{*}$, as explained previously. (b) $\mu$ calculated from simulations with different external rates of rotation for gravity $g=10 \mathrm{~ms}^{-2}$ and stiffness $k_{n}=100 \mathrm{Nm}^{-1}$ however show a different trend. We observe that $\mu$ increase approximately linearly with increasing $I$, starting from $\mu_{0}^{\text {local }}\left(p^{*}\right)$. This data very well collapses on the relation:

$$
\mu\left(I, p^{*}\right)=\mu_{0}^{\text {local }}\left(p^{*}\right)+b_{\mu}\left(p^{*}\right) I,
$$




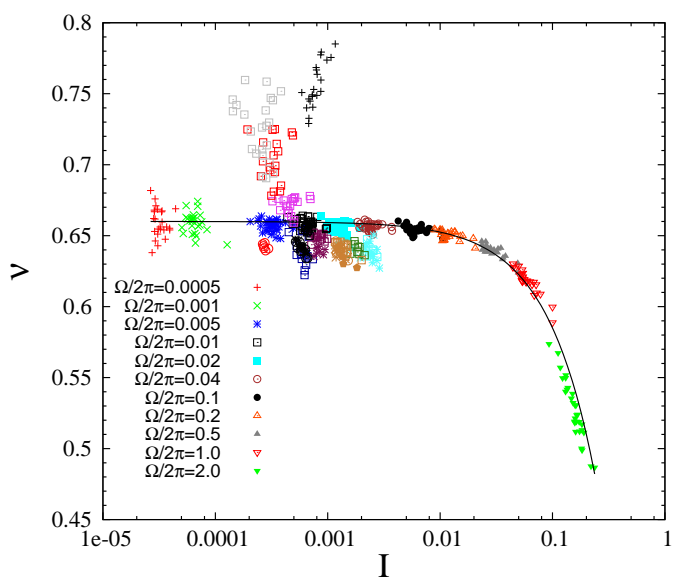

Figure 6.11: (Color online) Local volume fraction plotted against the inertial number $I$ for results from simulations with different rates of rotation. Different symbols represent different rates of rotation as given in the inset and rest symbols are same as given in Fig. 6.7.

which includes both the compressibility and inertial effect, with $\mu_{0}^{\text {local }}\left(p^{*}\right)$ as given b y Eq. (6.12) and $b_{\mu}=1.08 \pm 0.05$. For $I$ for $I \leq 0.01$, the $\mu$ is in rate independent quasistatic state, which means it has almost no dependence on $\dot{\gamma}$, but depends on $p^{*}$. However for for $I>0.01$, the friction coefficient increases linearly with $I$.

\subsubsection{Anisotropy}

Fig. 6.13 displays the local $F_{\text {dev }}$ plotted against inertial number $I$. The quasistatic state data for different gravity and stiffness (from Sec. 6.3) is superimposed with the different external rotation rate data for a given gravity $g=10 \mathrm{~ms}^{-2}$ and stiffness $k_{n}=100 \mathrm{Nm}^{-1}$. Once again, two trends show up: (a) Different gravity and softness data for low $I \approx 0.001$ does not scale with $I$. This data was found to scale with $p^{*}$, as explained previously. (b) $F_{\mathrm{dev}}$ calculated from simulations with different external rates of rotation for gravity $g=10 \mathrm{~ms}^{-2}$ and stiffness $k_{n}=100 \mathrm{Nm}^{-1}$ however show a different trend. We observe that $F_{\mathrm{dev}}$ increase approximately linearly with increasing $I$. This data very well collapses on the relation:

$$
F_{\mathrm{dev}}\left(I, p^{*}\right)=F_{\operatorname{dev}_{0}}^{\text {local }}\left(p^{*}\right)+b_{F_{\mathrm{dev}}}\left(p^{*}\right) I,
$$

with $F_{\text {dev }_{0}}^{\text {local }}$ as given by Eq. (6.16) and $b_{F_{\mathrm{dev}}} \approx 0.9$. It is noticeable that for a given $I$, the scatter in $F_{\mathrm{dev}}$ is more pronounced compared to that in $\mu$. For $I>0.1, F_{\mathrm{dev}}$, shows a very different behavior as predicted by Eq. (6.20), and a decreasing trend is observed. This might be due to the fact that around this $I$, packing becomes very loose, it is noticeable that for $I \approx 0.05$, volume fraction $v \leq 0.55$. It is interesting to note that for $I \leq 0.05$, the anisotropy in contact network increases with increasing $I . F_{\mathrm{dev}}$ varies oppositely with the volume fraction, but varies very much like the friction coefficient. 


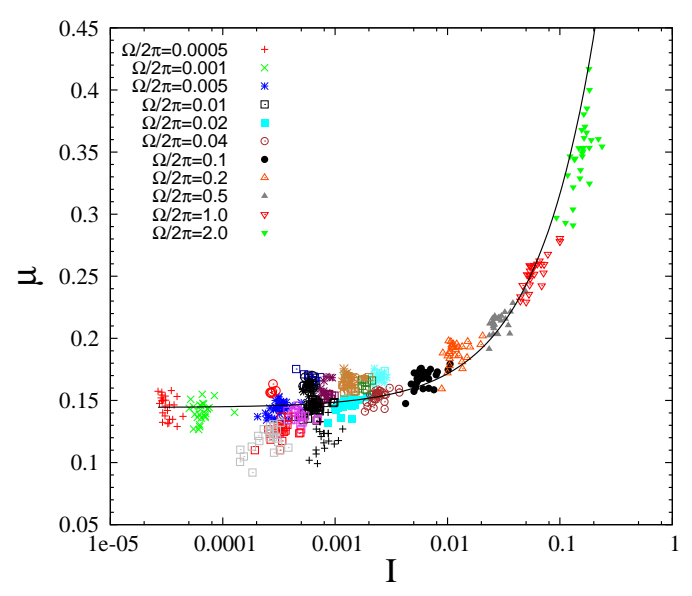

Figure 6.12: (Color online) The friction coefficient plotted against the inertial number $I$ for results from simulations with different rates of rotation. Different symbols represent different rates of rotation as given in the inset and rest symbols are same as given in Fig. 6.7.

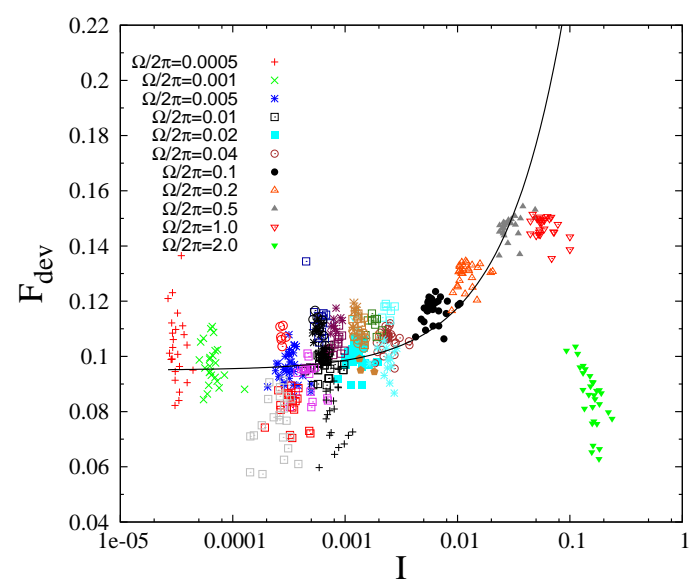

Figure 6.13: (Color online) The local deviatoric fabric $F_{\text {dev }}$ plotted against the inertial number $I$ for results from simulations with different rates of rotations. Different symbols represent different rates of rotation as given in the inset and rest symbols are same as given in Fig. 6.7. 


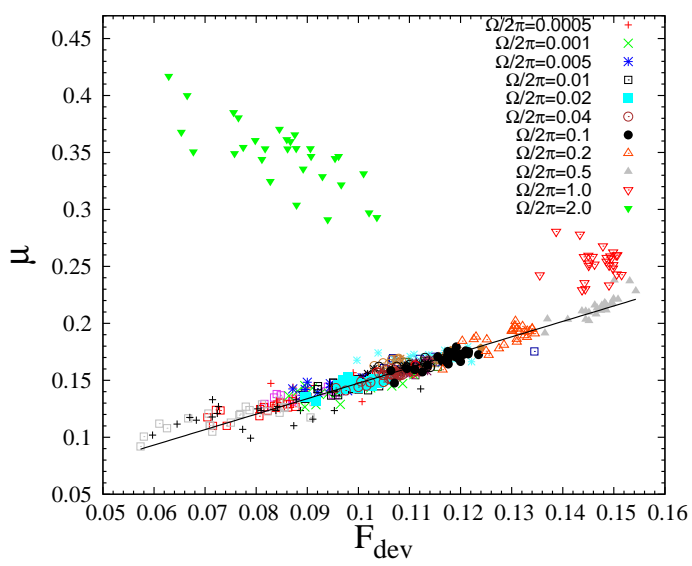

Figure 6.14: (Color online) $\mu$ plotted against $F_{\mathrm{dev}}$ for data in previous section and different rates of rotation for $g=10$. Different symbols represent different rates of rotation as given in the inset and rest symbols are same as given in Fig. 6.7.

\subsubsection{Correlations with structures}

Figure 6.14 shows $\mu$ plotted against $F_{\mathrm{dev}}$ for different rates of rotation. In the same figure, we also plot the quasistatic data with different gravity and stiffness in Sec. 6.3. All the data collapses the linear relation used in Fig. 6.9. Only for very high rate of rotation, $F_{\text {dev }}$ drops off (the packing becomes very loose for such rate of rotation). Interestingly, the same law as proposed in quasi-static regime (where contact stress dominates) hold in the dense inertial regime too. It is important to point out that the data plotted scans over at least three orders of magnitude in both $\kappa$ and $I$, which further adds strength to this result. This suggests that the local contact network dominates the behavior in the high inertial regime also.

\subsection{Discussion and Conclusion}

In this paper, we have studied the rheology of three-dimensional frictional granular assembly.

For slow quasistatic flows, we systematically varied gravity and stiffness to study the effect of them on the flow behavior. Our data shows that the macroscopic friction coefficient $\mu$ decreases with increase in both gravity $g$ and softness. When the same data are plotted against a control-parameter $\kappa$ (which is the ratio of two time scales), the data collapse on a unique curve. Even though, $k_{n}$ and $g$ affect the force between particles at different scales, they have equal and opposite effect at the mesoscale (local scale). Both of them have an effect on the flow behavior by modifying the microstructure, as quantified by the deviatoric fabric. Both deviatoric fabric $F_{\mathrm{dev}}$ and macroscopic friction $\mu$ can be expressed as very 
similar power law functions of the local compressibility $p^{*}$. This proves that they are highly correlated in quasistatic regime and the $\mu$ follows contact anisotropy.

We further studied the rheology of the system for gravity $g=10 \mathrm{~ms}^{-2}$, and we found that for faster flows the system enters into a rate dependent dense, inertial regime. We find that both $\mu$ and $F_{\mathrm{dev}}$ show very similar dependence on $I$, which shows that both are correlated even in the dense inertial regime. This correlation fails only for very fast flows, as the packing becomes too loose at such high shear rates. This shows that the contact network dominates the behavior in slow and moderately fast granular flows too. The fact that for very fast flows, i.e., $I \geq 0.1$ friction coefficient does not follow contact anisotropy deserves a detailed study. 


\section{Bibliography}

[1] M. P. Allen and D. J. Tildesley. Computer Simulations of Liquids. Clarendon Press, Oxford, 1989.

[2] K. A. Alshibli, M. Asce, S. N. Batiste, and F. Asce. Strain Localization in Sand: Plane Strain versus Triaxial Compression. Journal of Geotechnical and Geoenvironmental Engineering, 129(6), 2003.

[3] S. J. Antony. Evolution of force distribution in three-dimensional granular media. Physical Review E, 63(1):011302, 2000.

[4] S. J. Antony. Link between single-particle properties and macroscopic properties in particulate assemblies: role of structures within structures. Philosophical Transactions of the Royal Society A: Mathematical, Physical and Engineering Sciences, 365(1861), 2007.

[5] S. J. Antony and N. P. Kruyt. Role of interparticle friction and particle-scale elasticity in the shear-strength mechanism of three-dimensional granular media. Physical Review E (Statistical, Nonlinear, and Soft Matter Physics), 79:031308(3), 2009.

[6] E. Azéma and F. Radjai. Stress-strain behavior and geometrical properties of packings of elongated particles. Physical Review E, 81:051304:1-17, 2010.

[7] E. Azéma and F. Radjaï. Force chains and contact network topology in sheared packings of elongated particles. Phys. Rev. E, 85(3), 2012.

[8] E. Azéma, F. Radjai, R. Peyroux, and G. Saussine. Force transmission in a packing of pentagonal particles. Physical Review E, 76:011301, 2007.

[9] E. Azéma, F. Radjai, and G. Saussine. Quasistatic rheology, force transmission and fabric properties of a packing of irregular polyhedral particles. Mechanics of Materials, 41(6):729-741, 2009. 
[10] M. Babic, H. H. Shen, and H. T. Shen. The stress tensor in granular shear flows of uniform, deformable disks at high solids concentrations. J. Fluid Mech., 219:81, 1990.

[11] K. Bagi. Microstructural stress tensor of granular assemblies with volume forces. $J$. Appl. Mech., 66:934-936, 1999.

[12] J. P. Bardet. Observations on the effects of particle rotations on the failure of idealized granular materials. Mechanics of Materials, 18:159-182, 1994.

[13] J. P. Bardet and J. Proubet. A numerical investigation of the structure of persistent shear bands in granular media. Géotechnique, 41(4):599-613, 1991.

[14] Y. M. Bashir and J. D. Goddard. A novel simulation method for the quasi-static mechanics of granular assemblages. J. Rheol., 35(5):849-885, 1991.

[15] L. Bécu, S. Manneville, and A. Colin. Yielding and flow in adhesive and nonadhesive concentrated emulsions. Phys. Rev. Lett., 96:138302, 2006.

[16] Y. Bertho, F. Giorgiutti-Dauphine, and J.-P. Hulin. Intermittent dry granular flow in a vertical pipe. Phys. Fluids, 15(11):3358-3369, 2003.

[17] D. Bi, J. Zhang, B. Chakraborty, and R. P. Behringer. Jamming by shear. Nature, 480(7377):355-358, 2011.

[18] D. L. Blair, N. W. Mueggenburg, A. H. Marshall, H. M. Jaeger, and S. R. Nagel. Force distributions in 3d granular assemblies: Effects of packing order and inter-particle friction. Phys. Rev. E, 63:041304-1, 2001.

[19] T. Börzsönyi, T. Unger, and B. Szabó. Shear zone refraction and deflection in layered granular materials. Phys. Rev. E, 80:060302, Dec 2009.

[20] T. Borzsonyi, T. Unger, B. Szabo, S. Wegner, F. Angenstein, and R. Stannarius. Reflection and exclusion of shear zones in inhomogeneous granular materials. Soft Matter, 7:8330-8336, 2011.

[21] L. Brendel, J. Török, R. Kirsch, and U. Bröckel. A contact model for the yielding of caked granular materials. Granular Matter, 13(6):777-786, 2011.

[22] J. Bridgwater. On the width of failure zones. Géotechnique, 30:533, 1980.

[23] N. V. Brilliantov, N. Albers, F. Spahn, and T. PÃPschel. Collision dynamics of granular particles with adhesion. Physical Review E, 76(5), 2007.

[24] N. V. Brilliantov and T. Pöschel. Collision of Adhesive Viscoelastic Particles. Wiley VCH, 2005. 
[25] N. V. Brilliantov, F. Spahn, J. M. Hertzsch, and T. Pöschel. Model for collisions in granular gases. Physical Review E, 53(5):5382-5392, May 1996.

[26] A. Brucks, T. Arndt, J. M. Ottino, and R. M. Lueptow. Behavior of flowing granular materials under variable $g$. Phys. Rev. E, 75:032301, Mar 2007.

[27] J. Brujić, S. F. Edwards, D. V. Grinev, I. Hopkinson, D. Brujić, and H. A. Makse. $3 \mathrm{D}$ bulk measurements of the force distribution in a compressed emulsion system. Faraday Discussions, 123:207-220, Jan. 2003.

[28] C. S. Campbell. Granular shear flows at the elastic limit. Journal of Fluid Mechanics, 465:261-291, 2002.

[29] A. Castellanos. The relationship between attractive interparticle forces and bulk behavior in dry and uncharged fine powders. Advances in Physics, 54(4):263-376, 2005.

[30] P. Chaudhuri, L. Berthier, and L. Bocquet. Inhomogeneous shear flows in soft jammed materials with tunable attractive forces. Phys. Rev. E, 85:021503, 2012.

[31] X. Cheng, J. B. Lechman, A. Fernandez-Barbero, G. S. Grest, H. M. Jaeger, G. S. Karczmar, M. E. Möbius, and S. R. Nagel. Three-dimensional shear in granular flow. Phys. Rev. Lett., 96(3):038001, 2006.

[32] M. P. Ciamarra, R. Pastore, M. Nicodemi, and A. Coniglio. Jamming phase diagram for frictional particles. Phys. Rev. E, 84:041308, Oct. 2011.

[33] P. A. Cundall. A computer model for simulating progressive, large-scale movements in blocky rock systems. In Proc. Symp. Int. Rock Mech., volume 2, Nancy, 1971.

[34] P. A. Cundall and O. D. L. Strack. A discrete numerical model for granular assemblies. Géotechnique, 29(1):47-65, 1979.

[35] F. da Cruz, S. Emam, M. Prochnow, J.-N. Roux, and F. m. c. Chevoir. Rheophysics of dense granular materials: Discrete simulation of plane shear flows. Phys. Rev. E, 72:021309, Aug 2005.

[36] B. Dahneke. Measurements of bouncing of small latex spheres. Journal of Colloid and Interface Science, 45(3):584 - 590, 1973.

[37] P. G. de Gennes. Granular matter: a tentative view. Reviews of Modern Physics, 71(2):374-382, 1999.

[38] M. Depken, J. B. Lechman, M. V. Hecke, W. V. Saarloos, and G. S. Grest. Stresses in smooth flows of dense granular media. EPL (Europhysics Letters), 78(5):58001, 2007.

[39] M. Depken, W. van Saarloos, and M. van Hecke. Continuum approach to wide shear zones in quasistatic granular matter. Phys. Rev. E, 73:031302, 2006. 
[40] B. Derjaguin, V. Muller, and Y. P. Toporov. Effect of contact deformations on the adhesion of particles. Journal of Colloid and interface science, 53(2):314-326, 1975.

[41] J. Desrues and G. Viggiani. Strain localization in sand: an overview of the experimental results obtained in grenoble using stereophotogrammetry. International Journal for Numerical and Analytical Methods in Geomechanics, 28(4):279-321, 2004.

[42] J. K. G. Dhont, M. P. Lettinga, Z. Dogic, T. A. J. Lenstra, H. Wang, S. Rathgeber, P. Carletto, L. Willner, H. Frielinghaus, and P. Lindner. Shear-banding and microstructure of colloids in shear flow. Faraday Discuss., 123:157-172, 2003.

[43] J. A. Dijksman and M. van Hecke. Granular flows in split-bottom geometries. Soft Matter, 6:2901-2907, 2010.

[44] J. A. Dijksman, E. Wandersman, S. Slotterback, C. R. Berardi, W. D. Updegraff, M. van Hecke, and W. Losert. From frictional to viscous behavior: Three-dimensional imaging and rheology of gravitational suspensions. Physical Review E, 82(6):060301, 2010.

[45] J. Duran. Sands, Powders, and Grains-An Introduction to the Physics of Granular Materials. Springer, New York, 2000.

[46] O. Durán, N. P. Kruyt, and S. Luding. Analysis of three-dimensional micromechanical strain formulations for granular materials: Evaluation of accuracy. International Journal of Solids and Structures, 47(2):251-260, 2010.

[47] O. Durán, N. P. Kruyt, and S. Luding. Micro-mechanical analysis of deformation characteristics of three-dimensional granular materials. International Journal of Solids and Structures, 47(17):2234-2245, 2010.

[48] W. Ehlers and W. Volk. Cosserat-Theorie für gesättigte poröse Festkörper. Zeitschrift für Angewandte Mathematik und Mechanik (ZAMM), 77(Supplement 1):83-84, 1997.

[49] J. M. Erikson, N. W. Mueggenburg, H. M. Jaeger, and S. R. Nagel. Force distributions in three-dimensional compressible granular packs. Phys. Rev. E, 66(4), 2002.

[50] N. Estrada, A. Lizcano, and A. Taboada. Simulation of cemented granular materials. i. macroscopic stress-strain response and strain localization. Phys. Rev. E, 82:011303, 2010 .

[51] Y. Fan and K. M. Hill. Shear-driven segregation of dense granular mixtures in a splitbottom cell. Phys. Rev. E, 81:041303, Apr 2010.

[52] D. Fenistein, J. W. van de Meent, and M. van Hecke. Universal and wide shear zones in granular bulk flow. Phys. Rev. Lett., 92:094301, 2004. 
[53] D. Fenistein, J. W. van de Meent, and M. van Hecke. Core precession and Global Modes in Granular Bulk Flow. Phys. Rev. Lett., 96:118001, 2006.

[54] D. Fenistein and M. van Hecke. Kinematics - Wide shear zones in granular bulk flow. Nature, 425(6955):256, 2003.

[55] Y. Forterre and O. Pouliquen. Flows of dense granular media. Annu. Rev. Fluid Mech., 40:1-24, 2008.

[56] F. Gilabert, J.-N. Roux, and A. Castellanos. Computer simulation of model cohesive powders: plastic consolidation, structural changes, and elasticity under isotropic loads. Physical Review E, 78(3):031305, 2008.

[57] I. Goldhirsch. Rapid Granular Flows. Annu. Rev. Fluid Mech., 35:267-293, 2003.

[58] F. Göncü, O. Duran, and S. Luding. Constitutive relations for the isotropic deformation of frictionless packings of polydisperse spheres. C. R. Mécanique, 338(1011):570-586, 2010.

[59] G. Haiat, M. P. Huy, and E. Barthel. The adhesive contact of viscoelastic spheres. Journal of the Mechanics and Physics of Solids, 51(1):69 - 99, 2003.

[60] M. Harrington, J. H. Weijs, and W. Losert. Suppression and emergence of granular segregation under cyclic shear. Phys. Rev. Lett., 111:078001, Aug 2013.

[61] R. R. Hartley and R. P. Behringer. Logarithmic rate dependence of force networks in sheared granular materials. Nature, 421(6926):928-931, 2003.

[62] A. Hatzes, F. Bridges, D. Lin, and S. Sachtjen. Coagulation of particles in saturn's rings: Measurements of the cohesive force of water frost. Icarus, 89(1):113-121, 1991.

[63] A. P. Hatzes, F. Bridges, D. N. C. Lin, and S. Sachtjen. Coagluation of particles in Saturn's Rings: Measurements of the cohesive force of water frost. Icarus, 89:113, 1991.

[64] D. L. Henann and K. Kamrin. A predictive, size-dependent continuum model for dense granular flows. Proceedings of the National Academy of Sciences, 2013.

[65] S. Herminghaus. Dynamics of wet granular matter. Advances in Physics, 54(3):221$261,2005$.

[66] H. J. Herrmann and S. Luding. Modeling granular media with the computer. Continuum Mechanics and Thermodynamics, 10:189-231, 1998.

[67] K. M. Hill and Y. Fan. Isolating segregation mechanisms in a split-bottom cell. Phys. Rev. Lett., 101:088001, Aug 2008. 
[68] R. Höhler and S. Cohen-Addad. Rheology of liquid foam. Journal of Physics: Condensed Matter, 17(41):R1041, 2005.

[69] K. A. Howard. Avalanche mode of motion: Implications from lunar examples. Science, 180(4090):1052-1055, 1973.

[70] D. Howell, R. P. Behringer, and C. Veje. Stress fluctuations in a 2d granular Couette experiment: A continuous transition. Phys. Rev. Lett., 82(26):5241-5244, 1999.

[71] O. I. Imole, N. Kumar, V. Magnanimo, and S. Luding. Hydrostatic and shear behavior of frictionless granular assemblies under different deformation conditions. KONA, 30:84-108, 2013.

[72] H. M. Jaeger, S. R. Nagel, and R. P. Behringer. Granular solids, liquids, and gases. Reviews of Modern Physics, 68(4):1259-1273, 1996.

[73] R. Jasevičius, R. Kačianauskas, and J. Tomas. Simulation of normal impact of micronsized particle with elastic-plastic contact. Journal of Vibroengineering, 11(1):6-16, 2009.

[74] R. Jasevičius, J. Tomas, and R. Kačianauskas. Simulation of normal impact of ultrafine silica particle on substrate. Particulate Science and Technology, 29(2):107-126, 2011.

[75] M. Jiang, Y. Sun, L. Li, and H. Zhu. Contact behavior of idealized granules bonded in two different interparticle distances: An experimental investigation. Mechanics of Materials, 55(0):1 - 15, 2012.

[76] M. Jiang, W. Zhang, Y. Sun, and S. Utili. An investigation on loose cemented granular materials via dem analyses. Granular Matter, 15(1):65-84, 2013.

[77] K. L. Johnson. Contact Mechanics. Cambridge Univ. Press, Cambridge, 1989.

[78] K. K. Johnson K. L and R. A. D. Surface energy and the contact of elastic solids. Proc. R. Soc. Lond. A, 324(1558):301-313, 1971.

[79] P. Jop. Hydrodynamic modeling of granular flows in a modified Couette cell. Phys. Rev. E, 77:032301, 2008.

[80] P. Jop, Y. Forterre, and O. Pouliquen. A constitutive law for dense granular flows. Nature, 441:727-730, 2006.

[81] A. J. Kabla and T. J. Senden. Dilatancy in Slow Granular Flows. Physical Review Letters, 102(22), 2009.

[82] K. Kamrin and G. Koval. Nonlocal Constitutive Relation for Steady Granular Flow. Phys. Rev. Lett., 108:178301, Apr. 2012. 
[83] G. Katgert, M. E. Möbius, and M. van Hecke. Rate dependence and role of disorder in linearly sheared two-dimensional foams. Phys. Rev. Lett., 101:058301, 2008.

[84] L. Kempton, D. Pinson, S. Chew, P. Zulli, and A. Yu. Simulation of macroscopic deformation using a sub-particle $\{$ DEM $\}$ approach. Powder Technology, 223(0):1926, 2012.

[85] S. P. Klein and B. R. White. Dynamic shear of granular material under variable gravity conditions. AIAA Journal, 28:1701-1702, 1990.

[86] S. Kothe, J. Blum, R. Weidling, and C. Güttler. Free collisions in a microgravity manyparticle experiment. iii. the collision behavior of sub-millimeter-sized dust aggregates. Icarus, 225(1):75-85, 2013.

[87] N. Kumar, O. I. Imole, V. Magnanimo, and S. Luding. Evolution of the Effective Moduli for Anisotropic Granular Materials during Shear. In S. Luding and A. Yu, editors, Powders \& Grains 2013, pages 1238-1241, Sydney, Australia, 2013. Balkema.

[88] N. Kumar, V. Magnanimo, and S. Luding. The evolution of anisotropy in sheared granular materials: numerical simulation and theoretical prediction. Acta Geotechnica, Under Review, 2013.

[89] G. Kuwabara and K. Kono. Restitution Coefficient in a Collision between two Spheres. Japanese Journal of Applied Physics, 26(8):1230-1233, 1987.

[90] A. Kwade, D. Schulze, and J. Schwedes. Determination of the stress ratio in uniaxial compression tests - Part 1. Powder handling \& Processing, 6(1):61-65, 1994.

[91] M. Lätzel, S. Luding, and H. J. Herrmann. Macroscopic material properties from quasi-static, microscopic simulations of a two-dimensional shear-cell. Granular Matter, 2(3):123-135, 2000.

[92] M. Lätzel, S. Luding, H. J. Herrmann, D. W. Howell, and R. P. Behringer. Comparing simulation and experiment of a 2D granular Couette shear device. The European Physical Journal E: Soft Matter and Biological Physics, 11(4):325-333, 2003.

[93] J. Li, F. Spaepen, and T. C. Hufnagel. Nanometre-scale defects in shear bands in a metallic glass. Philosophical Magazine A, 82(13):2623-2630, 2002.

[94] X. S. Li and Y. F. Dafalias. Anisotropic Critical State Theory: Role of Fabric. Journal of Engineering Mechanics, 138(3):263-275, Mar. 2012.

[95] K. Liffman, D. Y. C. Chan, and B. D. Hughes. Force distribution in a two dimensional sandpile. Powder Technology, 72(3):255-267, 1992.

[96] A. J. Liu and S. R. Nagel. Nonlinear dynamics: Jamming is not just cool any more. Nature, 396(6706):21-22, 1998. 
[97] C. H. Liu, S. R. Nagel, D. A. Schecter, S. N. Coppersmith, S. Majumdar, O. Narayan, and T. A. Witten. Force fluctuations in bead packs. Science, 269(5223):513-515, 1995.

[98] G. Løvoll, K. J. Måløy, and E. G. Flekkøy. Force measurements on static granular materials. Physical Review E, 60(5):5872, 1999.

[99] S. Luding. Models and Simulations of Granular Materials. PhD thesis, Universität Freiburg, 1994.

[100] S. Luding. Collisions \& Contacts between two particles. In H. J. Herrmann, J. P. Hovi, and S. Luding, editors, Physics of dry granular media - NATO ASI Series E350, page 285, Dordrecht, 1998. Kluwer Academic Publishers.

[101] S. Luding. Particulate Solids Modeling with Discrete Element Methods. In P. Massaci, G. Bonifazi, and S. Serranti, editors, CHoPS-05 CD Proceedings, pages 1-10, Tel Aviv, 2006. ORTRA.

[102] S. Luding. Cohesive, frictional powders: contact models for tension. Granular Matter, 10(4):235-246, 2008.

[103] S. Luding. Constitutive relations for the shear band evolution in granular matter under large strain. Particuology, 6(6):501-505, 2008.

[104] S. Luding. Introduction to discete element methods: Basics of contact force models and how to perform the micro-marco transition to continuum theory. Euro. J. of Enviro. Civ. Eng., 12(7-8):785-826, 2008.

[105] S. Luding. The effect of friction on wide shear bands. Particulate Science and Technology, 26(1), 2008.

[106] S. Luding. From molecular dynamics and particle simulations towards constitutive relations for continuum theory. In B. Koren and K. Vuik, editors, Advanced Computational Methods in Science and Engineering, Lecture Notes in Computational Science and Engineering. Springer, 2009.

[107] S. Luding and F. Alonso-Marroquín. The critical-state yield stress (termination locus) of adhesive powders from a single numerical experiment. Granular Matter, 13(2):109-119, 2011.

[108] S. Luding, E. Clément, A. Blumen, J. Rajchenbach, and J. Duran. Interaction Laws and the Detachment Effect in Granular Media. In Fractal Aspects of Materials, volume 367, pages 495-500, Pittsburgh, Pennsylvania, 1995. Materials Research Society, Symposium Proceedings. 
[109] S. Luding and H. J. Herrmann. Micro-macro transition for cohesive granular media. Zur Beschreibung komplexen Materialverhaltens, Institut für Mechanik, S. Diebels (Ed.), Stuttgart, pages 121-133, 2001.

[110] S. Luding, K. Manetsberger, and J. Mullers. A discrete model for long time sintering. Journal of the Mechanics and Physics of Solids, 53(2):455-491, 2005.

[111] T. S. Majmudar and R. P. Behringer. Contact force measurements and stress-induced anisotropy in granular materials. Nature, 435(7045):1079-1082, 2005.

[112] H. A. Makse, D. L. Johnson, and L. M. Schwartz. Packing of compressible granular materials. Physical review letters, 84(18):4160, 2000.

[113] G. Mandl, L. Jong, and A. Maltha. Shear zones in granular material. Rock mechanics, 9(2-3):95-144, 1977.

[114] R. Mani, D. Kadau, D. Or, and H. J. Herrmann. Fluid depletion in shear bands. Phys. Rev. Lett., 109:248001, 2012.

[115] G. Mason and W. C. Clark. Liquid bridges between spheres. Chemical Engineering Science, 20(10), 1965.

[116] S. McNamara and W. R. Young. Inelastic collapse in two dimensions. Phys. Rev. E, 50(1):R28-R31, 1994.

[117] S. McNamara and W. R. Young. Dynamics of a freely evolving, two-dimensional granular medium. Phys. Rev. E, 53(5):5089-5100, 1996.

[118] G. D. R. MiDi. On dense granular flows. The European Physical Journal E: Soft Matter and Biological Physics, 14(4):341-365, 2004.

[119] P. Mills, D. Loggia, and M. Tixier. Model for a stationary dense granular flow along an inclined wall. EPL (Europhysics Letters), 45(6):733, 1999.

[120] O. Molerus. Theory of yield of cohesive powders. Powder Technology, 12(3):259275, 1975.

[121] O. Molerus. Effect of interparticle cohesive forces on the flow behaviour of powders. Powder Technology, 20(2):161-175, 1978.

[122] A. Moridi, S. Hassani-Gangaraj, and M. Guagliano. A hybrid approach to determine critical and erosion velocities in the cold spray process. Applied Surface Science, 273:617-624, 2013.

[123] D. M. Mueth, G. F. Debregeas, G. S. Karczmar, P. J. Eng, S. R. Nagel, and H. M. Jaeger. Signatures of granular microstructure in dense shear flows. Nature, 406(6794):385-389, 2000. 
[124] D. M. Mueth, H. M. Jaeger, and S. R. Nagel. Force distribution in a granular medium. Physical Review E, 57(3):3164-3169, Mar. 1998.

[125] H. B. Mühlhaus and I. Vardoulakis. The thickness of shear bands in granular materials. Géotechnique, (37):271-283, 1987.

[126] N. Murdoch, B. Rozitis, S. Green, T.-L. Lophem, P. Michel, and W. Losert. Granular shear flow in varying gravitational environments. Granular Matter, 15(2):129-137, 2013.

[127] N. Murdoch, B. Rozitis, K. Nordstrom, S. Green, P. Michel, T.-L. de Lophem, and W. Losert. Granular convection in microgravity. Physical review letters, 110(1):018307, 2013.

[128] S. Nase, W. L. Vargas, A. A. Abatan, and J. J. McCarthy. Discrete characterization tools for cohesive granular material. Powder Technology, 116(2-3), 2001.

[129] R. M. Nedderman and C. Laohakul. The thickness of the shear zone of flowing granular material. Powder Technol., 25:91, 1980.

[130] M. Oda. Initial fabrics and their relations to mechanical properties of granular materials. Soils and Foundation, 1(12):17-36, 1972.

[131] M. Oda, K. Iwashita, and H. Kazama. Micro-structure developed in shear bands of dense granular soils and its computer simulation - mechanism of dilatancy and failure -. In N. A. Fleck and A. C. E. Cocks, editors, IUTAM Symposium on Mechanics of Granular and Porous Materials, pages 353-364. Kluwer Academic Publishers, 1997.

[132] M. Oda and H. Kazama. Microstructure of shear bands and its relation to the mechanism of dilatancy and failure of dense granular soils. Géotechnique, 48(4):465-481, 1998.

[133] C. S. O’Hern, S. A. Langer, A. J. Liu, and S. R. Nagel. Force Distributions near Jamming and Glass Transitions. Physical Review Letters, 86(1):111-114, 2001.

[134] C. S. O’Hern, S. A. Langer, A. J. Liu, and S. R. Nagel. Random packings of frictionless particles. Phys. Rev. Lett., 88(7), 2002.

[135] C. S. O’Hern, L. E. Silbert, A. J. Liu, and S. R. Nagel. Jamming at zero temperature and zero applied stress: The epitome of disorder. Phys. Rev. E, 68, 2003.

[136] T. Ormel, V. Magnanimo, H. H. ter, and S. Luding. Modeling of asphalt and experiments with a discrete particles method. In Conference Proceedings MAIREPAV7 2012, 2012.

[137] M. Otsuki and H. Hayakawa. Critical scaling near jamming transition for frictional granular particles. Phys. Rev. E, 83(5), 2011. 
[138] M. Otsuki, H. Hayakawa, and S. Luding. Behavior of pressure and viscosity at high densities for two-dimensional hard and soft granular materials. Prog. Theor. Phys. Suppl., 184:110-133, 2010.

[139] J. M. Ottino and D. V. Khakhar. Mixing and Segregation of Granular Materials. Ann. Rev. Fluid Mech., 32:55, 2000.

[140] G. Ovarlez, S. Rodts, X. Chateau, and P. Coussot. Phenomenology and physical origin of shear localization and shear banding in complex fluids. Rheologica acta, 48(8):831-844, 2009.

[141] G. Ovarlez, S. Rodts, A. Ragouilliaux, P. Coussot, J. Goyon, and A. Colin. Wide-gap couette flows of dense emulsions: Local concentration measurements, and comparison between macroscopic and local constitutive law measurements through magnetic resonance imaging. Phys. Rev. E, 78:036307, 2008.

[142] M. Pasha, S. Dogbe, C. Hare, A. Hassanpour, and M. Ghadiri, 2013. Under review.

[143] A.-S. Persson and G. Frenning. An experimental evaluation of the accuracy to simulate granule bed compression using the discrete element method. Powder Technology, 219:249 - 256, 2012.

[144] P.-E. Peyneau and J.-N. Roux. Frictionless bead packs have macroscopic friction, but no dilatancy. Phys. Rev. E, 78:011307, July 2008.

[145] M. Pica Ciamarra and A. Coniglio. Jamming at Zero Temperature, Zero Friction, and Finite Applied Shear Stress. Phys. Rev. Lett., 103(23), 2009.

[146] T. Pöschel and T. Schwager. Computational Granular Dynamics. Springer, Berlin, 2005.

[147] O. Pouliquen. On the shape of granular fronts down rough inclined planes. Physics of Fluids (1994-present), 11(7):1956-1958, 1999.

[148] O. Pouliquen and Y. Forterre. A non-local rheology for dense granular flows. Physical and Engineering Sciences, 367(1909):5091-5107, Dec. 2009.

[149] O. Pouliquen, Y. Forterre, and S. L. Dizes. Slow dense granular flows as a self-induced process. Advances in Complex Systems, 04(04):441-450, 2001.

[150] O. Pouliquen and R. Gutfraind. Stress fluctuations and shear zones in quasi-static granular chute flows. Phys. Rev. E, 53(1):552, 1996.

[151] M. A. S. Quintanilla, A. Castellanos, and J. M. Valverde. Interparticle contact forces in fine cohesive powders. theory and experiments. PAMM, 3:206-207, 2003.

[152] F. Radjai, M. Jean, J. J. Moreau, and S. Roux. Force Distribution in Dense TwoDimensional Granular Systems. Phys. Rev. Lett., 77(2):274, 1996. 
[153] F. Radjai, V. Topin, V. Richefeu, C. Voivret, J. Delenne, E. Azéma, and S. El Youssoufi. Force transmission in cohesive granular media. AIP Conference Proceedings, 1227(1):240-259, 2010.

[154] F. Radjai, D. E. Wolf, M. Jean, and J. Moreau. Bimodal Character of Stress Transmission in Granular Packings. Physical Review Letters, 80:61-64, 1998.

[155] A. Ragouilliaux, G. Ovarlez, N. Shahidzadeh-Bonn, B. Herzhaft, T. Palermo, and P. Coussot. Transition from a simple yield-stress fluid to a thixotropic material. Phys. Rev. E, 76:051408, 2007.

[156] O. Reynolds. On the dilatancy of media composed of rigid particles in contact. Philos. Mag. Ser. 5, 50-20:469, 1885.

[157] V. Richefeu, M. S. El Y., and F. Radjaï. Shear Strength of Unsaturated Soils : Experiments, DEM Simulations, and Micromechanical Analysis. In Theoretical and $\mathrm{Nu}$ merical Unsaturated Soil Mechanics, volume 427. Springer Berlin Heidelberg, 2007.

[158] V. Richefeu, M. S. El Youssoufi, and F. Radjaï. Shear strength properties of wet granular materials. Phys. Rev. E, 73:051304, 2006.

[159] A. Ries, D. E. Wolf, and T. Unger. Shear zones in granular media: Three-dimensional contact dynamics simulations. Phys. Rev. E, 76:051301, 2007.

[160] G. H. Risto and H. J. Herrmann. Density patterns in two-dimensional hoppers. Physical Review E, 50:5, 1994.

[161] P. G. Rognon, J.-N. Roux, M. NaaIM, and F. Chevoir. Dense flows of cohesive granular materials. Journal of Fluid Mechanics, 596:21-47, 2008.

[162] K. Saitoh, A. Singh, V. Magnanimo, and S. Luding. The least dissipation principle in wide shear bands. In preparation, 2014.

[163] K. Sakaie, D. Fenistein, T. J. Carroll, M. van Hecke, and P. Umbanhowar. Mr imaging of reynolds dilatancy in the bulk of smooth granular flows. EPL (Europhysics Letters), 84(3):38001, 2008.

[164] K. Sakaie, D. Fenistein, T. J. Carroll, M. van Hecke, and P. Umbanhowar. Mr imaging of reynolds dilatancy in the bulk of smooth granular flows. EPL (Europhysics Letters), 84(3):38001, 2008.

[165] S. Savage. The Mechanics of Rapid Granular Flows, volume 24 of Advances in Applied Mechanics, pages 289-366. Elsevier, 1984.

[166] S. B. Savage. Analyses of slow high-concentration flows of granular materials. J. Fluid Mech., 377:1-26, 1998. 
[167] P. Schall and M. van Hecke. Shear Bands in Matter with Granularity. Annual Review of Fluid Mechanics, 42(1), 2010.

[168] T. Schmidt, F. Gärtner, H. Assadi, and H. Kreye. Development of a generalized parameter window for cold spray deposition. Acta materialia, 54(3):729-742, 2006.

[169] R. Schwarze, A. Gladkyy, F. Uhlig, and S. Luding. Rheology of weakly wetted granular materials: a comparison of experimental and numerical data. Granular Matter, 15(4), 2013.

[170] J. Schwedes. Testers for measuring flow properties of particulate solids. Powder Handling \& Processing, 12(4):337-354, 2000.

[171] J. Schwedes. Review on testers for measuring flow properties of bulk solids. Granular Matter, 5(1):1-43, 2003.

[172] D. R. Scott. Seismicity and stress rotation in a granular model of the brittle crust. Nature, 381:592-595, 1996.

[173] M. R. Shaebani, M. Madadi, S. Luding, and D. E. Wolf. Influence of polydispersity on micromechanics of granular materials. Phys. Rev. E, 85(1), 2012.

[174] L. E. Silbert. Jamming of frictional spheres and random loose packing. Soft Matter, 6:2918-2924, 2010.

[175] L. E. Silbert, A. J. Liu, and S. R. Nagel. Structural signatures of the unjamming transition at zero temperature. Phys. Rev. E, 73(4, Part 1), 2006.

[176] S. E. Silbert, G. S. Grest, and J. W. Landry. Statistics of the Contact Network in Frictional and Frictionless Granular Packings. Physical Review E., 66:061303(6 Part 1), 2002.

[177] A. Singh and S. Luding. Flow behavior at different shear rates for dry powders. In Proceedings of World Congress on Particle Technology WCPT6. Nürnberg Messe $\mathrm{GmbH}, 2010$.

[178] A. Singh, V. Magnanimo, and S. Luding. Contact model for sticking of adhesive mesoscopic particles. Powder Technology, Under Review, 2013.

[179] A. Singh, V. Magnanimo, and S. Luding. Effect of friction and cohesion on anisotropy in quasi-static granular materials under shear. AIP Conference Proceedings, 1542(1):682-685, 2013.

[180] A. Singh, V. Magnanimo, and S. Luding. Effect of friction on the force distribution in sheared granular materials. NUMGE, Conference Proceeding, 2014.

[181] A. Singh, V. Magnanimo, K. Saitoh, and S. Luding. Does gravity have an effect on the slow shear rheology of granular matter? Submitted, 2014. 
[182] A. Singh, V. Magnanimo, K. Saitoh, and S. Luding. Effect of cohesion on shear banding in quasi-static granular material. Phys. Rev. E, Under Review, 2014.

[183] A. Singh, V. Magnanimo, K. Saitoh, and S. Luding. Three dimensional local rheology of dense granular system. In preparation, 2014.

[184] J. H. Snoeijer, M. van Hecke, E. Somfai, and W. van Saarloos. Force and weight distributions in granular media: Effects of contact geometry. Phys. Rev. E, 67, 2003.

[185] C. Song, P. Wang, and H. A. Makse. A phase diagram for jammed matter. Nature, 453(7195), 2008.

[186] C. Sorace, M. Louge, M. Crozier, and V. Law. High apparent adhesion energy in the breakdown of normal restitution for binary impacts of small spheres at low speed. Mechanics Research Communications, 36(3):364 - 368, 2009.

[187] F. Spaepen. A microscopic mechanism for steady state inhomogeneous flow in metallic glasses. Acta Metallurgica, 25(4):407-415, 1977.

[188] L. Staron and F. Radjai. Friction versus texture at the approach of a granular avalanche. Physical Review E, 72(4):041308, 2005.

[189] T. V. Steenkiste, J. Smith, R. Teets, J. Moleski, D. Gorkiewicz, R. Tison, D. Marantz, K. Kowalsky, W. R. II, P. Zajchowski, B. Pilsner, R. McCune, and K. Barnett. Kinetic spray coatings. Surface and Coatings Technology, 111(1):62 - 71, 1999.

[190] A. S. J. Suiker and N. A. Fleck. Frictional collapse of granular assemblies. Journal of Applied Mechanics, 71:350-358, 2004.

[191] H. Tanaka, K. Wada, T. Suyama, and S. Okuzumi. Growth of cosmic dust aggregates and reexamination of particle interaction models. Progress of Theoretical Physics Supplement, 195:101-113, 2012.

[192] G. I. Tardos, S. McNamara, and I. Talu. Slow and intermediate flow of a frictional bulk powder in the Couette geometry. Powder Technology, 131(1):23-39, 2003.

[193] S. C. Thakur, H. Ahmadian, J. Sun, and J. Y. Ooi. An experimental and numerical study of packing, compression, and caking behaviour of detergent powders. Particuology, 2013.

[194] C. Thornton. Numerical simulations of deviatoric shear deformation of granular media. Geotechnique, 50(1):43-53, 2000.

[195] C. Thornton and S. J. Anthony. Quasi static deformation of particulate media. Philosophical Transactions of the Royal Society of London. Series A: Mathematical, Physical and Engineering Sciences, 356(1747):2763-2782, 1998. 
[196] C. Thornton, S. J. Cummins, and P. W. Cleary. An investigation of the comparative behaviour of alternative contact force models during elastic collisions. Powder Technology, 210(3):189 - 197, 2011.

[197] C. Thornton and Z. Ning. A theoretical model for the stick/bounce behaviour of adhesive, elastic-plastic spheres. Powder Technology, 12(5):451+, 1998.

[198] C. Thornton and K. K. Yin. Impact of elastic spheres with and without adhesion. Powder Technol., 65:153, 1991.

[199] B. P. Tighe, J. E. S. Socolar, D. G. Schaeffer, W. G. Mitchener, and M. L. Huber. Force distributions in a triangular lattice of rigid bars. Phys. Rev. E, 72:031306, 2005.

[200] K. To, P.-Y. Lai, and H. K. Pak. Jamming of granular flow in a two-dimensional hopper. Physical review letters, 86(1):71, 2001.

[201] Tomas. Assessment of Mechanical Properties of Cohesive Particulate Solids. Part 1: Particle Contact Constitutive Model. Particulate Science And Technology, 19:95-110, 2001.

[202] J. Tomas. Particle Adhesion Fundamentals and Bulk Powder Consolidation. KONA, 18:157-169, 2000.

[203] J. Tomas. Assessment of mechanical properties of cohesive particulate solids. part 2: Powder flow criteria. Particulate Science and Technology: An International Journal, 19:111-129, 2001.

[204] J. Tomas. Adhesion of ultrafine particles-A micromechanical approach. Chemical Engineering Science, 62(7):1997-2010, 2007.

[205] J. Tomas and S. Kleinschmidt. Improvement of flowability of fine cohesive powders by flow additives. Chemical engineering \& technology, 32(10):1470-1483, 2009.

[206] V. Trappe, V. Prasad, L. Cipelletti, P. N. Segre, and D. A. Weitz. Jamming phase diagram for attractive particles. Nature, 411(6839):772-775, 2001.

[207] T. Unger. Refraction of shear zones in granular materials. Phys. Rev. Lett., 98:018301, 2007.

[208] T. Unger. Collective rheology in quasi static shear flow of granular media, Sept. 2010.

[209] T. Unger, J. Török, J. Kertész, and D. E. Wolf. Shear band formation in granular media as a variational problem. Phys. Rev. Lett., 92:214301, May 2004.

[210] J. Valverde, M. Quintanilla, and A. Castellanos. Jamming Threshold of Dry Fine Powders. Phys. Rev. Lett., 92(25), 2004. 
[211] S. van Baars. Discrete Element Analysis of Granular Materials. PhD thesis, Technische Universiteit Delft, Delft, Nederlands, 1996.

[212] A. R. van Eerd, W. G. Ellenbroek, M. van Hecke, J. H. Snoeijer, and T. J. Vlugt. Tail of the contact force distribution in static granular materials. Physical Review E, 75(6):060302, 2007.

[213] M. van Hecke. Jamming of soft particles: geometry, mechanics, scaling and isostaticity. Journal of Physics: Condensed Matter, 22:033101(3), 2009.

[214] C. T. Veje, D. W. Howell, and R. P. Behringer. Kinematics of a 2D granular Couette experiment at the transition to shearing. Phys. Rev. E, 59(1):739-745, 1999.

[215] P. A. Vermeer, S. Diebels, W. Ehlers, H. J. Herrmann, S. Luding, and E. Ramm, editors. Continuous and Discontinuous Modelling of Cohesive Frictional Materials, Berlin, 2001. Springer.

[216] L. Vu-Quoc and X. Zhang. An elastic contact force-displacement model in the normal direction: displacement-driven version. Proc. R. Soc. Lond. A, 455:4013-4044, 1999.

[217] H.-C. W. Wall S., Walter J. and G. L. S. Measurements of kinetic energy loss for particles impacting surfaces. Aerosol Science and Technology, 12(4):926 - 946, 1990.

[218] O. Walton. Potential discrete element simulation applications ranging from airborne fines to pellet beds. In International Conference On Environmental Systems, volume 01, page 2329, 2004.

[219] O. Walton. Review of adhesion fundamentals for micron-scale particles. KONA Powder and Particle, 26:129-141, 2008.

[220] O. R. Walton. Numerical simulation of inelastic, frictional particle-particle interactions. In M. C. Roco, editor, Particulate two-phase flow, page 884, Boston, 1993. Butterworth-Heinemann.

[221] O. R. Walton and R. L. Braun. Stress Calculations for Assemblies of Inelastic Spheres in Uniform Shear. Acta Mechanica, 63:73, 1986.

[222] O. R. Walton and R. L. Braun. Viscosity, Granular-Temperature, and Stress Calculations for Shearing Assemblies of Inelastic, Frictional Disks. J. Rheol., 30(5):949-980, 1986.

[223] O. R. Walton and S. M. Johnson. Simulating the effects of interparticle cohesion in micron-scale powders. In Powders and Grains 2009: Proceedings of the 6th International Conference on Micromechanics of Granular Media, volume 1145, pages 897-900. AIP Publishing, 2009. 
[224] X. Wang, H. P. Zhu, S. Luding, and A. B. Yu. Regime transitions of granular flow in a shear cell: A micromechanical study. Phys. Rev. E, 88:032203, 2013.

[225] G. Webster. Rover team tests mars moves on earth. http://marsrovers.jpl.nasa.gov/newsroom/pressreleases/20050506a.html.

[226] T. Weinhart, R. Hartkamp, A. R. Thornton, and S. Luding. Coarse-grained local and objective continuum description of three-dimensional granular flows down an inclined surface. Physics of Fluids, 25:070605(7), 2013.

[227] D. M. Wood. Soil behaviour and critical state soil mechanics. Cambridge university press, 1990.

[228] J. Wu, H. Fang, S. Yoon, H. Kim, and C. Lee. The rebound phenomenon in kinetic spraying deposition. Scripta Materialia, 54(4):665 - 669, 2006.

[229] Z. Xianglin, W. Xiangkun, W. Jianguo, and J. Zhang. Numerical investigation of the rebounding and the deposition behavior of particles during cold spraying. Acta Metallurgica Sinica(English letters), 24(1):43, 2011.

[230] R. Y. Yang, R. P. Zou, A. B. Yu, and S. K. Choi. Characterization of interparticle forces in the packing of cohesive fine particles. Phys. Rev. E, 78:031302, 2008.

[231] J. Yuan, Q. Zhang, B. Li, and X. Zhao. Experimental analysis of shear band formation in plane strain tests on shanghai silty clay. Bulletin of Engineering Geology and the Environment, 72:107-114, 2013.

[232] D. Zhang, P. Shipway, and D. McCartney. Cold gas dynamic spraying of aluminum: The role of substrate characteristics in deposit formation. Journal of Thermal Spray Technology, 14:109-116, 2005.

[233] H. P. Zhang and H. A. Makse. Jamming transition in emulsions and granular materials. Phys. Rev. E, 72:011301, July 2005.

[234] J. Zhang, T. S. Majmudar, M. Sperl, and R. P. Behringer. Jamming for a 2D granular material. Soft Matter, 6(13):2982-2991, 2010.

[235] X. Zhang and L. Vu-Quoc. Modeling the dependence of the coefficient of restitution on the impact velocity in elasto-plastic collisions. International Journal of Impact Engineering, 27(3):317-341, 2002.

[236] J. Zhao and N. Guo. Unique critical state characteristics in granular media considering fabric anisotropy. Géotechnique, 63(8):695-704, 2013. 


\section{Curriculum vitae}

\section{Abhinendra Singh}

August 2009

May - July 2006

May - July 2007

August 2009 - Present
Integrated Masters of Science in Physics, IIT Kharagpur, India

Internship, School of Physical Sciences, JNU, India

Internship, JNCASR, Bangalore, India

$\mathrm{PhD}$ candidate, University of Twente

\section{Publications}

Journal Papers

1. A. Singh, V. Magnanimo, and S. Luding. Contact model for sticking of adhesive mesoscopic particles. Powder Technology, Under Review, 2013

2. A. Singh, V. Magnanimo, K. Saitoh, and S. Luding. Effect of cohesion on shear banding in quasi-static granular material. Phys. Rev. E, Under Review, 2014

3. A. Singh, V. Magnanimo, K. Saitoh, and S. Luding. Does gravity have an effect on the slow shear rheology of granular matter? Submitted, 2014

4. A. Singh, V. Magnanimo, K. Saitoh, and S. Luding. Three dimensional local rheology of dense granular system. In preparation, 2014

5. K. Saitoh, A. Singh, V. Magnanimo, and S. Luding. The least dissipation principle in wide shear bands. In preparation, 2014

Proceedings

6. A. Singh and S. Luding. Flow behavior at different shear rates for dry powders. In Proceedings of World Congress on Particle Technology WCPT6. Nürnberg Messe GmbH, 2010

7. A. Singh, V. Magnanimo, and S. Luding. Effect of friction and cohesion on anisotropy in quasi-static granular materials under shear. AIP Conference Proceedings, 1542(1):682685,2013 
8. A. Singh, V. Magnanimo, and S. Luding. Effect of friction on the force distribution in sheared granular materials. NUMGE, Conference Proceeding, 2014

Conferences (Talks)

9. A. Singh, and S. Luding, Flow behavior with random driving in sheared cohesivefrictional powder, Jamming and Rheology Conference, April 2010, Cargese, Corsica

10. A. Singh, and S. Luding, Flow behavior at different shear rates for dry powders, World Congress of Particle Technology 6, April 2010, Nuremberg, Germany

11. A. Singh, V. Magnanimo, and S. Luding, Microstructure analysis in shear bands for cohesive-frictional powders, Heterogeneity and Disorder in Physics, 2010, Universidad de Chile, Santiago, Chile

12. A. Singh, A. Thornton, and S. Luding, Stability Analysis of fully coupled equations in granular avalanches ICMM2 2011, September 2011, Paris, France

13. A. Singh, V. Magnanimo, and S. Luding, Cohesive Frictional Powders, From Micro to Macro, Tsinghua University, June 2012, Beijing, China

14. A. Singh, T. Weinhart, V. Magnanimo, and S. Luding, From particles to continuum theory: Shear bands, jamming and dilatancy, 5th Asian Particle Technology, Symposium (APT 2012), June 2012, Singapore

15. A. Singh, T. Weinhart, V. Magnanimo, and S. Luding, From Particles Towards Continuum Theory: Dilatancy and Anisotropy, 8th European Solid Mechanics Conference 2012, July 2012, Graz, Austria

16. A. Singh, V. Magnanimo, and S. Luding, Three dimensional rheology of dense granular system, Burgersdag 2013, January 2013, Enschede, The Netherlands

17. A. Singh, V. Magnanimo, and S. Luding, Effect of cohesion on shear band in dense granular medium, Particles 2013, September 2013, Stuttgart, Germany

18. A. Singh, V. Magnanimo, and S. Luding, Effect of cohesion and friction on the shear banding, Traffic and Granular Flow'13, Jülich, September 2013, Germany

19. A. Singh, V. Magnanimo, and S. Luding, How does a granular material fail on the moon?,Physics@FOM, January 2014, Veldhoven, The Netherlands

Other services to the scientific community

1. Co-Chair: A session on "Mechanics of Granular Media" at "8th European Solid Mechanics Conference 2012".

2. Reviewer: Granular Matter, Particuology, Intl. Journal of Solids and Structuress 